University of Louisville

ThinkIR: The University of Louisville's Institutional Repository

$8-2020$

\title{
Moral distress and moral residue among nurses working in the surgical intensive care unit: a descriptive qualitative approach.
}

Adam Tyler Booth

University of Louisville

Follow this and additional works at: https://ir.library.louisville.edu/etd

Part of the Critical Care Nursing Commons

\section{Recommended Citation}

Booth, Adam Tyler, "Moral distress and moral residue among nurses working in the surgical intensive care unit: a descriptive qualitative approach." (2020). Electronic Theses and Dissertations. Paper 3490.

https://doi.org/10.18297/etd/3490

This Doctoral Dissertation is brought to you for free and open access by ThinkIR: The University of Louisville's Institutional Repository. It has been accepted for inclusion in Electronic Theses and Dissertations by an authorized administrator of ThinkIR: The University of Louisville's Institutional Repository. This title appears here courtesy of the author, who has retained all other copyrights. For more information, please contact thinkir@louisville.edu. 


\title{
MORAL DISTRESS AND MORAL RESIDUE AMONG NURSES WORKING IN THE SURGICAL INTENSIVE CARE UNIT: \\ A DESCRIPTIVE QUALITATIVE APPROACH
}

\author{
By \\ Adam Tyler Booth \\ BSN, RN, Indiana University Southeast, 2010
}

\begin{abstract}
A Dissertation
Submitted to the Faculty of the

School of Nursing of the University of Louisville in Partial Fulfillment of the Requirements

for the Degree of
\end{abstract}

Doctor of Philosophy

in Nursing

School of Nursing

University of Louisville

Louisville, Kentucky

August 2020 



\title{
MORAL DISTRESS AND MORAL RESIDUE AMONG NURSES WORKING IN THE SURGICAL INTENSIVE CARE UNIT: \\ A DESCRIPTIVE QUALITATIVE APPROACH \\ By
}

\author{
Adam T. Booth \\ A Dissertation Approved on
}

June 30, 2020

By the following Dissertation Committee

Dissertation Chair - Becky Christian

Committee member - Celeste Shawler

Committee member - Paul Clark

Committee member - Avery Kolers 


\section{ACKNOWLEDGMENTS}

I thank want to extend the warmest thanks to my close friends, family, and wife, Chelsea Dresner. I also would like to thank my dissertation committee chair, Dr. Becky Christian, and my other doctoral committee members: Drs. Paul Clark, Ann Hamric, Avery Kolers, and Celeste Shawler. I would also like to dedicate the work within this dissertation to Dr. Ann Hamric and carry on the legacy of her work in the field of moral distress. Without this committee's guidance and unwavering support none of this would have been possible. Thanks so much! 


\section{ABSTRACT \\ MORAL DISTRESS AND MORAL RESIDUE AMONG NURSES WORKING IN THE SURGICAL INTENSIVE CARE UNIT: \\ A DESCRIPTIVE QUALITATIVE APPROACH}

Adam T. Booth

June 30, 2020

Introduction: Approximately one in five Americans will die in the intensive care unit (ICU). Nurses spend more time with chronically ill and end-of-life patients than any other healthcare professional, and may perceive some actions in the ICU as immoral. Moral distress is defined as knowing the right course of action to take but being hindered by institutional constraints. Development of the Moral Distress Scale-Revised revealed that $31 \%$ of ICU nurses left a prior position or considered leaving their current nursing job because of moral distress. The purpose of this descriptive qualitative study is to explore surgical ICU (SICU) nurses' perceptions and experiences of moral distress and moral residue, coping, and perceived quality of care.

Methods: A descriptive qualitative approach used in-depth, semi-structured individual interviews with SICU nurses. Purposive sampling methods of maximum likelihood variation and snowball sampling recruited a heterogeneous sample of 21 SICU nurses from two SICUs in a university hospital until theoretical saturation occurred. Essential themes were identified through reflective writing, rewriting, memoing, and content and thematic analysis. Verbatim transcription of interviews and an audit trail ensured rigor 
and trustworthiness of the data in order to highlight descriptions, patterns, uniqueness, and interpretations of firsthand accounts.

Conclusion: Moral issues were highlighted to explore root causes of moral distress at the individual-level for SICU nurses with a deeper understanding of moral distress and moral residue, particularly how nurses cope with the stress involved and their perceptions of the influence of moral distress on quality patient care. Understanding nurses' experiences is essential to addressing ethical issues of moral distress and moral residue before they negatively impact patient outcomes.

Key words: moral distress, moral residue, crescendo effect, nursing, coping, quality of care, intensive care nurses 
TABLE OF CONTENTS

PAGE

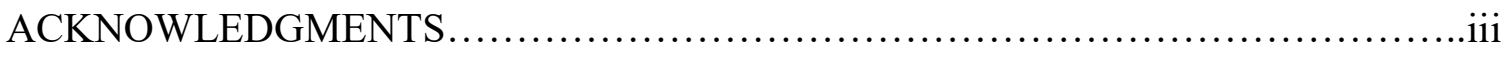

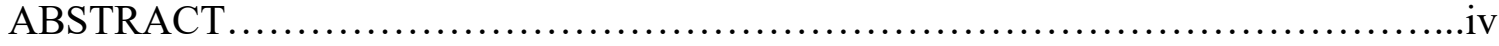

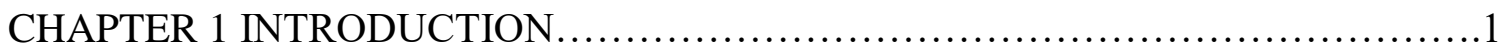

Theoretical Framework.................................................... 18

Crescendo Effect................................................................20

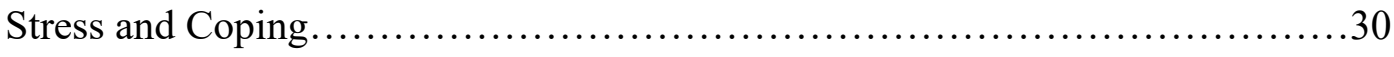

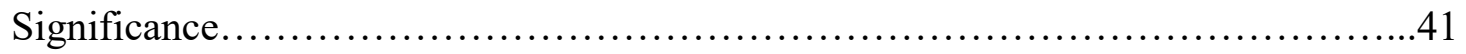

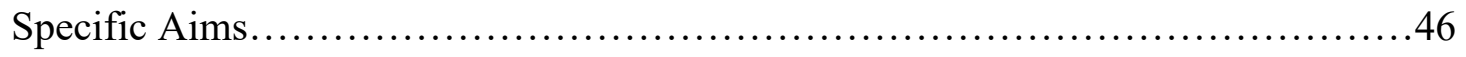

CHAPTER 2 REVIEW OF LITERATURE.......................................47

Background - Moral Distress..............................................47

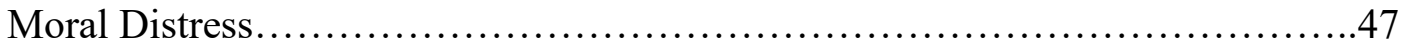

Conceptual Ambiguity of Moral Distress...................................56

Healthcare Rationing, Quality of Life, Effectiveness of Therapies, and Cost......64

Hospital Organizational Ethical Climate and the Ethical Dimensions of Practice..71

Perceptions of Quality Patient Care and Moral Distress.........................76

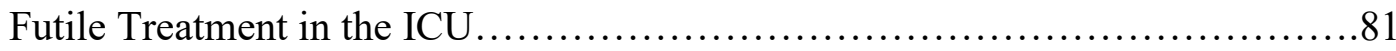

Moral Distress at the End-of-Life............................................ 86

Moral Distress and Conscientious Objection..................................8

Moral Distress and Burnout...............................................95 
Summary......................................................... 100

CHAPTER 3 METHODS....................................................... 109

Design............................................................. 109

Sample............................................................. 111

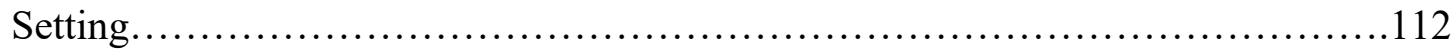

Measures.................................................................... 113

Procedures......................................................................... 113

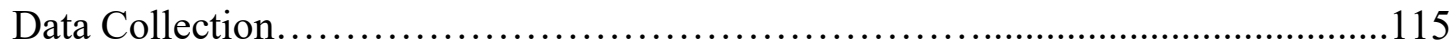

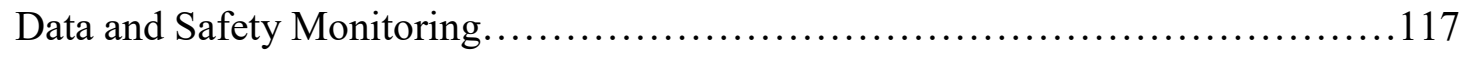

Rigor and Trustworthiness of Data.................................... 117

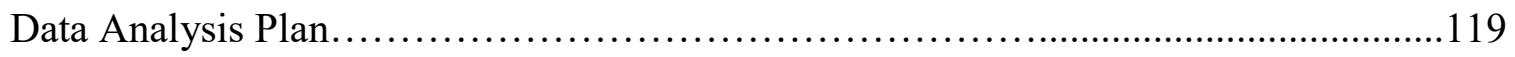

Protection of Human Subjects............................................. 120

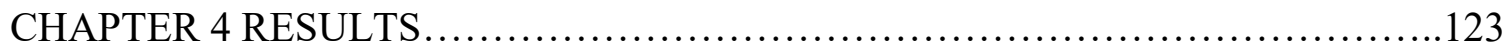

Participants........................................................ 123

Overview of Moral Distress by Nurses in the SICU.........................123

Theme 1 - Concentrated Caring in High Stress Environment-“Doing Your

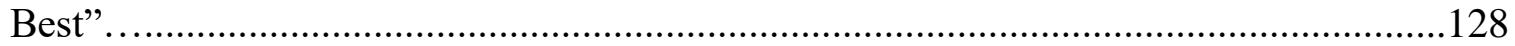

1.a. Doing Your Best for the Patient.................................... 130

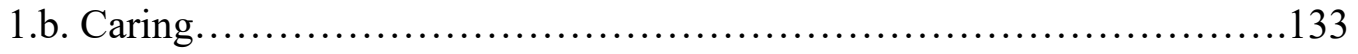

1.c. Communicating with Families of Patients Near the End-of-Life..........136

1.d. Suffering and Inner Turmoil....................................141 
Theme 2 - Patient Advocacy \& Positivity About Nursing - "Doing the Right Thing"

2.a. Physician-Nurse Power Differential................................ 147

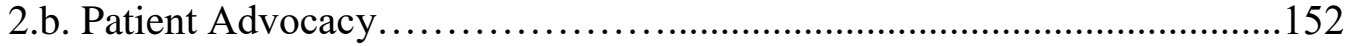

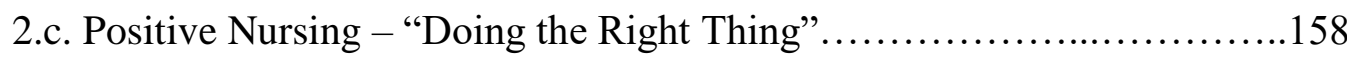

Theme 3 - Surviving Moral Distress in the SICU Environment-Magnifying Stress $\&$ Self-Preservation.

3.a. Nursing Management Problems, Short Staffing, and Lack of Resources..165

3.b. Incompetent RNs Lacking in SICU Experience.

3.c. Demands of Additional Charting and Responsibilities..................176

Theme 4 - The Stain of Moral Residue-Surviving \& Coping with the Impact on Integrity \& Values 186

4.a. Surviving and Coping with the Impact on Personal Moral Integrity and

Values 188

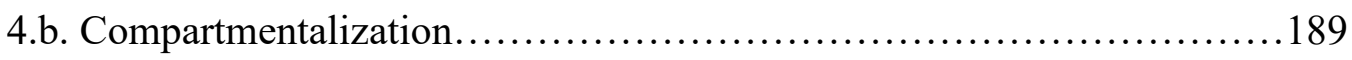

4.c. Nurses "just punching clock" ........................................201

Theme 5 - Moral Crescendo's Coup De Grâce - Suffering and Wounded-

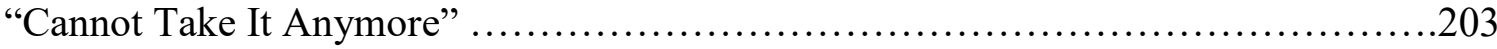

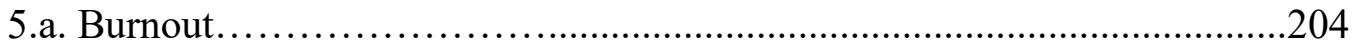

5.b. Cannot Take it Anymore - Ready to Leave...................................206

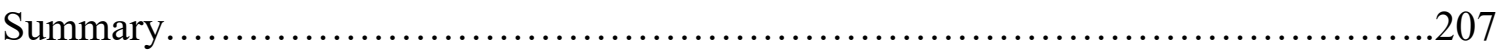

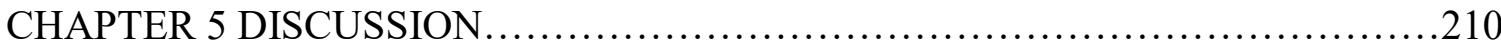

A Practical Definition of Moral Distress_-Violations to Core Values \& Duties. ....211 
Identified Root Causes/Sources of Moral Distress and Moral Residue

Process of Moral Distress, Moral Residue, and the Crescendo Effect.....................231

Coping with the Stress of Moral Distress and Moral Residue......................242

Exploring Moral Distress, Moral Residue, and the Crescendo Effect...............264

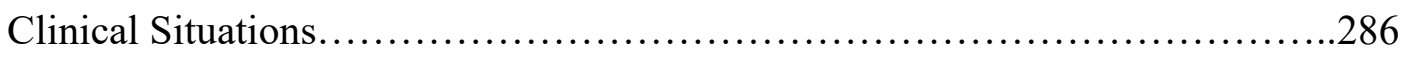

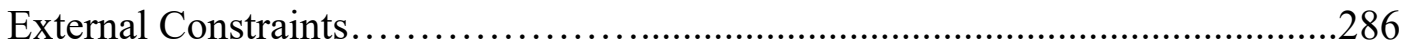

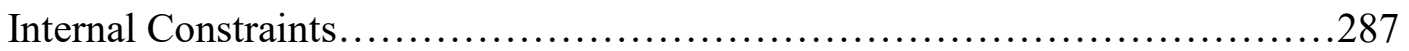

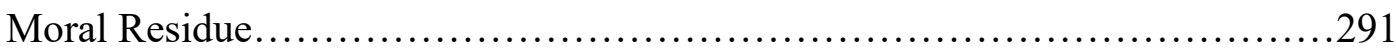

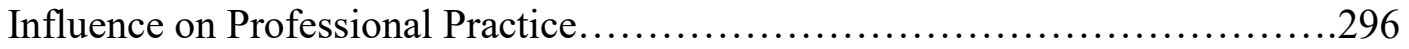

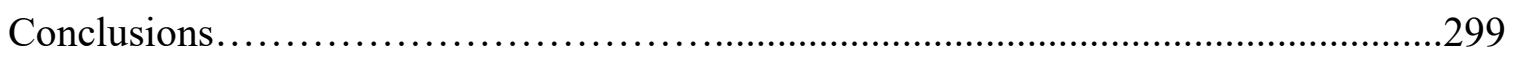

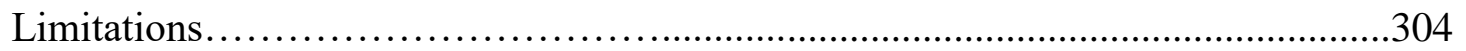

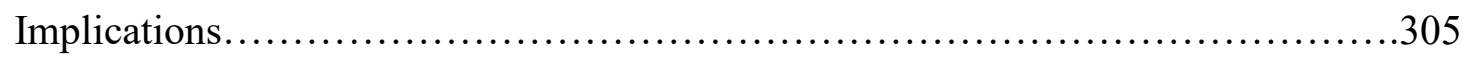

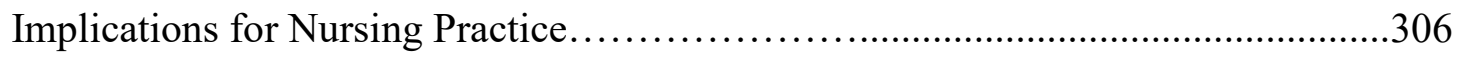

Implications for Nursing Policy ............................................307

Implications for Nursing Education........................................ 308

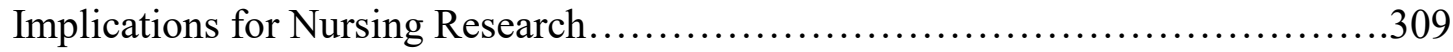

Implications of Ignoring Moral Distress....................................... 311

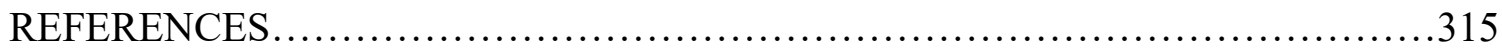

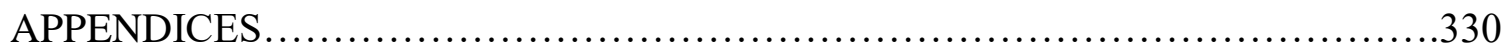

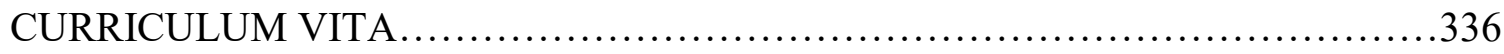




\section{CHAPTER 1 INTRODUCTION}

Approximately one in five Americans will die in the intensive care unit (ICU) (Angus et al., 2004). According to the Dartmouth Atlas of Healthcare (2018), the 2014 national average of deaths associated with ICU admission was 14.7\%. Furthermore, in excess of 90 million Americans live with at least one chronic disease and seven out of 10 die from chronic disease (Dartmouth Atlas of Healthcare, 2018). Additionally, among Medicare recipients, nine of 10 deaths are associated with nine chronic illnesses: congestive heart failure, chronic lung disease, cancer, coronary artery disease, renal failure, peripheral vascular disease, diabetes, chronic liver disease, and dementia (Dartmouth Atlas of Healthcare, 2018). Patients who suffer from chronic illnesses account for about $32 \%$ of total Medicare spending in the form of physician and hospitalization fees from multiple hospitalizations during the last two years of life (Dartmouth Atlas of Healthcare, 2018). The ICU is a complex work environment where multiple healthcare professionals collaborate, communicate, and educate across multiple disciplines to provide specialized technical care to chronically and acutely ill patients (Johnson-Coyle et al., 2016). It is within the ICU environment that the margin for error is small and the consequences of technical medical treatments are potentially life-altering. Within this high-stakes context, moral issues arise and healthcare professionals' ethical evaluations must keep up with the delivery of healthcare in order to promote patients' best interests. 
It is necessary to clarify definitions of morality and integrity to avoid any misrepresentations. Denoting what is moral in a universal way will make that which is considered immoral more easily discernable. The same is true for integrity. If a definition of integrity is also provided, then behaviors or situations that lack integrity will be clear, particularly in a healthcare context.

According to Beauchamp and Childress (2009), morality as a social institution includes standards of conduct, including moral principles (i.e., beneficence, autonomy, justice, non-malfeasance), rules, ideals, rights, and virtues. Morality as a broad concept is more generalized than the common morality. Thus, for the purposes of this dissertation, common morality will endorse views of what is considered a definition of morality, or also what is considered to be a moral course of action in a healthcare context. Common morality is more focused and refers to norms of right and wrong human conduct that are so universal they form a stable social compact (Beauchamp \& Childress, 2009). Individuals claiming to live a moral life must be certain to adhere to several moral rules, which include: do not lie, do not steal others' property, keep promises, respect others' rights, do not kill or cause harm to others (Beauchamp \& Childress, 2009). This set of universal norms all people share is the common morality, particularly because it upholds the general broader concept of that which is moral. Regardless of one's religious, political, or cultural beliefs and practices, the common morality applies to everyone in all places, and human conduct is judged by these standards. According to Beauchamp and Childress (2009), the following is a list of norms that serves as examples of generally binding standards of action (or rules of obligation) found in the common morality that this study endorsed when particular actions were referred to as moral: 
(1) Do not kill, (2) Do not cause pain or suffering to others, (3) Prevent evil or harm from occurring, (4) Rescue persons in danger, (5) Tell the truth, (6) Nurture the young and dependent, (7) Keep your promises, (8) Do not steal, (9) Do not punish the innocent, and (10) Obey just laws. (p. 3)

It is also necessary to tease out a definition of moral integrity, because all individuals, consciously default to a common morality because it is understood as an ethical norm of society that murder and killing is immoral, but integrity is integration of that common morality into one's life. According to Beauchamp and Childress (2009), moral integrity is defined as soundness, reliability, wholeness, and integration of moral character. Integrity is the act of living out the common morality. Specifically, moral integrity is objectivity, impartiality, and fidelity in compliance with moral norms (Beauchamp \& Childress, 2009). The opposite of integrity is a life not in accordance with moral norms, or the common morality. The virtue of integrity illuminates two aspects of an individual's character: (1) the coherent integration of aspects of the self, such as emotions, aspirations, and knowledge (each complements and does not confuse the others); and (2) the character trait of being faithful to moral values and standing up in their defense when necessary (Beauchamp \& Childress, 2009). In summary, the common morality, or what this dissertation will refer to as morality, are generally binding standards of action that all individuals must follow in their everyday lives. Integrity is not only the integration of these moral values into one's life and standing up for them when challenged, but one's character is embedded with moral rules and obligations to the point that they permeate one's emotions, intellect, and goals and ambitions. 
Nurses spend more time with patients than any other healthcare professional (Robinson, 2010). Specifically, a study of moral distress and burnout among healthcare professionals in the cardiovascular surgical ICU found that nurses and nurse practitioners with two to five years of experience had greater moral distress than those with less than two years of experience, and their perceptions of moral distress were affected more by the level of disturbance or intensity, rather than the frequency of occurrence (JohnsonCoyle et al., 2016). Hamric and Blackhall (2007) found that total moral distress scores on an adapted version of the Moral Distress Scale (MDS) were driven primarily by nurses' perceptions of the frequency of morally distressing situations. For example, even though nurses and physicians were both distressed by the provision of futile treatment, nurses perceived that it happened more often than physicians. According to Hamric and Blackhall (2007), the reason for this difference may be the result of physicians being able to move to other units; however, nurses cannot leave stressful situations and are obligated to stay on the unit with their patients and this may influence their perceptions of the frequency of distressing situations. Johnson-Coyle et al. (2016) found that cardiovascular surgical ICU nurses experienced higher levels of moral distress when they had more experience and their perceptions of moral distress were impacted more by the level of disturbance, rather than the frequency. Disparate perspectives remain in the literature regarding frequency and intensity of morally distressing experiences. As a result, it is important to explore nurses' coping abilities because it may influence nurses' perceptions of the frequency and/or intensity of morally distressing events and subsequent perceptions of quality patient care. 
By nature of their contact with patients in a high stress environment where disturbing critical events occur, nurses may perceive some actions in the ICU as immoral and this may lead to moral distress because of the intensity of the disturbance (Epstein, Hurst, Mahanes, Marshall, \& Hamric, 2016). For example, nurses may be unable to dissuade a family from continuing aggressive treatment of a patient near the end-of-life, and thus, continue to carry out their treatment directives even though the nurses feel like they are torturing the patient. Furthermore, ICU nurses may feel that the physician in the ICU is not providing adequate pain medicine for a patient for fear of overmedicating or perhaps hastening death. In this example, nurses are forced to provide sub-optimal care where the patient only receives minimal relief. Nurses may know the right moral action to take: to advocate for the patient's best interests, but prevented by the family in the former and by the physician in the latter example.

Numerous sources of moral distress or root causes exist. In particular, nurses may experience moral distress associated with following the family's wishes to continue treatment that is not in the patient's best interests, communicating poorly, lacking continuity of care, staffing inadequacies, working with incompetent providers, and providing sub-optimal care because of pressure to reduce costs by administrators (Epstein \& Hurst, 2017). Medical futility is one of the many root causes of moral distress (Hamric et al., 2012). Other root causes include: wasting resources, working with incompetent healthcare professionals, following the family's wishes for patient care for fear of a lawsuit, and the provision of false hope to patients and families (Epstein \& Hamric, 2009; Hamric et al., 2012). Moral distress is an alarm bell stemming from being compelled or pressured to act or not act in response to a variety of constraints (Weber, 2016). In 
particular, nurses' experiences of moral distress are potentially symptomatic of moral issues in healthcare such as futile treatment at the end-of-life. Specifically, the experience of moral distress represents incoherence between one's beliefs and actions because one holds a sincere belief about what is right, but one's action or inaction is opposite (Webster \& Baylis, 2000). Similarly, moral distress is a violation of one's core values and duties as a nurse, thus the moral component that makes this concept unique (Epstein \& Hamric, 2009). This hallmark trait of moral distress is that the nurse knows the right course of action to choose, but is unable to take it. The experience of moral distress not only creates powerlessness, but also an overwhelming sense of futility because nurses are not able to create change for the patient's positive benefit-whether that be focusing on palliative care at the end-of-life rather than aggressive treatments, or promoting transparent communication and collaboration between the various healthcare professionals, nurses, patient, and family members.

Moral distress is not just an emotional response or mental state of a nurse; it is a divergence between knowledge and capacity to act on that knowledge. For example, the nurse experiences powerlessness, conflicting loyalties, and a generalized inability to effectuate change for the patient's benefit; thus the nurse feels negatively about their experience of moral distress. However, if a moral issue is present, regardless of the nurse's emotional response, it is still necessary for the nurse to evaluate that issue in order to promote patient safety and prevent poor quality of care. A consequence of moral distress is a sense of powerlessness to act because the nurse's moral integrity has been compromised. 
Andrew Jameton (1984) initially defined moral distress as knowing the right course of action to take, but being hindered because of institutional constraints. This definition focuses on the unethical action, because the nurse is prevented from doing what they believe to be the moral action. Moreover, Webster and Baylis (2000) aptly defined moral distress when they wrote a chapter in a book on medical error-and their definition highlights the nuances within the phenomenon — as arising when one fails to do what they believe to be the right action (or fails to do so to one's satisfaction) for one or more of the following explanations: a judgment error, a personal failing (e.g., a character weakness such as systemic avoidance), or some circumstance beyond one's control. According to Epstein et al. (2016), the definition of moral distress identifies that nurses take moral responsibility when patients are not receiving care that is appropriate, effective, or safe, even when forces outside their control prevent remedying the situation (e.g., a physician's orders, institutional hierarchy such as a policy or a management decision, and/or a family's treatment directives for their incapacitated patient's treatment plan). Thus, the central theme to the definition of moral distress that must be acknowledged is it requires only that the current action under investigation be perceived as morally wrong - a core violation of one's values and duties as a nurse caring for vulnerable and sick patients in need (Epstein \& Hamric, 2009). A definition of moral distress does not require the nurse to be certain of the right action. Also, it does not require moral residue or that the situation be life altering. Rather, the nurse perceives an unethical course of action.

Moral distress leads to compromised integrity and moral residue. Moral residue occurs when a nurse's moral distress is unaddressed and the moral and emotional 
baggage remains (Epstein \& Hamric, 2009). According to Webster and Baylis (2000), moral residue is defined as, "that which each of us carries with us from those times in our lives when in the face of moral distress, we have seriously compromised ourselves or allowed ourselves to be compromised" (p. 218). Moral residue is the lingering experience created from numerous unresolved morally-distressing events, which accumulate, pile up, and lead to further distress (Epstein \& Hamric, 2009). This experience is painful because it threatens and betrays deeply held and cherished beliefs and values, thus the experience of moral residue is lasting and powerfully concentrated in one's thoughts (Webster \& Baylis, 2000).

Based on Epstein's dissertation research (Epstein, 2007), Epstein and Hamric (2009) hypothesized that moral residue can eventually crescendo and become unmanageable and may manifest professionally as conscientious objection, emotional and psychological distancing from the ethical dimensions of practice, burnout, and/or withdrawal from the nursing profession. It remains unclear how the crescendo effect evolves over time and with the intensity and frequency of morally distressing root causes. Nurses may cope with moral distress by using problem- and emotion-focused coping (Lazarus \& Folkman, 1984). Because no two people cope with distress in the same way, it is important to explore ICU nurses' coping abilities with moral distress because there are constructive (e.g., journaling, exercise, communicating with a work peer) and maladaptive (e.g., excessive alcohol consumption, smoking cigarettes, illicit drug usage) ways to reconcile one's experience of powerlessness associated with moral distress.

Epstein and Hamric's (2009) Crescendo Effect (see Figure 1 in Appendix A) and Lazarus and Folkman's (1984) Transactional Model of Stress and Coping are two 
theoretical frameworks that informed this dissertation study. These theoretical frameworks guided the study because moral distress is an experience characterized by keen moral responsibility, concern for the patient's well-being, and is accompanied by a perception of powerlessness (Dudzinski, 2016; Hamric et al., 2012) and depends on temporal, contextual, and situational specific factors (Epstein \& Hamric, 2009; Hamric, 2014). The results of this dissertation demonstrated ethical dimensions of nursing practice create stress in nurses and influence how nurses respond and cope with moral distress, moral residue, and perceptions of the quality of patient care as they provide care and develop emotional interrelationships with patients, families, and other members of the interdisciplinary healthcare team.

The experience of moral residue can be the result of error or the cause of error (Webster \& Baylis, 2000). For example, moral residue results from the realization of error when the nurse recognizes the selection of a particular course of action for reasons of expediency, laziness, or cowardice; or the nurse may simply have the perception of error as when, irrespective of any objective evaluation, the nurse believes they have done something wrong (Webster \& Baylis, 2000). When moral residue is the cause of error, the critical factor is the incremental loss of commitment to previously held values for reasons of self-interest, such as self-protection or self-promotion (Webster \& Baylis, 2000). The nurse in this case may have experienced a high frequency of morally distressing situations, a particularly disturbing and intense event, or some combination of frequent and disturbing experience(s), but the outcome remains the same. Specifically, moral residue is the cause of error when nurses feel powerless and disenfranchised due to 
repeated challenges to their moral integrity as the distress piles up and desensitizes their ability to handle moral issues.

Moral residue occurs typically when individuals recognize that their moral integrity has been compromised and an outward appearance of distress may be difficult to detect (Webster \& Baylis, 2000). Thus, regardless of one's feelings and emotions the fact remains that the morally distressing experience has challenged their core values and obligations. This leads to an erosion and fragmentation of one's sense of meaning and purpose in the world because sense of moral self-worth is compromised (Webster \& Baylis, 2000). For others, the experience may not be as devastating, but it does create a fissure in the self that may color future actions and decisions; for good or ill, moral residue is incorporated into their lived experience (Webster \& Baylis, 2000).

Moral distress remains a negative phenomenon that affects multiple healthcare professionals across multiple healthcare settings (Whitehead, Herbertson, Hamric, Epstein, \& Fisher, 2015). For example, a study on life-sustaining treatments found that almost half of a sample of 759 nurses and 687 physicians felt that they had acted against their conscience when providing care to terminally ill patients (Solomon et al., 1993). More recently, a study on moral distress among healthcare professionals $(n=489$ nurses; $\mathrm{n}=156$ physicians, and $\mathrm{n}=109$ other healthcare providers) in an 825-bed level one trauma center found that in ICU and non-ICU settings nurses and other direct patient care providers had significantly higher levels of moral distress than physicians and other indirect patient care providers (Whitehead et al., 2015). Direct patient care providers included nurses, physical therapists, occupational therapists, and speech therapists; and indirect patient care providers included chaplains, dieticians, pharmacists, and social 
workers (Whitehead et al., 2015). Furthermore, regardless of one's profession, healthcare providers in adult settings and healthcare providers who worked in the ICU reported higher levels of moral distress than healthcare providers in pediatric and non-ICU settings (Whitehead et al., 2015). The importance of studying moral distress relates to its pervasiveness among multiple healthcare disciplines and settings.

The ubiquity of moral distress among multiple healthcare providers reinforces the severity of the phenomenon. Critical events in healthcare call on professionals to do things that challenge their moral views, create moral distress about the action or inaction, and their inability to effect moral change for the best outcome for the patient. This is not the nurse's fault, but an indication of wrongs within the healthcare delivery system, particularly as nurses attempt to deliver safe and moral care. This deeply personal experience of moral distress is a result of healthcare professionals being prevented from doing what they perceive to be the right, moral course of action.

Morally distressing experiences and the crescendo effect impact nurses' perceptions of the quality of patient care they provide. According to Robinson et al. (2014), the Clinical Ethics Residency for Nurses (CERN) project was developed and evaluated for its effectiveness in mitigating nurses' moral distress and increasing their knowledge and self-efficacy in clinical ethics among nurses. Qualitative evidence revealed that the intervention positively impacted the attitudes, knowledge and practice of nurses $(n=67)$ who participated as evidenced by the positive program evaluations, increase in ethics knowledge, decreased moral distress, and more effective moral agency in practice (Robinson et al., 2014). Moreover, from the CERN project results revealed a positive effect on patient care as evidenced by the shared narratives provided by 
participants. For example, the participants shared narratives that demonstrated the CERN project's positive impact on their practice and patient outcomes.

Thus, as evidenced by the results of the CERN project, it may be inferred that if a clinical environment supports the exploration of ethical issues and open communication, then a positive impact is improving the quality of patient care and alleviating the moral distress nurses experience (Robinson et al., 2014). However, if moral distress is left unaddressed, then the crescendo effect may develop. This study demonstrated that the crescendo effect would in fact develop, as evidenced specifically in two surgical ICUs (Surgical Intensive Care Units or SICUs), if morally distressing events compound into unresolved moral residue and subsequently a crescendo effect develops. It is unknown whether frequency or intensity of moral distress plays a greater role in transforming moral distress into moral residue over time. In addition, it is unclear how timing, acknowledgement of distress, and/or coping methods influences development of moral distress and moral residue. In this dissertation study, these perceptions and experiences of moral distress, moral residue, and coping with stress employed by SICU nurses, along with their perceptions of the quality of their patient care were explored.

Understanding how diverse experiences manifested outwardly and inwardly may assist in identifying and potentially alleviating those experiences and the underpinning moral issue(s). Identifying the impetus for the moral distress experience retained the focus on not only helping nurse's identify and alleviate personal experiences of moral distress, but also focus on mitigating moral issues that occur in patient care to advocate for the patient's quality of care. 
The nurse participants' responses to moral distress was important in this study because whether it was an outward or inward response, the occurrence of moral distress was worth investigating in order to determine the severity of the nurse participants' moral residue. Moral distress may affect nurses differently, and may feel that their compromised values and integrity inculcate a sense of culpability to their nursing practice. Whether or not they show their experience outwardly or, feel negative emotions inwardly, the moral issue still challenges their moral integrity, particularly in a way that impacts how they care for their patients. Underpinning moral distress potentially impacts a patient's quality of care. Therefore, the development of moral residue is a stimulus for nurses to know with greater clarity what they will tolerate or cooperate with in future instances where moral issues threaten patient safety and quality of life (Webster \& Baylis, 2000). According to Webster and Baylis (2000), the change is negative when the person adapts to moral challenges by constantly shifting their values whereupon their values become so adaptable that it is nearly impossible for the person to articulate what they sincerely believe. This capitulation ultimately leads to desensitization to wrongdoing (Webster \& Baylis, 2000), which in the SICU healthcare context-where margin for error is minimal—represents a diversion from patient safety and quality patient care.

Different coping mechanisms influenced nurses' perceptions of the intensity of morally distressing events. Therefore, this study is unique because, SICU nurses were asked about their coping, moral distress, moral residue, and their perceptions and experiences of quality patient care were not examined until this study. Exploring various coping strategies assisted in illuminating how moral distress and moral residue was perceived and experienced by the nurse in the SICU. Understanding coping mechanisms 
by exploring underlying moral issues may also help researchers develop interventions aimed at improving nurses' coping abilities and mitigating the occurrence of moral distress and moral residue in the practice environment. Understanding perceptions of quality patient care may also help researchers target ways to identify and link moral issue(s) with strategies both to decrease moral issues and to increase nurses' abilities to cope and manage their morally distressing experiences in a way that does not rob patients of their quality of care in a complex ICU environment.

Prolonged exposure to moral distress resulting in moral residue may lead to an alteration in the way an individual nurse approaches ethical patient care situations. Feelings of powerlessness stem from complicity in perceived immoralities (i.e., aggressive care at the end-of-life would be an immorality if it is prolonging the patient's life, against the patient's values and wishes, and causes pain) and an inability to effect change (i.e., the nurse is unable to convince the family and/or healthcare team to stop aggressive care in futile situations). The nurse is morally distressed because they perceive the delivery of poor quality of care from their moral intuitions and moral evaluations (Dudzinski, 2016; Webster \& Baylis, 2000). Thus, nurses become complicit in these initial experiences, which may include working quickly, being lazy, or even cowardly, or simply the perception that they are involved in some type of ethical error in patient care (Dudzinski, 2016).

This perception is important to recognize because the nurse's personal and professional moral integrity along with their core obligations and values are challenged. Poor quality of care becomes the focus because the primary moral goal is not to mitigate the nurse's experience of moral distress where they feel challenged ethically, but to 
identify and address the moral issue(s) that cause that perception and experience (Dudzinski, 2016). Moral distress is caused by a perceived immorality because the nurse is unable to change the course of action. Regardless of how negative and emotional the experience is outwardly and/or inwardly for the nurse, the fact remains that a moral issue prompted the nurse's response. Thus, it is essential to address the moral distress in nurses to prevent or mitigate future occurrences.

As these instances occur over time without acknowledgment or reconciliation by the individual nurse or unit/team, the potential impact on quality of patient care and potential for error must be examined. Moral residue as a result of cause of error can result from the incremental loss of commitment to previously held values for reasons of selfinterest, including self-protection or self-promotion (Dudzinski, 2016). Theoretically, the experience of moral distress leads to perceived poor quality of care when: (a) nurses' initial moral distress and subsequent powerlessness to take the moral course of action results in an awareness of complicity in providing poor quality of care; and (b) during the crescendo effect, when the nurse provides poor quality of care due to withdrawal and burnout from the ethical dimensions of practice. Thus, this study explored how the crescendo effect was, in fact, dependent on the nurse's exhausted moral integrity and sustained powerlessness with respect to moral distress compounded by stains of moral residue.

According to the crescendo effect, it is theorized that one can only take so much distress before it begins to impact their work/life balance and pile-up and result in some tipping point over the edge of manageability. This study explored how this pile-up impacts SICU nurses' coping and also perceived quality of patient care. Similarly, the 
pile-up of stressful events influenced coping ability. SICU nurses' perceptions of their quality of patient care was influenced by their firsthand morally distressing experiences and subsequently becoming detached and withdrawn in their practice over time.

In summary, the experience of moral residue can be the result of error or the cause of error. Moral distress and moral residue are intertwined as concepts and require further investigation to illuminate their close interrelationship. These interrelationships eventually lead to the crescendo effect, which may result in conscientious objection, distancing one's self from a particular patient or caring task, burnout, and withdrawal from the nursing profession (Epstein \& Hamric, 2009).

It is unclear if the progression of moral distress to moral residue leading to the crescendo effect is a linear process. However, the timing of how these concepts unfold is not supported by current evidence. To the best of this researcher's knowledge, data on the perceptions and experiences of specifically adult SICU nurses as it applies to moral distress, moral residue, the crescendo effect, stress and coping, and perceptions of quality patient care are largely left unaddressed in the scientific literature.

Therefore, the purpose of this descriptive qualitative study was to explore nurses' perceptions and experiences of moral distress and moral residue in the adult SICU environment in order to determine root causes of moral distress; perceptions and experiences associated with moral distress, moral residue, stress and coping, and perceived quality of patient care. By exploring these concepts among SICU nurses while identifying the moral issues that lead to moral distress a better understanding of the phenomenon was achieved, leading to insights essential to the development of future interventions to address moral distress and moral residue and improve patient care. 
Unique to this study is the exploration of nurses' coping abilities in the midst of moral distress and moral residue. Interestingly, Henrich et al. (2017) found from 10 focus groups with 56 participants (nurses and healthcare professionals) in 13 ICUs in the Vancouver area of British Columbia, Canada, that coping with emotions through venting, debriefing, and discussing the morally distressing situation with compassionate colleagues were successful methods to manage the negative emotional responses to moral distress. Furthermore, Henrich et al. (2017) revealed that nurses and other healthcare providers frequently maintained that it was best to address the cause of the morally distressing critical event. This is critical information that this study considered because identifying the original moral issue that created moral distress will help healthcare professionals mitigate the occurrence of moral wrongs in the clinical environment. Identifying nurses' coping abilities with moral distress is also an important concern for researchers. According to Bruce, Miller, and Zimmerman (2015), maladaptive coping with moral distress included pas-de-deux, fighting, and withdrawing, while constructive coping included methods such as venting, mentoring networks, and building team cohesion. The specifics of Bruce et al.'s (2015) study has implications for this study and will be discussed later in greater detail; however, it is important to note that coping is a part of moral distress research, because nurses may go through a series of approaches to decrease moral distress before becoming withdrawn from the ethical dimensions of their practice. Moreover, Lievrouw et al. (2016) studied coping with moral distress in the oncology setting and found that four dominant ways of coping emerged: thoroughness, autonomy, compromise, and intuition. It was also revealed that physicians and nurses had a tendency to internalize or externalize moral distress and also a 
propensity to focus on rational or experiential elements (Lievrouw et al., 2016). It is important to understand the symptoms of moral distress because it provides insight into how damaging the phenomenon is to nurses who experience its negative effects.

We may gain an understanding of the psychological harm of moral distress and methods of coping, but central to mitigating the occurrence moral distress in the healthcare system is to acknowledge that moral issues occurring within the system stem from root causes. The concept is an alarm because clinicians are not to blame, but rather the environment in which they are providing care creates moral distress, as well as clinical situations, units/teams, and systems/organizations.

A combined individual, team/unit, organization/system approach is warranted in order to combat the systemic nature of this problem in healthcare (Hamric, 2014). For example, if the nurse attempts to address their moral distress but is met with barriers from unit/teams, and/or organizations, then moral residue will build and the vicious cycle of inaction and immorality will continue. However, achieving an understanding of individual-levels of moral distress and moral residue and how these unresolved feelings stemming from violations to core values and duties impacts nurses' perceptions and experiences, may subsequently provide insight into tailoring interventions to help change the culture of healthcare by directly targeting moral issues.

\section{Theoretical Framework}

Epstein and Hurst (2017) aptly describe moral distress as a system problem that nurses experience and should alarm the broader healthcare team in order to rectify moral wrongs. Blaming nurses for their experience of moral distress and moral residue negates their professional standards of practice, values, and personal moral integrity. Moral 
distress remains a systems-level problem. Ignoring nurses' experiences of moral distress and moral residue, or blaming them for being overly sensitive to the critical events of practice is unacceptable. Instead, addressing the moral distress problem by exploring nurses' perceptions and experiences and acknowledging the root causes of moral distress and unresolved moral distress (moral residue) will uncover the systemic nature of this negatively pervasive healthcare problem. Only by addressing underlying moral issues will any meaningful dialogue be achieved, moving the state of the science forward by intervening on behalf of moral issues that occur in practice and impact quality patient care.

How SICU nurses cope with their frustration and powerlessness when morally distressed and manage their professional and personal obligations was of interest in seeking to: (a) advocate first and foremost for the patient's best interests and quality of care; (b) advocate for nurses' well-being through an ethical and healthy work culture; (c) maintain dialogue about ethical dimensions of practice that consider nurses, other healthcare professionals, families, and patients in reciprocal interrelationships of caring and understanding in order to avoid discounting diverse perspectives; (d) retain nurses that feel valued and respected; and (e) provide quality patient care.

A combination of two theories informed and supplemented this body of research. Lazarus and Folkman's (1984) Transactional Model of Stress and Coping combined with Epstein and Hamric's (2009) Crescendo Effect informed this study, because moral distress is a negative response that manifests as powerlessness, anger and frustration (Hamric et al., 2012) and depends on temporal, contextual, and situational specific factors in the ethical dimensions of practice (Epstein \& Hamric, 2009; Hamric, 2014) that are 
potentially influenced by how nurses respond in the caring relationships between patients, families, and other healthcare professionals. The theoretical framework for moral distress and moral residue was represented by the crescendo effect and conceptually underpinned this study (see Figure 1 in Appendix A). Furthermore, stress and coping theory (Lazarus \& Folkman, 1984) also informed this framework in order to investigate how nurses cope with varying levels of stress incurred by participating in perceived immoral actions at the bedside through emotion- and problem-focused coping. The theoretical model of the experience of moral distress and moral residue provided a sensitizing framework for this study.

\section{Crescendo Effect}

Moral distress is a grave and systemic problem in healthcare. Moral distress is defined as the inability to do the perceived right course of action in a clinical situation due to a myriad of internal and external constraints that prevent action in accordance with one's professional standards of practice and personal moral integrity. These constraints may manifest as power differentials, medical futility, inadequate resources, and lack of staff, which challenge providers in their ability to provide care that is in the best interests of the patient's quality care. Moral distress is so morally distressing because bedside level provider's experiences are not being acknowledged and addressed through institutional policies and procedures, which is particularly salient among critical care nurses given the work environment they endure (Whithead et al., 2015). According to Hamric and Blackhall (2007) $45 \%$ of 90 ICU nurses said they left or considered leaving a position due to moral distress. This provides an indicator of the moral issues occurring in the healthcare system, because nurses are leaving the high stress environment of the ICU due 
to the moral distress and moral residue that it creates. Moral distress and moral residue are a problem for those that experience it as well as the actual sources of that distress, because the distress indicates broader moral issues situated within the healthcare system that require remedy. Importantly, the crescendo effect theoretical model is about the interrelationship between moral distress and moral residue over time. This study explored the perceptions and experiences of SICU nurses in order to assess the applicability of the crescendo effect to firsthand accounts of moral distress, moral residue, coping, and quality of patient care.

The manifestations and meaning of moral distress and moral residue may change in different settings and time periods. The crescendo effect (see Figure 1 in Appendix A) is a model that hypothesizes that moral distress builds over time and eventually becomes unmanageable, thus moral residue is dependent upon repeated experiences of moral distress (Epstein \& Hamric, 2009). The first crescendo is moral distress and as a morally distressing event concludes the nurse may note some relief and decrease in moral distress. Root causes continue to occur and moral residue develops, which eventually crescendos because distress and unresolved moral issues have piled up in the individual to the point that reconciliation is not feasible. Moral distress and moral residue are assumed to be context-and situation-specific, and influenced by time (Epstein \& Hamric, 2009). The gaps in moral distress knowledge are exemplified in the following questions posed by Epstein and Hamric (2009): How many years or exposures does it take to build a professionally noticeable moral residue crescendo? Do some types of critical events raise moral distress more than others? Is the crescendo pattern necessarily linear?” (p. 10). This framework provides another way to guide the study the timing of moral distress in 
individual nurses. This researcher explored adult SICU nurses' years of experience in order to understand how length of time in the SICU may contribute to moral residue. In addition, clinical situations that lead to moral distress in adult SICU nurses were investigated.

Perhaps it is still unclear if the crescendo pattern is linear or not, meaning that it may be the result of one critical event or an accumulation of multiple unresolved clinical events that manifest as powerlessness and frustration. Thus, adult SICU nurses in this study were asked about past and present practice in order to explore the manifestation of moral distress and moral residue. By studying the individual nurse in this way and cataloguing how, what, and when moral distress occurs one may readily identify root causes of moral distress (i.e., moral issues that burden the conscience of nurses) more effectively. These root causes may be acknowledged at an individual-level, highlighting how it is insidiously allowed to continue at the unit- and systems-level, because of lack of acknowledgement.

Nurses' voices ought to be listened to, because they spend the most time with patients at the end-of-life than any other healthcare professional. If they feel so negatively about a course of treatment and moral distress is documented so widely in research, particularly in the critical care adult environment (Whitehead et al., 2015), then this impact on the patients' quality of care ought to be a primary concern. Moral distress will never be completely eradicated (Hamric, 2014) due to technological advancements that prolong life and make quality of care determinations difficult. As technology continues to advance, our ethics must keep up with attempting to address the gray areas of healthcare practice through a constant exploration of the morality of individual actions and 
consequences. However, if the environment in which moral distress occurs is changed and structures that perpetuate moral distress are challenged, then the charge of identifying the immorality of patient care may be front and center at the delivery of care as it ought to be when considering the ethical dimensions of practice aimed at providing for ailing, vulnerable patients in need of quality care.

This study is different from current research in several ways. Bruce et al. (2015) used qualitative case studies to interview ancillary staff (i.e., four social workers, two chaplains, and a case manager) and clinicians (i.e., 16 nurses, three surgeons, and three intensivists) from a medical and surgical adult ICU in a 900-bed academic hospital. The questions probed sources of moral distress; how it impacted participants and their patient care; helpful or unhelpful interventions used to ease moral distress; relationships involved; and whether anything could have been done differently. Three types of dissonance led to moral distress: (a) a disagreement between a healthcare professional and a patient or decision maker; (b) discordance between patients/family members and other family members; and (c) disagreement among healthcare professionals regarding a patient's care. This latter source of intrateam disagreements was cited most frequently and was created through initiation or maintenance of non-beneficial life-sustaining treatments and a lack of full disclosure about treatments resulting in inadequate informed consent.

According to Bruce et al. (2015), participant characteristics were biased due to gender, because the ICUs were primarily populated by male physicians and female nurses. A limitation of their research is the lack of male nurses. Specifically, the data from this study suggests emotional nurses and a potential limitation is the revelation that 
physicians and nurses differ in how they perceive and experience immoralities. However, according to Bruce et al. (2015), attribution of their findings is most likely a result of unit culture and differences in professional backgrounds, rather than gender differences.

This study explored the moral issues that cause moral distress in adult SICU nurses, because it may be theorized that if SICU nurses had more maladaptive coping than medical ICU nurses in Bruce's et al. (2015) study then it is worth exploring why this is so. Specifically, since Bruce et al.'s (2015) research demonstrated that SICU nurses employed fewer constructive coping methods, this unit-specific environment is of interest. The SICU is of interest, because it is unclear if the culture is causing the lack of constructive coping, the background of the nurses, it may be the nature and type of patients they treat, or some other unknown reason yet to be discovered. This uncertainty in and of itself demonstrated a need for this study, since its sole focus is on adult SICU nurses and how they cope with moral distress and moral residue. Also, a critique of Bruce et al. (2015) is that they did not explore moral residue and how it factors into the experience of moral distress over time. This study filled this gap by individually interviewing SICU nurses working in two different SICUs in one trauma facility. According to Bruce et al. (2015), how healthcare professionals managed their moral distress was grouped into two categories: maladaptive and constructive behaviors. Specifically, maladaptive coping mechanisms included pas-de-deux, fighting, and desensitization. For example, pas-de-deux is a way of reconciling multiple forms and sources of prognostic information and plans of care (Bruce et al., 2015). Specifically, two approaches to reconcile communication with disparate clinical perspectives included (a) maintaining superficial conversations with families so that no one could object to the 
content; or (b) having substantive goals-of-care conversations with patients or families, but accounting for divergent views by using phrases like, but others may disagree, we are still not sure, and let us wait and see.

Interviewees reported fighting or confronting the healthcare professional who was in discordance with other healthcare team members (Bruce et al., 2015). In particular, words used by an intensivists and three nurses included "posttraumatic stress injury," "death row," "combat," "battle," and "war" (Bruce et al., 2015). Desensitization was found to persist when fighting failed, because 18 interviewees expressed feeling “ineffectual” (Bruce et al., 2015). Interestingly, four of the interviewees who described feeling ineffectual were men and they had a range of experience from 2 to 26 years (Bruce et al., 2015). Thus, the study findings support the notion that healthcare professionals may withdraw or detach after repeated experiences of feeling ineffectual to protect their professional integrity (Bruce et al., 2015).

Constructive behaviors were also noted in the management of moral distress and included venting, mentoring, and building team cohesion. Interestingly, no one from the SICU in the Bruce et al. (2015) study mentioned team cohesion or how it could be used to counterbalance moral distress. Thus, the importance of this current study cannot be understated because it explores the stress and coping mechanisms of SICU nurses in managing and addressing morally distressing experiences.

This current study sought to include male nurses and will also only be investigating adult SICU nurses - no other professions will be included. Thus, this study strived not to skew data in terms of gender. Furthermore, perhaps constructive coping mechanisms were not found in the SICU because Bruce et al. (2015) only interviewed 15 
SICU nurses in one SICU. However, this study filled this gap by interviewing adult SICU nurses across two SICUs.

Stress and coping is a part of this study's theoretical framework, thus Bruce et al.'s (2015) study informed this study's theoretical framework. For example, the coping in Bruce et al.'s (2015) study was dichotomized into constructive and maladaptive coping mechanisms. This information has applicability to this study, specifically related to the types of coping that are considered beneficial and harmful. This earlier work informed the current theoretical framework for this study by considering that all coping is good, because it is an attempt to deal with moral distress; however, the outcome for the patient and family may not always be good.

A larger sample of heterogeneous nurses recruited from multiple SICUs is necessary to accurately document the occurrence of moral distress and moral residue and the various forms of coping used. However, it is also worth noting that if a nurse does not feel that they experience moral distress then it needs to be investigated in order to acknowledge and understand that perception and experience. The benefit of qualitative descriptive research is to explore and understand the subjective perceptions and experiences of participants. It is necessary to better understand whether those perceptions are influenced by singular experiences, or as detached, multiple experiences, which are then left unresolved and contribute to a crescendo effect. In addition, disparate perspectives in the literature exist regarding whether the frequency or intensity of morally distressing experiences are more damaging. This study explored this nuance of moral distress and moral residue. Also, the perception of reduced quality of care among nurses was present and also led to care that was perceived to be poor. Thus, unpacking these 
perceptions and experiences support a more informed model of the crescendo effect along with the influence of stress and coping, which this study accomplished. Importantly, Bruce et al. (2015) noted that SICU nurses experienced the most unrelieved moral distress. This could be from a multitude of reasons, not the least of which is limited to: the age and diagnosis of the patient, the patient and/or family's length of stay, and the disposition of the nurse and other members of the healthcare team. Therefore, exploring this niche population of nurses is crucial in understanding the implications of moral distress and moral residue for nursing research, practice, and policy.

Robust emotional reactions from morally distressed nurses support the crescendo effect. According to Epstein's (2007) hermeneutic phenomenology dissertation in which healthcare professionals were interviewed six weeks after the death of infants in a newborn ICU, moral distress among physicians was less emotional than among nurses, but it was a problem because physicians still verbalized powerlessness. Thus, just because a healthcare professional occupies a particular role in the clinical setting does not mean that they will fit a mold for a particular response to moral issues. Similarly, this study's investigation of nurses' perceptions and experiences was vital to the exploration of a complex phenomenon like moral distress that is diverse in its manifestation.

Determining a pattern or lack thereof to moral distress and moral residue, and its eventual manifestation physically and emotionally in an individual is something this study uncovered. Thus, it was worth exploring whether or not maladaptive responses to moral distress and moral residue evolve and coalesce into burnout or simply a jaded sense of self that leads to poor quality of care. Bruce et al. (2015) noted that future research might allow interventions to target nurses based on a spectrum of moral distress: initial 
onset to chronic. This supports Epstein and Hamric's (2009) crescendo effect and the important need for this study. According to Wilson, Goettemoeller, Bevan, and McCord (2013), their exploratory study surveyed 50 nurses from a medical-surgical ICU using the MDS, and found low levels of moral distress. However, nurses' open-ended comments revealed that moral distress impacted their coping strategies, and their personal and professional lives. This demonstrates an important nuance regarding moral distress: nurses may report a lack of moral distress, but act differently when they go home, ruminate on the experiences from work, and are not under the influence of the clinical environment.

This study was limited in studying the interrelationship of moral distress and moral residue over time because qualitative interviews will occur at one time and place. Therefore, it is not possible to assess and evaluate individual experiences over a continuum of time. However, questions asked by the interviewer at the time and place may retrospectively explore how the nurse's relationship with moral distress and moral residue has or has not changed, evolved, or developed into their current perceptions and experiences that underpin how they cope and perceive the quality of their patient care.

Nurses and nurse practitioners with two to five years of experience had greater moral distress than those with less than two years of experience (Johnson-Coyle et al., 2016). Perceived moral distress was the result of intensity, rather than frequency. Previously, Epstein and Hamric (2009) stated that it is unclear how time and intensity of moral distress contribute to a crescendo effect. Johnson-Coyle et al.'s (2016) findings demonstrated that intensity of moral distress plays a bigger role. Discrepancies regarding the impact moral distress has on nurses necessitates the clarity this study provides. For 
example, frequent and disturbing clinical situations that created moral distress occurred in the SICU, contributed to the crescendo effect and also impacted perceptions of quality patient care. Hamric and Blackhall (2007) found that total moral distress scores were driven primarily by RNs' perceptions of the frequency of morally distressing situations. For example, even though RNs and medical doctors were both distressed by the provision of futile treatment, RNs perceived that it happened more often than doctors.

Moreover, DeKeyser and Berkovitz (2012) found from their study of 119 surgical nurses that lower levels of general quality of nursing care, nursing skill, and being able to meet the patient's needs were associated with higher frequencies of ethical dilemmas/moral distress. However, the intensity of these feelings was not found to be related to any aspect of quality of care. As demonstrated by Hiler (2018), it seems that the intensity of moral distress does not relate to perceptions of quality of care, only frequency of those experiences. Thus, current research indicates dissonance regarding what experience of moral distress, the frequency or level of disturbance/intensity, tends to drive negative experiences as moral issues occur in practice. This underpins the importance of this study in identifying the interrelationship between moral distress and moral residue and how a combination of the frequency and intensity of those experiences drives coping mechanisms and perceptions of quality patient care.

An exploratory, descriptive study was conducted to conceptually clarify and contextually understand moral distress in the care of patients living with dementia in residential care (Spenceley, Witcher, Hagen, Hall, \& Kardolus-Wilson, 2017). Results indicated six themes related to moral distress: conflicting expectations around care, remaining silent, failure to be accountable for care, failures of leadership, the wrong 
model of care, and a culture of work that privileges tasks over touch (Spenceley et al., 2017). Some co-workers were noted by nurses to rush their care, which also resulted in errors. Spenceley et al. (2017) demonstrated that quality patient care is impacted by the experience of moral distress. Specifically, their study revealed that the experience of moral distress was perpetuated by more than just one's experience, but also from the structure of the unit and organization, which supported the systemic nature of moral distress according to Hamric (2014) because it occurs at all levels: the individual/patient, unit/team, and organization/system.

\section{Stress and Coping}

This study will also use Lazarus and Folkman's (1984) Transactional Model of Stress and Coping to inform how nurses use emotion- and/or problem-focused coping to mitigate moral distress and moral residue. Emotion-focused coping is defined as a way of appraising and coping with a situation through emotional regulation (Lazarus \& Folkman, 1984). Thus, the particular situation is judged as requiring acceptance (Lazarus \& Folkman, 1984). Emotion-focused factors include seeking emotional social support, distancing, avoiding, emphasizing the positive features of a situation, and self-blame (Lazarus \& Folkman, 1984). For example, denial is a form of emotion-focused coping, but it may allow for problems to be solved (Lazarus \& Folkman, 1984). Specifically, denial in the case of staffing shortages in the SICU may be a primitive defense to mitigate dwelling on the fact that there are unlimited needs and limited resources to deal with them; however, the nurse is able to use denial to preserve her emotional balance required to engage in the problem solving activities of patient care. Alternatively, problemfocused coping is defined as a way of appraising and coping with a situation by 
attempting to relieve the problem by doing something (Lazarus \& Folkman, 1984). Thus, the situation is judged as something that can be acted on and solved (Lazarus \& Folkman, 1984). Problem-focused factors include cognitive and behavioral problem-solving strategies, such as attempting to come up with multiple solutions to a problem, collecting information, and developing an actionable plan to follow (Lazarus \& Folkman, 1984). Interestingly, it is difficult to conclude whether a particular strategy is emotion- or problem-focused, because any action or thought can have multiple coping functions (Lazarus \& Folkman, 1984). Thus, an attempt to determine whether emotions are being regulated or problems solved becomes impossible (Lazarus \& Folkman, 1984). For example, consider a nurse that drinks alcohol to cope with the moral distress at work. If the nurse takes alcohol to control her moral distress, then it will interfere with her ability to care for patients. However, she is problem solving by trying to manage her feeling of distress. Alternatively, a nurse may be extremely meticulous and study to gain critical care certification so that she gains control of feelings of inadequacy in order to master her profession so that she is safe and competent in the delivery of her care. In this latter case, the fear of hurting a patient or performing badly is managed by problem-focused coping. In addition, a high level of threat does not mean that either or both forms of coping will diminish in quality (Lazarus \& Folkman, 1984). For example, individuals coping with stress related to physical illness, disability, and warfare or plane crashes demonstrate high-level emotional and cognitive functioning, which are potentially the most difficult of circumstances (Lazarus \& Folkman, 1984). Thus, coping behavior is multi-determined and the level of the threat is only one factor (Lazarus \& Folkman, 1984). 
It is important to use this framework to tease out that moral distress is not the same as psychological distress because it is absent a moral component, but it is also necessary to delineate what stresses providers out about the moral issues occurring in their practice. Emotion- and problem-focused coping will inform this study, because it is unclear what methods or combination of methods critical care nurses routinely use to manage their moral distress experienced in the ICU environment and whether it contributes to moral residue, burnout, and/or improving or hampering the quality of patient care they provide. Furthermore, it is worth exploring nurses' coping with moral distress in order to determine if they actually address it at the unit/team and organizational level by contacting management and individuals in positions of authority. If they do address moral distress, it would be interesting to note through what method and if not: why did they choose not to address it. Also, if not, then it would be worthwhile to explore what would be a good way for them to start, provided they have the encouragement and opportunities at their disposal.

Epstein and Hamric (2009) described three coping strategies seen in the literature as withdrawal from the ethical dimensions of practice, conscientious objection, and burnout. While an objective empirical link is missing regarding these consequences, their pervasiveness in the moral distress literature is undeniable and warrants further qualitative exploration. Furthermore, research on the following concepts' interactions with moral distress is ambiguous: moral resilience, ideology, spirituality, compassion fatigue, and knowledge of ethics. Additionally, Wilson et al. (2013) cited ethics committees and debriefings as resources used to cope with moral distress. Since individual nurses' perceptions and experiences will guide the data obtained in this 
qualitative study, it is unknown whether all of these concepts and potential avenues of coping will be addressed in detail. Instead, the participant's firsthand accounts will guide data development in an attempt to obtain a subjective account of one's individual perceptions. Specifically, this study focuses on an exploration of perceptions and experiences of moral distress, moral residue, coping, and quality patient care.

The Transactional Model of Stress and Coping, "views the person and the environment in a dynamic, mutually reciprocal, bidirectional relationship" (Lazarus \& Folkman, 1984, p. 293). Moral distress is a process that unfolds in an ever-changing environment where the nurse's response is also constantly changing as well as their relationship to the environment (Lazarus \& Folkman, 1984). In this process, coping may or may not occur. Also, where the coping takes place is also important, because if it occurs in the clinical setting, then determining if the moral issue is addressed is of interest. If coping takes place at home, then it would be worth exploring if it is constructive or maladaptive. According to Lazarus and Folkman (1984), emotion-focused coping is likely when a situation's challenges are appraised to be non-modifiable, so strategies include minimization, distancing, and positive comparisons. For example, a nurse may say things could be worse or imagine herself in another situation to ignore the moral issues in her practice. An example in the moral distress context includes a clinical situation where non-beneficial, aggressive treatment at the end-of-life is occurring. Moral distress creates a feeling of powerlessness to act and do the right thing. Thus, emotionfocused coping in a moral distress context of non-beneficial, aggressive treatment includes ignoring the issue and continuing to provide treatment without questioning the healthcare team, despite the perceived wrong by the nurse and powerlessness, too. 
Problem-focused coping occurs when a situation is appraised to be modifiable, and this analytic process may be directed outwardly and inwardly (Lazarus \& Folkman, 1984). Externally directed problem-focused coping strategies include altering pressures and barriers to change, while internal efforts include motivational changes and developing new behaviors, or learning new methods to handle barriers (Lazarus \& Folkman, 1984). In a moral distress context, an example of an externally directed problem-focused coping strategy was mentioned in the Bruce et al. (2015): venting, mentoring, and building team cohesion. Thus, the nurse may alter pressures and barriers to change by engaging in teamwork in order to minimize the impact and duration of moral distress. Also, in a moral distress context, an example of an internally directed problem-focused strategy includes motivational changes and developing new behaviors, such as exercising, journaling, meditation, and the use of drugs and/or alcohol (this is not a positive coping mechanisms, but it demonstrates an example of internally directed problem-focused coping).

There is a limited amount of nursing research addressing the coping that nurses use in response to moral distress. Henrich et al. (2017) found from focus groups with 56 participants in 13 ICUs, that coping through venting, debriefing, and discussing moral distress with colleagues were methods to manage the negativity. Specifically, across all provider types (i.e., staff nurses, clinical nurse leaders, other healthcare professionals, and physicians) in this study, frustration was the most frequently described emotion associated with morally distressing experiences (Henrich et al., 2017). Coping strategies included, "requesting to change their patient assignment, taking a break from the ICU and working in a general care area, and drinking alcohol after work" (Henrich et al., 2017, p. 
e51). Coping with emotions from moral distress were achieved through venting, discussing the situation with compassionate colleagues, or debriefing with their healthcare team (Henrich et al., 2017). Healthcare professionals noted that they had learned to cope with moral distress over time, and this experience allowed them to compartmentalize their emotions, while other became less invested in their work so they could distance themselves from the morally distressing experiences (Henrich et al., 2017). In addition, some nurses and other healthcare professions noted that the ICU culture prohibited sharing emotions, thus staff repressed and hid their experiences with moral distress to not appear weak (Henrich et al., 2017). Some strategies to cope with the roots of this distress included requesting a change of patient assignments, taking a break from the ICU and working in another care area, and drinking alcohol after work (Henrich et al., 2017).

Interestingly, moral distress was noted to have a negative impact on care (Henrich et al., 2017). For example, all provider groups stated that dealing with morally distressing experiences demanded their time and attention (Henrich et al., 2017). Nurses reported that basic care needs were in jeopardy, because they had to spend time advocating for their patients (Henrich et al., 2017). Also, other healthcare professionals, stated that their other patients get neglected when they are dealing with a patient in a morally distressing situation (Henrich et al., 2017). Thus, these healthcare professionals avoid situations to save time, but this avoidance may result in a lack of support for families and patients, or poor quality of care (Henrich et al., 2017). Participants revealed that feeling morally distressed caused them to be distracted or unfocused (Henrich et al., 2017). Also, other healthcare professionals stated that having their opinions and input ignored in the past 
may decrease their willingness to provide their opinion in the future, which could also negatively affect patient care (Henrich et al., 2017). Thus, understanding the symptoms and responses to moral distress provides insight into its damaging effects, because the causes are moral issues that may underpin broader wrongs occurring in the healthcare system.

Importantly, Henrich et al.'s (2017) study has important implications for this study. A weakness and limitation of Henrich et al.'s (2017) study was the use of focus groups and also phone interviews to gather data when staff were unable to attend focus group meetings. Henrich et al. (2017) noted a limitation of their focus groups is a lack of generalizability. However, the purpose of qualitative research is to highlight the perceptions and experiences of participants, whether they be unique or similar. The idea is not to seek generalizability, which makes one question the methodological rigor of their study. This study addressed this weakness by conducting individual, one-on-one interviews with SICU nurses in an effort to prevent participants from just providing socially desirable responses, which may be a consequence of focus groups because of the public forum in which it takes place. Also, the number of staff nurses and clinical nurse leaders were outweighed by the participation of other healthcare professionals and physicians. Thus, this study only focused on nurses.

Stress and coping was a part of this study's theoretical framework, thus Henrich et al.'s (2017) study supported this study's theoretical framework. For example, some of the coping mentioned by participants in Henrich's et al.'s (2017) study may have applicability to this study, specifically related to types of coping, such as coping with emotions from moral distress through venting, discussing the situation with 
compassionate colleagues, or debriefing with their healthcare team. The aforementioned methods of coping mechanisms were similarly associated with morally distressing experiences in this study, but it remained important to be theoretically sensitive and not lead participants to answers, but rather let the data speak for itself and convey meaning through the participants' perceptions and experiences.

Thematic analysis focusing on critical incidents among 17 physicians and 18 nurses working in three different oncology hospitals was used to explore ways of managing moral distress, how responses evolved throughout careers, and how training and team culture impacted those experiences (Lievrouw et al., 2016). Relationships were graphically displayed between different themes, leading to a model that depicted four dominant ways of coping with moral distress: thoroughness, autonomy, compromise, and intuition, which were either internal or external in their manifestation on the x-axis and included a tendency to focus on rational elements or experiential elements on the y-axis. In particular, the four dominant ways of coping with moral distress comprised each quadrant in the two-dimensional model. Thus, experiences of moral distress were noted to have four different ways of coping, but existed by either internalizing or externalizing the experience. Internalizing moral distress was the feeling of being personally involved in a moral conflict (Lievrouw et al., 2016). Externalizing one's coping with moral distress is characterized by dis-ownership, turning away from the question of one's own values and norms, and focus on causes beyond one's control (Lievrouw et al., 2016). Thus, these types of healthcare professionals prefer quick solutions (Lievrouw et al., 2016).

On the y-axis of the model, a tendency to focus on rational elements exists at one end of the spectrum and the other end is denoted as a tendency to focus on experiential 
elements. Interviewees that showed a predisposition to focus on rational elements when dealing with a morally distressing experience primarily paid attention to theoretical and academic rationales, processes, and structures (Lievrouw et al., 2016). For example, they would rather have well-structured formal deliberation to smooth out a morally distressing experience (Lievrouw et al., 2016). However, focusing on experiential elements meant there was a strong concerns about the experiences and feelings of those involved (Lievrouw et al., 2016). Thus, a preference for experience-based learning and team discussions were preferred along with informal ways of handling moral distress (Lievrouw et al., 2016). When the axes of rationality, experience, internalization, externalization merge into a single model they exemplify not one preferred method of coping over another, but rather a way to characterize dominant ways of coping, with each possessing its own degree of strengths and weaknesses, and implied preferred solutions and learning styles (Lievrouw et al., 2016).

Lievrouw et al.'s (2016) study has important implications for this study. A weakness and limitation of Lievrouw et al.'s (2016) study is the point they made that the findings cannot be generalized to other settings or caregivers. However, this is a known consequence of qualitative research and is a moot point. Furthermore, the data reflected in this small number of nurses and physicians took place in the oncology setting. However, this study only addressed SICU nurses, particularly since this population demonstrated in Bruce et al. (2015) that they did not have any constructive coping mechanisms.

Stress and coping is a part of this study's theoretical framework, thus Lievrouw et al.'s (2016) study supported this study's theoretical framework. For example, participants 
in Lievrouw et al.'s (2016) study had a tendency to either internalize or externalize their experiences of moral distress and also focus on rational or experiential moral distress elements. Thus, this prior theoretical work informed this study because some of these coping characteristics were described by adult SICU nurses. However, it remained important to be theoretically sensitive and not lead participants to answers, but rather let the data speak for itself and convey meaning through the participants' perceptions and experiences. Also, Lievrouw et al. (2016) created a theoretical model of moral distress coping strategies from their results. The goal of this study was to explore the perceptions and experiences of adult SICU nurses, not create a new theoretical framework. Instead, prior research was used to inform a process of moral distress, moral residue, and the crescendo effect - including stress and coping, and perceptions of quality patient carethat this study explored. This process model was born out of the results of this study, and is also a way of describing the phenomenon up to this point. It is important to note that this study did not attempt to fit the current theoretical model to the interview process, but rather let the participants and their experiences and perceptions drive the data.

Each nurse reacts differently, thus exploring the phenomenon across multiple ICUs is warranted. Physicians preferred a rational coping style while nurses focused on their feelings (Lievrouw et al., 2016). This is consistent with Bruce et al. (2015) identifying that physicians focused on science while nurses on humanistic elements. It is difficult to say whether or not these methods of coping are problem- or emotion-focused coping. For example, if nurses become emotionally invested then it may be stated that this is emotion-focused coping. However, if this type of coping is done in order to maintain a human connection to the patient and thus provide care that is moral, then in 
accordance with Lazarus and Folkman (1984), it may be difficult to tease out which is problem- and emotion-focused. For example, a nurse experiencing moral distress may become more emotionally invested in a particular clinical situation, but if this is done in order to provide care that is compassionate, then she has also used this integration of emotion into her care to solve the problem of making the patient just some rational, objective response to treatments. Thus, the emotion-focused coping may have a way of also solving problems effectively and thus it is difficult to tease out the two as Lazarus and Folkman (1984) said in regards to a blending of the two styles in moments such as warfare or high stress. Lievrouw et al. (2016) noted that doctors and nurses changed their ways of managing moral distress depending on personal or work-related experiences, and perceived team culture. However, this study only explored adult SICU nurses, but it did not discount that some other ways of managing moral distress may not be dependent on the profession, but also the type of clinical situation and the context in which it occurred.

Several constructive and maladaptive coping behaviors are described in the literature. According to Bruce et al. (2015), constructive behaviors included venting, mentoring, and building team cohesion while maladaptive behaviors included pas-dedeux (incorporating disparate clinical perspectives into the content of patient and family conversations, which does not provide a solution and only serves to obfuscate making a treatment decision), fighting (e.g., verbal confrontation), the use of combat language such as "battle", and desensitization. Findings from Bruce et al. (2015) maintained that healthcare professionals might withdraw or detach from patient care after incessantly feeling unable to protect professional integrity. Importantly, in Bruce et al.'s (2015) study, surgical ICU healthcare professionals experienced more unrelieved moral distress 
than the medical ICU, which might be a result of unit culture and differences in professional backgrounds. It may also have to do with the amount and type of patients they treat, which this study explored. They denied gender differences as the culprit for differences between surgical and medical ICU coping behaviors, but their participants were mainly male physicians and female ancillary staff — consequently no male nurses, which is a limitation and warrants further exploration. Thus, this study recruited male nurses in order to create a heterogeneous sample representative of the SICUs under study.

This study explored coping efforts, which may be emotion- or problem-focused, and/or some combination of both—not limited to one surgical ICU (where more maladaptive coping mechanisms were noted) like Bruce et al. (2015) or gender-biased to only females like Lievrouw et al.'s (2016) study. Johnson-Coyle et al.'s (2016) study took place in one ICU and used quantitative, cross-sectional methods that did not explore how age, sex, marital status, or religiosity may have impacted moral distress. This study did not explore these concepts, except for age and sex because they were a part of the demographic questionnaire discussed later. However, it is important to recognize that since this study was qualitative it is difficult to predict what perceptions and experiences were discussed with individual nurse participants because the data was contingent upon their firsthand accounts and was thus generated by these nurse participants. This study mitigated the other studies' limitations by sampling multiple nurses of diverse age and experience, including males, across two surgical ICUs.

\section{Significance}

Addressing moral distress and moral residue has clinical significance because the negative consequences affect ICU nurses' ability to practice and influence the quality of 
patient care. Some hypothesized negative consequences include burnout, withdrawal and detachment from the ethical dimensions of practice, conscientious objection, quitting a job, or quitting nursing altogether (Epstein \& Hamric, 2009). According to Corley (1995), $12 \%$ of ICU nurses $(n=111)$ reported that they left a position due to moral distress. Hamric and Blackhall (2007) noted that $45 \%$ of ICU nurses $(n=90)$ that completed a shortened form of the MDS (Corley, Elswick, Gorman, \& Clor, 2001) designed specifically for ICU practice with patients at the end-of-life stated they left or considered leaving a nursing position due to moral distress. Hamric et al. (2012) corroborated these findings with the Moral Distress Scale-Revised (MDS-R), revealing that $31 \%$ of ICU nurses left a prior position or considered leaving their current job because of moral distress. These results are significant because they demonstrate the negative impact of moral distress and moral residue on nurses in the high stress ICU. The significance of addressing moral distress is not only to make practice more ethical, leading to improved patient outcomes and perceptions of care, but also to promote retention and professional development of nurses in dealing with moral issues in practice in the ICU environment.

Acknowledging and validating experiences of moral distress and moral residue brings a sense of relief to nurses (Epstein \& Hamric, 2009). Alternatively, listening to these experiences will not prevent moral distress, because nurses are forced to practice in a way that continually violates their sense of moral integrity. However, exploring perceptions and experiences through a qualitative descriptive approach will lay the groundwork for a future research trajectory that will seek to address the crescendo effect by developing interventions aimed at preventing unresolved moral distress and moral 
residue among ICU nurses. This research will identify the moral issues that are of primary concern to ICU nurses. In addition, data from this study will seek to corroborate that morally distressing experiences negatively influence the quality of patient care and the potential harm to patients as identified by the focus groups in Henrich et al.'s (2017) study. Addressing the crescendo is multifaceted across various levels within an organization with respect to understanding how frequency and intensity impact the individual experience and at various levels in the clinical environment: patient/individual, unit/team, and system/organization levels.

Importantly, Epstein and Hamric (2009) clarified that morally distressing experiences occur because poor unit/team performance prevents interdisciplinary communication and collaboration, and healthcare organizations lack policies and leadership that acknowledge and validate moral distress and moral residue among nurses. This study was significant, because it explored moral distress, moral residue, stress and coping, and perceptions of quality patient care among adult SICU nurses in two SICUs. However, individual-level experiences of moral distress are situated within a broader context, thus it is important to acknowledge the existence of moral distress outside the realm of the individual nurse's experience to illustrate how systemic the phenomenon is in healthcare. Unless addressed, moral distress and moral residue will continue to result in unsafe practice, because the moral issues are not being resolved. Healthcare systems and the hierarchies that operate within them-such as a physician giving orders to a nurse to continue treating a patient near the end-of-life with a poor prognosis_-exploit nursephysician relationships through power differentials. Experiences of moral distress and moral residue are not acknowledged through a standardized approach, which is damaging 
to nurses personally and professionally (Epstein \& Hamric, 2009). For example, the patient pays the ultimate price, because they are in effect tortured at the end-of-life through aggressive therapies that the nurse feels they are unable to mitigate. Thus, the significance of this study is to examine moral distress and moral residue in such a way that future individual, unit, and system level intervention research may understand how moral distress first manifests and is managed by adult SICU nurses. This research is of significant importance because this population has demonstrated a lack of constructive coping with moral distress in previous research by Bruce et al. (2015).

A potential moral critique of healthcare systems, organizations, and hospitals is that the burnout and turnover of nurses indirectly transfers into poor quality of care for patients. The findings from this study may be used as groundwork to develop a measure of moral residue and serve as a source for interventions that address and mitigate moral distress and moral residue. Results from this study may help create policies and procedures that allow nurses to safely access morally reflective spaces and opportunities for de-briefings. Researchers, educators, administrators, and clinicians will be able to identify, recognize, and develop interventions to alleviate the negative impact on nurses and the quality of care for patients, by better understanding how the concept of moral distress and moral residue manifests in nurses.

The central problem this study investigated was root causes of moral distress by exploring nurses' perceptions and experiences of moral distress and moral residue in the adult SICU environment and the linkage between moral distress, moral residue, coping, and perceived quality of patient care. Moral distress and moral residue negatively impact nurses because of the challenge to their values, and this has implications for the quality of 
patient care and the main obligation of a nurse: to be a patient advocate. Because individuals cope differently with stress and exhibit different ways of managing their moral distress and residue, the need for this study was highlighted by the urgency to explore how these diverse manifestations influenced not only their personal well-being but also their perceived quality of patient care.

This study explored the perceptions and experiences of moral distress and moral residue among individual SICU nurses. The aim was to describe and interpret their firsthand accounts through “ $\ldots$ a comprehensive summary of an event in the everyday terms of those events" (Sandelowski, 2000, p. 336), using a descriptive qualitative approach. If nurses' experiences of moral distress are a symptom of moral issues occurring at the bedside, such as futile and aggressive treatment at the end-of-life, then nurses' experiences should be acknowledged before harm is unknowingly perpetuated. This method of qualitative descriptive research will reveal root causes of moral distress by seeking to identify the moral issue(s) that create negative experiences and/or powerlessness for nurses.

Moral distress is a challenge to core obligations and values (Epstein \& Hamric, 2009), including challenges, threats, and violations to personal and professional moral integrity (Thomas \& McCullough, 2015). The core nature of the nurse's values and integrity are called into question as a result of internal constraints (e.g., lack of courage, inexperience) and external constraints (e.g., orders, policies), which are perpetuated by broader healthcare systems at the unit/team and organizational levels (Dudzinski, 2016; Hamric, 2014; Thomas \& McCullough, 2015; Varcoe, Pauly, Webster, \& Storch, 2012). Although this current study only focused on individual SICU nurses, qualitative 
interviews also illuminated unit and system-wide problems in the continuum of care that influenced the individual nurse participants' perceptions and experiences of moral distress and moral residue, particularly as they applied to the frequency and intensity of morally distressing experiences.

\section{Specific Aims}

The specific aims of this study were designed to:

Aim 1. Explore the perceptions and experiences of moral distress and moral residue in adult SICU nurses.

Aim 2. Explore the antecedents and consequences of moral distress and moral residue in adult SICU nurses and the influence on perceived quality of care.

RQ2.a. What moral issues create moral distress and moral residue in SICU nurses?

RQ2.b. How does moral distress and moral residue impact their perceived quality of care for adult patients in the SICU?

RQ2.c. How does moral distress and moral residue influence professional practice standards, personal moral integrity, and values among SICU nurses?

Aim 3. Determine the root causes that contribute to a crescendo effect of moral distress with respect to time (e.g., years of exposure), frequency (e.g., how often in their nursing career), and intensity (e.g., severity of the experience) of exposure in the SICU nurses.

Aim 4. Describe nurses' professional and personal coping methods and behaviors related to moral distress and subsequent moral residue in the SICU to determine if they are temporally, contextually, and/or situationally-specific to particular root causes of moral distress. 


\section{CHAPTER 2 REVIEW OF LITERATURE}

\section{Background - Moral Distress}

This review of literature was organized conceptually in order to present an amalgamation of concepts that relate to moral distress, moral residue, stress and coping, and quality of patient care. Importantly, this review of literature is an exploration into how moral distress has developed in the ICU. Furthermore, this review of literature investigates how conceptual ambiguity has led to a lack of clarity into the negativity of moral distress. Moreover, by exploring various related concepts to moral distress, this review of literature highlights the importance of acknowledging and addressing moral distress because of its implications for the delivery of ethical healthcare. Thus, this review of literature will proceed with an investigation of the subsequent concepts: moral distress; conceptual ambiguity of moral distress; healthcare rationing; quality of life, effectiveness of therapies, and cost; hospital organizational ethical climate and the ethical dimensions of practice; futile treatment in the ICU; moral distress at the end-of-life; moral distress and conscientious objection; moral distress and burnout; and identified gaps in the literature.

\section{Moral Distress}

Moral distress occurs in clinical situations where life is prolonged in dying patients, harm is caused to patients, there is an inability to reduce a patient's pain and

suffering, and a general dehumanization of patients, which challenged a nurse's personal 
moral integrity (Dudzinski, 2016; Hamric et al., 2012; McCarthy \& Gastmans, 2015; Wilkinson, 1987). These clinical situations are just some of the causes of individual-level experiences of moral distress. For example, Hamric and Blackhall (2007) noted unit-level causes. These researchers reported that nurses experienced less collaboration than physicians and a perception of a negative ethical environment. The nurses were less satisfied than physicians with the quality of care provided on their units. Assessments of quality patient care were related to perceptions of collaboration, and it was noted that explicit conversations about moral distress, recognition of different nurse/physician values, and improving collaboration are all ways to improve the ethical climate at the unit-level in ICUs and may decrease frustration related to differing perspectives between nurses and physicians (Hamric \& Blackhall, 2007). Epstein and Hamric (2009) developed a model that demonstrated how increasing moral residue over time resulted in the crescendo effect. Importantly, the root causes that lead to the crescendo effect are not just morally distressing clinical situations. Rather, according to Hamric (2012), moral distress is a result of unit and system factors such as recurrent unit cultural and system factors that include poor healthcare team communication or lack of administrative support. Thus, moral distress may manifest as a myriad of symptoms and feelings, ranging from rage to anxiety and sadness as a nurse's moral agency is challenged (Corley, 2002; Jameton, 1993; McCarthy \& Gastmans, 2015).

Andrew Jameton (1984) initially defined moral distress as knowing the right course of action to take, but being hindered because of institutional constraints. However, this definition is vague. Alternatively, Webster and Baylis (2000) defined moral distress as arising when one fails to do what they believe to be the right action (or fails to do so to 
one's satisfaction) for one or more of the following explanations: a judgment error, a personal failing (e.g., a character weakness such as systemic avoidance), or some circumstance beyond one's control. This definition will be used in this study, because it adequately accounted for aspects of moral distress that will be explored, such as individual perceptions and experiences of moral distress, moral residue, stress and coping, and quality of patient care.

The symptoms of moral distress illuminate the negative consequences for nurses. Sources confirmed that moral distress is characterized by frustration, guilt, physical symptoms, and anxiety related to repressed or violated moral values and beliefs (Austin, Lemermeyer, Goldberg, Bergum, \& Johnson, 2005; Gutierrez, 2005). It is important to make a distinction between moral distress and the psychological stress that it imposes. For example, moral distress leaves a nurse feeling powerless to effect change in the perceived moral atrocities being committed at the bedside and this moral distress creates psychological distress due to feelings of powerlessness and conflicting loyalties. Moral distress is differentiated from concepts such as emotional distress, compassion fatigue, and posttraumatic stress disorder, because these latter three concepts lack a moral component where core values, integrity, and obligations are challenged (Epstein \& Hamric, 2009). The nurse knows the moral action to take in morally distressing critical events, but the ability to act is limited by the healthcare hierarchy and barriers in the clinical culture, such as an environment where the discussion of the ethical dimensions of practice are not encouraged or addressed.

The moral component is moral because there is a challenge to personal moral integrity (Webster \& Baylis, 2000). Integrity necessitates that the individual speaks and 
acts consistently with their deeply held values, beliefs, and principles; however, if an action the nurse knows is right is prevented from occurring, then their personal moral integrity is called into question, resulting in moral distress (Webster \& Baylis, 2000). Identifying moral issues that cause moral distress is pivotal to improving patient care, because the experience reflects the moral convictions and personal moral integrity of the nurse, since the nurse knows the right thing to do and has clarity about their own values (Dudzinski, 2016). Moral distress experienced by nurses has the following essential attributes: (1) internal constraints; (2) external constraints; (3) a clinical situation; and (4) negative feelings, powerlessness, conflicting loyalties, and uncertainty due to the first three characteristics (Dudzinski, 2016; Hamric et al., 2012; McCarthy \& Gastmans, 2015; Russell, 2012; Savel \& Munro, 2015).

Moral distress may cause a disconnection from personal values and beliefs, because of threatened moral integrity (Hanna, 2004). Powerlessness to act and self-doubt are internal constraints of moral distress. External constraints include staffing shortages, healthcare hierarchies, and nurses' absence in decision-making (Hamric et al., 2012). These constraints create internal psychological disequilibrium and negative feelings associated with challenging personal values (Wilkinson, 1987). Moral distress occurs when a judgment error, personal failure, or external situation prevents the right course of action (Webster \& Baylis, 2000). Thus, the negative sequela from moral distress highlight the importance of acknowledging the damaging effects of this phenomenon for nurses, other healthcare professionals, patients, units, and healthcare systems.

Numerous examples are evident in the literature to explore, define, describe, and quantify moral distress. The importance of this phenomenon is evident in the perceived 
immoralities occurring in patient care, because nurses' perceptions and experiences provide insight into the ethical dimensions of practice. Nursing's goal is to study the whole person and highlight unique differences in the caring process (Earle, 2010). The development of nursing knowledge is predicated upon understanding human experiences in their contexts (Rodgers, 2005). Moral distress is a fluid concept that has temporal and contextual influences because the meaning changes in different settings (Russell, 2012).

The Moral Distress Scale-Revised (MDS-R) (Hamric et al., 2012) was developed from the original Moral Distress Scale (MDS) for ICU nurses (Corley et al., 2001) to identify the frequency and intensity of moral distress in ICU as well as non-ICU settings and within multiple disciplines by looking at root causes (Hamric et al., 2012). Importantly, the MDS-R was tested in the ICU settings first. Using the MDS and MDS$\mathrm{R}$, researchers have found that moral distress has been correlated with multiple factors. Researchers have found a positive association with age (MDS) (Browning, 2013; Shoorideh, Ashktorab, Yaghmaei, \& Alavi Majd, 2015), work experience (MDS-R) (Hamric et al., 2012), and gender, with females experiencing more distress than males (MDS-R) (O'Connell, 2015). Moral distress is negatively associated with ethical climate (Hamric et al., 2012), and positively associated with nurses' intent on leaving their jobs using the MDS-R (Hamric et al., 2012). An ethical climate is a supportive environment where nurses feel comfortable identifying and discussing moral issues in clinical practice (Whitehead et al., 2015). A unit has a low ethical climate if the environment is not supportive and the staff feel unable to bring up and discuss moral concerns.

Despite available moral distress research, these quantitative studies fail to recognize the importance of the nurse's individual experience of moral distress and the 
implications for building moral residue with subsequent pile-up and the crescendo effect, coping, and perceptions of quality patient care. For example, the existence of moral distress in the critical care environment has been demonstrated from research; however, the implications of the phenomenon for healthcare hinges on the morality of patient care. If nurses repeatedly feel negative and powerless about their care in the ICU, then their experiences surrounding moral distress should be explored as well as the moral issue negatively impacting ICU nurses and the patients for whom they provide care.

Phenomenological end-of-life moral distress research reveals valuable insights into the lived experience. For example, Robinson and Stinson (2016) used a phenomenological qualitative design to interview a convenience sample of eight nurses, all of whom reported experiencing moral distress. A prevailing theme was ... asking God for forgiveness, because "Nurses often expressed regret for the care they felt forced to render to patients and felt the need to be forgiven" (p. 238). Central to the experience of moral distress is the nurse's powerlessness to change his or her response to a moral wrong because of pressure from internal and external constraints, particular clinical situations, and the expectation by institutional hierarchy to comply with the morally distressing action.

Choe, Kang, and Park (2015) used Giorgi’s phenomenological approach to explore perceptions of morally distressed ICU nurses $(\mathrm{N}=14)$ caring for adult patients identifying five themes: ambivalence towards treatment and care, suffering resulting from a lack of ethical sensitivity, dilemmas resulting from nurses' limited autonomy in treatments, conflicts with physicians, and conflicts with institutional policy. Epstein (2008) used hermeneutical phenomenology to explore the lived experiences of nurses (n 
$=21)$ and physicians $(\mathrm{n}=11)$ after infant deaths in the neonatal ICU. The main theme was: create the best possible experience for parents. Importantly, this overarching theme was accompanied by several subthemes that helped accomplish this goal for parents, such as: building relationships, preparing for end-of-life, and creating memories. Additional themes outside of this best experience for parents applied to providers' personal experiences and included: moral distress, parent readiness, and consent for autopsy. Moreover, the time of interest was once the decision was made to withdraw care all the way to the completion of postmortem care. This period of time was designated as the end-of-life period. However, healthcare providers' experiences were found to be deeply influenced by the time immediately before the end-of-life period, particularly the span of time that involved decision-making. Thus, not just the end-of-life period, but also the time where treatment was still being provided and the phase of subsequent decisionmaking regarding the patient's care deeply influenced providers' experiences. According to Epstein (2008), all instances of moral distress were a result of prolonged, aggressive treatment that was perceived to be futile.

Manara, Villa, and Moranda (2014) used a phenomenological-hermeneutic case study approach to determine the processes leading to moral distress for a nurse $(\mathrm{N}=1)$. How a nurse interprets a particular situation has an effect on them and influences their decisions and behaviors (Manara et al., 2014). In addition, the integration between logical-formal thinking (scientific thinking) and narrative thinking (attention to the entire situation that does not depersonalize participants, but incorporates respect) enables a nurse to not just consider the patient and their diagnosis, because they will also consider the person as someone who thinks, feels, and experiences (Manara et al., 2014). 
Ultimately, the best solution is one that considers the decision-making power of the patient and the nurse, while both are also aware of the values involved (Manara et al., 2014). Importantly, implications for future nursing research necessitates an investigation of how feelings created by emotional acceptance of the event influences ethical decisionmaking and moral distress.

Moral distress is not just exclusive to clinical situations of futile treatment at or near the end-of-life. For example, Hamric et al. (2012) took note of other situations that result in moral distress: (a) inadequate or inappropriate informed consent; (b) working with other caregivers that are not as competent as the care requires; (c) lack of continuity of care; (d) providing care not in the patient's best interests; (e) inadequate pain relief; (f) hastening the dying process; and (g) disregard for patients' wishes. Another study by Woods, Rodgers, Towers, and La Grow (2015), findings from 415 nurses that answered the MDS-R revealed that the most frequent situations that they identified as causing moral distress were: (a) providing less than optimal care due to management decisions; (b) witnessing patient care quality suffer due to lack of provider continuity; and (c) working with other healthcare workers who are less competent. Furthermore, the most morally distressing experiences were a result of: (a) working with others who are unsafe or incompetent; (b) witnessing poor quality of care due to poor communication; and (c) seeing patients suffer to due to lack of provider continuity. Interestingly, these experiences of moral distress were highlighted by situations where the nurse felt that they were unable to provide care that they was appropriate and in the patient's best interests. Thus, situated within this experience of moral distress is the concern for the patient's quality of care. 
Oncology nurses are more likely to experience moral distress as evidenced by the patient population for whom they provide care. Oncology nurses completed the MDS-R questionnaire developed by Hamric et al. (2012) and results revealed that the two highest scoring items for moral distress frequency were: ignore situations in which patients have not been given adequate information to ensure informed consent and carry out the physician's order for what the nurse considers to be unnecessary tests and treatments (Ameri, Safavibayatneed, \& Kavousi, 2016). Regarding moral distress intensity, the two highest scoring items were: work with nurses or other healthcare providers who are not as competent as the patient care requires, and provide care that does not relieve the patient's suffering because the physician fears that increasing the dose of pain medication will cause death (Ameri et al., 2016; Hamric et al., 2012). These items reveal that providers experience moral distress as a result of their inability to be a patient advocate, which is no fault of their own but rather a result of the context in which they are situated to work.

Moral distress affects multiple healthcare professionals (e.g., nurses, physicians, pharmacists, social workers, case managers, respiratory therapists), thus healthcare organizations should provide resources to decrease moral distress by focusing greater attention on the context of ethical dilemmas that occur in practice (Kälvemark, Höglund, Hansson, Westerholm, \& Arnetz, 2004). Improving the ethical environment in the ICU may be achieved through open discussions of moral distress, recognition of value differences between nurses and doctors, and focusing on improved collaboration between nurses and doctors, which may decrease conflict resulting from varying perspectives, thereby improving the quality of patient care (Hamric \& Blackhall, 2007). In a study using a questionnaire developed from focus groups with physicians and nurses, patients 
assessed as receiving futile treatment by both physicians and nurses together were more likely to die in the hospital than patients assessed solely by the nurse (76\% versus $32 \%, \mathrm{p}$ $<.001)$ or the physician $(76 \%$ versus $57 \%, \mathrm{p}=.04)$ (Neville et al., 2015). Interestingly, physicians are responsible for writing orders for life-sustaining treatment and its withdrawal, thus their assessments of futile treatment alone resulted in a higher mortality rate for patients. However, nurses' assessments alone resulted in a comparatively low mortality rate for patients perceived to be receiving futile care. This may be because nurses define futile treatment differently than physicians, because of their role differences and also because nurses focus on factors such as suffering rather than survival (Neville et al., 2015). These results suggest the value of collaborative decision-making between physicians and nurses at the end-of-life (Neville et al., 2015), which may help to mitigate moral distress associated with aggressive treatment at the end-of-life and futile treatment because ethical discussions regarding moral issues occur. Also, it is important for the nurse, who has more patient contact than any other healthcare professional, to collaborate and communicate with other healthcare team members and the patient's family. Through this transparent sharing of information, the team may arrive at a joint consensus regarding the patient's treatment plans and goals that is not coercive or manipulative, but mirrors the wishes and preferences of the patient who is perhaps incapacitated.

\section{Conceptual Ambiguity of Moral Distress}

The significance of addressing the specific aims is necessary to combat misconceptions about moral distress. Webster and Baylis' (2000) definition of moral distress states that nurses cannot do what they feel is right. Jameton's (1984) definition of moral distress is adequate, but it should be more specific in order to clearly delineate 
what is at stake in an experience of moral distress. At stake is a moral issue that is perceived to be wrong and damaging to the patient, which includes nurses' personal and professional moral integrity in order to encompass their core duties and obligations. It is the inclusion of nurses' challenged moral integrity that necessitates a moral evaluation of the clinical situation, because a perceived immorality is occurring in the delivery of patient care. This implies that harm is about to be done to a patient or an outcome that negatively influences their trajectory in the hospital. Webster and Baylis's (2000) definition of moral distress encompasses the internal and external constraints that characterize the moral issue(s) of the experience. For example, an error of judgment and some personal failing reflects the internal constraints such as lack of courage or experience, which leads to feelings of remorse and guilt (Dudzinski, 2016).

Moreover, nurses are constrained from enacting their own moral conviction externally, when a decision is made by an outside source (e.g., institutional hierarchy), such as a physician's order, organizational policies, or family's wishes. Importantly, the nurse may cope with and mitigate these occurrences of moral distress through personal therapeutic responses such as, exercise and socializing with friends after work. While it is important to rationalize and reconcile what occurs at work so they may come back the next day and work and be productive, it says nothing about fixing the moral issue or even attempting to address it. Thus, coping is important, but it will not fix the underpinning moral issue. Similarly, it is important to determine how the nurse identifies actions that will directly help the patient and improve their quality of care and subsequent outcome. A core implication of the experience of moral distress requires identification and action to resolve the underlying moral issue(s), otherwise moral distress will continue despite how 
a nurse copes with its everyday occurrence. For example, a nurse may go home after every shift and consume alcohol, exercise, or socialize, but the moral distress occurring at work will still be there when they return, regardless of how they previously coped with the situation.

Moral distress affects professional practice standards, personal moral integrity, values, and well-being, because of the perceived immorality of an action (Epstein \& Hurst, 2017). Moral distress is characterized by an experience where the nurse knows the right thing to do. For example, restraining a patient may be distressing, but it is morally distressing when the nurse believes it is wrong and they bear the moral responsibility. Nurses' experiences may not include an underlying moral issue at stake. According to Dudzinski (2016), a nurse suffering from moral distress may not be correct. This does not mean that a nurse's moral intuitions should be ignored. However, nurses' intuitions, just like humans' intuitions, are not infallible and should still come under ethical inquiry. Moreover, clinical practice in the ICU is complex, so when multiple disciplines are working together, the potential for disagreements is pervasive, which necessitates that any accusations of immorality or wrongdoing come under meticulous ethical scrutiny. In a clinical context, the clarification of ethical issues through deliberate communication, collaboration, and education within the interdisciplinary team enables healthcare professionals to understand their responsibilities and the responsibilities of others, while also preventing decision-making in isolation (Hamric, 2014). When a nurse experiences moral distress, their perceived immorality may be inaccurate; however, the harm to the nurse is through the perceived harm they feel enacted towards the patient. In other words, the nurse will feel emotionally distressed even if an "objective" analysis 
reveals that there is no moral issue at stake. However, distress is still felt in this experience. Thus, it is important to distinguish moral distress from emotional distress. A nurse who does not perceive a moral issue at stake and while being compromised is not experiencing moral distress. Moral distress is always emotionally distressing and this is a hallmark in identifying the phenomenon. According to Hamric (2014), when a nurse's moral integrity is compromised by a morally distressing clinical situation it results in emotionally distressing responses, which includes avoidance, frustration, anger, guilt, and physical symptoms. Moreover, moral distress is emotionally distressing, just like burnout, compassion fatigue, and posttraumatic stress disorder; however, moral distress contains a threat to one's view of what is right and wrong, which these other concepts do not contain (Hamric, 2014). For example, moral distress is characterized by an experience of having one's moral values or obligations compromised, or allowing them to be compromised (Hamric, 2014). Thus, moral distress lays bare one's view of their self as a moral professional and moral person in a profession where critically ill patients are cared for in a state of vulnerability (Hamric, 2014). Potential consequences of unaddressed moral distress in particular types of clinical situations that impact the nurse's moral integrity include turnover and burnout from the high stress ICU healthcare environment (Epstein \& Hamric, 2009; Hamric et al., 2012).

Based on this analysis, it is important to distinguish in a definition of moral distress a compromise to one's moral integrity, or allowing one's moral integrity to be compromised. This is what makes moral distress distressing: the blatant challenge to one's view of what is right through an assault or challenge to one's moral integrity. A definition should be broad enough to include a varied response to the moral distress 
experience, but still retain this focus on emotional distress as a result of challenges to one's view of what is morally "right" and also their values, obligations, and moral integrity. This study will endorse Webster and Baylis' (2000) definition of moral distress: as arising when one fails to do what they believe to be the right action (or fails to do so to one's satisfaction) for one or more of the following explanations: a judgment error, a personal failing (e.g., a character weakness such as systemic avoidance), or some circumstance beyond one's control. According to Epstein et al. (2016), the definition of moral distress identifies that nurses take moral responsibility when patients are not receiving care that is appropriate, effective, or safe, even when forces outside their control prevent remedying the situation (e.g., a physician's orders, institutional hierarchy such as a policy or a management decision, and/or a family's treatment directives for their incapacitated patient's treatment plan). However, Webster and Baylis's (2000) definition of moral distress describes nuance in what is the perception of wrongful duty experiences and was thus used instead of Epstein et al.'s (2016) definition.

The actual manifestation of an individual's emotional response to moral distress may or may not be commensurate with the underlying moral issue(s) at stake, meaning that everyone has a particular way of addressing their own emotional distress, but the focus must emphasize that repeated exposure to morally distressing events in the ICU results in a degradation of one's moral integrity, which leads to moral residue that affects practice either indirectly (e.g., through de-sensitization) or directly (e.g., through the powerlessness and inability to act in the patient's best interest). This study sought to determine how SICU nurse's perceptions and experiences of moral distress and moral residue not only impacted their stress and coping, but also their quality of care. 
A broader definition of moral distress proposed the inclusion of moral uncertainty, mild distress, delayed distress, moral dilemma, bad moral luck, and distress by association (Campbell, Ulrich, \& Grady, 2016). Earlier it was suggested that a broader definition of moral distress include the perception of a moral wrong, because of moral issues occurring in practice that prevented the nurse from "doing the right thing". However, it was not meant to suggest that it dilute the concept by including other concepts that were not morally wrong. Campbell et al. (2016) suggested that moral distress is "one or more negative self-directed emotions or attitudes that arise in response to one's perceived involvement in a situation that one perceives to be morally undesirable" (p. 6). Morally undesirable cases include difficult choices, unfortunate outcomes, or inherent risks of healthcare categorized as moral problems, which ignores the central defining feature of moral distress, namely the threat to one's moral integrity, because of an underlying moral issue that prevents the nurse from doing what they believe to be right (Epstein et al., 2016). The threat to one's moral integrity can be personal, professional, or both (Thomas \& McCullough, 2015). Certainly diverse values, cultural norms, personal viewpoints, and upbringings all serve to influence one's moral outlook, but should not obfuscate the negativity of moral distress. Lack of conceptual clarity has been counterproductive to research (McCarthy \& Deady, 2008). Moreover, Dudzinski (2016) created a table of definitions from a review of literature to convey a multitude of conceptual definitions for moral distress, which exemplifies the ambiguity in defining moral distress (see Table 1).

Table 1

Definitions of Moral Distress 
When one knows the right thing to do, but institutional Jameton, 1984 constraints make it nearly impossible to pursue the right course of action

When the practitioner feels certain of the ethical course of action Hamric \& but is constrained from taking that action Blackhall, 2007

The painful psychological disequilibrium that results from recognising the ethically appropriate action, yet not taking it, because of obstacles such as lack of time, supervisory reluctance, an inhibiting medical power structure, institution policy or legal considerations

You act in a manner contrary to your personal and professional values, which undermines your integrity and authenticity

American Association of Critical Care Nurses (AACN), n.d.

The pain or anguish affecting the mind, body or relationships in response to a situation in which the person is aware of a moral Rushton, Kaszniak, \& Halifax, 2013 problem, acknowledges moral responsibility and makes a moral judgement about the correct action; yet, as a result of real or perceived constraints, participates in a perceived moral wrongdoing

Moral distress occurs when situational constraints prevent a Wilkinson, 1989 nurse from implementing a moral decision s/he has made. . . For moral distress to occur, a case must arise in which the nurse recognises a moral issue and believes she or he is responsible for her or his own actions in the situation

The suffering experienced as a result of situations in which Mitton, Peacock, individuals feel morally responsible and have determined the Storch, Smith, \& ethically right action to take, yet owing to constraints (real or 
perceived) cannot carry out this action, thus believing that they are committing a moral offence

Traditional negative stress symptoms that occur due to situations Kälvemark et al., that involve ethical dimensions and where the healthcare 2004

provider feels she/he is not able to preserve all interests and values at stake

Moral stress is experienced when nurses are aware of what Lützén, Cronqvist, ethical principles are at stake in a specific situation and external factors prevent them from making a decision that would reduce Magnusson, \& Andersson, 2003 the conflict between the contradicting principles

Reprinted from "Navigating moral distress using the moral distress map," by D. M. Dudzinski, 2016, Journal of Medical Ethics, 42(5), p. 322.

There are more similarities in the above definitions than differences, because the key is a focus on what the healthcare professional believes to be right is being challenged and called into question. According to Campbell et al. (2016), moral distress only arises when a person thinks they know the morally correct course of action, but they also stated that sometimes we fall short of knowing what to do or having particular ethic's knowledge. Thus, Campbell et al. (2016) makes the point that it is not always easy to know the right course of action to take, particularly with the abundance of moral theories and principles. According to Campbell et al. (2016), there is a vast array of potential actions in any particular clinical situation; therefore, it is impossible to predict all the consequences. But the challenge occurs when we start to question nurses' ability to determine what is and what is not ethical. For example, if the vast amount of moral distress literature occurs in situations at the end-of-life, because those clinical situations are difficult with regard to conditions like medical futility, inadequate communication, and poor ethical climate, then it is important to listen to nurses when they feel distressed 
about how these situations reconcile or lack thereof. Thus, by broadening a definition of moral distress like Campbell et al. (2016) suggests, then we run the risk of taking away the focus from the challenges to one's moral integrity and values, which is what the experience of moral distress is about and why it is so damaging to healthcare professionals. Thus, there are numerous definitions of moral distress in the literature, but the key is to not dilute the concept by taking away from the focus on challenges to moral integrity. Just because nurses may not know about ethical theory or all the particulars of a case does not mean their assessment and evaluation is wrong. According to Epstein et al. (2016), broadening an already narrowly defined concept, such as moral distress, does not offer any benefits and in fact weakens the arguments for further study. Specifically, moral distress cannot mean whatever the researchers writing about it have taken it to mean, instead it needs to focus on compromises to moral integrity, values, and obligations; otherwise, broadening a concept to include moral uncertainty only serves to dilute the focus on making patient care more ethical (Epstein et al., 2016).

\section{Healthcare Rationing, Quality of Life, Effectiveness of Therapies, and Cost}

In this section and also in the subsequent one on quality of life, effectiveness of therapies, and cost, Leonard Fleck's (2009) book, Just Caring, pertaining to healthcare rationing and his theory of rational democratic deliberation were analyzed to provide a meta-view of important healthcare considerations that inform moral distress research. His theory provides insight from a broader view of how decisions are made by healthcare administrative teams and how moral issues relate to financial decisions, which ultimately influence the moral distress nurses experience. For example, decisions to continue treatment that may not benefit the patient are costly and these same resources could be 
used to treat patients who have greater chances of survival. Fleck (2009) suggests that hospitals control costs, through rationing decisions related to cost, budgets, and patients' choices pertaining to their autonomous rights.

Fleck (2009) reinforced the notion that rationing decisions in healthcare are rarely clean-cut and without conflict due to diverse contexts, emphasizing that just and moral claims to limited resources must not deprive others of their rights and access to similar therapies (e.g., renal dialysis). Healthcare needs are infinitely complex and heterogeneous (Fleck, 2009), which makes adjudicating equitable rationing at the federal, state, and organizational-levels difficult. Specifically, age, genetics, multiple co-morbidities, varying socioeconomic statuses, and varying degrees of healthcare usage are just some of the factors that make determining equitable, just, and moral rationing of limited resources enigmatic. Fleck's (2009) key premise emphasized that the moral conclusion cannot appeal to a reduced principle of healthcare justice that includes various nuances of utilitarian, libertarian, or egalitarian perspectives. Instead, issues of healthcare rationing and limited resources with unlimited needs demonstrates that society faces a complex amalgamation of problems not easily solved by appealing to ethical principles. Ethical principles do not lead everyone in the same direction, thus it is important in complex ethical discussions of rights, resources, and needs that communication be transparent, respectful, and weigh all potential courses of action and outcome in order to arrive at a decision that is not harmful to the patient, family, and healthcare team.

Issues of healthcare justice imply that immorality occurs. As it applies to ICU nurses treating patients, the focus is on advocating for their best interests. Treatments that prolong life without any consideration of quality are continuing to increase in scope and 
expenditures, so it is impossible for physicians and other healthcare providers to completely remove themselves from the moral responsibility of healthcare rationing decisions (Fleck, 2009). Moreover, cost and rationing are inextricably linked to moral distress and moral residue, because an inappropriate use of healthcare resources that does not result in any foreseeable change in quality or positive prognosis is simply wasted. Advances in healthcare technology perpetuate moral distress and moral residue, because as individuals age, the ability to keep them alive longer increases, while simultaneously their quality of life diminishes. This situation creates the context for moral dilemmas and moral distress with respect to healthcare rationing, because if the therapies are not creating positive benefits for the patient, then they ought to be stopped or those options at least discussed with the healthcare team, family, and patient.

Applying the principle of justice or respect for autonomy alone does not help physicians make equitable and moral decisions for their patients. In particular, the healthcare system's morality is complex. It is difficult to make confident, isolated decisions that are just and moral decisions in a system that is so complex, particularly since technological advancements make the determination of quality of life difficult and unclear. Collaboration, communication, and education are needed to inform this challenging and complicated context. When the infliction is through one's profession it presents a particularly troublesome problem. The moral distress that some nurses experience creates a canary in the coalmine effect whereupon red flags include turnover, burnout, and resignation (Varcoe et al., 2012). Nurses may still feel as though they are culpable of harm even when their professional obligation to advocate for the patient's best wishes, per the beneficence principle, mandates the continuation of treatment in a 
dying patient. This type of care may be deemed futile, non-beneficial, and aggressive, but the nurse is morally distressed, because they can do nothing to change the course of action due to their limited voice. This is a ragged edge, meaning that it is not a clear-cut solution, and rationing decisions may be made in this context. In particular, responsibility to begin a compassionate conversation with the family about withdrawing treatment while also advocating for limited healthcare resources that may be extended to others in need of that bed is a fine line to balance.

Fleck (2009) asks if it morally permissible to consider the quality of life of a patient that is saved or sustained as being morally relevant to determining whether or not that patient has a just claim to limited healthcare resources. Overall, the interpretation of Fleck leads one to believe that quality of life matters in determining a just allocation of healthcare resource. Quality of life is not unjustly discriminatory when applied to individuals with disabilities, such as those patients suffering from chronic diseases, because of rational democratic deliberation. Rational democratic deliberation is defined as a process of making future healthcare decisions for one's future self in a present moment with other rational human beings, while being ignorant of one's future possible medical conditions and ailments. Thus, in an effort to determine what gets covered by insurance and what does not in a conception of universal healthcare, since it is rational, democratic, and deliberative then it is assumed that all possible options and perspectives were weighed, consensus was sought, and ultimately a decision was made about future healthcare dollars in a way that does not rob others of access to limited resources in an environment of unlimited needs. For example, democratic deliberation is impartial where a conversation is had between un-dominated equals that is honest, transparent, mutually 
respectful, and is committed to giving good reasons as the primary way of arriving at a resolution. Thus, the goal of rational democratic deliberation is to create rules, policies, or practices that everyone can live with because they are fair, reasonable, and not able to be reasonably rejected by participants in the deliberative process. Thus, rationing and prioritization judgments must be made in allocating limited healthcare resources among unlimited healthcare needs. For example, moral and ethical dilemmas are concerned with rationing of healthcare and the exorbitant financial costs inherent in the care of 600-gram premature infants in the neonatal intensive care unit (NICU) with complex healthcare needs, those who need artificial hearts, implantable defibrillators, and left ventricular assist devices for patients with persistent vegetative states, or those in the end-stages of dementia. Fleck (2009) claims that a rational and democratic deliberation is needed whereupon individuals make choices based on potential healthcare needs their future possible selves might require to maintain quality of life.

Specifically, Fleck (2009) advocates for the egalitarian distribution of healthcare resources that is cost-effective, particularly since our current system is bracketed by limited resources and unlimited needs. In this context, it is vital to highlight last chance therapies (particularly salient to the SICU where numerous interventions are attempted to save the lives of patients who undergo traumatic injuries) where thousands of dollars are spent on patients in their last few years, or days, of life with only marginally beneficial outcomes. Rational democratic deliberations regarding healthcare rationing and prioritization of judgments must be influenced by quality of life considerations (Fleck, 2009). For example, if the potential interventions return the patient to "normal life" where they do not need a ventilator and 24-hour nursing care then they may be deemed 
appropriate. But it is a gamble to determine if complex health problems may be meaningfully resolved with medical technologies. If limitless amounts of money are spent on interventions, and the outcome for the patient is limited or poor functioning that also causes undue suffering and harm, then it would seem appropriate for the nurse to feel morally distressed regarding this insufficient and wasteful usage of valuable, limited healthcare resources.

Fleck emphasized that an overall conception of healthcare justice must be governed by two justice-relevant considerations: effectiveness and cost. Specifically, valid and reliable methods of predicting survivors from likely non-survivors and also determining what quality of life a particular patient and their family values. From a justice consideration that seeks to create healthcare access that is affordable and effective for society, the goal is a focus on therapies that do not prolong suffering and result in a livable quality of life free of sustained medical inpatient care. Alternatively, Epstein and Hurst (2017) note, "thank goodness for the keen eye of the attending physician who sees a ray of hope that the team does not see (yet), for this wisdom has certainly saved many a patient's life" (p. 39). It is the case that some patients who do not have a probable chance of survival end up living functional lives. However, it potentially becomes a law of diminishing returns as last chance therapies are used time and again in our current healthcare system to save lives with marginally beneficial results, which ultimately robs healthier individuals who are acutely ill of limited healthcare resources to treat their ailments that have a higher probability of recovery.

Counter to this argument is the idea that human life is priceless, also known as the sanctity of human life. If human life is priceless, then society should prolong all human 
existence, due to a moral obligation. However, Fleck (2009) reiterates the harsh reality that healthcare resources are limited and we should use quality of life as a metric because that is our obligation to justice — even though it may be potentially subjective. The whole crux of Fleck's argument is to ration and allocate limited healthcare resources openly through a fair, cost efficient, and effective sustainment of quality of life. Fleck's argument provides important implications for moral distress and he has provided a persuasive position to advocate for moral and just allocation judgments based on quality of life, because in treating patients in the SICU, quality of life matters, cost matters, effectiveness of therapies matters.

A concern is patient autonomy trumping all other considerations. For example, in instances where a patient's decision to continue treatment is a futile effort, because the outcome of their illness trajectory will still result in death without any return to a functional quality of life post-intervention of any kind. Thus, according to Fleck (2009), individuals should be free to choose their healthcare providers, treatments, and hospitals so long as these choices do not threaten to undermine or violate a shared understanding of healthcare justice. Thus, individuals are able to make any decision they want, so long as their exercise of that right does not undermine or threaten the just claims of others to needed healthcare (Fleck, 2009). Thus, the caveat is that patient autonomy should be exercised, but it should not trump the rights of others and other's ability to access needed healthcare.

In conclusion, Fleck's rational democratic method, healthcare rationing, quality of life, effectiveness of therapies, and cost demonstrated a recurrent meta-theme: problems with healthcare exist and they may cause nurses to feel morally distressed about the care 
they feel forced to provide, or unable to change for the betterment of the patient. Flecks' (2009) rational democratic deliberation, quality, cost, and effectiveness of therapies illustrates the complexity of ethical problems in healthcare that are rarely easily resolved by a singular mandate. Instead, these problems take time, consideration, and focus on the ethical dimensions of practice in order to advocate for patients' best interests.

\section{Hospital Organizational Ethical Climate and the Ethical Dimensions of Practice}

A healthy work environment may have implications for not only the well-being of nurses, but also on the quality of care of the patients receiving treatment. Healthcare is more than a system of delivering new and advanced medical treatments and care that reduces ailments - health is also created where people live and work. Thus an important social determinant of health is an individual's work environment (Mason, Gardner, Outlaw, \& O'Grady, 2016). Nurses are bound by their professional code of ethics to advocate for the safety and well-being of their patients, but often physicians' orders and families perpetuate structures that may challenge one's professional obligations and personal ethical views of what matters morally. According to the American Nurses Association's (ANA)'s (2015) Code of Ethics for Nurses with Interpretive Statements:

Nurses should address concerns about the healthcare environment through appropriate channels and/or regulatory or accrediting bodies. After repeated efforts to bring about change, nurses have a duty to resign from healthcare facilities, agencies, or institutions where there are sustained patterns of violation of patient's rights, where nurses are required to compromise standards of practice or personal integrity, or where the administration is unresponsive to nurses' 
expressions of concern. Following resignation, reasonable efforts to address violations should continue. (p. 24)

Addressing moral distress includes more than nurses' professional obligations and duties. It also concerns their personal moral integrity, because a subjective interpretation of what is best for the patient also informs the ethical dimensions of practice as it concerns what the nurse believes ought to be done for patients in a normative sense. Diverse interpretations are also anticipated as the healthcare team, family, and patient may all have recommendations on what is best regarding a particular plan of care. It is vital to weigh and balance all of these considerations in order to mitigate tension between competing views, but arrive at a plan of care that supports advocating for the patient's best interests and quality care. Part of a unit's responsibility is advocating for a healthcare unit's ethical climate where concern about the ethical dimensions of practice may be acknowledged, explored, and potentially reconciled through discussion and appealing to further collaboration with other resources such as ethics committees and consultations. According to Olson (1998), the Hospital Ethical Climate Survey (HECS) was created to empirically quantify nurses' perceptions of their work environment's ethical climate and how an understanding of this climate influences their clinical practice and is informed by their perceived interactions with peers, patients, managers, the hospital itself, and physicians. The hope is that understanding an organization's ethical climate and how workers perceive it will ultimately aid nurses and managers in configuring ways to acknowledge and improve insufficiencies (Olson, 1998).

According to Pauly, Varcoe, Storch and Newton (2009), nurses in acute care hospitals in British Columbia had mean MDS scores that were negatively moderately 
associated $(r=-0.42, p<0.01)$ with mean Hospital Ethical Climate Survey (HECS) scores. A more positive score was indicative of a positive ethical climate, thus higher HECS scores indicated lower levels of moral distress. Furthermore, Sauerland, Marotta, Peinemann, Berndt, and Robichaux (2014) found in their mixed-methods study that a negative moderate association existed between the hospital ethical climate and moral distress $(r=-0.51, p<0.001)$. Additionally, Sauerland et al. (2014) identified three qualitative themes: caring for patients, the environment of care, and providers of care. Evidence from these studies reveals that individual levels of moral distress are influenced by a unit's ethical culture, and if it is negative, then it may be hypothesized that moral distress will increase.

Individual experiences of moral distress are influenced by organizational factors, such as a unit's culture, and their policy and procedures in addressing and acknowledging the ethical dimensions of practice. This has broader implications for units and organizations as they seek to retain staff and improve the quality of care of their patients. By addressing the ethical dimensions of practice in a transparent and collaborative way that is not prohibitive, but encourages transparent discussion, an ethically healthy organization with less moral distress is retained, because ethical issues are addressed and acknowledged directly.

According to Dudzinski (2016), moral distress may create a need for an ethics consultation, but the primary goal is not to decrease nurses' experience of moral distress. As noted earlier, coping with moral distress does not fix the cause or underlying moral issues occurring in practice (e.g., futile treatment at the end-of-life, staffing inadequacies, sub-optimal pain relief). While the distress a nurse feels is important and needs to be 
acknowledged, the underlying moral issue that is impacting the quality of patient care is a priority. Before alleviating individual-level experiences of moral distress, the goal is to identify and address the moral issues that cause moral distress in order to promote care that is in the patient's best interests. Dudzinski (2016) provides an example when a young patient who is capable of making decisions refuses life-sustaining treatment even though there is evidence that it will be effective. The nurse taking care of this particular patient may experience moral distress even though the plan of care remains ethical, because it is within the patient's right to choose their course of treatment. This unpacks some important aspects within the concept of moral distress. For example, if the nurse performs an inaccurate moral evaluation, such as believing that stopping treatment is wrong in this example, then it infringes on the patient's right to autonomy. Specifically, the patient's choice to discontinue treatment is not wrong, because it is in accordance with patient values and what the patient wants for their life. However, even though the patient is operating well within their rights, the nurse remains morally distressed and this situation may benefit from undergoing some ethical scrutiny to ensure that all parties are operating within their full mental capabilities and understand all assumptions. Also, it would be prudent to let the patient make such a decision after the patient understood the risks and had their questions and concerns addressed, because the provision of fully informed consent is essential.

Moral distress may linger with respect to an ethical dilemma, which is a situation where more than one course of action is ethically justified (Dudzinski, 2016). For example, moral distress differs from a moral dilemma. Specifically, a moral dilemma occurs when a healthcare professional is conflicted about the correct ethical choice in a 
particular clinical situation (Hamric \& Blackhall, 2007). In moral distress, the healthcare professional is certain about the correct ethical choice, but is constrained from taking that action (Hamric \& Blackhall, 2007). An example of one type of moral dilemma occurs when conflict arises between the commitment to promote the patient's best interests and the commitment to respect the patient's autonomy when the patient's wishes conflict with what the nurse believes to be in the patient's best interests (Webster \& Baylis, 2000). However, the potential for an ethical dilemma occurs when what the nurse believes to be right and in the patient's best interests actually diverges with what the patient wants. It is worth exploring all instances to ensure that moral issues are discovered, assessed, and evaluated. A moral dilemma occurs when there are obligations to pursue two or more conflicting courses of action and there is not an obvious reason to prefer one course of action over the other(s) (Webster \& Baylis, 2000). If a nurse knows the right action to take, but is unable to act, then this results in moral distress, which is not a moral dilemma where two choices are potentially viable. A nurse's moral evaluation of any moral issue should be challenged in order to ensure that it holds up to ethical scrutiny, but the discourse this creates is a far better context of addressing ethical problems than merely coping with one's distress individually and ignoring how quality of patient care is impacted.

According to Webster and Baylis (2000), this highlights the need for healthcare organizations to encourage ethics consultations and nurses to bring ethical concerns to the forefront of discussion in order to deal with them in a thorough way that encourages shared understanding and mutual respect. When the patient was exercising their rights to discontinue treatment in the earlier example, the nurse was still morally distressed. Thus, 
the experience of moral distress will never be truly eliminated, even in cases where the nurse may be misguided in their moral evaluation, but it may be managed in a way that deals with the moral issue by bringing it to the forefront of clinical discourse and also respects preserving the personal and professional moral integrity of the nurse.

Furthermore, it is hoped that the integrity of all other healthcare professionals, the family of the patient, and the nurse(s) advocate for the quality of patient care.

\section{Perceptions of Quality Patient Care and Moral Distress}

Healthcare systems may perpetuate structures that challenge nurses' professional obligations and personal moral integrity. According to Hamric and Blackhall (2007), nurses with high moral distress scores reported a more negative ethical climate in their ICU, lower satisfaction with quality of care, and less collaboration with physicians than nurses with low moral distress. Interestingly, high levels of moral distress demonstrated lower satisfaction with quality of care, thus, it is inferred that moral distress impacts perceptions of quality of care. Perhaps this is a result of nurses feeling that they are unable to act in some way to benefit the patient's quality of care.

A convenience sample of 461 participants who responded to the American Association of Critical-Care Nurses (AACN) e-newsletter and social media survey found that nurses who perceived positive quality of care demonstrated decreased moral distress (Hiler, 2018). In addition, Hiler (2018) found from a hierarchical multiple regression analysis that significant predictors of the frequency of moral distress included the practice environment, quality of care, and nurse characteristics. Importantly, this study revealed that moral distress is associated with perceptions of a healthy practice environment and care quality. Higher levels of moral distress frequency were reported when nurses 
reported that care was futile. Moral distress occurred most frequently when family members wished for prolonged care, but the nurse felt that it was not in the patient's best interests (Hiler, 2018). A limitation of this study is that the researchers only looked at moral distress frequency and not intensity. As discussed earlier, nurses are unable to leave the bedside in the ICU for a prolonged period of time during their workday, so it is important to not only explore the frequency of morally distressing events, but also their intensity and how this impacts their perceptions, experiences, and individual coping style. Thus, it is important to investigate the link between moral distress and nurses' perceptions of quality patient care as previous research is tenuous, because few studies have examined this connection explicitly. Importantly, the focus is on perceptions of quality patient care, not the actual quality of care itself. However, an investigation of how nurses cope with moral distress and moral residue and how the intensity and frequency of those experiences impacts quality patient care has yet to be conducted.

A combination of the frequency and intensity of morally distressing events occurring in the ICU may contribute to the crescendo effect of moral residue and impact nurses' perceptions of quality of care. As demonstrated by Hiler (2018), the frequency of moral distress relates to perceptions of quality of care. However, this is not consistent across all studies regarding what tends to be the driver of negative experiences. As stated earlier, disparate perspectives remain in the literature regarding the overall impact of the frequency and intensity of morally distressing experiences on individual nurses. As a result, it is important to explore nurses' coping abilities, because it may influence not only nurses' perceptions and experiences of the frequency and/or intensity of morally 
distressing events, but also how they cope with those events and then subsequently perceive the quality of their patient care.

DeKeyser and Berkovitz (2012) found from their study of 119 surgical nurses that lower levels of general quality of nursing care, nursing skill, and being able to meet the patient's needs were associated with higher frequencies of ethical dilemmas and moral distress. However, the intensity of the nurses' feelings was not related to quality of care. Hamric and Blackhall (2007) found that total moral distress scores were driven primarily by nurses' perceptions of the frequency of morally distressing situations. However, a limitation of DeKeyser and Berkovitz's (2012) study is that numerous other variables, such as personality variables, may impact moral distress and were not investigated. This is a limitation that this study will address by exploring nurses' coping and how it interacts with their perceptions of quality care, moral distress, and moral residue. How a person copes with problems and emotions is an insight into their personality. Personality characteristics and how constructive or maladaptive coping methods are employed in a personalized coping strategy is vital in understanding how the frequency and or intensity of a morally distressing experience impacts an individual.

According to Rochefort and Clarke (2010), 339 neonatal ICU nurses were recruited and working across 9 NICUs for this particular study. The sample reported on the Revised Nursing Work Index (NWI-R) (i.e., three work environment subscales) a statistically significant increase in nurse-appraised quality of care. Nurses' perceptions when transitioning from the worst to the best work environments noted an improvement in the quality of nursing care. Overall, findings from this study determined that work environment characteristics such as nurse staffing and resource adequacy, nurse- 
physician relationships, and nurse-management relationships are related to better outcomes for nurses and higher nurse-rated quality of care in NICUs. This demonstrates that a nurse's subjective assessment or perception of the quality of care provided is associated with an objective measure lending credibility to the accuracy of a nurse's perceptions of their experiences. The NWI-R is considered an objective measure, because the internal consistency of its subscales has been demonstrated repeatedly in prior research (Rochefort \& Clarke, 2010). This study was important in order to explore how nurse's perceptions and experiences not only impacted quality patient care, but also how moral distress, coping, and moral residue played a role.

According to McAndrew, Leske, and Garcia (2011), 78 critical care nurses from medical, surgical/trauma, neuroscience, and cardiovascular ICUs participated in a descriptive, correlational, prospective study to determine the level of moral distress in the ICU setting, the perception of the practice environment in the ICU, the relationship of moral distress to the practice environment, and what effect moral distress has on the delivery of nursing care. The MDS measured the intensity and frequency of morally distressing experiences and the Practice Environment Scale of the Nursing Work Index measured five areas: leadership and support, participation in hospital affairs, collegial relationships, nurse manager resource and staffing adequacy, and quality of care. Importantly, McAndrew et al. (2011) revealed that moral distress dilemmas that occurred most frequently were not situations that caused the greatest level of distress for nurses. For example, the most intensely morally distressing item was "Assisting a physician who in your opinion is providing incompetent care." Alternatively, the most frequently morally distressing item was "Carrying out a physician order for unnecessary tests and 
treatments." Importantly, the overall results of the responses on the older MDS revealed that the intensity of a morally distressing experience was reported more often than the frequency. Thus, the total score for intensity was higher than frequency. A composite score was not calculated.

Responses from nurses revealed that the frequency of moral distress impacts all aspects of professional practice except foundations for quality of care (McAndrew et al., 2011). Thus, moral distress was found not to affect the quality of nursing care provided. In contrast to other studies, the results did not support previous findings that moral distress does impact the quality of nursing care. Moreover, McAndrew et al. (2011) noted that the intensity of morally distressing experiences may be a result of the reduced collegial relationships in the ICU. The authors noted that future qualitative research was needed to understand how nurses' experiences and perceptions of the practice environment relate to the experience of moral distress and how that experience of moral distress influences the quality of care provided to patients and their families. Thus, based on these conflicting findings further research is warranted. This study addressed the limitations of this quantitative study: a limited focus on the context of morally distressing experiences. McAndrew et al. (2011) identified limitations that quantitative tools may not reveal the range of morally distressing experiences. Furthermore, implications of morally distressing experiences were highlighted; however, no information was available on the degree, significance, or pervasiveness of any of the experiences (McAndrew et al., 2011). Thus, this study sought to provide context that previous quantitative studies have not addressed with a focus on the quality of patient care. This study explored the perceptions 
and experiences of moral distress, moral residue, stress and coping, and perceptions of quality patient care in adult SICU nurses.

\section{Futile Treatment in the ICU}

Much of the research addressing futile treatment concerns issues of cost and limited healthcare resources. A guiding rationale that Fleck employed was considering quality of life judgments in a context of controlling cost and promoting effectiveness. It is arguable that while a 97-year old may survive a total implantable artificial heart they will not have the same quality of life as a 45-year old. Using Fleck's (2009) logic — funding the intervention for the 45 -year old is more effective because more life years are at stake. Granted this is an assumption that is discriminatory based on one's age. Quality of life here is somewhat subjective, because for some this may mean being able to see, talk, and hear, while for others it may mean being able to get out of bed in the morning and care for their basic activities of daily living - an example of different conceptions of the good life, which obviously varies from person-to-person and should not be imposed on anyone. Thus, this leads to an important premise: the "who" and "how" is biased and subjective regarding determinations for cost, effectiveness of therapies, and ultimately quality of life. As a result, it is impossible to account for the ethical dimensions of one patient over another without severely delimiting the care one patient gets due to competing limited healthcare resources. Fleck (2009) considered the subjective nature of his argument by claiming that not having the ability to access any portion of the normal opportunity range of our society is reason enough to justly deny individual's non-beneficial, aggressive treatments that are expensive. However, the individual denied therapy under the premise of it being costly and ineffective is robbed from a certain level of quality of life. 
However, issues of cost and limited resources also apply to the provision of futile treatment in the ICU. In particular, treatment that fails to provide some benefit or improve the person as a whole and only preserves a permanent state of unconsciousness or cannot end dependence on intensive healthcare was defined as futile treatment (Schneiderman, Jecker, \& Jonsen, 1990). Advances in technology have allowed futile treatment to persist because healthcare in the last 50 years has increased length of life but not necessarily quality of life — creating a disparity between healthcare resources and a life of functional vitality (Waldrop \& Meeker, 2012). Healthcare resources in a critical care context are limited, thus futile treatment of a patient at or near the end-of-life may impact the care of other patients in the ICU (Huynh, Kleerup, Raj, \& Wenger, 2014; Niederman \& Berger, 2010).

Situations of futile treatment may present a phenomenon known as opportunity cost. Opportunity cost is defined as "the loss of potential gain from other alternatives when one alternative is chosen, if critical care is unavailable for another patient for whom it is indicated" (Huynh et al., 2014, p. 1978). Healthcare reform aims to create an equitable and just distribution of medical care across the United States; however, opportunity cost is a reason why futile treatment should be minimized because it creates a strain on healthcare delivery through exorbitant costs and an inequitable distribution of resources (Huyn et al., 2014).

Futile treatment in the ICU is a common occurrence that ends up being costly. According to Niederman and Berger (2010), almost half of all patients that die in United States hospitals receive ICU treatment and some of this may have been futile. In a perfect world of unlimited resources, futile treatment decisions may be based on individual 
patient wishes without any consideration for other patients (Niederman \& Berger, 2010). However, in today's healthcare climate of pay-for-performance, the healthcare decisions implemented in one patient's care may affect other patients indirectly, thus futile treatment places a strain on healthcare delivery and cost (Niederman \& Berger, 2010). For example, the choice to give antibiotics to patients receiving futile care ultimately leads to antibiotic resistance that may make the use of antibiotics in other patients ineffective (Niederman \& Berger, 2010).

According to Huynh et al. (2013), critical care specialists in five ICUs were surveyed at an academic medical center in order to quantify the prevalence and cost of perceived futile treatment in the ICU. Results revealed that 123 patients were perceived as receiving futile treatment and 84 of those patients died before discharge, 20 died within six months of ICU treatment, and the remainder were in poor health (Huynh et al., 2013). Overall, the cost of futile treatment in the ICU was estimated to be at $\$ 2.6$ million (Huynh et al., 2013). Critical care perceived to be futile is all too common and associated with substantial costs (Huynh et al., 2013). For example, patients who suffer from chronic illnesses account for about $32 \%$ of total Medicare spending during the last two years of life (The Dartmouth Atlas of Healthcare, 2018). Futile care at the end-of-life not only puts a strain on the availability of healthcare resources for other patients, but also on the costs associated with treatment.

According to Reddy, O'Horo, Hoskote, and Moua (2015), any consideration regarding the specifics of futile treatment must be context dependent. For example, patient and family input are important in determining the goals and plan of care (Reddy et al., 2015). Patient and family goals should be acknowledged, communicated, and 
respected throughout the plan of care in order to avoid situations of futile treatment. Specifically, contextual understanding demands the following considerations: the conservation of limited healthcare resources, the consultation and implementation of palliative care at an appropriate time, and the provision of timely referrals to hospice as indicated by the patient and/or family (Reddy et al., 2015). Thus, patient care in the ICU demands that the healthcare team work together in order to communicate the best possible course of action that considers all perspectives in a timely manner.

However, consider if the family is adamant about continuing care even if the patient's prognosis is poor. Legally, the healthcare team has to appeal to the directives of the power of attorney in cases where the patient is incapacitated. Alternatively, if that care is immoral, because it promotes suffering, pain, and lingering without any chances of a return to a life of functional vitality off of a ventilator, then an ethical dilemma presents itself. It is evident that nurses in these types of situations may experience moral distress for their complicity in actions dictated by families, which does not help in considering the immorality of actions that are deemed legal by our justice system. This stresses the importance of healthy work environments that promote discussion about the ethical dimensions of practice.

A study of nurses who worked in nursing homes and acute geriatric units revealed that situations of futile and inadequate treatment at the end-of-life were associated with moral distress (Piers et al., 2012). In particular, nurses reported that most moral distress was experienced in situations where unjustifiable life support measures were used, unnecessary tests and treatments were conducted, and incompetent staff were employed (Piers et al., 2012). Moral distress in critical care nurses related to futile treatment has 
been associated with age (i.e., greater than 33 years of age), time in the unit (i.e., greater than four years) and nursing experience (i.e., greater than four years) (Mobley, Rady, Verheijde, Patel, \& Larson, 2007). These results may demonstrate the inadequacy of coping mechanisms among critical care nurses in morally distressing situations because of repeated involvement in futile treatment during the course of their nursing careers (Mobley et al., 2007).

The clarity of life and death is obfuscated by the ICU environment's capacity to sustain life almost indefinitely through advancements in healthcare technology. If patients stop breathing, then they are intubated with an endotracheal tube and supported with a ventilator. If patients' kidneys shut down, they are dialyzed with tubes, filters, and machines. If patients contract infections from hospital- and/or community-acquired bugs, they are treated with a salvo of antibiotics that further impairs their immunities and may lead to antibiotic resistance in the process. The ICU is a place where the existence of brain death requires multiple tests and multiple physicians' to clinically diagnose a patient as dead. Often the treatments carried out on patients prior to their death contributes to their suffering and nurses are unable to describe their individual pain, suffering, and functional quality of life, because they are in a hospital bed full of drugs, drains, tubes, and attached to monitoring equipment that fails to adequately paint the picture of who the person used to be and wanted to be. Moral distress is common to the ICU by nature of the care provided in that type of environment where it is often difficult to predict an outcome with any degree of certainty. Suffering creates a death that is not respectful when it denies focusing on considerations such as quality of life, the 
consequences of the purported "effective" therapy, and the family's expectations about the goals of care and what it means for the future of their loved one.

\section{Moral Distress at the End-of-Life}

By virtue of caring for patients at the end-of-life, nurses should be consistently involved in the critical decision-making process between other healthcare team members and the family of dying patients. In contrast, according to Flannery, Ramjan, and Peters, (2016), nurses tend to have an informal role in end-of-life decision-making. In particular, there is lack of a standardized approach to end-of-life decision-making, and inclusion of the nurse is often dependent on the physician's decision to include or exclude their involvement with the family of ICU patients (Flannery et al., 2016). Due to lack of a clearly defined role in the decision-making process at the end-of-life in the ICU, nurses may experience moral distress, because of their lack of involvement in critical decisionmaking, even though they have had more patient and family contact than any other member of the healthcare team (Flannery et al., 2016; Hamric et al., 2012; Robinson, 2010). As decisions are made to continue treatment or consent for procedures that will only add a marginally beneficial outcome, nurses are a part of those treatments and witnesses to those consents, there is no opportunity for nurses to provide input and recommendations to advocate for patients' best interests and quality of life.

Providing end-of-life care to patients at or near the end-of-life may be the most morally distressing event for patients, families, and healthcare providers, because of the potential for disagreements regarding the ethical nature of care (Taylor-Ford, 2013). Low levels of moral distress were reported from a convenience sample of 105 nurses from a medical-surgical intensive care unit and a transitional care unit (Wilson et al., 2013). 
However, the highest levels of moral distress were reported in situations related to futile treatment at the end-of-life (Wilson et al., 2013). According to St Ledger et al. (2013), the main goal of ICU treatment "is to help patients survive acute threats to their lives" (p. 1870). However, this is not always appropriate in situations where treatment is considered futile, so difficult decisions about de-escalating care or withdrawing active treatment need to be made (St Ledger et al., 2013). Particularly, the ICU may perpetuate a moral gray zone as a result of advanced healthcare technologies and treatments that postpone life without accounting for any quality of life.

Knowing the right course of action is different from being able to consistently carry it out in the clinical healthcare setting. A survey of 277 critical care nurses responded to the MDS and results revealed that moral distress frequency was moderately and negatively associated with nurses' collaboration in end-of-life patient care conferences (Browning, 2013). Importantly, the MDS is an older version of moral distress instruments. The MDS-R is the updated version of moral distress quantitative measurement and may be used in other disciplines and in multiple settings, not just the ICU. Thus, studies that use the MDS are limited in that they are not using the most updated measurement for moral distress. This association suggests that the more nurses collaborate in end-of-life patient conferences, the lower their frequency of moral distress experiences. Interestingly, according to Whitehead et al. (2015), 592 clinicians completed the MDS-R and providers who had received end-of-life care training, 85.4 (51.9); MDS$\mathrm{R}$ mean (standard deviation); had higher average levels of moral distress than those without the training, $72.0(48.1), p=.005$. This finding may illuminate the increased discord between providers who learn evidence-based practices in end-of-life care but are 
unable to implement them in certain clinical settings (Whitehead et al., 2015). This is in accordance with the basic tenant of moral distress, which is a "perceived inability to act on one's moral obligations and values" (Whitehead et al., 2015, p. 123). Thus, as providers increase their knowledge about evidence-based practice, their moral distress may increase because they cannot act in accordance with their new understanding in a practice setting due to institutional constraints, physician's orders, or family's wishes (Whitehead et al., 2015). This exemplifies the dissonance inherent in the healthcare system, because the more nurses learn about how they ought to treat patients via evidence-based practice, the more nurses may learn how severely limited they are in enacting those practice guidelines due to the culture and environment of the ICU.

Futile treatment provided to a patient on an end-of-life trajectory may impact nurses more negatively than other ethical considerations at the end-of-life. As stated earlier, Piers et al. (2012) found that the highest level of moral distress among nurses caring for geriatric patients near the end-of-life was caused by unjustifiable life support, unnecessary tests and treatments, and working with incompetent colleagues.

Alternatively, requests for euthanasia and increased morphine doses in an unconscious patient that were believed to hasten death were least likely to cause moral distress among these nurses (Piers et al., 2012). The provision of futile treatment contributes to moral distress more than euthanasia and perceptions of hastened death in unconscious patients in geriatric end-of-life care (Piers et al., 2012). This finding also suggests that symptom management is more important to nurses despite its perceptions of being ethically wrong such as providing pain medicine to hasten death in an unconscious patient near the endof-life. 


\section{Moral Distress and Conscientious Objection}

In order to reinforce the negative phenomenon of moral distress it is important to note that the experience of moral distress itself repeatedly serves as an alarm bell by indicating that morally problematic courses of action are being pursued (e.g., aggressive treatment at the end-of-life) in the clinical setting (Weber, 2016). Interestingly, moral distress creates insight into the gray zones that perpetuate evil intentions in caregiving situations in the healthcare environment (Davion, 2006). These critical events that lead to moral distress are gray instead of "black and white" because the nature of their occurrence — what should be done in particular instances, and how they should be addressed—is rarely a simple solution. For example, nurses are not able to make just and moral decisions in caring for patients at the end-of-life, because the treatments they are complicit in providing prolong dying and carry the weight of various considered moral judgments. According to Davion (2006), a system that promotes the culpable infliction of intolerable harm is considered evil. This infliction is imbedded in the nature of the ICU clinical environment when staff are forced to provide medically futile treatment, operate with staffing and patient ratios that are inadequate and unsafe, and work in a healthcare team where communication is fractured, deferred, and inconsistent. These end-of-life treatment decisions create situations in which nurses are complicit.

The ICU clinical environment creates some conditions that affect decisionmaking at the end-of-life. For example, treatment decisions that place nurses at risk for moral distress and the subsequent consequences of this negative experience may include burnout, turnover, and conscientious objection and may be a result of poor healthcare team communication, short staffing, resource shortages, poor ethical climate and poor 
collaboration (Epstein \& Hamric, 2009; Hamric, 2014). Additionally, moral distress is a serious indicator of morally wrong decisions that promote unethical treatment of patients at the end-of-life by prolonging the dying process (Epstein \& Hamric, 2009; Hamric, 2014).

In this case, conscientious objection may be viewed in two ways: (a) situations that continually promote a response of conscientious objection are harmful, because the critical events that occur in the ICU leading to this decision are indicative of an ethical climate where immorality is pervasive and moral wrongdoing is commonplace; (b) the option to conscientiously object is a relatively little explored concept and is warranted in order to promote the well-being of the patient and prevent complicity of the nurse (Epstein \& Hamric, 2009; Hamric, 2014; Weber, 2016). A consequence of conscientious objection is the patient who is ignored by a provider's justification to avoid treatment. In this particular case, the patient's autonomy is challenged by the nurse's refusal to provide care perceived to be immoral. However, in today's healthcare system with advances in technology, the prevalence and increased incidences of moral distress describe a clinical environment where moral issues are often commonplace. It is vital for nurses to have conscientious objection as an option, because it demonstrates the importance of ethical decision-making at the bedside.

According to Davis, Schrader, and Belcheir (2012), from a study of 1144 nurses across Idaho, nurses whose ethical beliefs were informed by religious beliefs had higher levels of moral distress and greater differences in instances of conscientious objection than nurses whose beliefs were informed by family values, life and work experience, political views, or professional code of ethics. Furthermore, $76 \%$ of the respondents felt 
that patients' rights to healthcare were more important than nurses' rights to conscientious objection, but this view was uncertain if they were working alone (Davis et al., 2012). For example, $66 \%$ of the nurses felt that if they worked alone in a small or rural setting, they should not be responsible for providing care to patients if it went against their religious or moral values when the situation wasn't a life-threatening emergency (Davis et al., 2012). Interestingly, 35\% of the respondents revealed that they experienced moral distress in the workplace at least once a month. This poses important implications for nursing practice in that there is a conflict between the rights of nurses and patients. Inevitably, nurses that deny treating patients on religious grounds also deny the patient's autonomous rights, which is immoral. This is just one particular caveat in a complex, diverse argument that challenges the autonomous rights of patients. According to Berlinger (2016), "So this is moral distress, caused by a situation that troubles the conscience but is not resolvable, conceptually or practically, the mechanism of opting out” (p. 33). As part of their professional standards of practice, ICU nurses must advocate for the patient's best interests and quality of care. However, as part of their personal moral integrity they may feel powerless, because they are continuing to contribute to the patient's suffering and harm by providing treatments that sustain life.

Nurses are bound by their professional code of ethics to advocate for the safety and well-being of their patients. According to the ANA (2015) Code of Ethics for Nurses with Interpretive Statements: "Nurses are responsible for contributing to a moral environment that demands respectful interactions among colleagues, mutual peer support, and open identification of difficult issues, which includes ongoing professional development of staff in ethical problem solving" (p. 24). End-of-life care in the ICU may 
be morally problematic, because it may threaten the well-being of patients through futile treatment. Nurses are able to act as alarm bells for unethical treatment of patients through their experience of moral distress that consequently may manifest as conscientious objection. Conscientious objection enables the nurse to mitigate feeling complicit in futile treatment by calling attention to immoral clinical decision-making and treatments that occur by nature of end-of-life care in the ICU. Importantly, healthcare systems and ICUs must create avenues for staff to acknowledge when immoralities are occurring in order to prevent harm. Specifically, nurses should be allowed to conscientiously object to care that may cause harm to the patient. However, conscientious objection becomes a problem when the rights of the patient are harmed by the actions of a nurse refusing to provide care. This is an ethical dilemma that would require an ethics consultation in order to prevent undue harm to the patient.

It may not be acceptable for the nurse to request from the charge nurse a change of assignments when feeling morally distress when the patient is still receiving medically futile treatment. For example, if staffing is short, then it makes changing assignments difficult or not possible. The question becomes how best may a nurse who is morally distressed not only acknowledge their own moral distress regarding an immoral wrong at the bedside, but put a plan of action in place to persistently address this ethical dimension of practice within the interdisciplinary healthcare team, while also still providing care in the patient's best interests.

The need to use conscientious objection is a particularly salient point that intersects the moral resiliency debate within the moral distress literature. According to Carse (1991), a good healthcare professional should be able to summon the appropriate 
degree of emotional detachment, or level-headedness, when this is vital to providing service to the well-being of the patient. For example, one way that someone could disagree with the need for conscientious objection is the idea of moral resiliency, because providers ought to be adaptable and flexible to the moral challenges of their work. According to Lachman (2016), it is this ability to adapt to changing circumstances with a good sense of humor that is crucial to flexibility. However, self-confidence and moral courage are not enough to prevent immoral wrongs from occurring at the bedside. The care and well-being of the patient and family are of utmost importance. Importantly, healthcare professionals should not tolerate evil or immoral wrongs that occur at the bedside, such as medically futile treatment, inadequate staffing ratios that lead to unsafe care, and poor team communication by being morally resilient. Alternatively, ignoring immoral problems by attempting to be impartial and compartmentalize values, roles, and obligations does not facilitate the nurse's prevention of "taking their work home", or refusal to conscientiously object to treatments deemed harmful to the patient's quality of care.

Conscientious objection should be justified and defended by supporting one of two possible options (Kantymir \& McLeod, 2014): (1) the objection is reasonable, "in particular by showing that what grounds the objection is as likely or more likely to be true than what grounds the standard of care for patients" (p. 21); and (2) the objection is genuine, in addition to satisfying the following criteria: "patients will still get the care they need in a respectful and timely fashion, any empirical beliefs on which the objection rests are not baseless, and the moral or religious beliefs on which it rests are not discriminatory" (p. 21). But this objection is unconvincing, because it is unclear how a 
nurse's moral distress and threatening of their personal moral integrity may be an empirical belief. Furthermore, moral or religious beliefs should not be discriminatory, but moral distress from threatened moral integrity in a clinical situation relates to a potential harm being done to the patient that the nurse has recognized through her moral distress. Thus, the patient may still get care they need; however, the nurse, by choosing to conscientiously object, is choosing not to harm the patient, because they fear that what is being ordered is unethical and not right. In particular, Kantymir and McLeod's (2014) conscientious objection only applies to management, while ignoring the labor injustice experienced by the individual nurse at the bedside who is responsible for carrying out the physician's orders in the context of a hierarchical power differential.

The nurse's experience of moral distress during futile, end-of-life treatment in the ICU is an appropriate rationale to warrant their conscientious objection and thus, warrants investigation into the immorality of ineffective and costly treatments that contribute to the prolonged dying and suffering among ICU patients at or near the end-oflife. Nurses should have to justify their conscientious objection due to their experience of moral distress and refusal to continue being complicit in their perceptions of patient harm. Thus, they have an obligation to justify and explain refusals of conscience. Nurses' experiences of moral distress ought to signal the team/unit and organization to investigate these instances, because it is worthy enough to warrant an investigation into the ethical dimensions of practice. This does not absolve nurses of being able to participate in an ethical discussion during an interdisciplinary healthcare team meeting in order to state the impetus for moral distress. However, the pressure of having to justify one's reasons should not be prohibitive or discriminatory. Through safe and morally reflective safe 
spaces, the healthcare team, family, and patient may use education, communication, and collaboration regarding the ethical dimensions of practice to arrive at a solution that may not be agreeable for everyone (Hamric, 2014; Hamric \& Wocial, 2016) but at least a rationale for a course of action is sought and arrived at collectively. In this way, parties would be allowed to arrive at a consensus by publicly acknowledging and addressing their questions, concerns, and reservations.

\section{Moral Distress and Burnout}

A close association between the experiences of moral distress and burnout among ICU nurses has been demonstrated in the literature. According to Cherniss (1980), burnout is conceptually defined as "a process in which the professional's attitudes and behavior change in negative ways in response to job strain" (as cited in Leiter \& Harvie, 1998, p. 1611). Nurses' elevated levels of moral distress were related to measures of burnout such as emotional exhaustion (Piers et al., 2012). In a cross-sectional survey of 114 nurses from neonatal/pediatric, oncology, and critical care units, researchers found that moral distress was a statistically significant predictor of the three aspects of burnout including emotional exhaustion, depersonalization, and personal accomplishment using the Maslach Burnout Inventory (MBI) (Rushton, Batcheller, Schroeder, \& Donohue, 2015). Emotional exhaustion is a feeling of being overextended and exhausted by the nature of one's work (Vahey, Aiken, Sloane, Clarke, \& Vargas, 2004). Depersonalization is characterized by an impersonal response toward recipients of the nurses' care (Vahey et al., 2004). Finally, reduced personal accomplishments are the feelings of incompetence and unsuccessful achievement that nurses feel regarding their work with patients (Vahey et al., 2004). 
The conceptual definition of burnout matches its operational definition. Burnout is a job-related syndrome characterized by the operational definitions of emotional exhaustion, depersonalization, and feelings of reduced personal accomplishment (Vahey et al., 2004). Conceptually, burnout is composed of exhaustion, cynicism, and a lack of professional efficacy (Leiter \& Harvie, 1998), which is in accordance with the operationally defined burnout syndrome measured by the MBI (Rushton et al., 2015). Exhaustion is the reduction of one's physical and emotional resources, cynicism is described as indifference towards one's work, and lack of professional efficacy refers to the lack of competence and accomplishment with one's work (Leiter \& Harvie, 1998). From a survey of 605 patients and 711 nurses, findings indicated that patients on units where nurses felt that their work was meaningful were more satisfied with their hospital stay; however, patients from units where nurses were exhausted or frequently expressed their desire to quit were less satisfied with the care provided (Leiter \& Harvie, 1998).

Additionally, Johnson-Coyle et al. (2016) found from their prospective crosssectional survey of healthcare professionals working in the cardiovascular intensive care unit, that moral distress and burnout scores were positively associated $(p<0.001)$ and both were negatively associated with job satisfaction $(p<0.001)$. Furthermore, JohnsonCoyle et al. (2016) found that their data revealed that issues related to lower satisfaction were associated with how the ICU was being managed, to workplace recognition, and "being heard" in the workplace. These issues have important implications for reforming the way the ethical dimensions of practice are acknowledged at the unit/team level and system culture. If management provides a way for nurses to effectively voice their ethical 
concerns through policy, then they will more effectively recognize the individual nurse in their concern, which may mitigate moral distress and burnout.

Nurses' experience of burnout may also have implications for patient satisfaction. A cross-sectional survey of 820 nurses and 621 patients from 40 units in 20 hospitals across the United States was conducted and found that if patients were cared for on units that the nurses described as adequately staffed, supported administratively, and having friendly relations between physicians and nurses, then they were more than twice as likely as other patients to say they were satisfied with their care. These nurses noted statistically significantly lower burnout scores on the MBI and that overall levels of nursing burnout in hospital units affected patient satisfaction (Vahey et al., 2004). In addition, a recent review of the literature found that nurses' experience of moral distress is often associated with difficult care situations, and that these nurses were more likely to experience burnout, which might affect their professional roles (Oh \& Gastmans, 2015). Thus, if nurses are experiencing intense levels of moral distress, then burnout and eventual resignation or conscientious objection might be potential consequences as postulated by the crescendo effect. The transition from an experience of moral distress to a piling up and tipping point of unmanageability is not completely understood. Thus, this study was important, because it not only explored the perceptions and experiences of SICU nurses' moral distress, moral residue, and stress and coping, but also quality patient care.

\section{Identifying the Gaps in the Literature}

This study will explore how the perceptions and experiences of moral distress and moral residue among adult surgical ICU nurses are influenced by stress and coping, 
perceptions of quality care, and root causes of moral distress. This study will provide knowledge to fill identified gaps such as how the crescendo effect is impacted by root causes of moral distress, moral residue, coping, and perceptions of quality patient care. It is unclear if the crescendo effect is a linear sequence of events that develops from moral distress to moral residue, or if manifestation of the crescendo effect develops from frequency or some combination of the intensity of the moral issues. The context of moral distress and moral residue will be explored by asking questions to reveal the how, why, when, and where of these two concepts. This study adds to the increasing body of nursing knowledge by targeting the context of the phenomenon. Exploring how moral distress is impacted by context, such as time, intensity, and frequency of the experience is crucial for intervention and instrument development. The descriptive qualitative approach seeks to understand participants' firsthand accounts through a wide-ranging summary in their own words (Sandelowski, 2000).

A gap in the literature was identified regarding the absence of addressing moral distress among young nurses who begin a career in nursing. For example, studies were not found that followed nurses immediately out of nursing school who started in the ICU longitudinally in order to assess for the development of moral distress over time. Their moral distress should not be blamed on ineffective coping skills, because central to moral distress is a perceived moral wrong that occurred, because the nurse was unable to change the course of action. This phenomenon harms patients and the providers of care, thus their moral distress as an indicator of a moral issue and whether or not they cope effectively is tangential to the immorality occurring at the bedside. However, how nurses 
cope is of interest, because it may impact the quality of patient care delivered.

Importantly, moral distress is occurring, because moral issues remain unchallenged.

Several important gaps related to moral distress and moral residue were noted by Epstein and Hamric (2009), which this study sought to address. First, despite the available research, there are no studies on moral residue. Second, the crescendo effect was created from interviews with both physicians and nurses, but largely it has come from nursing data. Third, it is unclear whether or not the crescendo effect may be generalized to non-ICU settings. Fourth, gaps in the crescendo effect remain regarding how time or intensity plays a role. The number of years or contacts with moral distress required to cause a professional manifestation of moral residue and the crescendo effect is unclear. Additionally, it is also uncertain whether the intensity or frequency of particular root causes increase moral distress and if this is a linear pattern. Fifth, it is unknown how moral resilience, ideology, spirituality, compassion fatigue, knowledge of ethics, or other characteristics influence the crescendo effect. Sixth, there is no moral residue instrument to measure the phenomenon.

It is important to note that this study will not identify all of the gaps revealed by Epstein and Hamric (2009). This study addressed significant gaps in the literature by exploring root causes of moral distress, nurses' coping mechanisms, and how perceptions and experiences of moral distress and unresolved moral residue build into a crescendo effect. This study identified the first gap noted by Epstein and Hamric (2009) by exploring moral residue in adult ICU nurses across two SICUs. The fourth gap was addressed by asking nurses about the intensity, frequency, context, and timing of morally distressing root causes. The fifth gap was addressed by exploring nurses' coping 
mechanisms, particularly maladaptive and/or constructive ones, and how they work to reduce one's daily stress and impact perceptions of quality patient care. The sixth gap was addressed, because this study's findings may aid in the future development of a measure of moral residue. Understanding these perceptions and experiences in the adult ICU context across two SICUs was a novel approach to describing and interpreting moral distress, moral residue, and the crescendo effect.

More research about moral distress is needed in order to develop interventions aimed at reducing its negative impact among nurses (O’Connell, 2015). Such research will help nurse scientists define, describe, and understand the phenomenon as it applies to ICU nurses caring for patients in a variety of morally distressing scenarios. By better understanding how the concept of moral distress manifests in nurses' perceptions and experiences researchers will be able to identify, recognize, and potentially alleviate their experience of moral distress through interventions that allow bedside nurses to include ethical language in describing their experiences that are validating and able to remedy the structures that enable them through dialogue and persistence. Qualitative research will provide the foundation necessary for future research in moral distress interventions aimed at addressing the systemic nature of moral issues at the individual, unit, and system level.

\section{Summary}

This review of literature has focused on interrelated concepts, including: moral distress; healthcare rationing; quality of life, effectiveness of therapies, and cost; hospital organizational ethical climate and ethical dimensions of practice; perceptions of quality patient care and moral distress; futile treatment in the ICU; moral distress at the end-oflife; moral distress and conscientious objection; moral distress and burnout. In addition, 
gaps in the literature were identified. Given the current state of the science of moral distress and moral residue as it applies to the SICU clinical environment, it is important to explore the interrelationships among selected concepts in this investigation.

Particularly, because moral residue is poorly understood, a qualitative study that explores ICU nurses' perceptions and experiences of moral distress, moral residue, stress and coping, and perceptions of quality patient care is warranted.

Based on the theoretical assumptions of the crescendo effect and stress and coping theory, the interrelationships among concepts with moral distress and moral residue as it applies to SICU nurses were explored in the literature. For example, moral distress is a negative phenomenon that impacts a nurse's ability to provide the care they feel is "right". However, conceptual ambiguity of moral distress has resulted in a concept that is often viewed as a positive occurrence where moral resiliency may be harnessed to rectify the nurse's powerlessness. In doing so, the concept has been expanded, which has diluted its negative impact and softened the focus on the underpinning moral issues. Thus, this review of literature provides a renewed focus on moral distress and moral residue as symptoms of an underlying negative moral issue, which needs to be addressed in order to promote the primary goal of quality patient care that advocates for the patient's best interests. Thus, the main goal of research is not to relieve the nurse's moral distress or focus on the negative emotional reaction. Rather, the goal of moral distress research in the context of this study is to emphasize the experience of the individual SICU nurse. By listening to and observing experiences of moral distress and moral residue among nurses, a better understanding of the issues surrounding moral distress may be addressed and prevented in order to improve quality patient care. A potentially indirect effect is the 
improvement in nursing morale and satisfaction with their work, because their distress has been heard and acknowledged.

Healthcare rationing, quality of life determinations, effectiveness of therapies, and cost are all concepts that potentially impact a nurse's experience of moral distress. For example, a demonstrated root cause of moral distress in the literature, particularly relevant in the MDS-R instrument, is inadequate use of resources through inappropriate, aggressive care. The wasting of healthcare resources brings about more meta-healthcare concerns such as rationing of therapies, treatments, and medications. Rationing in this sense concerns making decisions about who gets what particular treatment based on a host of factors. These include the effectiveness of the purported therapy, cost, the probability of a successful and unsuccessful outcome, the wishes of the family in the event the patient is incapacitated, and consideration for others who would benefit from care.

Regarding this latter point, rationing should consider the patient under consideration and the counterfactual, or another patient who would be getting care that represents an infinite number of possibilities other than the specific patient receiving futile care. This is important because if one patient receiving futile care is considered to "rob" other healthier patients of potentially beneficial treatments, then this is considered a morally distressing experience because the nurse is powerless to mitigate cost constraints and advocate for treating patients with a better long-term prognosis. Importantly, issues of ageism and other discriminatory prejudices may come to light and ought to come under ethical scrutiny. 
Quality of life determinations are vital, particularly when therapies are deemed costly and have numerous potentially damaging side effects. The effectiveness of therapies is based on prior statistics of survival rates compared to the patient's case and a determination is made on whether a risky procedure may result in some reasonable return to a subjectively determined quality of life. Healthcare costs are a concern for nurses, patient care units, and healthcare systems, particularly as healthcare organizations operate with high commodity goods that are also limited resources. Thus, all of these administrative and meta-healthcare concepts influence moral distress and moral residue among nurses. Nurses are concerned about how resources are used in the healthcare setting, particularly as they witness the ineffectiveness of those therapies in cases of patients with a poor prognosis or where care is deemed aggressive and non-beneficial or futile.

Research has demonstrated that moral distress is associated with a hospital's organizational ethical climate, which concerns the ethical dimensions of practice and whether or not a hospital organizational culture advocates for addressing ethical issues or not. Furthermore, research has demonstrated that perceptions of quality patient care have been associated with moral distress. However, there are conflicting reports regarding experiences of moral distress and whether or not it actually impacts quality patient care. Thus, crafting quality of care concerns into this study on moral distress and moral residue was important, because it may have potentially impacted how nurses viewed and cared for their patients. Thus, if the hospital's organizational culture does not promote an active discussion of ethical issues, then it would be difficult to discuss moral distress for fear of 
prohibitive structures that do not encourage active reporting of moral issues as they occur in practice.

One of the ways to identify moral issues as important and meaningful is to acknowledge and investigate individual experiences of nurses' moral distress and moral residue. Furthermore, futile treatment in the ICU has been linked to experiences of moral distress as a prevalent experience at the end-of-life; thus, it was anticipated that futility is an appropriate concept to guide exploration of moral distress in adult SICU nurses. SICU nurses in particular were found to have some of the most maladaptive coping mechanisms, and this may be due to the nature of their patient population and the type of care they provide, which necessitates further exploration. Not all cases of moral distress deal with futile care or end-of-life circumstances, but it is worth noting that these circumstances are included in many occurrences of moral distress for nurses, and necessitates a qualitative exploration of the concept. Importantly, determining how the frequency, intensity, timing, and context of morally distressing situations impact the experience of SICU nurses is worth investigating, because it may illuminate how nurses manage the experiences through stress and coping and how the quality of patient care is impacted, as well as guide development of appropriate interventions.

A paucity of research has sought to explore the connection of moral distress and conscientious objection, particularly in the SICU environment. Thus, as a relatively unexplored concept in the adult SICU nursing literature investigation is warranted as a potential legal and ethical option for nurses and must be considered with respect to the patient outcomes and the quality of care. Importantly, the nuance of conscientious objection is that a nurse, who is responsible for the patient's best interests, denies the 
patient further care, because the task they are being ordered to do by a physician may potentially harm the patient. It is important to investigate the reason for conscientious objection in order to determine the underlying impetus for the nurse's decisions and whether it holds up under ethical scrutiny and is not merely discriminating against the patient, because they hold different beliefs or views from the nurse.

Interestingly, research has revealed that moral distress has been associated with burnout among nurses. Burnout as a consequence of moral distress is an important concept, because sustained moral distress resulting in feelings of powerlessness may lead to the nurse experiencing cynicism about his or her role. In particular, the professional role of the nurse may conflict with the goals of the healthcare system, because if hospitals cannot advocate for the patients they treat by listening to nurses and other frontline healthcare providers about moral and ethical patient care issues, then they will not be able to maintain ethically conscientious care that seeks to advocate for the patient's best interests and quality of care. Thus, with the conceptual considerations addressed in this review of literature, gaps in the literature were identified that lacked an exploration of how adult SICU nurses' perceptions and experiences of moral distress, moral residue, stress and coping, and quality of care impacted their lives on a daily basis. Thus, this study sought to address these conceptual concerns, particularly, because burnout and conscientious objection may be negative consequences of the phenomenon of moral distress and moral residue that damages nurses' moral agency.

Jonsen (1991) created an analogy between hot air balloons and bicycles where the balloons represented ethical theory and the bicycles represented practical every day ethical judgments. He made the important point that, "the weight of any ethical 
consideration comes, not from the principles or maxims invoked, but from the more factlike considerations that are piled onto practical judgment" (Jonsen, 1991, p. 15). Thus, appealing to everyday practical judgment informed by open communication and interdisciplinary healthcare team collaboration is a better fit for changing the ICU environment than abstract ethical theories that attempt to justify action through some prevailing universal moral principle. Care in the ICU clinical context has multiple variables to consider, but it ought to be responsive to the practical emotional and relational concerns of the family members and patients in order to prevent futile treatment of loved ones. Specifically, from the healthcare provider's perspective the conservation of limited healthcare resources, the consultation and implementation of palliative care at an appropriate time, and the provision of timely referrals to hospice as indicated by the patient and/or family (Reddy et al., 2015).

At the bedside, it would be difficult and unnecessary for the nurse to discuss the cost and limited nature of healthcare resources to a grieving family member of a loved one dying in the ICU - exhibiting the gross immorality of the healthcare system simply due to the implications of such a conversation. In this context, discussion of palliative care services and conversations regarding what the patient wanted at the end-of-life would be a more appropriate direction to steer the dialogue, because it values the relationships involved. Furthermore, that is why education, communication, and collaboration between the healthcare team, patient, and family are crucial not only to mitigating the nurse's moral distress, but also to addressing the culture of futile treatment in the ICU. Fostering these interventions by hospital administrators will potentially change the organizational culture, because it shifts the focus onto the ethical dimensions 
of practice and will improve quality of care for patients. This transcends futile treatment at the end-of-life and encompasses the myriad of root causes of moral distress that contribute to nurses feeling powerless and unable to change the direction of clinical practice into positive moral directions. Thus, the challenge for healthcare organizations and systems is to help create policies and procedures to alter this direction that healthcare has taken.

In conclusion, this study was innovative, because it used a descriptive qualitative approach to explore the following interrelated concepts: moral distress, moral residue, stress and coping, and perceptions of quality patient care from the perspective of SICU nurses in order to gain an understanding of the ethical dimensions of practice. Importantly, by considering the temporal, contextual, and situational influences of moral distress and moral residue, the intensity and frequency of moral distress among individual SICU nurses will be explored to determine the contribution to a professional and/or personal manifestation of moral residue. This study provided a focus on the context that quantitative studies fail to recognize. Findings from this study will help guide future intervention development related to identifying and acknowledging ICU nurses’ experiences of moral distress at end-of-life care and other root causes, such as shortstaffing, inadequate pain relief, and poor communication. These moral issues contribute to the nurse's perceptions and experiences of powerlessness. Implications for practice include creating a vision for change by developing organizational policy that will support staff in identifying and mitigating moral distress through potential nurse-led initiatives. Knowledge from this study will inform future research aimed at identifying and 
acknowledging the experience of moral distress and moral residue for adult SICU nurses and other healthcare providers. 


\section{CHAPTER 3 METHODS}

\section{Design}

A descriptive, qualitative approach explored the perceptions and experiences of moral distress, the root causes of moral distress, moral residue, coping, and perceptions of quality patient care among adult SICU nurses. This study was conducted through the lens of individual nurse participants using their own language and through their experiences in everyday events, which is required to clarify how moral distress manifests from root causes inherent in healthcare systems and units/teams and piles up to create moral residue and eventually lead to a crescendo effect (Epstein \& Hamric, 2009; Hamric, 2014; Sandelowski, 2000). This method was chosen due to conceptual ambiguity of moral distress. "Qualitative description is especially amenable to obtaining straight and largely unadorned answers to questions of special relevance to practitioners and policy makers" (Sandelowski, 2000, p. 337). The method explores concerns, responses, facilitators, and barriers, which reveals the who, what, and where of moral distress, and it is the "... method of choice when straight descriptions of phenomena are desired" (Sandelowski, 2000, p. 339) and the method is descriptive and interpretive. Moreover, "There is no mandate to produce anything other than a descriptive summary of an event, organized in a way that best contains the data collected and that will be most relevant to the audience for whom it was written" (Sandelowski, 2000, p. 339). This is salient for the conceptual clarification of moral distress, emphasizing a phenomenon that negatively 
effects providers due to challenges to moral integrity, and requisite focus on antecedents (e.g., clinical situation, internal and external constraints, moral issues) and consequences (e.g., withdrawing from the ethical dimensions of practices). However, the researcher bracketed assumptions and biases so that participants were not led toward focusing on the negativity of moral distress or any other particular aspect of the phenomenon. Instead, the adult SICU nurses' experiences were explored as openly as possible. Thus, a " $\ldots$ comprehensive summary of events in the everyday terms of those events" (Sandelowski, 2000, p. 334) was explored using this method. The investigator was tasked with making “... something of their data" (Sandelowski, 2010, p. 79). According to Glaser's (1978) classic work on the methodology of grounded theory, there is an important consideration that must be maintained: theoretical sensitivity. This study used a qualitative descriptive methodology, not grounded theory, in order to focus on nurse participants' factual descriptions of events (Sandelowski, 2000). According to Richards and Morse (2013), a mindset of theoretical sensitivity enables the researcher to seek theory by immersion in data transcripts in order to identify concepts and linkages that may create theoretical insight. Thus, the goal of this research was to focus on factual descriptions of events in nurse participants' own words in order to arrive at an individual exploration of moral distress perceptions and experiences-not create a theory. However, Glaser's (1978) point that upon entering a research setting the notion is to start with as few predetermined ideas as possible still applies to this study's methodology. In particular, logically deduced á priori hypotheses will taint a researcher's formulation of thoughts regarding what is actually occurring in the research setting. Specifically, when asking participants about their perceptions and experiences of moral 
distress, moral residue, stress, coping, and quality patient care, the goal was not to use prior literature and theory from these bodies of work to force participants down a particular line of thought. Instead, open-ended questions allowed participants to provide a concrete account of events in their own words from their own, unique perceptions. Remaining sensitive to the data includes recording events, non-verbal cues, words, ideas, and happenings without first having them filtered through and manipulated by preexisting hypotheses and biases (Glaser, 1978). Importantly, this qualitative study remained theoretically sensitive to the data generated by each participant. Moreover, when a participant brought up a concept or idea that was not previously considered, such as the positive aspects of moral distress, then the researcher remained open to that possibility. The nuances of qualitative interviewing techniques included not leading participants to any specific answer, but to let the data speak for itself through a clear rendering of the subjective lived experiences of the participants. Specifically, an exploration of the participants' perceptions and experiences conveyed in their own words was the focus of this study. Thus, the lead investigator maintained a journal, notes, and memos to ensure that biases and prior assumptions did not interfere with participants as they relayed their perceptions and experiences. Furthermore, in order to remain theoretically sensitive throughout this qualitative descriptive research approach, the researcher remained open to what was actually happening through the firsthand accounts of the interviewed participants. In doing so, prior crescendo effect and stress and coping theory did not inherently influence or bias the data collected from the firsthand accounts of SICU nurses.

\section{Sample}


Twenty-one ICU nurses, selected by maximum variation (heterogeneity) and snowball sampling (Patton, 2015), comprised the sample recruited from two adult trauma surgical ICUs at a regional metropolitan academic medical center until theoretical saturation occurred-where no new information was obtained (Sandelowski, 1995). To be eligible to participate in this study, registered nurses must have been: (a) proficient in English reading, writing, and speaking; and (b) employed in the two adult trauma surgical ICUs. Participants were included regardless of schedules and length of experience in the ICU. Purposive sampling is the ability to strategically select information-rich cases that illuminate a question under investigation (Patton, 2015). Maximum variation sampling was used to purposefully select a wide range of cases to highlight diversity of moral distress by sampling from two adult surgical ICUs, differing shifts, varying education levels, variable years of experience in the ICU or non-ICU setting —including different age, gender, and race/ethnicity. Snowball or chain sampling identified cases from nurses who know other nurses who could provide information-rich data. Flyers posted in ICU break rooms and bathrooms were used to elicit participation. To increase the maximum variation of the sample of participants, the researcher recruited RNs in person at the surgical ICUs during dayshift and nightshift on multiple days during the week, because full-time status nurses work three days per week. [Note: SICU nurse participants were interviewed prior to the public health pandemic of coronavirus (COVID-19)].

\section{Setting}

The nurse participants were recruited from a metropolitan academic medical center located in a metropolitan city in the Southeastern United States. The two adult trauma/surgical ICUs contain 20 beds total. Nurses working full-time, part-time, and as 
needed (PRN) in these two SICUs comprised both dayshift (0700 to 1900) and nightshift (1900 to 0700). This university hospital is a public sector academic and research facility that functions as a Level 1 Trauma Center capable of treating critically ill patients.

\section{Measures}

Baseline demographic information was gathered from participants prior to interviews, including: (a) age; (b) sex; (c) race/ethnicity; (d) nursing education; (e) number of years in nursing; (f) number of years/months experience in the ICU; and (g) number of hours worked per week (see Appendix B). An investigator-developed interview guide provided questions in the chronological sequence in which they were asked (see Appendix C). Semi-structured interviews addressed the specific aims and research questions through open-ended questions with probes to elicit in-depth perceptions and experiences of moral distress when caring for patients in the SICU. For example, questions focused on exploring nurse participants' perceptions and experiences with moral distress, moral residue, and stress and coping by asking about patient care situations that were frustrating due to potential constraints that prevented nurse participants from doing what they knew was right for the patient (see Appendix C).

\section{Procedures}

Institutional Review Board (IRB) approval was obtained from the UofL IRB and the hosting facility's ethics oversight committee. Participation was voluntary and informed consent was obtained. Participants were recruited from flyers placed in breakroom and bathroom requesting voluntary participation, and by snowballing sampling through recruited participants based on their recommendation of potential participants. Furthermore, nurses were solicited for their participation by the investigator 
appearing on the two surgical ICUs during random day and nightshift hours in order to facilitate a heterogeneous mix of participants from both units. This occurred until 21 nurses were interviewed, or until theoretical saturation occurred and no new data was uncovered. Interestingly, 20 nurses had already been recruited and interviewed, but one extra participant was interviewed because this last participant expressed interest and was not turned away. Furthermore, there was not any difficulty in recruiting participants. In fact, the investigator had difficulty scheduling interview times as participants volunteered so rapidly in a short time period.

Interviews were conducted in a location and at a time deemed appropriate by the participant. The investigator accommodated the participants' preferences for a time and location. Interviews ranged in time from approximately one hour to in-excess of 90 minutes in order to allow for enough time for participants to provide a full range of descriptions, but the length was subject to change in order to adapt to the diverse needs of the participants. For example, more or less time was allotted depending on the participants' willingness and eagerness to share their perceptions and experiences, and also the time and location was facilitated at the participant's request. Importantly, the adaptability and flexibility to time constraints was context dependent, so as not to overly tax participants or create emotional trauma. Participants received an incentive worth approximately $\$ 20.00$ gift for their participation in a single interview that included a water bottle, stress foam toy, and a pen. Interview data was reported in aggregate, transcribed, and coded with numbers, so as not to incriminate any of the participants and preserve confidentiality. In evaluating the study's rigor, participants were asked to evaluate if the investigator adequately described and interpreted their perceptions and 
experiences of moral distress and moral residue through member checking as described by Munhall (2012).

\section{Data Collection}

In-depth interviews were audio-recorded using a digital audio recorder. Informal interviews that use open-ended questions and seek descriptions and interpretations in the participant's own language are consistent with the descriptive qualitative approach. The interview guide was developed by the researcher with questions geared towards exploring nurses' perceptions and experiences with moral distress and moral residue. Specifically, nurse participants were asked about clinical situations where they were not able to do what was right, even though the nurse participants knew the correct course of action. The nurse participants were also asked how these particular clinical situations impacted their perceptions of their quality patient care and how they coped with the stress. The goal was not to lead nurse participants towards the researcher's own thoughts and ideas, but rather maintain theoretical sensitivity by allowing the nurse participants to arrive at their own conclusions with open-ended questions (see Appendix C). The researcher was immersed in the data and used the participant's language to describe and interpret their perceptions and experiences. The researcher transcribed interviews verbatim and the data were reported in aggregate. Triangulation of data as defined by (Richards \& Morse, 2013) gaining multiple perspectives was achieved by verifying the perceptions and experiences of multiple nurse participants, because they arrived at similar conclusions. Specifically, perceptions were explored from different SICU nurses regarding whether or not they had similar perceptions and experiences of moral distress, moral residue, and coping. An audit trail was maintained to ensure the rigor of the findings (Lincoln \& Guba, 1985). For 
example, a journal kept track of the researcher's bracketing and reflections. Memos logged interview times, locations, and questions asked. Transcriptions of the audiorecorded interviews were kept in word documents so that notes could be made in the margins. As part of maintaining a meticulous audit trail, audit trail categories, file types, and evidence were systematically recorded in a table format to aid in this study's data reconstruction as described by Lincoln and Guba (1985). For example, audit trail categories in the table included information such as raw data, data reduction and analysis, data reconstruction and synthesis, and process notes. Each of these categories had particular file types and evidence associated with them, which were determined by the researcher. For example, for raw data, some file types included audio-recordings and transcripts and evidence associated with these types include dialogue, descriptions of events, environments, and behavior. For data reduction and analysis, file types included write-ups of field notes, summaries of condensed notes, behaviors, ideas, and concerns notes; and theoretical notes regarding particular working hypotheses, concepts and hunches. Evidence for this category included summarized transcripts along with the results of data analysis. Data reconstruction and synthesis file types included the categorical structure of themes, along with definitions and relationships along with an interpretation of findings and potential inferences for conclusions; also a final report connecting data to existing literature. Evidence for this category includes creating hierarchies of concepts and categories, explanations of concepts, and completed documents as part of the final manuscript. In summary, process notes included file types such as methodological notes, trustworthiness notes, and audit trail notes. Evidence for these types of files ensured that data were audited meticulously. For example, in 
methodological notes, evidence such as sampling techniques, descriptions of the emerging design, and explanation of analytic strategies are available. Moreover, evidence for trustworthiness notes included peer debriefing interactions, triangulation reactions, and prolonged engagement and role. Finally, audit trail notes included evidence that displays dates, cross-references, and linkages. Furthermore, Word and Excel software saved progress as networks and matrices to analyze themes and content. For example, networks organized nurse participants' data into nodes and links to facilitate display of concepts and lists were made using matrices in order to arrive at a display of themes across all nurse participants (Miles, Huberman, \& Saldaña, 2014). Thus, another researcher may come to the same reasonable conclusion after following the journal notes, memos, transcription writings, and Microsoft Word and Excel electronic content. All of the audit trail resources were de-identified. Recruitment of ICU nurses occurred without discrimination based on age, sex, or race/ethnicity.

\section{Data and Safety Monitoring}

Interview transcriptions, journals, memos, and sensitive information were stored in a locked cabinet and password-protected computer in the investigator's nursing office. Audio-recorded data were erased after verbatim transcription and de-identified to preserve participants' confidentiality. Furthermore, an extensive audit trail was maintained along with the journal entries and memos that also included de-identified data that preserved confidentiality.

\section{Rigor and Trustworthiness of Data}

Lincoln and Guba (1985) noted four factors related to tests of rigor, including: truth value, applicability, consistency, and neutrality. Truth value is discovered by 
investigating participants, not from á priori conceptions; applicability is obtained by studying participants in their natural settings (i.e., in the surgical ICU); consistency is achieved via auditability allowing another researcher to follow the main investigator's decisional process; and neutrality is gained when the research process and product are absent bias (Lincoln \& Guba, 1985). The trustworthiness of data was ensured by credibility, transferability, dependability, and confirmability of interview data. According to Lincoln and Guba (1985), to increase credibility there must be: (1) prolonged engagement with the participant; (2) persistent observation; (3) triangulation using multiple sources; (4) peer debriefing; and (5) member checks. In credibility, prolonged engagement allows the researcher to build trust and learn about perceptions and experiences in the context of a work environment. A 60 to 90 minute interview met this requirement.

To mitigate bias from the researcher's perspective, the researcher bracketed previous experiences through journaling and reflective writing. Furthermore, to mitigate bias from the participant's perspective, the researcher encouraged openness and also reiterated the confidentiality of the interview, transcripts, and data to promote honesty that did not reflect a fear of being "found out" by management or other healthcare professionals in the facility. Persistent observation ensured that sufficient time with the participant gained meaningful descriptions of the experience of moral distress through observing the nurse's speech patterns, body language, and emphasis on words. Triangulation was the use of multiple sources to improve the likelihood that descriptions and interpretations were credible. 
Transferability was achieved through thick descriptions of moral distress and facilitated by a wide range of information using maximum variation sampling according to Patton (2015). Dependability was accomplished by the way questions and probes were asked and by maintaining notes, transcripts, and descriptions and interpretations (Lincoln \& Guba, 1985). Likewise, confirmability was established by an audit trail, triangulation of sources, and reflexive journaling. The goal was to "ascertain whether the findings are grounded in the data" (Lincoln \& Guba, 1985, p. 323). Triangulation involved recording the descriptions and interpretations from multiple sources in a journal (Munhall, 2012). Reflective writing, re-writing, and memoing in the journal immersed the researcher in this study's specific aims as described by Munhall (2012). A comprehensive audit trail was kept through meticulous recording and maintenance of notes throughout the research process (Rodgers \& Cowles, 1993).

\section{Data Analysis Plan}

Content and thematic analysis was continuous and began with the first interview, because the emergent design was an iterative process (Lincoln \& Guba, 1985). According to Miles et al. (2014), data analysis includes three parts: (1) data condensation; (2) data display; and (3) conclusion drawing and verification. Data condensation concentrated and transformed notes, journal entries, transcripts, and observations into a distilled and digestible form (Miles et al., 2014). Data display used condensed data and modeled it in matrices, tables, figures, and networks as part of content and thematic analysis. Data display was facilitated through drawing figures on a whiteboard to represent concepts, perceptions, and experiences. Conclusions were drawn and verified by analyzing and 
interpreting patterns, themes, and content so results were grounded in the data

(Krippendorff, 2013; Lincoln and Guba, 1985; Miles et al., 2014).

\section{Protection of Human Subjects}

Submission of the necessary documentation to the appropriate IRBs was done to ensure protection of human subjects by maintaining confidentiality without risk of potential harm from employer retribution. IRB approval provided participants with confidence (Munhall, 2012) that this study will build upon an existing body of moral distress research. Participants experienced a combination of risks and benefits. A benefit included the therapeutic response in acknowledging personal angst and anxiety with respect to moral distress associated with patient care. According to Epstein et al. (2016), by acknowledging moral distress, relief is the most common response and is not a sign of innate weakness or cowardice, but rather a sign of caring, competence, and advocacy. Potential risks included safeguarding audio-recorded interviews and transcripts with identified participant discussions to preserve confidentiality. Management was not interviewed because that was not the focus of the study and participants may have feared retribution. Interviews may initiate conversations about unit/team and system/organizational root causes of moral distress that may be sensitive, because they highlight specific individuals, procedures, and/or treatments.

The protection of human subjects was vital to the integrity of the research findings and the ethics of the research process. "Ethical sensitivity is a type of practical wisdom - both a resource and a process which professionals bring to, draw on, and develop in practice through self-evaluation and insight into the impact of their actions on others" (Weaver, Morse, \& Mitcham, 2008, p. 615). Confidentiality was protected using 
de-identified notes, memos, and transcriptions (Munhall, 2012). Verbatim audio recordings did not have identifying information. Probing questions were only be used if the participant was comfortable with relaying intimate details (Richards \& Morse, 2013). Participants were not pressured, manipulated, or made to feel that their response was required (Munhall, 2012).

Acknowledging the sensitive nature of ethical conversations occurring during interviews required the researcher to develop a plan for protecting participants' rights, interests, and personal integrity. Potential ethical issues SICU nurses may experience in caring for patients include: (a) threats to their professional obligations to be patient advocates that do no harm;

(b) identifying inappropriate treatments in practice to prevent harm to vulnerable patients; and (c) maintaining an ethical work environment by discussing the moral dimensions of practice and considered judgments (Epstein \& Hamric, 2009; Hamric et al., 2012). Ethical issues were recounted by participants during all interviews. A potential benefit was information-rich descriptions and subsequent interpretations of moral distress perceptions and experiences. A known risk was that nurses were asked to recount information that was viewed as immoral and traumatic. A potential risk was that interviews may have encouraged nurses to "whistle-blow" or "tattle" on their unit and coworkers. However, because risks are inevitable, this was an inconsequential point; confidentiality of all participants was maintained by the researcher. Another potential risk was that nurse participants would converse with each other about the interview experience; however, prior to each interview, the nurse participants were informed to maintain confidentiality about their personal discussions. 
The main concern in researching moral distress among ICU nurses was conjuring up negative emotions. Exploring nurses' experiences presupposes that general, openended questions are asked through semi-structured interviews (Munhall, 2012; Richards \& Morse, 2013). This created an open forum where the nurse was free to bring up whatever experiences are negative and troubling for them, which may result in response burden. The interviews did not serve as a form of therapy. If an overwhelming sense of burden occurred or was noticed from verbal and/or non-verbal cues, then the participants were given an opportunity to take a break. The response burden was never to the point that other options were required, such as the option to contact an employee assistance program and/or chaplain — which would have been provided confidentially without cost had the inquiry been made by the nurse participants. No other resources were prepared in case any nurse participant had difficulties with the process of the research. However, in the event the nurse participants became uncomfortable, or voiced aversion to particular questions, the interview would cease. The goal was to create a safe environment that was not offensive or manipulative, and researchers provided information that assisted the employee. 


\section{CHAPTER 4 RESULTS}

\section{Participants}

The sample consisted of 21 nurse participants who worked in the Surgical Intensive Care Unit (SICU): 15 were female, six were male, and one ICU float-nurse. The average age of the sample was 34 years of age. The mean number of years worked in the SICU was 6.7 years. The mean number of hours worked per week in the primary SICU was 35 hours ( $S D \pm 7.7$ hours). The sample was primarily Caucasian.

Nurses from two SICUs at a large, level-1 trauma academic medical center were interviewed. Both SICUs were similar: 10-bed units staffed with five nurses, a respiratory therapist, secretary, and a medical assistant from 0700 to 1900 dayshift; and five nurses, a respiratory therapist, and a medical assistant from 1900 to 0700 . Nineteen participants reported that they socialized with friends and co-workers from the hospitals where they worked. Interestingly, participants claimed to do a multitude of activities to relax. For example, their activities included playing golf, eating, television, reading, walking, exercise, gardening, video games, going to the gym, yoga, running, cycling, and spending time with friends and families.

\section{Overview of Moral Distress by Nurses in the SICU}

The purposes of this descriptive qualitative study was to explore: (a) SICU nurses' perceptions and experiences of moral distress and moral residue in the adult SICU environment in order to determine root causes of moral distress; (b) nurses' 
perceptions and experiences associated with moral distress, moral residue, stress and coping, and perceived quality of patient care. By exploring these concepts among SICU nurses, the researcher can identify moral issues that lead to moral distress. From these results, it is hoped that a better understanding of the phenomenon will be achieved leading to insights essential to the development of future interventions to address moral distress and moral residue among SICU nurses and improve the quality of patient care, in the SICU environment.

First, it is important to discuss the fidelity of the interview process, because fostering an interpersonal milieu facilitated genuine and thoughtful responses. A particular level of trust and rapport was required for the participants to provide such emotional and powerful personal accounts related to nursing in the SICU environment. Specifically, preliminary introductions were not necessary and minimized the time required to establish a trusting relationship. All 21 nurses were interviewed individually and in a private setting away from the SICU. Interviews ranged in time from 45 minutes to over two hours. Participants stated that discussing their perceptions and experiences with moral distress and moral residue in the SICU was helpful. In particular, participants were able to recall specific dates, times, patients, names, and events in great detail when asked to recount a patient care event where they were not able to "do the right thing" for the patient because of circumstances out of their control.

Moral residue occurs when situations that are morally distressing occur repeatedly and are not resolved (Epstein \& Hamric, 2009), which creates nurses who remove themselves from the ethical dimensions of practice through compartmentalization. To understand the moral distress among the nurses in the SICU, it is important to aggregate 
their individual stories to understand the underlying process. This development of moral distress blatantly calls into question the moral integrity of the nurses' practice because the nurses are not able to "do the right thing" for the patient either through their action or inaction. That is, the nurse is not able to "do the right thing" because of limitations that are broadly perpetuated at the organizational systems-level, such as staffing decisions and resources directed at managing the staffing of various healthcare disciplines throughout the organization. Based on the results of this study, these systems-level limitations were manifested as situations causing moral distress as a result of short-staffing, poor communication, imposed decision-making through power differentials (e.g., MD to RN), and cases of futile and/or aggressive treatment regarding patients with poor prognoses and at end-of-life. In addition, participants readily recounted past events with specific times, dates, and were able to recall and discuss particular details. The events were ingrained in the participants' memories. Over time moral distress may result in positive coping and/or some maladaptive coping behaviors. However, it is difficult to assign blame to some maladaptive coping after listening to the stories relayed by the participants.

Importantly, it is necessary to understand the process these interviews have revealed about the development of moral distress and moral residue among nurses in the SICU setting, as follows: 1) some aspect of patient care creates a morally distressing scenario; 2) the SICU environment where the event takes place has numerous influences that must be considered as part of the process leading to the nurse's experience of moral distress; 3) moral distress is perceived and experienced through some specific clinical experience; 4) left unresolved, moral distress remains and seethes underneath the surface 
of the nurse's psyche through the stain of moral residue - meaning that the nurse may likely experience future similarly morally distressing events and that there may still be unanswered questions that prevent the nurse from arriving at a solution- - thus, nurses may be emotionally upset about the way situations at work are handled and become stressed, anxious, or depressed, and may cope through drinking, drug use, smoking, overeating, and exercise; and 5) eventually a moral crescendo occurs where the clinical situation at that particular moment is too much or the ongoing bombardment of circumstances has resulted in a call to action on the part of the experiencing nurse - this final step is the coup de grâce that is the result of unaddressed moral distress.

A repeating theme from the research process was concern for the well-being of the participant - the nurse. This well-being was primarily concerned not only with some of the participants' manners of coping with the stress, but also how participants handled morally distressing moments. For example, some participants expressed regret, selfcriticism, anxiety, depression, and trauma from their experiences of moral distress. Thus, a concern for the nurses' well-being was noted as part of the data collection process and presentation of the results with a particular effort to maintain the participants' confidentiality.

Five themes of moral distress were identified from the interviews with SICU nurse participants in this study: (A) Theme 1 - Concentrated Caring in High Stress Environment- “Doing Your Best" was comprised of the following sub-themes: (a) "doing your best" for the patient; (b) caring; (c) communicating with families of patients near the end-of-life; and (d) suffering and inner turmoil; (B) Theme 2 - Advocating for Patients \& "Doing the Right Thing" was comprised of the following sub-themes: (a) 
physician-nurse power differential; (b) patient advocacy; and (c) positive nursing "doing the right thing"; (C) Theme 3 - Surviving Moral Distress in the SICU Environment-Magnifying Stress \& Self-Preservation with the following sub-themes: (a) nursing management problems, short staffing, and lack of resources; (b) incompetent RNs lacking in SICU experience; (c) demands of additional charting and responsibilities; (D) Theme 4 - The Stain of Moral Residue-Surviving \& Coping with the Impact on Integrity \& Values, with following sub-themes: (a) surviving and coping with the impact on personal moral integrity and values; (b) compartmentalization; and (c) nurses “just punching clock"; and (E) Theme 5 - Moral Crescendo's Coup De Grâce-Suffering and Wounded: "Cannot Take It Anymore" with two sub-themes: (a) burnout; and (b) "cannot take it anymore - ready to leave".

Conceptualized within each individual story is a root cause of moral distress, which is subsequently connected, and part of a broader process. This process of moral distress leads back to a system-level issue, that if resolved, then there is less distress among nurses, as well as more quality approaches to patient care in the SICU may be achieved.

Importantly, as the themes of moral distress were identified, it is necessary to recall the formula for the process of developing moral distress by the following equation, as follows:

Nurses' patient care experience + SICU environment $=$ Moral distress (single event) $\rightarrow$

Moral residue (left unaddressed, accumulates) $\rightarrow$ Moral crescendo (quit, burnout, PTSD) 
For ease of interpretation, simple models of moral distress were developed to represent the complex, nuanced clinical situations in the SICU environment that led to negative experiences of moral distress for nurse participants. Thus, bracketed within this equation above are the following assumptions:

1. Complexity of SICU patients and stressful environment $=$ This SICU environment creates stress for the nurse and the nurse has an initial response [positive, negative, or even neutral at the time that events are unfolding].

2. Complexity of providing nursing care for SICU patients $=$ STRESS accumulates over time as initial responses are not resolved, creating moral residue that stains the SICU nurses' experiences and perceptions.

3. Complexity of the SICU environment and inability to establish an outlet over time $=$ Moral Crescendo. It is noteworthy that this moral crescendo occurred only in a few cases in this study. For the most part the nurses were hopeful because they had other "outlets" for stress as they were in school and not "burned out". However, other SICU nurses perhaps were more isolated in their anxiety, except for the companionship of fellow co-workers who understood.

Nurse participants recounted their experiences couched in a context of always wanting to "do their best" for the patient's care. Interestingly, each nurse participant was at a different point in the development of moral distress identified in this study. Some participants had more experience than other participants, as well as differences in age and educational preparation. Additionally, a few participants reached the endpoint of the process - moral crescendo.

Theme 1 - Concentrated Caring in High Stress Environment—-Doing Your Best" 
Concentrated caring was identified as the first theme because so many participants expressed this as the impetus for evaluating the quality of their patient care and the responses centered on "doing their best" for the patient. This theme highlighted the first part of the process model of moral distress, which describes the complexity of SICU patients and the stress involved in providing nursing care. While the impetus for moral distress involved these complicated patients and stressful situations, the participants always were focused on the patient and the quality of care. The patient was the focus despite the maelstrom of events occurring around each nurse participant. Their responses were couched in the context of caring. Nurse participants focused on how they were able to make that patient's particular hospitalization the best experience as possible for the patient despite the circumstances.

Theme 1: Concentrated Caring in High Stress Environment—“Doing Your Best" represents the complexity of SICU patients and stress involved in providing nursing care, as well as what encompasses the best about patient care. This theme highlighted the first part of the model of moral distress, which is the complexity of the SICU environment, patients, and the associated stress. Concentrated Caring in High Stress Environment"Doing Your Best" is comprised of several sub-themes and includes: (a) "doing your best" for the patient; (b) caring; (c) communicating with families of patients near the endof-life; and (d) suffering and inner turmoil. Importantly, nurse participants described caring as when they were doing their best for their patients. Furthermore, communication with families was viewed as difficult, but a necessary part of the SICU role of the nurse. For example, some participants were "stressed out" by family communication, while other participants found it necessary and therapeutic. The majority of participants 
revealed being morally distressed about clinical situations that involved end-of-life, suffering, and the inner turmoil involved. Often in these cases, participants felt that physicians did not do an adequate job of communicating with families about the reality of the patient's prognosis, although the nurses noted that this is often difficult with multiple physicians and medical specialties for each particular patient.

\section{1.a. Doing Your Best for the Patient}

All nurse participants agreed that their personal indicator of providing quality patient care centered on the notion that they were "doing their best" for the patient. This provided many participants the rationale to keep moving forward. For example, some participants were frank about their experiences. Number 2 nurse said the following: "So I guess the way I cope is, I... first I validate myself through, did I do my very best”? (\#2, p. 44) Number 1 nurse described doing her best as the following: "Felt care was good - in the capacity I had I did everything I could" (\#1, p. 3). Nurse participants wanted to ensure that patients had the best experience possible. Number 7 nurse described his patient care in the following way:

I think I still, I still feel an obligation like, to do the job that I'm paid for. And I try to stick by that. You know? You know, because they pay to put up with a certain level of bulls--t and that's just what you have to do. And like it's, at the end of the day, it's customer service. It's like, I mean, I would say it's probably like the highest form of customer service you can have because you're dealing with like all their essential functions really. Half the time we're breathing for them. (\#7, p. $37-38)$

Number 8 nurse, said the following about doing their best: 
Really, I mean me per say, I feel like I do the best I can every time I'm there. It might not be the same care you give or you know like other people on our unit that are better or smarter than me or worse, you know, I like, I'm doing the best I can. I can't expect to do any more than that. But I do think interdisciplinary cooperation can affect it, you know, if I'm not getting the return page from the doctor quick enough to change orders or physical therapy comes by and they're sleeping and then leave, that can overall affect my care, which may mean getting this person up to a chair would really benefit them, but I can't get any pain medicine ordered, and therapy doesn't have time to wait. (\#8, p. 7)

One nurse (number 4) prided herself on her ability to recognize errors from pharmacy and by reporting them she was doing her best to make patient care safer. This nurse described her experience as follows: “Oh, it's surprising. It's surprising. There's all kinds of med errors. I find more pharmacy errors, dose errors, time errors-everything" (\#4, p. 22).

Number 7 nurse described not feeling confident about how much experience he had to take care of SICU patients. However, he noted that he was "doing his best" because he wanted to take care of sick patients and advocate for them, as follows: I think it's part of the learning process. Like school is not going to teach you everything, when you get started at [name of hospital organization] you're not going to know everything. And you know, you're just going to keep working until you learn more, you know, or because you're still learning the job even if you've already been there a year. But it's like people look at you like you know 
everything and anything about either their patient or their diagnosis. And you don't, you know? (\#7, p. 28)

Thus, this nurse's perception is that as everyone looks at him, he was recognized as doing his best even though he may not feel like it. Even though this nurse was not confident in his ability to function as a nurse, he was still trying, which is the attitude these SICU nurses needed to survive the clinical environment of the SICU.

Nurse participants discussed wanting to provide patients everything they could in the 12 hours they were working in the SICU caring for the patient. Participants discussed setting the bar high for themselves and working hard while they were in that environment. One participant (number 5) had this to say about doing her best:

If you do your very best and if you know you're doing the best you possibly can on that day um and something happens you did your best what else could you do? And a lot of 'em, like I don't know it's not my place to judge you, it's just my place to heal you while you're in the hospital. $(\# 5$, p. 22)

Participants revealed that their focus is on the patient and providing the best possible care for the patient. Some participants feel that they are inexperienced because they have not worked in the SICU for a long time. Participants who were more experienced felt that it was their duty to care for the patient, work hard, look for mistakes, and have situational awareness of their surroundings.

Nurse participants mentioned when they are at work, they are "present" and work hard. Moreover, even in the face of angry and abusive patients and/or families, participants strove to treat everyone the same - with respect and kindness. Interestingly, 
one nurse (number 10) loved the patient care aspect of her job, but complained that the administrative part was difficult:

Absolutely because you also don't, I mean, not only do you not want to have anyone feel neglected, you want to make sure, like I said, you want to make sure everyone gets the same level of care, but you also know, like, how things happen; I mean you don't want to pass your little stuff onto the next shift. Because that little stuff adds up and ends up being big stuff. (\#10, p. 12)

This nurse went on to say that:

“... I feel like I'm responsible for kind of like helping to do and fix the things that other people aren't doing." (\#10, p. 23)

Interestingly, one nurse (number 13) who was very stressed from the job working in the SICU said the following: "I care about everybody and I feel every patient needs to be treated with dignity and respect, regardless of what they're there for, or who they are" (\#13, p. 5). Furthermore, nurse (number 12) had this to say about patient care: "So it's kind of like we've learned to just make it through the night and try and do the best we can" (\#12, p. 3). The majority of participants noted that doing his or her best for the patient was the ultimate form of caring. The participants consciously verified that they are doing everything possible within their scope of practice for the patient.

\section{1.b. Caring}

Caring was repeatedly named by nurse participants as a method of evaluation. Numerous participants were focused on caring and discussed moral distress in the context of the caring for the patient. For example, nurse number 7 in particular said she was 
"burned out" because she cared so much and was bothered by nurses who did not care as much as she:

So to me, there's no, it's not that there's no difference, but to me that's still and like I wouldn't change the way I care about a patient based on it 'cause like my job's not to necessarily pass judgment, but still to care... (\#7, p. 5)

One nurse participant noted that she treated patients like "her family" in order to maintain the same caring attitude. Specifically, nurse number 5 was caring for a patient who used methadone regularly and felt that it was her duty to treat her like a human being:

She's like, 'I just think that they look different at me because I take methadone every day.' And I wouldn't want my family to, like, say my family member has drug problems. I wouldn't want him or her to be treated any differently than you or I. (\#5, p. 16)

Other participants said that providing the best care was their way of doing their best for the patient. For example, number 8 said the following:

Patients are all strangers to me. I'm not emotionally connected to them-in their lives, but somebody is, that's someone's loved one. They deserve to get the best care they can and get out of the hospital as quick as they can. (\#8, p. 13)

Another participant felt that if she was able to validate herself by evaluating whether or not she "did right" by the patient in staving off negative patient outcomes:

Did I do my best? And if I did my best, there's nothing to cope with. Even if you lose a patient. if you did your very best, if you did everything in your power to do right by that human being, then there's really not much to cope with. Like, yeah, I'll come home, I'll cry a little bit, but I did my best. (\#2, p. 44) 
Another nurse participant (number 11) reported:

I care more because then I know more and I feel like I should show that I know more. It's funny because like when I learn something new then I'm like, 'oh I need to apply that at work'. I just learned something new the other day about how to put a foley in better, easier, and I was just like how do we not know... (\#11, p. 24-25)

The assertive nature of SICU nurses was exemplified by the way they concentrated their care for the patient. This particular nurse (number 2) demonstrated great communication skills and was assertive about her point, while also being emotional about patients. She would visit her patients once discharged from the SICU when they were in rehabilitation units in order to see how they were progressing:

And we're ICU nurses. If we don't speak-up for people. Yeah. If ICU nurses weren't the loudest in the hospital—nothing gets done. We're the ones that we have more autonomy than other nurses in other areas of the hospital and I think it's very, it's because we need it. And it's because we understand our role a little bit better. And it depends on the team and the time of year and everything, but like there are times when I will definitely ask for forgiveness later. Rather than beg for the ability to do something. I mean sometimes you just have to trust your gut and if I can't if my gut tells me something like in in my patient's case, my gut told me that he did not need that surgery, um, I wish I would've spoken up for him. [cries]. (\#2, p. 17-18)

Nurse participants readily expressed kindness to the patients for which they were caring. For example, participants noted the difficulty of caring for patients' pain for those 
with addiction problems. Nurse participants noted the imperative to treat these patients the same and with respect and kindness. This was difficult for some participants, particularly when the patients were aggressive, mean, and cursed at the nurses. Nurse participants discussed the importance of working with a supportive group of nurses to communicate with and make the shift better in the SICU. For example, one participant bemoaned that you cannot achieve the desired outcome all the time, but as long as patients feel like they are being treated the best, then the care is justified: "So as long as we can, for me, as long as my patients feel like they've had the best experience they possibly can, I feel like we've done good. Even if their outcomes aren't ideal” (\#2, p. 43).

\section{1.c. Communicating with Families of Patients Near the End-of-Life}

Communicating with families was found to be both positive and negative for some nurse participants. Numerous instances of family communication occurred within an end-of-life context. However, regardless of how participants felt about the process of communication with families, it was still a necessary component of their job. Participants would enlist the help of the patient's family to facilitate patient care and help with processing of emotions. For example, a particular nurse was in-tune emotionally and situationally-aware of how communication is necessary for the job. In particular, she highlighted the importance of doing her best and caring for the patient in a context of the family as being an extension of the patient. However, this particular example took place in a context of end-of-life / suffering because the healthcare team went against the patient's advanced directive treatment preferences by extending the life of the individual:

It's hard because you're primary person that you're caring for is that patient. The person in the bed is your number one concern. Now if you feel like there's not a 
person left there, if you feel like it's just essentially a bag of meat that you're caring for because their brain is gone, then your focus kind of shifts to the family. Once I had eliminated him as like a person with their own wants and needs and desires, then my goal shifts to providing care for the family and now if the family has chosen to ignore an advanced directive. It's what they can live with. So, the way I justify in my head: if you can't care for the family—or the patient—-then you care for their family. Because you have total care in my opinion and the way I try to practice is that the family is just an extension of your patient [crying]... sorry. And sometimes it's really hard and I feel like other nurses don't always value the families. I think it's just difference of personality. And a difference of experience. (\#2, p. 3-4)

Interestingly, this quote captured that this nurse participant felt that a co-worker in particular did not care for families and extend the same courtesy of caring for the patient to the family as the central unit of concern. Many nurse participants discussed building relationships with patient's families as way to build rapport and relieve stress. Nurse 7 used the following method to communicate with families:

Difficult to communicate to the lay community about the permanency of neurologic damage, because they do not understand it. The family does not understand the finality of their loved one's diagnosis from a traumatic brain injury. Or thinking like that, but I don't really approach the family as far as what's your long term goals, you know, I'm more of a supporter of whatever they think is going on at the moment, or if they ask me a question, I try to give you the most 
realistic answer I can give them without being kind of overall, I guess, overwhelming, or really gloomy. (\#7., p. 5-6)

Interestingly, communicating with families was a way for nurse participants to show that even though the job is stressful, complex, and fast-paced in the SICU, they should still be intentional and deliberate with their practice in order to advocate for the well-being of their patient and the understanding of the family and loved ones involved in patient-centered care. One nurse participant (number 7) discussed working with residents physicians who would give family "false hope" by scoring the patient with a falsely high Glasgow Coma Score. For example, this particular participant noted that the patient had not localized to painful stimulus in weeks, yet the neurosurgery team told the family that the patient was localizing, but the nurse participant expertly noted that the patient was not able to approach midline with either upper extremity:

I think, I think that was the issue and what I learned from that first case, like we kept doing these neuro exams and they're all just kind of giving these like not so accurate exams, which kind of kept the family on the line as far as keeping her, you know, keeping her around and I just don't think that was you know, it was kinda irritating. I mean, cause, it just wasn't correct. Like they would give her higher scores on her Glasgow Coma and I was just like that's just not accurate, like they tried to say she was like localizing at one point and I was like she hasn't localized in like weeks. (\#7, p. 12)

Another nurse (17) revealed that trust is important when communicating; however, it is also important for the nurse to demonstrate awareness that not every 
question ought to be asked because it may offend or impact the establishment of a trusting relationship:

I guess the way I look at things is, you know, I take people's, like, trust and transparency very seriously, like, even from the time I get an admission history, you know, families will be like, you know, when you ask the questions of like, the drug and alcohol stuff, but like I always ask like you know the things I feel like I need to know and I feel like that's like a very hyper-personal question not only that, but like your medical history like you know whether that be that you know something that like is more taboo or maybe you have like something normal like congestive heart failure, but like to me, I feel like that takes, like, takes a great deal of trust to tell someone everything about you. (\#17, p. 6)

This same nurse (17) revealed that she works on her perspective when initially communicating and establishing trust with families in the SICU environment:

I take that trust and transparency very seriously and I always ask for that same trust from the family members I take care of, like, when they're grilling me, or questioning me, I try not to, like, take that as a personal measure, but rather as like, you know, that they're trusting me and they want to know all the things that I know. I feel like all humans want to be seen, heard, and valued. Like those three things and when you can't give people those things - you can't, you lose their trust. And once you've lost that - rebuilding that in a hospital-stay is nearly impossible. It may be minimal to you. So that's why, like, I try to treat it and I try to react in a way that shows respect and like reverence to the families' feelings maybe they think like this car accident is like no big deal and it's no big deal to 
them and they're going to get better, so I try to like not interfere with like people's emotions. (\#17, p. 7)

Another nurse participant (19) noted the following frustration when patients become incapacitated and families make decisions that do not reflect the patient's preferences:

Well, and then there's every single time someone codes and they didn't want to be a, you know, they didn't want to be a full code, and they said they didn't want to be a full code, but the family, once they're unconscious, changes it. And that happens way too often. (\#19, p. 4)

One nurse described her experience as "burnout". She is ready for a change where the clinical environment is less stressful, anxiety-provoking, and depressing. For example, this participant exemplified family communication. She described how, in situations of short-staffing, communication with the family may be difficult, but she also thoroughly enjoys communicating with the family and extending their partnership in the patient-family centered care experience:

Acuity-wise this person should be a one-to-one. But we don't have the staff to do a one-to-one, so you're still responsible for another patient. It sucks because then you go in there like 3 hours later and you're like, 'you doing okay? I haven't seen you in a while.' And then sometimes, like especially family members, like it was somebody that maybe they're on the vent but they're not like completely with it, but they're super stable and kind of ignorable. But they have family members. The family is like, 'where the heck have you been? They were coughing and they needed suctioning and no one came in.' And you have to just like apologize and 
say, 'well I just couldn't make it in here.' And so they just don't get the true care that they need because you still have a team, but you're trying to keep this other one alive because they're more unstable. Yeah, I mean, I can't do anything about it, but I feel like if there was more like an additional hand to help me I could say, 'hey, can you just go check on them and make sure they're okay and make sure they don't need anything?' Versus when you don't have that additional hand it's like going to be a couple hours, so hopefully they're still kicking. (\#6, p. 12-13) When viewed in the context of communicating with families, this particular quote adequately captures a whole confluence of circumstances in the SICU. In particular, this nurse described the short staffing experienced and how that impacts family communication, but she also discussed the importance of extending partnership in one's care with the healthcare team, via the nurse, and family.

\section{1.d. Suffering and Inner Turmoil}

All nurse participants described some degree of suffering on the part of the patient and the experience of inner turmoil on the part of the nurse. Interestingly, all of the morally distressing clinical situations were the result of circumstances contextualized around the end-of-life, or a decision to continue treatment on behalf of either the family or healthcare team. Regardless, in all instances, the nurse participants perceived and experienced inner turmoil, because they were concentrated on providing care that was in the patient's best interests. The nurses believed that the patient's suffering was often the reason behind their experience of moral distress. For example, this nurse participant recounted an experience that caused inner turmoil about advanced directives and family decisions to treat regardless. Moreover, this participant captured the clinical situation and 
subsequent inner turmoil from the nurse's perspective, particularly as she revealed the patient's suffering:

He was in a bicycle accident riding. He like decimated a large chunk of his brain. His wife happened to be a nurse and wanted us to do all the things now he had an advanced directive that said that he did not want to be trached or PEGed - he didn't want tube feeds anything like that. But in Kentucky we frequently don't honor advanced directives - we go by family wishes and she chose to trach and PEG this man. At the time I'd seen his scans, he'd had a stroke in every single part of his brain. They had to do a washout. He had a crani and they did a washout. They removed a significant portion of his temporal lobe because there was so much debris inside of it. I didn't see the value in a trach and PEG for him especially because it did not go with his wishes, so we trached him and we PEG'ed him and we sent him off to uh Frazier Rehab even though he was 5 to $7 \mathrm{~T}$ on the Glasgow Coma Scale. We went to go visit and just say hi and show the family that we're still supportive even though they're not our patients anymore. And he was awake answering questions and purposefully reaching for things; three months later he was reading to his child; six months later he was walking and talking and having somewhat meaningful conversations with his family. So it would just this like vacillation of we shouldn't do this to holy crap how many people have we killed that had a potential? [laughs]. He had had a stroke in every part of his brain. We had removed parts of his brain - he had had massive infections already just through like being orally intubated and through like all of that - like he had setback after setback with his recovery experience. And then 
when she elected to trach and PEG him, there's not a person in that unit - not a single person that thought there was any reason that he should go through that. Especially because it was against his wishes. I took care of him admission to discharge. (\#2, p. 1-3)

One nurse participant bemoaned that American medicine "saves everyone" and contended that not everyone needed to be saved due to the age of the patient, severity of the traumatic injury, and projected quality of life/long-term prognosis, as follows:

People who are run over by dump trucks should not be saved. This is wrong. And that's part of the problem. You can't have 80-year-old people who have been run over by dump trucks with their entire intestines hanging out and they're surviving. Who intubated these people to begin with? And you have to like let that go. Yeah. You have to just let it go. You can't. I mean these people shouldn't have been saved to begin with - that's part of the problem. American medicine saves everybody. EMT's save everybody. Paramedics save everybody. Some of them shouldn't be saved. (\#4, p, 5-6).

Another nurse participant noted the idea of hanging onto people for longer periods of time because of not necessarily knowing whether or not they will recover. Participants noted that healthcare is a business and feel that sometimes their involvement in certain procedures is often for the benefit of the physician and their learning process and not necessarily for the family and what the patient wants.

One nurse (number 19) noted the turmoil experienced when a patient confronted her about his mistreatment: 
He was an MVA. I think he had some deficits as far as... I don't know, if it's, you know, mental or physical as far as handicap goes. Um. And now this is hearsay. Um. Because I haven't taken care of him, but I've heard from multiple sources that he had, he was a... Neurosurgery did... they saw like an osteophyte on his Cspine and so they went to remove it and ended up paralyzing him. They didn't look at an old C.T. scan to show that it was an old osteophyte that was basically chronic and benign and this, that, and the other, but also prior to his surgery he stated to the physicians and to the nurse, nurses that he did not want to be trached. And. So they trached him. And then when a certain nurse who was there, present, when he stated that, came back from her few days off, he looked right at her and said, 'Why did you let them do that?' And now every shift to everyone who walks in the room he says, 'please take it out, please take it out, please let me go.' Everyone who walks in that room-and now he's a quad. (\#19, p. 3-4) In closing, the same nurse noted the suffering a patient had to experience and the inner turmoil the nurse participant experienced as a result, as follows:

The most recent one we admitted a lady, um, suicide attempt—not her first try. She was pretty successful. Trauma physicians were the only ones on-board, because you know it wasn't a head injury. I'm not sure what the conversation with the family was but the very next day they trached her. And then later that same day withdrew care on her. So she is now still alive as of my last update. Quote unquote. A) It was an unnecessary procedure. B) It would not benefit her outcome. It would only prolong her... misery. I helped admit her. And then-Monday morning. And Tuesday I was off work. But I asked another nurse for an update. 
And they said they trached her and withdrew care on her on the same day. It goes against, you know, every reason we got into nursing which is to do what's best for the patient. And not a lot of people like to realize there's worse things than death. It's been my mantra as well as my favorite line from Jurassic Park thanks to Jeff Goldblum where he says 'just because we could, doesn't mean we should'. So. $(\# 19$, p. 2-3)

\section{Theme 2 - Patient Advocacy \& Positivity About Nursing - "Doing the Right Thing"}

Patient advocacy was identified as part of this theme because the nurse participants' moral distress stemmed from issues concerning the care of the patient. In addition, the actions of the nurse participants were of patient advocates even when they could not do what was right for the patient because they were prevented — at least they knew the right thing that needed to be done. Moreover, the MD-RN power differential was a topic that came up frequently in discussions of how power was used to manipulate through communication and decision-making. The positive nursing part of the theme has a dual meaning. For example, nurse participants were genuinely hopeful and maintained a positive outlook about nursing. However, a lot of the nurse participants were concurrently in graduate school while working full-time in the SICU because they did not want to stay at the beside. Due to the positivity among nurses interviewed, in particular, nurse participants focused on "doing the right thing". For example, issues of moral distress that the nurse participants recounted were named because they recognized that something was occurring that was not morally right in the patient's care. Therefore, the positivity was highlighted because it was not expected in this particular context of moral distress. This positivity about nursing was further corroborated by each nurse speaking highly of other 
supportive co-workers in the SICU. This optimism was inspiring and reinvigorated an excitement for the job and the work involved for the nurse participants. This in no way diminishes the nurse participant's moral distress involved in patient care, but it is important to highlight each nurse participant's survivalist perspective. It was this ability to self-persevere that allowed these nurse participants to keep functioning and moving forward.

The Theme 2 -Advocating for Patients \& "Doing the Right Thing” represents the nurse participants' response to the complexity and severity of SICU patients and the stress associated with providing nursing care, and the nurses' role advocating for patients while maintaining positivity. The majority of nurse participants discussed moral distress stemming from patient advocacy issues concerning the care of the patient. The actions of the nurse participants were as patient advocates even when they could not do what was right for the patient because they were prevented by moral distress - however, the nurse knew the "right thing" that needed to be done. Moreover, the physician-nurse power differential was frequently discussed regarding how individuals in positions of power used this leverage to influence communication and decision-making. The positive nursing part of the theme has a dual meaning. Specifically, nurse participants were candid, optimistic, and maintained a positive outlook even when they were overwhelmed with the stress and complexity of patient care in the SICU. Nurse participants concentrated on "doing the right thing," highlighting their positivity about nursing. For example, issues of moral distress were identified because nurses recognized that something that was occurring in the patient's care was not "morally right". Theme 2 is comprised of several sub-themes including: (a) physician-nurse power differential; (b) patient advocacy; and 
(c) positive nursing "doing the right thing". The physician-nurse power differential was identified because physicians used this power differential as leverage through interactions with family members, while the nurse participants recognized this manipulation and power differential between physicians and nurses. Thus, the nurse participants recognized the need for patient advocacy and the necessity of advocating for their patients with families and physicians.

\section{2.a. Physician-Nurse Power Differential}

Dealing with the power dynamic between physicians and nurses was described by many SICU nurses. Nurse participants were acutely aware of the power dynamic between physicians and nurses. One nurse participant noted that she deferred to physicians because they were a "higher authority". Other nurse participants who were charge nurses pushed back against the physician's authority and questioned orders and the rationale behind certain treatment methods. Nurse participants discussed dealing with attending physicians and resident physicians who are learning in a teaching hospital. While this environment is good for physician learning, the nurse participants discussed the difficulty at the end-of-life with regard to communicating with physicians.

The following quote by a nurse participant exemplifies her frustration with resident physicians using the MD-RN power differential to their advantage. For example, in this case the nurse participant observed that the resident neurosurgeon decided to proceed with drilling a hole in a patient's head to place an external ventricular drain (EVD) without pain or sedation medicine because the patient was hypotensive. The nurse for the patient was new and the nurse participant recognized the power differential 
manipulated by the resident neurosurgeon and the inability of the new nurse to be a patient advocate:

It makes me, like, scared for the patient just because you have some doctor that's doing just whatever she wants because she can and nobody's really overseeing her at night, like physically, and then it also the nurses that's new, I don't know maybe they were scared or they didn't know what questions to ask, I don't know, I mean it just makes you scared for the patient and kind of makes you feel bad for the new nurse because, I would have called house. Or I would have called someone else, her upper, or anybody else. That's wrong, I mean, I would've been like, 'I do not, I do not want to be a part of this procedure that you're doing, and I refuse to be in here.' But then it doesn't matter, because they went about it. And the, and the attending said that she [the neurosurgeon resident] didn't do anything wrong. (\#20, p. 7-8)

Interestingly, the following quote is from a nurse (number 13) who actually experienced a moral crescendo. However, the physician-nurse power differential may not be so blatantly obvious. Specifically, physicians may not respond readily, because of lack of concern, which perpetuates the power differential relationship among nurses. In the following example, the nurse questioned her own knowledge, because the physician was not responsive to her assessment that the patient was in need of an intervention:

It took a while for the physician to even act on that, until, I mean that's something, the physician had been up there had also seen the blood pressure and also just kind of walked in the room, explained to the family what was happening and then walked out. So, I think it was a lack on both parties. This one, actually 
because it's the most recent. It makes me feel like I have to double-think what I'm doing instead of just being able to go out there and do what I do, like I have to think more in-depth, so my critical thinking skills I feel like are kind of lacking like, you know, what I would have done differently. (\#13, p. 1-2)

From this quote, it is evident that the patient was not getting the care needed because the physician was not acting and was not responsive to the nurse's assessment and observations. Therefore, the power differential may have been used to manipulate the nurse by creating an environment where inaction is the concern despite closing the communication-loop regarding the medical issue. This nurse participant went on to say the following about physicians describing the MD-RN power differential:

"It [laughs] makes me feel like my job isn’t as important as the physician, that they rule the hospital, that what they say goes, and if they don't feel like it's important, they just shrug it off." (\#13, p. 9)

Nurse participants discussed communication occurring in a variety of directions, such as between the nurse and physician; nurse and other nurses; nurse and other healthcare team members; nurse and the family; and nurse and the patient. However, there were perils of power plays noted by nurse participants because places of leadership allowed for instances where the physician either communicated harshly with the nurse or coerced the family into an action. Nurse participants noted that communication problems between physicians and nurses were problematic because they created a barrier to providing care for patients. For example, nurse participants noted that in working nightshift, an issue that arose with communication occurred when the nightshift resident physician covered for the primary medical team. A nurse who had been a nurse less than 
two years described the following frustrations in working with physicians on nightshift and feeling powerless:

I admit this patient at $8 \mathrm{pm}-\mathrm{I}$ just started my shift. So, I get this patient from the OR. They had like two IVs for access. One of them is barely working. I'm calling trauma. Trauma's not the one that's on [call]. They're just doing the critical care on this patient. And I'm calling them. When you have a specialty like a colorectal patient, or an ENT patient - they know everything about this patient during the day. When you come to nights and you're talking to the trauma doctor, they don't really know much about 'em. They just get a quick, little sign-out. They don't know what surgery was performed that day, they have to go look in the chart and you feel sort of powerless, because you don't really know what they want, because they don't know what they want, because it's not their primary team. Trauma is not their primary team and they're just doing the critical care and say they make a decision that night, because they have to make the call, because their primary team's at home sleeping until 5 am the next morning. I think it depends on the provider, because this ENT patient is being seen by neuroanesthesia or their critical care. And then you're just calling neuroanesthesia and they're like, 'well, I don't know if this is what they want, but this is what we'll do.' And I think it's not be the best thing for the patient. (\#5, p. 9)

This situation described above created issues in patient care because nurse participants had to spend too much time communicating patient information and updating the on-call physician on the plan of care and patient's history, while also trying to save the patient's life and not being able to leave the patient's room. 
Nurse participants noted being nervous communicating with physicians and families because of lack of confidence or knowledge or experience, as described by another nurse:

I think it's bothersome, because we're not giving the patients the quality of care that they're, you know, when you come into a hospital, you know? I mean the family, when the family got there they kept asking, 'well, you know what kind of procedure?' And, I mean, they could tell them what kind of procedure, but they couldn't tell them exactly what was done, because, or like that there were any complications, or this, that, and the other, because they weren't in there and they literally did not know. How hopeless would you feel if the doctor that's coming in to take care of you, or your loved one says, 'well, we, you know, I'm the doctor that's taking care of him tonight, but you know I don't know what happened in the O.R. yesterday, because I wasn't in there, or my team wasn't in there?' (\#7, p. 1011)

This particular power differential dealt with the responsible party, the physician, who was responsible for being on-call for a house full of critically ill patients:

There are certain situations where I feel like certain patients don't get the care that they deserve - especially working nightshift. So, a lot of times when you work nightshift you're probably familiar with it, you have like your float team doctors who are not like the primary care so, basically, it seems like patients get 12 hour care at times in the SICU. For many of the float doctors, what they've said themselves, is their primary goal is to keep the patient just alive overnight—until their primary team gets there. So, you know, if a patient is on a different team and 
they don't know much about the patient, other than just, you know, certain, certain things their primary-like their chief complaints and what's going on primarily with 'em ,you know, they'll let the primary team in the morning make decisions to progress their care, or to change their care, or this, and that. Which, when you think about it, like, if you're in an SICU, you know, time is a very valuable thing so, if you don't, like act on certain situations, or you wait, it's just kind of delaying care for the patient, until the next day when the primary team comes in. (\#21, p. 2) This quote demonstrates that the power differential is often manipulated subtly or perhaps unknowingly by physicians. In these previous examples, the physicians covering for other physicians and working nightshift for the primary team would not take full responsibility in learning everything they could about the patients they were responsible for, which impacted the physicians' ability to provide orders, thus hampering the ability of the SICU nurse to function effectively.

\section{2.b. Patient Advocacy}

Nurse participants recognized both the need to be patient advocates and the necessity of advocating for their patients with families and physicians. Patient advocacy was present when the nurse advocated for the patient's safety and care through critical thinking, assertiveness, and a diligent response. For example, in a forthcoming example, a nurse went above a resident and paged the attending after repeated attempts were made to notify the resident of a change in the patient's condition. Thus, patient advocacy was identified as a theme because the nurse participants identified multiples examples where needs were acted upon in order to improve the patient's condition and/or care. The following nurse advocated for her patient by standing up to a physician in front of nurses 
during change-of-shift. She advocated for the patient and did not back down from a physician attempting to leverage his power against hers, as follows:

I had admitted an MVA [motor vehicle accident]. I called KODA immediately and they said that they would be up around shift change even though this was in the middle of the night. I talked to the husband, discussed code status. He said that she would not want to be coded she would not want to be in a nursing home on life support. So, he made her a D.N.R. [do-not-resuscitate]. And I keep calling KODA, before KODA can get up there, she starts to code. And I said the patient is a DNR and the order is signed and in the computer. She was I think PEA [pulseless electrical activity] for maybe 10 minutes while the KODA liaison was in the room with the husband. And she comes out and says, he would like to donate if possible, so after she's been in PEA for about 10 minutes—we start coding her. There is family everywhere. And the patient is in PEA and he's ordering, atropine, and I see someone starting a Neo [phenylephrine] drip and I'm like, 'that's PEA,' he says, 'no, she has a pulse.' I said, 'no, I'm looking at it, I'm looking at it from the doorway, and I can see, I know that rhythm has no pulse.' And so, he yells at me that, 'there is a pulse!' And I say, 'I don't believe that and atropine won't work for PEA.' And I said, 'her family did not want her to be coded.' And in front of every family and everyone he tells me to back-off the patient, he's the physician. And I said, 'no, I'm her nurse. And I'm not going anywhere [laughs].' (\#19, p. 5-6)

This quote captures the nurse's (19) ability to advocate for the patient despite being in front of an audience of her nurse peers and physicians. More importantly, this nurse 
participant stood up for the patient despite being under the scrutiny of a physician who had a conflict-of-interest with KODA. Interestingly, this nurse participant described the attending physician's demeanor after the incident: "Yeah. Yeah. And so [attending physician] and I don't talk. He apologized to me afterwards, but of course it wasn't in public" (\#19, p. 6).

In another example of patient advocacy, a nurse (17) on the same unit stood up for a patient who did not want to have a feeding tube placed by having a discussion with the attending physician. The nurse participant goes on to say that she was successful in her advocacy, because the attending physician conceded and let the patient guide his own care:

But, like, I really feel like, while I have compromised in some ways-I will stop at absolutely nothing to tell you what I feel is right by that person and it may not even be what I agree with—it may not be what I personally agree with, but if that's what they want, then by God, I will be the voice that elects to tell Dr. [attending physician] that, like, I don't care, and I will not stop, like, we had a patient the other day who was like, 'I'm okay with getting a trach, because that can be reversed,' and he's like, 'I know a PEG can be reversed, but I, like, love food, and I'm not being fed through my belly.' And so, like, they were like, 'this is ridiculous, why would we trach somebody?' And he's like, then I won't eat, you can give me TPN until I can pass a swallow study. And he was intelligent. He was an old cardiologist and he was like, 'I do not want a f------ feeding tube.' And [attending physician] was, like trying to convince him and I pulled [attending physician] out of the room and I'm like, 'can I talk to you for a minute? This- 
you're way out of line.' I was telling him just like I would tell a friend, if they were like, trying to convince my friend to do something I didn't feel like they should do. I just told him, 'dude, you're out of line.' He said, 'why do you think that?' And I explained, like, 'he clearly doesn't want it. He's had this will written for 20 years, he doesn't want it and he's obviously lucid enough right now to tell you he doesn't f------ want it.' He said, 'so.' I said, 'why do you want him to have it so bad?' He's like, 'because he can't go to long-term care without food,' 'so, you want him to go to long-term care? You just want him out of the ICU?' He's like, 'well,' and I'm like, 'yeah, so say yes, because you're saying yes,' and he's like, 'yes,' and he's like 'I, I do want him to go to long-term care.' I was like, 'but he doesn't want that. He wants to go to rehab.' And I was like, 'he can move, he can go to rehab, like, yeah, it's going to take him longer, but like, he obviously has the dollars, he's got insurance. Send him to rehab and let him have TPN. If that's what makes him feel dignified let him do it, what's it matter?' And he's like, ‘well, you know, this, you know, it's, you know...' He's like looking for an answer and I said, 'why do you feel like he needs a PEG tube, so bad, you know? Like what, what is it?' And he couldn't give me an answer, there's no medical necessity and so he's like, 'it's just that he needs, you know, that's what he needed to get out of the unit.' To get off their list and I'm like, 'but that's not what he wants' and he's like, 'okay'. (\#17, p. 30-31)

Unlike the previous example where the nurse participant had to advocate for the patient in front of an audience, this nurse participant (17) was able to calmly pull the attending physician aside and reiterate the patient's treatment preferences so that they were adhered 
to and not ignored. Thus, this nurse was a patient advocate by calmly communicating the patient's treatment preferences to the attending physician during their rounding time.

Thus, this nurse was a partner in the patient's care that advocated positively and effectively.

Another nurse (2) was disturbed by other nurses not caring for their patients; thus to advocate for the patients and ensure they were getting the care they deserved, this particular nurse participant advocated for her patients the following way:

Because they don't wash their hair. It's the number of times that I've gone to do oral care and I've made a mark on the oral care package and can definitely, definitively tell you that no oral care has been done since I last did it and sometimes it's been days since I've had that patient. It's the sometimes we mark the chucks under the patient to see if they're getting turned or moved. I want to know that our patients are being moved. If I come back to work and my patient's in the exact same position, you're getting a PSN [Patient Safety Network - form of digital occurrence reporting]. I'm really sick of patients getting skin breakdown, getting CAUTI's, getting CLABSI's, getting just everything because we're, you know, what I don't want to pull out their Foley because it's just more convenient. That's bulls---. Pull your Foley out. Oh but the condom caths don't stay on. Then put'em in a diaper. Or, oh my gosh, clean your patient up. Oh no anything but that. I don't wanna give'em their stool softener because then they're gonna s---. That's their body's job. Their body lives to s---. If my patient's not s------, I'm not doing my job. Because I'm not taking care of their body. (\#2, p. 28) 
In the following example, the nurse was being a patient advocate, because she was not actually caring for the particular patient - another nurse was. Thus, this nurse participant was advocating for the other nurse's patient, because she felt that she was not providing adequate care. When asked about a situation where she knew the right thing to do for the patient but could not act, she had this to say about another nurse (13) working that particular night:

I had a patient and blood pressures had been really low, kept telling the nurse that they were really low and nothing was done about it. No, by the nurse. Yeah. Nothing was being done about it, constant reminders, hey, your blood pressure's this over this and then finally the patient ended up almost coding. So I feel like there, he was not getting adequate care at all. I think they did, but by then we were close to coding. I was just there. I was one of the co-workers. The patient just wasn't getting the care. If we had jumped on that blood pressure sooner, we wouldn't have been close to coding the patient. They would have had a better outcome. (\#13, p. 1- 2)

Many nurse participants described instances of patient advocacy. For example, nurse participants described patient advocacy as a way to connect with the family and enlist their support in the care of the patient. For example, by helping with turning the patient, the family was an active participant in the patient's care. One nurse participant (5) - who is a new nurse described going “over a resident's head" by calling the attending physician because the resident was ignoring her reported assessment that the patient's flap had lost blood flow and needed to be rushed to the operating room describing the incident as follows: 
I had a patient that, um, was plastic surgery. He had a free-flap and he had been in and out of the hospital, you know, for years and years - they brought him up to the SICU. I had him for a couple days, but my last night that I had him that week, um, I had to call the plastic surgeon in, like the attending plastic surgeon in at midnight because his flap had literally fallen off his face, it was like sitting on his shoulder. And I had called the resident, and the resident was like, 'oh, is it, is it really much lower than it was before like dayshift had left? I was just up there at like 6:30 pm myself.' And I said, 'well, I'm telling you it doesn't look what it looks like when I first came on my shift and assessed it with the dayshift nurse.' So, I was like, 'I would really prefer, you know, you come up here and look at it.' And then the next thing I know, I'm like, 'I'm going to call the attending and I called the attending in.' I explained everything. I said, 'you know, he still has a pulse in his flap, but you know it was pretty much hanging off of his face onto his shoulders.' He gave me all these orders over the phone to have everything ready and that he would be there in less than 20 minutes. And 20 minutes later he comes up, like we pretty much perform a mini-surgery at bedside and so that they could take him back to the O.R. the next morning and he pretty much thanked me up and down for calling him, because I was, you know, basically saved this guy's life. (\#5, p. 11-12)

\section{2.c. Positive Nursing - "Doing the Right Thing"}

Positive nursing not only describes the nurses' attitudes about their nursing perspectives, but also how they pitch-in and work together as a team. Teamwork is what facilitates this positive nursing phenomenon. Furthermore, "doing the right thing" is 
couched within this context of optimistic, positive nursing. For example, a nurse participant (10) in one of the SICU's was able to capture this sentiment, particularly as she described what is helpful about the SICU teamwork:

I don't mind doing tasks and helping people. I think the whole point of having a team in a group aspect like that is that and what separates SICU from like the floors is that we help each other. (\#10, p. 29)

Interestingly, a nurse (11) described nursing in a positive way and how even though it is difficult in the SICU, it is the satisfaction of "doing the right thing" that allows this nurse to keep coming back:

Yeah. So this is what in and this is something I've kind of been thinking about the last few years about like and I was telling [my wife] about this I was like this is like we were talking about work and it was like as a fun or enjoyment scale it's probably a zero to two. But a satisfaction out of zero, it's a 10 it's a 12 . So if you go at it as being satisfied not having fun or whatever. And, and the fun will come out of being satisfied or the, the perspective of being kind of feeling whole. As being satisfied not being that stupid idea of find something you love every day or find a job you love doing every day and you'll never work again. That's just dumb. (\#11, p. 30-31)

In the context of "doing the right thing", one nurse participant (10) stated that she would have to resign if she felt that she was unable to do the right thing for her patients. Thus, the following quote exemplifies her commitment to doing the right thing for her patients in the SICU: 
That bugs me, in the sense of it makes me reevaluate like, okay, who would I give that nurse as a patient next time? Because sometimes you just have to give people time to grow and learn from their mistakes. And it's unfortunate because we do have people's lives at hand. You know, one mistake by me could mean someone's death essentially and by the same as a physician, it could be that same way, so I think that if it was like a severe situation that was constantly happening, I probably would have to put my resignation in, but I don't know if I would want to put my resignation in because I don't know if anybody else would follow-up with that, if that makes sense. I feel like it would be better to like reeducate people and promote training and like what to do in certain situations, rather than just like resigning and backing away. The, I don't feel like on our unit - sorry I'm going through all possible things that would make me resign. (\#10, p. 34)

Another nurse participant (12) said she coped with the work in the SICU by talking with the nurses she worked with that night in the charting area post-shift. She said it was a form of decompression and helped her do the right thing to process the experiences she had in the SICU:

And I think that I recognize that as like a way of, um, like decompressing and, like, I mean therapy [laughs] and I'm a huge advocate, I mean, I'm into therapy, like I 100\%-it's needed. And they experience it with you and, and they know even if they weren't in the situation with you, they understand what happens and our reactions to it and what we have to do, what we don't have to do, and whatever it is, you know, what I mean —it's just easier to talk to somebody who's 
in the healthcare setting and particularly the same unit as you, and even better the same room as you when it happens. (\#12, p. 7)

Nurse participants discussed numerous positive aspects about nursing, about the job in SICU, and about the service aspects of the job that made it worthwhile. Thus, not only was "doing your best" for the patient a major theme throughout the nurse participant's responses, but it was also interconnected with positivity about nursing, because so many of the participants felt that they were doing the right thing if they were able to do their best for the patient. Thus, it takes a dedicated nurse and patient advocate to remain in the SICU environment, which according to the nurse participants is often short-staffed, lacking in resources, and occasionally suffers from breakdowns in communication, yet the nurses stay and care for the patients. For example, one participant (3) who has worked in the SICU for many years said the following about her decision to still remain at the bedside:

Why do I put this undue stress on myself? And the other thought is, you know [laughs] why it's kind of conflicting because then I think, well who wouldn't want to do this? Who wouldn't want to take care of the sickest of the sick? Um. Isn't that you know, isn't that what nursing is? I mean. When I graduated, I knew that that was what I wanted to do. And I think how fortunate am I to still have that experience. (\#3, p. 14)

Interestingly, another nurse (1) - who was found to have a great emotional awareness about her job said the following about why she loves to work in the SICU: "That's how I describe the unbearable experiences. We do a really great job of finding the ability to create joy in a s----- environment" $(2$, p. 35$)$. Nurse participants discussed treating SICU 
patients like family members. Nurse participants loved the job for the value in the human spirit. Nurse participants said it was rewarding to see former patients walk in rehab. One experienced nurse who devoted her life to the SICU said the following about young nurses:

I do like SICU. I do like the pace. I do like that type of patient. I like that we have a variety. I like that it's a teaching hospital. I like that we have a lot of young nurses. And you know they're interested in doing a good job. And there's a lot of jobs you can go work at and not work as hard as you work in the SICU. So I think it draws a quality person. (\#1, p. 9)

Interestingly, nurse participants advocated for their patients in public and in private, and in the midst of confrontation, and also collegial communication. Thus, it is evident that patient advocacy was not a problem for seasoned nurses. Moreover, not only did the nurse participants advocate for their patients through actions, but also through their decisions and choices for remaining in the SICU environment - because they enjoy the work due to the difficulty level and the ability to serve fellow humans in a vulnerable time of need while "doing the right thing". Nurse participants knew what their patients needed, but they were unable to provide it in the case of physician-nurse power differentials. For example, one nurse participant deferred to the physician simply because they were the ones writing orders and were therefore viewed as authority figure. Yet, this nurse participant was morally distressed about patient care because she knew the right action to take, but was impeded by physicians. Multiple nurse participants described the same frustration with physicians using their status and knowledge as leverage in interactions with families, patients, and other nurses. However, the nurse participants 
recognized this use of power because it deterred focus on the patient's well-being and outcome, thus it was not "doing the right thing". Nurse participants were patient advocates and maintained a positive attitude and professional approach towards nursing in an effort to focus on "doing the right" thing in patient care situations. Regrettably, moral distress occurred when the nurse participants were not able to advocate for their patients and "do the right thing" through either their action or inaction in a particular patient care situations.

\section{Theme 3 - Surviving Moral Distress in the SICU Environment-Magnifying Stress \& Self-Preservation}

The third identified theme highlighted the second part of the process of moral distress in which the SICU environment magnifies stress, thus the nurse participants had to resort to self-perseveration in order to survive. For example, the sub-themes highlighted within the theme of surviving the onslaught of an unethical environment are the result of moral distress, some lack of staffing that affected care poorly due to the participant not being able to provide what they deem the best care possible. Within each interview, the nurse participants described a root cause of moral distress. The moral distress experienced by most of the nurse participants was a result of their concerns in the context of end-of-life, such as the suffering of their patient, as well as questioning whether or not the treatments, given the patient's prognosis, were necessary. In many instances, the root cause of moral distress was the result of a combination of poor staffing, incompetent RNs combined with lack of SICU experience, lack of resources, and lack of support from management, management problems, demands of additional "unnecessary" chart audits, and the additional responsibilities which prevented nurse 
participants from being able to provide nursing care to the best of their ability.

Furthermore, there were both positive and negative communication issues prevalent throughout the interviews. Not only did ineffective communication between a new resident physician and an experienced nightshift nurse lead to morally distressing patient care situations, but effective communication allowed for morally distressed nurses to cope with and be supportive of each other so that they could focus on caring for their patients throughout their shift in the SICU.

Surviving moral distress in the SICU environment is a theme that conveys the strong-will that all the nurse participants demonstrated in the face of moral distress in the SICU. Moreover, this theme summarizes the majority of morally distressing issues for the nurse participants, signifying a testament to the ability of nurse participants to cope with moral distress. Poor patient care was distressing to the SICU nurses because they characterized this as "unethical" and were powerless to change the patient outcome during the moral distress. The third theme highlighted the second part of the process of moral distress, describing how the SICU environment magnifies the nurses' stress. The SICU environment magnifies stress because nurse participants described experiencing moral distress and being unable to stop the process of moral distress from unfolding. As a result, this led to their perceptions of "unethical" patient care. Thus, the nurse participants resorted to self-perseveration in order to survive "unethical" activities in the SICU environment.

Theme 3 is comprised of several sub-themes resulting from moral distress in the SICU, including: (a) nursing management problems, short-staffing, and lack of resources; (b) incompetent nurses lacking in SICU experience; and (c) demands of additional chart 
auditing and additional responsibilities. For example, lack of resources that negatively affected patient care due to the nurse participants not being able to provide what they deemed the best care possible. Within each sub-theme, a root cause of moral distress was identified. The moral distress experienced by most of the nurse participants was the result of concerns in the context of end-of-life, such as inquiries into patient suffering, as well as considering the treatments and prognosis involved and questioning whether or not the suffering was necessary.

\section{3.a. Nursing Management Problems, Short Staffing, and Lack of Resources}

Nurse participants noted that nursing managers were often rendered incompetent with poor staffing decisions and lack of resources, which contributed to their moral distress in the SICU environment. Thus, moral distress was characterized by problems with nursing management, poor staffing, and a general lack of resources for providing care in the SICU. Interestingly, one nurse (2) described the problems in the SICU related to not having PT and OT staff consistently available during each shift to mobilize patients so that they will have better outcomes and a shorter length of stay, as follows:

Like do your f------ job. There's no reason why my 10-patients on my 10-bed unit can't all be mobilized every day. Or even make PT just assess them every day. I don't know. It's just frustrating. The consistently most intense bothersome thing is all the preventative preventable things that we, that our patients go through. The skin breakdown that didn't need to happen because our patients aren't getting turned or we're only turning their torso and not their head so they get a deep tissue pressure injury on the back of their scalp. Most of the time people don't even notice it. (\#2, p. 28) 
This same nurse participant (2) relayed that there were multiple levels of incompetence in management as well as other staff, because she found that 8 of 10 patients had skin breakdown whether from pressure resulting from being in one place too long or being in soiled linens:

I complained to [Nurse Manger] maybe in December because just walking around I did a headcount my personal self and 8 out of our 10 patients had skin breakdown. Eight. The two patients that didn't have skin breakdown were ambulatory. So I guarantee that for that particular week if they had not been ambulatory we would've had 10 out of 10 patients with skin breakdown—of some kind. Like we had a deep-tissue pressure injury uh brewing on the back of a head. We had urine scald because people don't check diapers. Or they'll just pad it and be like oh it's fine. But it's all gone to the back. And they just leave it. I just I don't understand it. Like — take care of your patients. I don't... I don't get it. (\#2, p. 29)

Nurse participants argued that many of their concerns were from lack of staffing in SICU, noting that this was due to incompetent nursing management, as well as budget decisions. Therefore, these issues are interconnected. For example, one charge nurse had consulted the chief nursing officer and the chief medical director of the hospital organization over the short staffing issue problem impacting patient care. Fortunately, this nurse (17) was "heard" in her communication, but not by the chief nursing officer-by the medical director. Thus, this nurse felt that upper administrative management of nursing did not support bedside level staff nurses: 
So, you've got your chain-of-command, like you're supposed to follow, are they not communicating effectively in there? And, they'd rather-so they don't get in trouble — rather, say, 'everything's fine, everything's fine.' To make it so that they're not in trouble, because if their unit's in trouble that falls back on the manager, of course, so, are the people above you communicating effectively and not only communicating, but are they being honest about what they are communicating? Um. And I would say—no, because ever since I had that conversation with him [chief medical officer], things, he's been much more visible, he comes in, touches base with me regularly, and is like, 'how are things going, like do you feel like you've had issues?' (\#17, p. 17)

Nurse participants also noted that they do not have the necessary ancillary staff and other healthcare professionals (i.e., physical therapy and occupational therapy) to mobilize patients in the SICU, which is required in their acute and traumatic states. For example, nurse participants revealed that physical therapy and occupational therapy are only scheduled to work six days a week during the dayshift. However, nurse participants correctly noted that if they are sick enough to need therapy six days a week, then they need it every day. Additionally, one nurse participant (6) - described never having enough nursing staff to mobilize all of their patients every day in SICU, as follows:

Well, half of these patients, we trach too many people-half of these patients I feel like if we could like really focus on doing like spontaneous breathing trials every day taking 'em off their sedation and stuff like that, then we wouldn't have to trach them, but you can't really do that because you've got to kind of keep them calm because your other patient is going crazy and so like I feel like if our staffing 
was a little bit better and you had you know someone you had a charge nurse or something to watch your other patient you could be in there with that patient really trying to work on getting them off the vent instead of just kind of keeping them snowed for like 4 days and you're like, oh, I don't know why they're not waking up like they all, I don't know why they can't get off the vent, I guess we'll have to trach 'em and so, it, I just feel like there's so many people that just get trached and have an even longer hospital stay because we can't give them the care that they really truly deserve. (\#6, p. 11)

Moreover, an experienced nurse participant described staffing issues in the SICU and the inability to mobilize patients appropriately, as follows:

Just in general, I don't feel I have the resources to physically turn people as often as they should be turned or gotten out of bed, particularly the gotten out of bed, you know, I think that's why I rely on PT so much is people are big nowadays and to safely get somebody up really takes 2 or 3 people. They have a piece of equipment that that kinda looks like a birdcage it's a pair of pants that you like you can put people's legs through and then it's a big sling to it so they can't fall off if they sat down they sit in this sling type apparatus. But they don't, you know, they don't have anything like that in the between periods of short-staffing and they, it's just kind of drifted to where we don't get people up anymore. The first time - PT gets 'em up the first time. Very rarely see nurses really take the initiative to get people out of bed like they used to. It used to kind of more expected that you would get people out of bed at least once on your shift unless they were attached to the ventilator and, of course, there's a lot of hospitals where 
that doesn't happen. They get people up on ventilators. I think that's true. And then it's really hard to get the quality back up there once staffing improves. It's hard to lead people from this is how we do it. Now we don't get people up. It's hard to move people back to that. Once you get better staffing. Then automatically just bring 'em back. (\#1, p. 5-6)

Furthermore, due to situations of short-staffing on nightshift, nurse participants noted that they felt unprepared to provide adequate care, because they did not have enough staff, supplies, or resources. The SICUs are short-staffed poorly at night due to lack of staff with experience: "Well, obviously we're not clinically giving the ideal patient care, because we don't have the staff, we don't have the supplies, we don't have... what we need. We have the bare necessities to prevent infections" (\#2, p. 38). Interestingly, another nurse participant cited prior experience in nursing management explaining that she had to scrutinize everything she did, and also everything everybody else does. This nurse participant relayed that people will lie to protect themselves and, thus, one has to be diligent as a nurse and be observant to discover how a situation of patient harm occurred regardless of one's relationship with the individual in the hospital organization:

Well I think the biggest one was when I was a director... and there was a baby who died. And so we did this preliminary investigation. I've always been around some interesting cases. So the baby ended up dying because of bad care. Bad care from nurses and bad care from a doctor. So, I learned from that that there was a lot of people who would tell a lot of lies to protect themselves and if you were ignorant you would fall victim to that. So you have to be pretty diligent about 
doing things right. You have to scrutinize everything you do and you have to scrutinize everything everybody else does. And you have to be the one who is the constant watchdog for everybody. So you have to watch yourself. You have to watch the pharmacy. You have to watch the physicians. You have to watch everybody do everything. It is exhausting. [laughs][laughs]. I don't know if you do that in your practice, but it exhausts me when I work. (\#4, p. 8-9) Nurse participants revealed the increased responsibilities and incompetence from nursing management makes nursing more difficult, such as peer chart audits and staffing decisions. The healthcare organization began a new initiative to improve charting by mandating two peer chart audits to be completed monthly by each SICU nurse. Numerous nurse participants bemoaned this new responsibility. Nurse participants argued that peer chart audits are management's responsibility because adults with professional licenses should be evaluated by administration/management—not their peers. Nurse participants said the additional responsibility and pressure to complete this new task was adding to the growing list of charting responsibilities and taking time away from patient care duties. Another nurse participant specifically noted that the unit manager wanted nursing staff to come into the hospital on their off time to complete chart audits and when asked how this is saving money for the budget, the manager asked the nurse to go home, but the nurse refused. This nurse participant discussed her experience with a previous director whom she viewed as incompetent. The nurse participant claimed that this particular nursing director was incompetent, vicious, and would make staff feel humiliated and that she gained a certain amount of power from standing up to the nurse manager and not leaving work: 
I mean, we've had incompetent management [laughs]. Those were definitely powerless years, [laughs]. And some of it was-too stupid to talk to, and didn't care—she really only cared about her higher-up's talk. Well, just not walking out the door was power, you know? The fact that she didn't run me off... I felt I did get a certain amount of power, because that was definitely her [nurse director] goal. And [another nurse] made points that she was very degrading to me in front of other people, and she humiliated me, but I never personally felt humiliated, because I had no respect to her to start with. If it had come from a source that I valued, maybe I would've taken it seriously. But, she—-to me—was always just someone who never should have been in that position. (\#1, p. 7-8)

Nurse participants felt that other healthcare team members, such as the unit case manager, were judgmental about patients who abuse drugs and are admitted to the SICU from traumatic accidents. For example, one nurse participant (2) noted that the case manager judged a patient who used drugs while the nurse participant was attempting to advocate for the patient and "do the right thing", but the case manager was not being helpful due to the patient's history of drug abuse:

I feel like she takes one look at a patient and will do everything, or nothing for them, and if you mention drugs - she'll do nothing for them. I had a patient that was, I don't know, she was either in her late teens or early 20 s, and she, um, had vegetation on her mitral valve - her parents had shot her up for the first time when she was 13 , um, I'm pretty sure they had sold her a couple times, in her life, um, this is not a kid that had had any support, but because she was a drug user, and she felt like she couldn't kick it, like she was having a hard time, literally the hospital, 
um, said that she needed to get a heart valve transplant, but because she had been in and out of the hospital so much recently they said that they, um, she had to remain in the hospital until it was transplant time, because they didn't trust her ,or whatever. Um, she had an eighth grade education, and she expressed to me that she really just wanted to get her GED, and I was like, 'you know what, that's f----- rad. Good for you. You're sitting here, you're not doing anything, you gotta be in the hospital, let's see if we can get you some resources. Or even help you get into a halfway house.' Because she told me that she wanted to break-up with her boyfriend, because he used, and, um, he wasn't supportive and her mom had gotten clean and her mom really wanted the boyfriend out of the picture, but like her mom couldn't help her, because her mom was living in a sketchy environment. Um. You see a lot with addicts where they just like, have these big dreams, but they just don't know how to make it happen, like a lot of addicts want to get clean they don't end up being able to emotionally do the work that goes into it, but if you support 'em you can get 'em a better shot. I told [case manager] that this little girl wanted to get her GED and that she was interested when she was done with her recovery process, um, going to like a halfway house, or whatever, um, and [case manager] was like, 'well, we don't have any resources for that, that's not a thing we do.' And I was like, 'I'm pretty sure that we can help with that. They literally brought an alligator. Like a baby alligator to the burn unit for a patient who just said he wanted to wrestle an alligator before he died. If we can bring an alligator from somewhere I'm pretty sure we can get a packet of 
information so a girl can get her f------ GED.' Pretty sure we can probably Google that like... but let's not do our job too thoroughly [case manager]. (\#2, p. 22)

Problems with short-staffing were exemplified by one nurse participant (6) who said she was "burned out" and has subsequently left the SICU:

I feel like a lot of our issues come from staffing. Because I have nothing to do with staffing - like if I come in and we have 6 nurses and so we actually have a charge nurse out of staffing but 5 of us has 4 [patients], someone's getting pulled - it doesn't matter. Like we finally got to the point where our unit was staffed, but the rest of the hospital wasn't, so it was pointless basically like because we'd get pulled to other units. (\#6, p. 11)

\section{3.b. Incompetent RNs Lacking in SICU Experience}

Incompetent nurses and the lack of SICU experience were mentioned frequently by nurse participants as sources of their moral distress. One nurse participant (7) who began their nursing career in the SICU said the following:

Powerless. Yeah, I mean there's nothing like super outstanding, that one case I was talking about was really frustrating for me. But I feel like a lot of my frustration. Comes from like a self-critical standpoint like I'm frustrated with like my inability to, you know, maybe have done something better. But that's not really frustration with like if, if and, if I ever feel like powerless, it's usually powerless because I don't know what I'm supposed to be doing that's like one of the feelings particularly on orientation that I really despise. Um. Is just like feeling like you're just standing there with like not a clue in the world as to what 
you're supposed to be doing. I think that's one of the more frustrating things I've felt at work. (\#7, p. 28)

Interestingly, one SICU nurse (17) noted that the incompetence of nurses is a result of the training they receive as they are going through onboarding and acclimation to the unit. She described the preparation process and how new graduates and new nurses in the SICU are evaluated:

If somebody said, 'hey these are things you can do to troubleshoot,' but, you don't learn that in your training, really, like when you get oriented, you get trained how to-like function and manage your time, because that's ultimately all the management really cares about, can you not kill the patient, can you give them good care, can you not kill them, and can you give them their assessments, in which they're legally ordered, and can you document that legally. Like, to methe big picture - is those are the things that are looked at, they're not looked at the actual like - if you think about what the word care means, like, the actual care that they get - do they really give care? Or are you just doing things to them, and to me like that's the most like, like that's the worst part about being a bedside nurse, it's like are you caring for them or are you f------ them up? Like... [laughs]. (\#17, p. 10)

This same nurse (17) - noted the difficulty of working short staffed and with nurses who are not experienced, because it impacted her care and ability to communicate with family members who were concerned about their loved one:

Yeah, it blows, but somebody's got to do it man, you know, it's like when you can't even do that, like I just feel like a bad human more than a bad guy, I mean, 
like, I feel, like a bad nurse, but you just feel, like, I mean, I feel like a deep sense of guilt, because I feel like not only am I not giving this person respect, but I'm also disrespecting their family by not being able to keep them appropriately informed throughout the course of my day and you can't, you can't- the truth is that there is no fixable thing that I think, except more staffing and more competent staff members, you know? (\#17, p. 16)

Interestingly, another nurse (8) described the experience working with incompetent nurses:

Kind of like, nervous. When you work with a person that's incompetent, you're kind of like nervous for that patient, I mean it doesn't do anything to me, like, I'm not you know legally responsible for the care that they're providing. And it could be their best, but I think a certain, you can fix incompetence. You know, if someone's lazy, but that's a character flaw on them, you know, like maybe they don't get up and turn their person like they should, but if you're like unintelligent, I think that's inexcusable. Because you can fix intelligence. And you can fix laziness, but I think it's responsibility in the medical profession to like, like you gotta be as educated and up-to-date as we can be, because someone's life depends on it. So, I'd rather have an intelligent lazy nurse taking care of me, than an overachieving, ignorant nurse. 'Cause, when I think that, s--- hits the fan, you can clear off the lazy. You know, but if it's I'm circling the drain, I'd rather have that person who can dust off the lazy and use their brain versus the person that's running around with no clue what's going on. (\#8, p. 9) 
Furthermore, another nurse (20) noted that often women might be disrespectful to each other, which is immature and not the model of how an experienced SICU nurse ought to act in caring for sick, vulnerable patients. She went on to discuss that it made work more difficult:

That's just one thing that like you can hate somebody, but you still need to be able to be professional, and your common goal is the patient and that needs to be taken care of and if you're just, if you can't be a part of that, then you don't need to be taking care of anybody, you need to find yourself a little desk job for a little hermit, but I don't know. I just, that's really the only thing that comes to mind, is that someone who would blatantly just not help you just because they don't like you, and like this cattiness sometimes. Because, it's like, predominately, you know, a girl profession, you have, we work with more girls, we do actually have a lot of guys, and a lot of other places I've worked at, it's been like $90 \%$ girls that you work with, and you see that a lot like, oh, I don't like her so I'm just going to sit over here and pretend I don't hear her pump going off and the pressor evidently bottoms out, and, you know, it becomes an issue when it should have never been an issue. (\#20, p. 17)

\section{3.c. Demands of Additional Charting and Responsibilities}

The demands of additional charting and responsibilities, including scales, assessments, and evaluations that occur hourly, encompasses multiple tasks that SICU nurses are responsible for completing in a 12-hour shift. In particular, one nurse (19) noted the following about the lack of support from management regarding all of these extra demands and responsibilities: 
There's just no backing from management for anything, you know? Management wants you to do all these extra things, which are things that, yeah, you know, like early mobility, yes, we should be doing that. Um. But, then the chart audits and the HAI [hospital-associated infection] rounding forms and things and it's like all of these things are taking away from what I joined the field for, and yet if I'm trying to advocate for... I mean, I understand the importance of it, as far as, you know, research, so that we can, you know, because they call me the CAUTI-Nazi on the floor I'm always wanting to pull 'em. But. I'm also trying to take care of my patients, and so, I feel I'm trying to take care of my two [patients], while keeping an eye on the other eight, and the four other nurses and then I'm supposed to fill-out these forms, but somehow take care of my two patients and then keep an eye on eight other patients, and then four other nurses, and then they can't stick, so I go in and try to get another IV and [a step-down unit] is calling me for an IV, again. Um. Yeah. It's...You don't have enough time to do stuff for the patient, because you're being pulled in a lot of different directions. And then, when you voice that there's no backing from management. No. A charge nurse should never be taking patients unless it's like worst-case scenario. There needs to be someone who's kinda-sort-of a help-all resource. (\#19, p. 10)

One nurse recounted her going to the chief medical director about the dire short staffing problem. She did not view this as tattling, but rather she went "over-the-head" of the chief nursing officer and consulted the chief medical officer, because she was not receiving the proper support from the chief nursing officer. Thus, this nurse (17) took the additional responsibility of reporting staffing needs to leadership and administration, 
because it needed to be done and because SICU nurses were having to take three patients and the norm is usually two patients. This nurse described going above and beyond her duties to advocate for the entire unit:

I try to like, go to the people that I think can make change, and that's not to like, be a tattle, but it's just like, to get things done, like I really believe in like, that being quiet is not the answer, you know, people say, 'oh, I don't wanna be a tattletail,' well, I don't want to be the person who complains about staffing. Well, I don't either. So, then we have to do something to fix it, like I'm not a complainer by nature, I'm a problem-solver, and so like, I see that there's a problem, and I feel that it needs to be fixed, and I think that it's truly a disservice to, you know, nurses and anyone in healthcare, to be placed constantly in a position where you're set-up to fail, and I just think that's bad, like that's and, if anything it's bad karma, like I feel like, it's bad to like put someone in that position, which goes back to why I didn't give the new grad the three patients, but they probably would have probably gotten even more garbage care than what I gave'em, because she just can't, you know, you can't manage your time, you're learning or you're trying to like, you know check your pupils, you know, for 20 minutes like that's part of your assessment like, you're just, you can't see, or you know the forest for the trees, like, but I don't know. [laughs]. (\#17, p. 18-19)

Nurse participants noted the demands of additional charting and how evaluating and auditing peers' charting and documentation should be a task of management - not other bedside nurses. Documentation when caring for two critically ill patients is tedious and complex. However, nurse participants noted that the process of charting all information in 
a shift accurately and punctually is made more difficult by the new peer chart auditing expectation. Interestingly, with all the mandatory documentation standards, a majority of the nurses did not approve of this policy of additional peer chart auditing. One nurse participant (6) who recently left her job in the SICU described the additional demands of peer chart auditing:

This is more about like why. The additional stuff like the additional roles we have to take on as nurses, like you can't just like go to work, take care of patients, and leave like, they want you to just like start getting more certifications and start doing like all this additional stuff, but like recently we, we had to like she introduced, um, a chart audit, so twice a month every single month we have to do two audits, on our peers, on their charting, which I feel like, that's ridiculous because we're all adults - we all have licenses. Like I should not have to look at someone else's charting, and make sure they're doing their job. (\#6, p. 4-5) In contrast, another nurse participant (10), unlike other bedside nurses interviewed, reported that from a charge nurse perspective the peer chart audits were necessary to keep nurses accountable for their documentation because it impacted patient care:

The way we do certain things, like with our line documentation and stuff, if lines weren't documented correctly, or if a count wasn't done, or if the crash cart wasn't done, just the little menial tasks that again, just build up to be a lot more like detailed-oriented and stuff, I wouldn't say that ever, like, taking care of patients is ever the reason that I would want to quit my job. (\#10, p. 13) Specifically, regarding peer chart audits and documentation pertaining to lines and dates, this particular nurse (10) felt that the process of auditing and documentation facilitated 
accountability. This nurse was a fan of peer chart audits and felt that accurate documentation and holding one's peers accountable to such high standards is part of one's job description to make sure charting standards are carried out. Thus, she had the following to say about accountability and following-up on nurses to make sure they are doing what they are supposed to in their documentation and care:

And for you to say like, 'oh yeah, well I cleaned this, I did that.' But did you really? Like the patient's still lying in the same position that they were in when I left. Did you really do it? Or are you just saying you did it, because it was one more thing to check-off your box? I think people have to take their own responsibility to hold themselves accountable for that stuff and I think putting a checklist and making a box of stuff doesn't do any good, because if anything else, it just makes people less likely to follow through with it. I'm also a rule-follower, so yes, I'm going to do it no matter what. But if you start telling me like you have to check this box. (\#10, p. 16)

However, the majority of nurse participants interviewed felt that the peer chart audits were excessive and should not be the staff nurses' responsibility. Moreover, nurse participants noted that peer chart audits interfered with patient care, because evaluating peer's charting several times a month negatively impacted time for patient care with a task that they voiced was management's responsibility. Thus, the nurse participants were morally distressed because they felt that management was forcing bedside nurses to do management's work, which also prevented nurses from taking care of SICU patients in the process through this mandatory peer chart auditing. Furthermore, each nurse was required to complete two documentation audits for two different nurses' complete 
assessments. Provided all the other roles and responsibilities they had to complete in the SICU in a 12-hour shift, SICU nurses voiced that the peer chart audits took time away from patient care due to double-checking their co-workers' documentation. Alternatively, one nurse (10) felt that peer chart audits were a necessary duty, because the tasks ensured accountability to documentation standards, which ultimately promote quality patient care. This nurse believed that two chart reviews were excessive, but the task is necessary in order to promote consistency in care, such as verification that a bath or turn was performed:

The peer chart review audits, I don't mind. I think two a month is a little bit excessive. I feel like one would be sufficient. But, I think that's good, because, I feel, I think in nursing school we learn, you know, if you didn't chart it, you didn't do it. And when it comes down to a court of law, or legal situation, I'm very like, legal-minded person in a lot of aspects. If something were to happen five years from now, and they were to pull a case from, you know, way back when, if I didn't chart it, then it looks like I didn't do it. And am I going to remember giving that patient a bath? Or that I did whatever? However, I did it. (\#10, p. 15-16)

Thus, this nurse (10) felt that through consistent documentation she would be protected in a case of litigation. She went on to say that it is important to document accurately in order to keep track of what is being done to the patient; however, she also noted that it is not appropriate to expect nurses to verify a previous shift's nurse's tasks when they were not present: 
I think the other thing with the chart reviews is you know everyone has a slightly different style of charting. Totally fine. But, because there are standards, unfortunately set, it's part of your job description to make sure that those standards are carried out. I think it's - to me it's less to pinpoint people who are bad charters as it is a better way to educate you on, and remind you of what you're supposed to chart and do. So those, I don't have a problem with, so much, like I said maybe two a month is a little bit excessive maybe like you know 10 a year I think would be sufficient. Or something like that, that way you just kind of have a little break, if you just want to do seven in a, you know, if you have a really slow month and you just want to knock a bunch out—do it. Fine. So that portion I think is fine. The line documentation and verifying that other nurses did something I think is absolute bulls----. (\#10, p. 15-16)

Thus, this nurse saw the inherent value of accountability and accurate documentation. One way to facilitate this accountability was through peer chart audits. However, the consensus from the majority of nurse participants was that peer chart audits were extra work that management should be in-charge of, because bedside nurses were already responsible for enough charting. One nurse participant (12) who experienced a moral crescendo and no longer worked in the SICU actually refused to do peer chart audits, because the audits were being imposed with a threat of disciplinary action if they were not completed, as she described:

Our asses will get handed to us. Or you know there's so many chart audits b------and you know I think [charge nurse's name] like got called back to court several times because she's been a nurse for 30 years. And she would always say 
document, document, document - whether it's saying I know that they need this and it's not available, or, and what I did about it or just checking out the f-----thing. Yeah they got a bath. I haven't been in that room all f------ night, but they got a bath you know like it's that type of s---. I think I'm kind of naïve when it comes to that. I always, I'm a huge like karma, I always [laughs] feel like if I do the best that I can and I... I don't know I think I'm just naive that it probably won't happen and then I it I think it's unfortunate that we really don't know how to chart in order to protect ourselves if we were called to court. You know what I mean it could have been done and we just didn't check the box or whatever it is but I think I'm, I think I'm just hoping that if I stay like a decent person and a good nurse [laughs] maybe it just won't happen. [laughs]. (\#12, p. 15)

One nurse (20) had the following to say about peer chart audits:

Yeah, I mean, I would, if I was doing something wrong, I would be, like, just tell me what I'm doing wrong, don't make me do all this bulls--- to do, like to, you know, rat on other people, what they're doing wrong, I don't know, I feel, like, I think the audits are stupid, but I mean, if they want us to do them whatever. I don't really care about it. But, just do what you need to do and move on. I don't know [laughs]. (\#20, p. 22)

Nurse participants noted the perils of charting through an inability to provide quality patient care due to the emphasis on charting in general. For example, one experienced nurse had this to say about charting:

I think that patients would be a lot happier. And I think the nurses would feel like they give better care. And the life of their job better-if they felt like they gave 
better care, but nursing in general seems to emphasize charting. Like if you chart that it was done. It was done. So nurses seem to have a real fixation with charting. And I'm not a very good charter. I'll chart what I can, but my charting's usually kinda hurried and it's not very detailed. But, um, I feel like, I waste a lot time filling out scales that help you make a decision. Like, um, the Braden Scale. Well, if somebody's, you know, only been there 24 hours and they have a head injury, are we going to feed them that day? No. They might go to surgery. But, no. We're going to come back, and waste our time filling that form out every four hours, because you're supposed to fill that form out to document that we're doing everything to take care of that person, because we can't meet their nutritional needs at that point. And you know the restraint form is just a total time waster. They're doing this for patient safety. You know? Is the restraint too tight? Could they do without it, you know? But, you go through that list of questions every hour, on the hour. It just keeps us out of the patient's rooms - is really all it accomplishes. I wish there was another way where you could check a boxnothing's changed. You know? Um. I, I have been in that room you know, but, um, I don't know what the solution is to that problem. (\#1, p. 6-7)

This nurse (10) stated that she felt torn between the responsibilities of management in her charge nurse role and patient care:

"I think by the same token. The job description for me personally falls back a lot on picking up slack that upper management does not do. As well as picking up slack that staff does not do. So I feel like I'm kind of held responsible for both parties. And I think the unbearable side for me is multiple times like I will do 
$95 \%$ of the work and management or one other person might come in and do $3 \%$ of the work and they call it complete. And somebody else gets credit. That's my unbearable [laughs]." (\#10, p. 27-28)

Moreover, some nurse participants were placed in the position of a relief charge nurse on nightshift when there was not enough staff to have a designated charge nurse, and these nurse participants did not feel that it was a safe situation to care for patients due to lack of experience of some nurses in the SICU. For example, one nurse participant (5) did not feel "ready" to be thrown into a relief charge nurse role; however, due to shortstaffing she had to adapt:

Sometimes, I think, it's when I first started, or when they first tell me oh, '[name], you're going to be a relief charge nurse every so often,' I looked at them and I said, ‘you're freaking crazy! I'm not experienced for that. And they're like, 'yeah you are.' And I said, 'okay, well if something happens, I'm not going to be charge ever again.' [laughs]. Like, I don't, I mean, granted I mean you can make assignments I mean I feel like sometimes I feel guilty making assignments because I'm like, I hope I don't give somebody a s----- team where like you know they can't sit down and then I have maybe the two easiest patients on the unit and I'm just sitting there which I you know I try to be helpful to all my coworkers but like, sometimes, uh, me not wanting to go into work is like, for like personal reasons like, oh, you know I really wish that I could go to, um, my cousin's birthday party, but I can't because I've got to sleep pretty much the whole day to get ready to go back into work that night or you know we're going to be shortstaffed tonight so that's really going to suck. (\#5, p. 12) 
Thus, this particular nurse participant felt that she was placed in a position to act as a relief charge nurse too quickly, because she had the most experience on the unit. However, these nurse participants did not feel "ready" to assume the role and said they would not do so again, particularly not without the necessary training first. Some nurses were taking responsibility for their professional career development based on their experiences from the SICU, realizing that they did not want to be a bedside nurse forever due to the stress of moral distress associated with working in SICU.

\section{Theme 4 - The Stain of Moral Residue—Surviving \& Coping with the Impact on Integrity \& Values}

Theme 4 exemplifies how the experience of moral distress leaves its mark on the victim as moral residue. The nurse participants who experienced moral distress multiple times also had multiple experiences in the SICU that were frustrating. One nurse participant said she was "burned out". Many nurse participants said that they felt that they did not compromise their overall patient care, but named particular instances where their care was compromised and they could not do what they wanted to do to provide quality patient care, resulting in moral distress. Some of the sub-themes in this theme impacted the nurse participant's values and personal moral integrity. For example, many nurse participants noted a change in themselves or if they did not recognize these changes, those around them pointed them out to them. Furthermore, coping and survival is the main occurrence associated with the stain of moral residue. For example, there are positive or constructive ways of coping, as well as negative and more maladaptive ways of coping. However, when viewed respectfully and objectively, all coping is a response to 
some stressor; therefore, all coping is a response to the initial stressor - in this case, moral distress in the SICU environment.

Many of the nurse participants described positive coping behaviors; however, other nurses use more maladaptive and emotional coping behaviors in the context of the work that these nurse participants encountered on a weekly basis in the SICU.

Additionally, compartmentalization was noted as a coping mechanism used to reconcile the nurses' experiences of moral distress in the moment and perhaps to file away to process at a later date. Another sub-theme is that nurse participants noted that there are nurses who are "just punching the clock" and trying to "get through the day". These nurses do not try their best at their job and do not care as much, which nurse participants noticed and discussed.

This theme exemplifies how the experience of moral distress leaves its mark on the nurse victim. The nurse participants who experienced moral distress multiple times with multiple experiences in the SICU were frustrated. Theme 4 is comprised of several sub-themes, including: (a) surviving and coping with the impact on personal moral integrity and values; (b) compartmentalization; and (c) nurses "just punching clock". These sub-themes impact the nurse participant's values and personal moral integrity describing how they coped with the stress and survived. For example, many nurse participants who noted a change in themselves and those around them pointed out these changes to them. Furthermore, coping and survival was the predominant occurrence in the stain of moral residue. When considered in the context of the stressful work environment of the SICU, these nurse participants encountered multiple stressors on a daily basis. Thus, it was important to examine their coping behaviors and how they 
survived the stressful environment. Additionally, compartmentalization was noted as a coping mechanism used to reconcile the stressful experiences of moral distress in the moment and-then file away to process at a later date. Another sub-theme is that nurse participants noted that there are some nurses who are "just punching the clock" and "trying to get through the day". These nurses do not try to do their best on the job and do not seem to care as much, which nurse participants noticed and discussed.

\section{4.a. Surviving and Coping with the Impact on Personal Moral Integrity and Values}

Some nurse participants said that their values and personal moral integrity were impacted by the moral distress experienced in particular clinical situations in the SICU environment. Interestingly, some nurse participants chose to tell themselves specific things about stressful situations to reframe the events and help them cope with the job in the SICU environment. For example, a nurse (10) was distressed as a result of her work in the SICU explained her motivations to keep working and surviving: "Because I know that I could make a difference in at least one life. That if I can't save one, maybe I could save another" (\#13, p. 8). Moreover, another nurse participant (10) felt that it took nurses a while to mature into their professional role, discussing burnout and the role of nursing:

I think that and I know everyone calls it quote "burnout", but I think there's a certain level of nursing and most of the people that I run into - it's between like years three and five that they hit it the hardest, like one, two, and three they're still just like they try to just push, push $110 \%$, they're doing everything they can, but then I think after a few years you kind of get into your own groove and realize, like, I can't take all this stress home with me and I have to just really prioritize like what's important and if I push myself to the point that I'm 
exhausting myself every day that I work and I am staying for 14 hours a shift because of finishing up tasks and menial things that you know it's a 24-hour job. So it's like if I don't get to it, you know, someone else can get to it, but I think it's something that I think as you just progress in your career you learn to take care of yourself a little bit more and also include yourself, because it's like we tell our families, you know, I go home and sleep at night - if you're here 24-7, you're not able to take care of yourself, which means you certainly can't take care of them. I think it's something that it takes a few years to kind of get in your groove and even though you feel bad about it, you also know that if you're not taking care of yourself and if you're exhausting yourself doing all the little things that you, there's no way in hell that you're going to be able to take care of your patients. $(\# 10$, p. 11)

When asked how her personal moral integrity and values had changed from her years of experience in the SICU, the nurse (19) had this to say:

But, I mean, but as far as my morals and values, I still know that I deserve better, and they [the patients] deserve better so, you know, it's not like I'm, you know, I've changed my thought process to where, eh, this is enough. I still know it's not enough, but I've had to settle you know physical capabilities for a shift. Just what I can get done, I have to resign myself to knowing it's not possible to do everything, I morally know, I should be doing. So, I just kind of have to settle for doing less, but morally I still know that they [the patients] deserve more. Just do what you can. Just do the next one thing. Until the time's up. (\#19, p. 20-21) 
Interestingly, this same nurse (19) felt that it was her obligation to take less sick patients so that she may be used as a resource and also to ensure that all patients were taken care of by all nurses:

I think it varies, yeah, I think it varies shift-to-shift. Um. You know, it kind of sucks being in charge, because well at least for me, because I feel the obligation, you know, you can never take the sickies again. Even though the sickies would definitely benefit, because I have the most experience I can't take the sickies anymore, you know, I can't take busy ones, because I have to look out for everyone else and so sometimes I feel like people are like, 'God, [nurse] always assigns herself the easy s---.' But it's so I can keep an eye on everything else. Um. So I feel like when I've got an easier team, I can do a better job of keeping an eye out, but if it so happens that I have a busy one then-yes. You know, the other patients the other nurses, don't get as much help from me. Um. So I think it just varies shift-to-shift. Depending on what my, my patient load is and how much everyone else gets helped. (\#19, p. 21)

Interestingly, another nurse participant (9) who worked frequently in the SICU had the following to say about situations that create moral distress and impact ability to process grave situations:

Well, it's just at first I think it used to like kind of rock you and just kind of be like this isn't right, like I don't know, but then you know after a while I feel like you just get kind of callous and like hardened and just it's like it is what it is. I don't know coming into that environment when 3 [patients] was kind of the norm, to coming down to 2 patients it's, I don't know, it kind of really, prepared me. It's 
kind of really the, you know, trial-by- fire when I first started, but you know, I made it, so I don't know. I really don't get it. Things have to be going really, really bad really bad for me to get stressed out. And I think a lot of times, the big thing is I might even make the judgment call and say, oh this guy's a gonner, this guy's done, he's 'donezo,' he's not going to make it. You know, it's just like, I mean that's just how it is, you get an 87-year-old on a vent that's like, you know, just got destroyed by a car, you're just like, oh this guy's not going to make it - that's just how it is. You don't stress out about that, at least I don't anymore. Yeah, it doesn't even have to be about like being old either; if I have a, the big thing I see is ejected, if they are ejected or unrestrained, I'm just like, oh toast. This person's toast, man! (\#9, p. 6)

Interestingly, several nurse participants felt that they had been impacted by SICU nursing to the point that they recognized that it was impossible to do this work forever. Some nurse participants said that the moral distress they experienced did not impact their personal moral integrity and values. For example, one nurse (21) looked at things objectively and without a lot of emotion and had this to say about seeming to never get upset or bothered about his experiences in the SICU:

Well, it's kinda, I mean, I feel, like in that environment you should at least have an idea about what's going on. If you don't, you need to immediately like figure out what's going on. So. You know, I think that some people are just like, you really don't do that... or in a sense of anything, so at times...Um. But. Yeah. You know. It's just me. Like I think I do a better job of this especially with stress I do a better job of just kind of brushing it off forgetting about it. Even at home like 
my girlfriend's like, 'why are you not stressed about this, why aren't you thinking about that?' I'm like, 'I don't know'. (\#21, p. 12)

This nurse (21) went on to say that he does not think his personal moral integrity or values have been negatively impacted from his work in the SICU. According to this participant, he is thankful for what his job in this SICU has taught him about the fragility of life:

I would definitely say that since I've started as a nurse, like I would say, that I have definitely changed things. Like I'm much more caring. I would say so. Much more respectable to like- -life in general. I value life more. You know, because you've seen like how fast things can go south and how fast you can lose someone. (\#21, p. 31)

This nurse (21) feels that his work in the SICU has improved his personal moral integrity and values. He went on to say the following about how his job on the SICU has impacted his patient care and made him a better critical thinker:

I mean, if anything, it'd be for like the plus, like, you know, do more for what I can for the patients, I would say, because you know? There could be one day like, like one patient in particular, like recently. He broke his back, or broke his neck, I can't remember which one, um, but, you know? He, you know, was like worried he was going to die and then he kept asking me, and then he ended up dying a couple days—like a week later, he ended up dying just, because he had so much going on other than like what happened to him in the accident, like he had so many other co-morbidities that they finally, just kind of caught up to him, and you know he couldn't handle the situation, he couldn't handle the trauma, that 
happened to him his body just kind of started to shut down and then he ended up dying. Even though I reiterated to him, like, 'no, we're, you're, you know you're okay, we're gonna, we're gonna, we're gonna get you better, get you into rehab,' and all that stuff, and then he ended up dying like the next week. He just kind of went downhill, downhill, downhill. So. You know, just kind of like seeing things like that just kind of like, you don't know what's going to happen even under good circumstances. Even with good diagnosis you still think like things can go south really quickly. So. (\#21, p. 31-32)

When speaking about progression of one's nursing practice as a result of working on the SICU, another nurse participant (13) described her practice in this way: "I think it's a little bit of both, because my nursing practice is probably going to change, like be more assertive, cause I'm, I'm not just waiting for a physician's orders. I'm thinking ahead of what they're saying." (\#13, p. 3)

One nurse explained her approach to working in the SICU environment and dealing with stress, "When I leave the SICU, I try to leave it at the door." (\#13, p. 5)

Of interest with respect to coping and surviving the SICU is the exploration of how nurse participants are coping and what methods they use to cope with the stress of SICU. Nurse participants described the importance of coping and having a supportive group of nurses. Nurse participants discussed the importance and value of working with a good nursing team because it helped the attitude on the SICU and patient care. For example, this same nurse said that all the nurses on the unit banded together after a long code where ultimately the patient expired and post-mortem care was performed. This 
nurse went on to say that eating snacks and working with a supportive crew made all the work easier:

I don't think it would've happened if we had had like a different crew, or if we hadn't like_-it's just like a domino effect. Like between having a good day was working with good co-workers, we'd had like extra snacks and stuff and like, um, I had like, we'd been talking about Doritos, everybody had been talking about Doritos, like, all day long and so I grabbed like, I think I had like a 10 dollar, 10 dollar bill in my pocket and [nurse] was gonna go downstairs, because she wanted a chocolate bar so I was like, hey, get a big bag of Doritos, like we need some chips. And so like it was just, we'd all been in a good mood, so that when my patient died we were able to kind of transfer that good mood and just kind of... ahhh... like just have like a chill experience with it, if that makes sense. (\#2, p. 49-50)

This nurse (2) also noted that it was difficult to work with some nurses, because these are situations that are non-modifiable from her perspective, as she described:

But, what about when we do take steps to change them preventatively? Like there are days when, you're just like, hmm... I'm not working with a crew I really like all that much. I'm gonna take a f------ massive snack to share with everybody. Like, I love [nurse] to bits and pieces, but she is hard to work with. (\#2, p. 48) One nurse participant described herself as being resilient. Other nurse participants noted the importance of working together. Nurse participants were able to work through the emotional and stressful work of the SICU by recognizing they are patient advocates working together. Nurse participants noted being invigorated by patients' outlooks and 
feeding off of their emotions. Interestingly, nurse participants were able to identify the stress of the job, but also the fact that they loved working with patients in SICU. Another nurse participant discussed ways of preventing moral distress by identifying an emotional co-worker sensing when she needed to go outside for a cigarette break and suggests this in anticipation of her peer having an emotional outburst. Thus, this nurse participant said she does this for the well-being of everyone in the SICU to prevent emotional outbursts that may impact patient care through aggression and over-reaction.

Nurse participants managed to cope in a variety of ways. Some nurse participants reported that they smoked cigarettes, drank alcohol, slept more, exercised more, and ate to excess. Interestingly, nurse participants discussed communicating with their loved ones as a form of coping. One nurse participant (9) went on to say the following about how their care was impacted by the job and what they did to cope:

Well talking with other nurses is the best 'cause they like know the s--- and so it's like you can really joke around with them and stuff. Drinking definitely. And I'll switch it up sometimes three [shifts] in a row is too much and I have to, I'll be like, alright, this next schedule I'll do a couple days on and a couple days off. I used to do 8 in a row. Six -6 to 8 in a row. Yeah man, 'cause there hit a point when I was just like I don't, I don't like my patient anymore, like, I don't like this guy, I don't like him and it can impact your, care at that point. (\#9, p. 18) Many nurse participants found that exercising, playing golf, cycling, spending time with friends and family, and decompressing with co-workers were helpful ways to cope. One nurse participant described a "hate letter" she was "writing" to the CEO and would submit it if she were ever fired. Other nurse participants loved to manage their stress by 
reading. Participants noted spontaneous potluck pitch-ins with homemade food brought into the unit that helped to boost morale for the day. One nurse participant (11) said the following about his personal coping method with the moral distress at work:

"Well, when I get home, it's fear - when I get through the door I forget about it. Sometimes I'll have a couple beers." (\#11, p. 8)

One nurse (12) felt that she was repressing her moral distress, thus she was compartmentalizing it and then dealing with the situation after work in her own personal time. This particular nurse was coping by completely blocking the events out of her mind. For example, this particular nurse explained of how she copes with moral distress experienced through situations in the SICU environment, as follows:

I think that I'm... and I really haven't thought about it until just now, but I think that, you know, I've never been able to recall patient's names, maybe some of them occasionally, or even like when you ask me for a specific situation, I couldn't think of one, there's thousands [laughs] and I think that I'm like literally just shutting them off like when I leave there, I'm not going to think about them again unless I deal with them that night, you know what I mean? That is weird, because I've never thought about it before but that might be why I don't remember... (\#12, p. 29)

This nurse (12) went on to say that she has a great support system of friends and family to cope with the clinical experiences at work. Interestingly, she also said that she would not know where she would be if she did not have such a strong support system:

But I think if I didn't have those resources, I don't know what the f--- I would do. I mean, I know, the like, uh... like, progression of, you know, some people- 
addiction and drinking and sex or whatever it is that you do to cope, um, and then depending on your personality like I know where that goes but... if I didn't have resources I don't know what I would be doing. I'm obviously not doing it... as much as I could be with those resources. (\#12, p. 32)

When asked how she copes with all the clinical experiences she encounters in the SICU environment, another nurse (19) had this to say: "My cats. [laughs]" (\#19, p. 21). A nurse (17) on the same unit when asked about how she copes with stress and the morally distressing clinical situations at work when she is at home, she said the following: But, then like who really wants to f------ talk about it, except maybe other nurses who can relate. I would say I like vigorous exercise, like I have to do something to like get my heart rate above 180 beats per minute at least three times a week for me to like be able to be cool as a cucumber at work like, because otherwise, like I might feel like exercise sounds super generic and then the other thing, too, is like I really firmly believe in like spending at least 10 minutes of silence every day like that's something, I like hold sacred to me. Um. I think in part, because like life is loud and like in every sense like you may not even notice it. And then I also like, put my physical feet on the ground like, outside I don't care if it snows I don't give a f--- what the weather is like, I'm all about grounding, like touching the literal Earth it's like, and I think that like keeps you—keeps me—rather intune with like, the world's still f------ turning dawg, like you can't like get, because I'm like somebody who can get really like hung up on a moment like, and I don't try to like obviously, it's not like a quality that I like necessarily about 
myself, but like I can get really hung up on something, and like I pick it a part and obsess about it. (\#17, p. 13).

Another nurse participant (12) described how she copes:

Because people don't get it so. Going out with people from work or talking to people from work if something crazy happens where you know I talk to people on the way home after a shift. Like a really good thing that was happening when we 1st worked there and even [friend at work's name] was there then. At the end of that. We would have these f------cowboy rodeo nights that were just f------ insane and we would go in the doc box and chart and we would be there forever and it wouldn't even, like sometimes we weren't even always charting the entire time, it was literally us just talking about what the f--- just happened and like going back

over little details that we couldn't say in the moment, and you know talking about the doctors and the decisions that were made and the craziest families and whatever. And I mean we would stay there sometimes until like 8:00 AM, 8:30 AM or well 9:00 AM. (\#12, p. 7)

\section{4.b. Compartmentalization}

Interesting, female nurses were better able to cope with the emotional aspect of moral distress and attempted to process and define it so that they would understand it better. For example, one nurse (10) explained that her compartmentalization was a positive form of coping and professional development and discussed her expertise:

Mine is definitely more the back end of it, it's not the front end of it, the nursing care. That's stressful, but it's, again we all go back to like, you know, most of these people have never been in significantly stressful situations like this - this is 
the biggest stressor they've ever had in their life. They woke up, going to work today, like instead, they were in a massive car accident, or they went, you know, they were driving home from church on a Sunday or something and they had a heart attack or something big like that, that caused them a major stress. So understandably they're in a stressful situation. So, not everybody copes with stress the same way, obviously, and when it's thrown at you all of a sudden, it's usually a fight-or-flight, either people shut down and close or they just like retaliate back and just regurgitate that stress back on someone else. So, as much as we can get pissed at them, you know, I think for the most part I feel like I have a pretty good handle on, like, pulling that back in and just kind of like understanding like, yeah, I can get pissed, I can vent about it, I can bitch about it for a minute, but ultimately it's not going to like make me quit my job because that's just part of the job. (\#10, p. 14)

Furthermore, she went on to say the following about compartmentalization, specifically, which is more intuitive and self-aware than the male nurses' responses, which will be described later:

I think for me, I think, it's just that you, I have to just compartmentalize - it's not that I don't feel it's important still or that. It's not that I don't. It's not that I don't think it's important or anything like that, but I think it's also a way you just have to prioritize it to a point that if it's not something that's in my control to change right now. (\#10, p. 22)

Some nurse participants visited patients in rehab or in other areas of the hospital postSICU discharge in order to follow-up on the condition of their former patients. This 
provided a sense of closure for some, but may be viewed by other nurses as an inappropriate relationship to have with a patient.

Male nurse participants were more likely to compartmentalize moral components of care after the end of shift when they were not working. In reference to a nurse participant, he reported that he coped through drinking a couple of beers. He had the following to say about a particular event at work that bothered him and interestingly, it occurred after work:

"The first guy that ever died on me he was an alcoholic he was DNR/DNI. And he had a lot of those what are those called those bandings? Varices. He was awake. Like not intubated or anything. And like at 7 in the morning during shift change, it was the month when new residents started on the unit. I remember [named date] because a whole new team came in. His bandings burst and he was just drowning in his own blood. And the guy was drowning in his own blood. And I was putting a red rubber down and trying to maybe suction it, but you know you can't, we're not going to intubate him, we can't do anything with him. So. I went and got some morphine and Ativan and, and then I was just trying to and then all of a sudden he's dead. He just drowned in his own blood. And I was like oh that's f---- up. Yeah, but like [one nurse's name] and [another nurse's name] like we weren't, like they knew, they could tell I was frazzled. Like it f----- me up because I was thinking about it I just like, just all the blood coming out of his mouth. You know? They, they knew that I was that, this was right at shift change. So it was like right there. But then I was like pissed. Then I was worried that I forgot to chart the morphine I pushed 'em in before he died. (\#11, p. 8-9) 
Moreover, male nurses were more likely to think about the intricacies of the clinical situation at home after work and focused on reacting and caring for the patient the moment the events were unfolding in the clinical setting. For example, one male nurse in graduate school had this to say about compartmentalization: "Some things, like, we just have to compartmentalize and just do your job because almost, like, there's kind of a legal fear, you know, like you, like you, if you don't do it as you were told, you know" (11, p. 3)?

\section{4.c. Nurses "just punching clock"}

Nurse participants were quick to note that some nurses are "just punching a clock" and are not interested in caring for the patient to the best of their ability. One nurse participant (2) noted her frustration with those nurses that do not care as much as she does:

There are certain nurses that that they go in and they punch the clock they do their job and they do a damn good job but they don't get invested and they don't like look at for instance [names a specific nurse]. He's a fantastic nurse he doesn't work that often it's easy for him to not get invested. It's rare that he'll take care of the same patient twice even in one week. So there's not there's not enough time and there's not enough energy invested in those families for him to really give a s--. He cares about his patients you know he notices when something is going wrong he's an excellent nurse he's an exceptional like he's an exceptional nurse. Would I want him to care for my loved ones? Probably not. But he's a great nurse. It's just I feel like some of the nurses that we work with don't. They're just 
punching a clock. And I just it's supposed to be the caring profession. And I get it. It's hard. We work in a hard area. (\#2, p. 3-4).

Some nurse participants felt that they had changed personally since taking the job in the SICU and that their families noticed a change. For example, one nurse (5) noted that her family had noticed a change, but the participant felt it was from working nightshift and not from working the job itself in the SICU:

[laughs]. It, it's not really. I don't really know how to describe it, it's just, it takes a lot out of you. I do know that for sure. And like a lot of times like since being on like nightshifts, but like the experience that I have, my family could like tell a difference of like how much I've changed as a person - just working from nights. Like, they're just like you're not as happy as you were when, you know, you were on orientation on days. I'd miss out on a lot of opportunity like a lot of things like family- and friends-wise because I'm trying to catch up on sleep to go back in to take care of patients like that -just a lot more negative. I feel I just don't get like quality sleep like I did when I first worked days. (\#5, p. 3-4)

Some nurse participants felt that their quality of care was impacted by how the job had impacted their ability to deal with issues such as death and dying. For example, one nurse (12) had this to say about perpetuating suffering at the end-of-life in patients, particularly when the prognosis is poor:

[takes a deep breath]. I mean, I feel like, I've gotten so used to it, but I don't really have feelings about it anymore. Um. It's changed my thinking, in the fact, that there's s--- and it's every situation, not just in the hospital, there's always s--going on in the background that isn't right, that people know isn't right, but it still 
continues, um, and it's changed, it's kind of, I guess - jaded - is the word maybe, that comes to mind with that. Um. I think it's, and it frustrates me to say it, but it does like, make me feel like, I can't change it so almost just giving up to it like, the only thing that I keep, the only thing that I keep for myself is the fact that I'll go in there and talk to 'em [the family and patient] still like they matter, but then I'm in the background, you know, refilling the drips and, uh, telling the family if they call on the phone, you know, yes, they're here nothing bad is going on [laughs] you know, what I mean? (\#12, p. 11-12)

Nurse participants noted that since working in SICU, they had developed an ability to focus on the bigger clinical picture of patient care, rather than fretting over minute details. For example, one nurse (6) said the following:

No. I mean, I-I, used to always like, I would stay and try and get every possible task done, because that's what's good for the patient and now I'm like, oops, sorry, they didn't get this, this, and this done, but, I'm done working like, I'm just for now so I don't like want to stay over because it's not, it's not gonna change. I don't know. I mean, I guess it's going to change the patient's outcome, maybe but it doesn't feel like it's going to. And it's not like my additional hour of work is going to make that big of a difference in that person's life. $(6, \mathrm{p} .15)$

\section{Theme 5 - Moral Crescendo's Coup De Grâce - Suffering and Wounded-“Cannot}

\section{Take It Anymore"}

The fifth theme represents the final part of the moral distress equation, which is the complexity of the SICU environment and an inability to establish an outlet for stress over time that may potentially lead to a moral crescendo. In this study, one nurse 
identified as being "burned out". Moral Crescendo occurs when moral residue has builtup to the point of being ignored, and, in this study, the crescendo is the manifestation of this response that the nurse "cannot take it anymore". Thus, moral crescendo's coup de grâce or death blow to the mental and psychological suffering and wounded nurse is to either move on or seek treatment.

The fifth theme represents the final part of the moral distress process model, which is the complexity of the SICU environment and an inability to establish an outlet for stress over time that may potentially lead to moral crescendo. Theme 5 is comprised of two sub-themes, including: (a) Burnout; and (b) Can't take it anymore - ready to leave. All of the SICU nurses named an experience that was morally distressing to them. This event was either morally distressing by itself or in the context of other experiences that were similar making nurses question the ethics of their nursing practice and their own personal moral views of right and wrong. Interestingly, some nurses were able to compartmentalize easily and move on from the moral distress generated by an ICU patient care position; but other nurses were challenged by "burnout" and "can't take it anymore".

\section{5.a. Burnout}

The complexity of the SICU environment and the severe acuity of the patients, the stress experienced by nurses, and the inability to establish an outlet to relieve stress over time leads to "burnout", possible PTSD, and nurses quitting their position in the SICU. Thus, moral crescendo's death blow to the mentally and psychologically suffering wounded nurse is to move on, quit, or to seek psychiatric treatment. For example, as one 
nurse participant described herself as being "burned out" and ready for a slower-paced job because patient care in the SICU was wearing her down:

Yeah. I mean, I've said like, specifically, like, I think that I'm so burnt out that it's affecting my patient care and they will say, no I feel like your patient care is the same. I don't think it's affected you. (\#6 p. 18)

To reduce her stress level, this nurse participant has since left her job in the SICU. Moreover, several other nurse participants noted that it would be beneficial to take vacation time to prevent getting "burned out" from working in the SICU environment. For example, one nurse participant (11) said the following:

You know what would probably help? And we've talked, touched on it a little bit. Maybe some forced vacation time not forced but like, you have to, you have to do a certain amount of weekends and then you have to do a certain amount of weekends off. You know? Clear your head. Perspective. Like keep you from getting burned out.

(\#11, p. 38)

Furthermore, another nurse (8) participant explained why he decided to leave the bedside in the SICU:

So, I've watched them do it for five years, so like I basically was an intern, you know. I, I know what the job is, so like watching every day drove me to it. The autonomy. The hours. The pay. Um. The respect. Are really probably like the main ones. And it's not the driving factor for me, but you know, being able to triple my salary, like I mean, I grew up poor. They say money can't buy happiness, but it can get you pretty close, like, if I can make enough money in my 
work life to keep my personal life going that's a huge factor for me. Would I stay a bedside nurse? No, because then the other ones come into play. Autonomy and then the hours and the respect. Hours is a very person-specific I think. And I had this discussion the other day because the pro of nursing is, you know, you can work three days a week and be off for four, but I think that's also a con in nursing, 'cause if you've ever done a run of three in a row you've never hated yourself or your job more than when you're in the middle. (\#8, p. 10-11)

\section{5.b. Cannot Take it Anymore - Ready to Leave}

One nurse participant (12) decided she was ready to leave the SICU environment due to the moral distress. Interestingly, she refused to participate in the required peer chart audits and said she called in "sick" frequently. She explained why she decided to arrive at this mindset of not caring about her job anymore and feeling ready to leave the SICU environment:

And I really, like, wanted to use my nursing skills if you, if I bypassed all that... I wouldn't have that like clinical, that type of clinical experience. [Nurse friend on the unit] going back was huge. Her saying hey let's f------ do it and I'm like why not do it with somebody that you know I can experience and go through with it will be easier that way. That was a huge thing for deciding at the time to go back. But it was always in the back of my head and then every single year that passes there, you just get more and more fed up, so that was definitely part of it. I think it was just a bad like year we lost a lot of good people and it wasn't the same there anymore and it was a lot harder to deal with the bulls---, so it was like, okay just get started and get the process rolling - I'm not ready to quit yet, but by the time I 
put in another 5 years I'll be ready. [laughs]. Especially in something in particular like when you said like signing out and not giving a f---. Especially when you're passing it to another nurse and like they're just - they don't give a f---. [laughs]. What's happening and you've put everything into this patient and you've done things and you're trying to it made me like I was attached to the family or whatever and you're trying to make them care that like or I don't know it's just. Getting out of there and just signing out. Yeah. Absolutely that resonates. [laughs]. (\#12, p. 13-14)

It is important to understand the sheer emotional and psychological distress that nurses experience when morally distressed. To further understand this nurse's distress and despair, it is important to understand her reasons for quitting. For example, this nurse refused to scan medications and use the BioVigil hand washing device to monitor each nurse's hand washing practices. This nurse's (12) despair is illustrated by the following: Of the people that we work with, we all acknowledge that like I'll never get mad at you for calling-in and I don't care if I work with 2 people. How far does it have to go before I get fed up and yell at everybody in the room that we're torturing somebody [laughs] you know what I mean? I think that either it's building up and something small will set me off or it's just has to be really really, really, really, bad for me to f------, for me to say like no f------ way [laughs], you know and that's different. (\#12, p. 23-26)

\section{Summary}

The purpose of this descriptive qualitative study was to explore nurses' perceptions and experiences of moral distress and moral residue in the adult SICU 
environment in order to determine root causes of moral distress, as well as perceptions and experiences associated with moral distress, moral residue, stress and coping, and perceived quality of patient care. By exploring these concepts among SICU nurses, moral issues that lead to moral distress were identified. Five themes described the process of moral distress and broadly include concepts such as caring, advocating for patients, power differential, surviving moral distress in the SICU environment, coping with moral residue, and a subsequent crescendo effect from the pile-up of stress. Interestingly, these themes illustrate a broader process of moral distress. Through the analysis of the interviews, he following care-related issues were identified that led to moral distress among nurses in the SICU environment: (a) communication with families, patients, nurses, physicians, and other healthcare team members; (b) end-of-life patient care issues where suffering is caused to the patient and inner turmoil rocks the nurse; (c) problems with nursing management that include lack of support, short staffing, and lack of resources; (d) working with incompetent nurses with a lack of SICU experience; and the demands of additional charting through peer chart audit and other related responsibilities. Interestingly, these care-related issues were a result of exploring SICU nurses' perceptions and experiences of not only moral distress, but also moral residue, and in two cases - moral crescendo.

Overall, these findings suggest that moral distress is a problem that plagues nurses in the SICU environment. The root cause of moral distress in the SICU environment was explored with the five emerging themes that highlighted the nurses' morally distressing experiences. More specifically, these experiences were synonymous with end-of-life events and clinical situations with ethical issues when quality of patient care was 
prevented and marked by the inability of nurses to "do the right thing". Thus, the results of this study exemplify the process of moral distress when caring for patients in the SICU environment leading to moral residue among SICU nurses over time, and culminating in moral crescendo. From these results, a better understanding of the phenomenon was achieved leading to insights essential to the development of future interventions to address moral distress and moral residue among nurses, while improving the quality of patient care in the SICU environment. 


\section{CHAPTER 5 DISCUSSION}

This descriptive qualitative study explored nurses' perceptions and experiences of moral distress, moral residue, and moral crescendo in the adult SICU environment in order to determine root causes of moral distress; as well as nurses' perceptions and experiences associated with moral distress, stress and coping, and perceived quality of patient care. By exploring the phenomenon of moral distress as described in the five identified themes, a better understanding of how nurses' develop moral distress in the SICU environment and cope with the stress and complexity of SICU patients has been obtained. Moreover, the interviews highlighted the process surrounding the development of moral distress, moral residue, and moral crescendo among nurses in the SICU environment. These insights are essential to the development of future interventions to address moral distress, moral residue, and moral crescendo among SICU nurses to provide strategies to manage stress, prevent burnout, and improve quality patient care.

Before discussing the significance of this study's results with the extant literature, it is necessary to reiterate the identified themes. Five themes of moral distress were identified from the 21 nurse participant interviews of this study: (A) Theme 1 Concentrated Caring in High Stress Environment—- "Doing Your Best” comprised of the following sub-themes: (a) "doing your best” for the patient; (b) caring; (c) communicating with families of patients near the end-of-life; and (d) suffering and inner 
turmoil; (B) Theme 2 - Advocating for Patients \& "Doing the Right Thing" comprised of the following sub-themes: (a) physician-nurse power differential; (b) patient advocacy; and (c) positive nursing - "doing the right thing"; (C) Theme 3 - Surviving Moral Distress in the SICU Environment-Magnifying Stress \& Self-Preservation with the following sub-themes: (a) nursing management problems, short staffing, and lack of resources; (b) incompetent RNs lacking in SICU experience; (c) demands of additional charting and responsibilities. Nurse participants revealed that the SICU environment magnified the experience of stress. As a result, these nurses had to use self-preservation to not only continue caring for their sick patients, but also to protect their own wellbeing, because the healthcare organization did not advocate for nurses to stay at the bedside; (D) Theme 4 - The Stain of Moral Residue - Surviving \& Coping with the Impact on Integrity \& Values, with following sub-themes: (a) surviving and coping with the impact on personal moral integrity and values; (b) compartmentalization; and (c) nurses “just punching clock"; and (E) Theme 5 - Moral Crescendo's Coup De GrâceSuffering and Wounded - "Cannot Take it Anymore" with two sub-themes: (a) burnout; and (b) cannot take it anymore - ready to leave. Additionally, these identified themes illustrate a broader process of moral distress, moral residue, and moral crescendo that was unexpected, but indicative of the damage that moral distress wreaks on caring and compassionate SICU nurse participants, and further provides evidence of their ability to survive and self-preserve in such a caustic, traumatic, and hectic environment where lives hang in the balance on a daily basis.

\section{A Practical Definition of Moral Distress_-Violations to Core Values \& Duties}


Important to the concept of moral distress is that the nurse knows what ought to be done, but is prevented from doing so. A motivation for conducting this study was to clarify a practical concept of moral distress that coincided with a qualitative descriptive approach, which is a factual description of an event in a participant's own words through an exploration of the who, what, and where of events (Sandelowski, 2000). Furthermore, according to Sandelowski (2000), the expected outcome of a qualitative descriptive study is a descriptive summary of the informational contents of data organized in a way that best fits the data. Thus, the important aspect of this study is refinement and clarification of a concept that results from a violation of the nurse's core values and professional duties. Moral distress stems from inaction or action on the part of the nurse not to effectuate change in the perceived right direction of some particular clinical situation. According to Epstein et al. (2016), nurses that work in the ICU are more likely to experience moral distress because of the high stress environment in which they work. Nurse participants in this study felt that their inactions or actions were part of a broader unethical model of care where these occurrences were allowed to perpetuate.

This study is important because it refined the definition of moral distress by 21 SICU nurse participants reinforcing the importance that the phenomenon is based on a core violation of one's moral values and professional duties as a nurse. There is a need for clarification in moral distress research because although this phenomenon has been studied since the term was first coined by Andrew Jameton in 1984 and in over 30 years of investigation, no intervention study aimed at acknowledging and addressing the impact of moral distress at the unit, team, and system-level has been conducted. For example, Campbell, Ulrich, and Grady (2016) suggest a broader understanding of moral distress. 
The authors argued that this taxonomy of moral distress includes more experiences, such as moral undesirability and allows for new lines of thinking about how to deal with moral distress, which would be beneficial in creating solutions (Campbell et al., 2016). In this study, the nurse participants' perceptions and experiences with numerous patient care situations in a Level 1 trauma SICU were not morally undesirable or based on an unfortunate patient care outcome. Instead, the nurse participants' perceptions were morally distressing and stained in the form of moral residue due to the severe violation of core moral values and professional duties as a nurse that were noted across the all nurse participant interviews. Thus, broadening the expanse of the definition of moral distress to include moral undesirability dilutes and waters down what the concept of moral distress is truly about - that is, a moral wrong occurring in patient care and a violation of a nurse's core moral values and professional duties in the process. According to Epstein, Hurst, Mahanes, Marshall, and Hamric (2016), broadening the definition of moral distress, as Campbell et al. (2016) have suggested, will not offer any benefit and it may weaken the argument for further study. According to Webster and Baylis (2000), moral distress is defined as when one fails to do what is right as a result of a judgment error, personal failing, or circumstances beyond their control. Interestingly, Hamric et al. (2012), supports this definition by naming internal constraints of a nurse as potential root cause of moral distress and includes perceived powerlessness, self-doubt, and lack of assertiveness.

Thus, morally-distressing experiences are marked by the nurse knowing that the right course of action is being ignored. This is key because the nurse recognizes the violation to their core values and duties as a nurse. However, as the nurse participants in 
this study demonstrated, just because a nurse does not know the proper chain of command in reporting ethical issues at the bedside, does not make them a "bad" nurse. Instead, nurse participants bemoaned not being aware of or educated on the proper way of handling ethical issues that occur at the bedside. Thus, nurse participants in this study voiced concerns over this lack of knowledge in the SICU.

In this study, each of the 21 nurse participants recounted vivid perceptions and often traumatic experiences with moral distress; damaging unresolved moral distress in the form of moral residue stained these nurse participants; and the crescendo effect forever changed two nurse participants. This evidence alone warrants further study, particularly regarding how to acknowledge and address moral distress and moral residue at the bedside more aggressively. Additionally, distributing a call to form committees and collaborate with healthcare organizations, administration, and stakeholders in creatively working with ICUs, directors, and managers to promote individual nursing communication about ethical issues and assessment of nurses' well-being and health as it applies in assessing the traumatic experiences generated as a result of the care they are providing and certainly take home with them — as is evidenced by the results of this study. This study confirms findings by Epstein et al. (2016), with respect to moral distress research, that it is necessary to take the next step in acknowledging and addressing moral distress and moral residue in healthcare, because further re-definitions that do not focus on compromised moral integrity risk conceptual confusion that will further slow research progress.

According to Epstein et al. (2016), research funding is competitive and diluting the concept by broadening the focus of moral distress to include other characteristics will 
only make interventions aimed at acknowledging and addressing the concept of moral distress more difficult to develop. Moral distress, to the lay observer, may seem similar to other concepts, such as compassion fatigue, burnout, emotional distress (Epstein \& Hamric, 2009) — but moral distress as this study revealed, is not. Rather, moral distress is characterized by a responsibility to the patient's well-being that is compromised by powerlessness in the face of violations to their core values and duties as a nurse. Previous research stated the importance of investigating the temporal, contextual, and situational specific factors related to moral distress (Dudzinski, 2016; Epstein \& Hamric, 2009; Hamric, 2014; Hamric et al., 2012). Interestingly, the nurse participants in this study provided rich descriptions of situations and contexts in the SICU environment. However, it was difficult to explore how time impacted the unraveling of the nurse's moral residue into the crescendo effect, because the experience is difficult, unique, and complex for each nurse as this study demonstrated. For example, nurse participants in this study were able to recount specific clinical situations that were morally distressing, which is also indicative of moral residue — due to the quick recollection of traumatic events that conjure up emotional reactions as suggested by Epstein and Hamric (2009).

According to Epstein et al. (2016), a broader definition of moral distress is undesirable, because a focused and practical conceptual definition is needed. For example, Jameton (1984) coined the term moral distress over 30 years ago and there has still yet to be a large-scale intervention study aimed at mitigating the effects of moral distress and moral residue at the organizational, unit, and individual level. The results of this study indicate that nurses believe that the healthcare organization where this study was conducted is not supporting the well-being of nurses, due to administration and 
management's lack of action to provide the appropriate resources — similar to other hospitals nationwide that still need to acknowledge and address moral distress. Epstein et al. (2016) noted that the lack of an established effort to address moral distress in the healthcare setting is a result of adding to the definition characteristics that are not morally distressing. It would be impossible to develop interventions to address moral distress if the definition were broadened to encapsulate concerns of mild distress or moral uncertainty, because this would dilute the concept of moral distress itself and prevent a focus on the true problem - a moral wrong is occurring in the care of the patient and the nurse is unable to change or influence the outcome in the patient's favor. Thus, the execution of a moral wrong is why this research is important. If moral wrongs are perpetuated at the bedside, then it would make sense to pinpoint and highlight the impetus for those patient care situations, thus the significance and goal of this study.

A narrow definition of moral distress that includes a violation of core values and duties is warranted and should not be broadened to include moral undesirability, as previous research has suggested (Campbell et al., 2016), because it would be demeaning to the nurse participants in this study. According to Epstein et al. (2016), an undesirable broadening of the definition of moral distress creates obfuscation in understanding the concept for practical purposes of addressing moral distress in the clinical setting. For example, as members who sit on active ethics and moral distress consultation services, Epstein et al. (2016) recommended a practical definition (i.e., violation of one's core values and duties (Epstein \& Hamric, 2009), because when moral distress is respected as a real phenomenon, then healthcare organizational leaders and stakeholders are more likely to engage with healthcare staff to acknowledge, address, and create solutions. This 
study acknowledged that moral distress is not only a real problem for healthcare organizations, but also moral residue is damaging bedside SICU nurses at tremendous rates as all nurse participants demonstrated moral residue in their interviews and if left unresolved and unchecked, progressed to the crescendo effect.

\section{Identified Root Causes/Sources of Moral Distress and Moral Residue}

The root causes of moral distress that contributed to a crescendo effect of moral distress with respect to time (e.g., years of exposure), frequency (e.g., how often in their nursing career), and intensity (e.g., severity of the experience) of exposure in the SICU nurses. To the best of this author's knowledge, it is unclear if exploration of two actual experiences of moral crescendo have been observed and described in the research setting. Specifically, moral crescendo was demonstrated by two SICU nurses who had to seek treatment related to patient care experiences in the SICU. Theme 5 - moral crescendo's coup de grâce—-suffering and wounded: "cannot take it anymore", identified two subthemes: burnout and "can't take it anymore - ready to leave". Based on these results, it is clear that nurses who experience a crescendo are "burned out" and experiencing psychological turmoil related to their patient care experiences. Being "burned out" is significant because the individual nurses withdraw from the ethical dimensions of practice as reported (Epstein \& Hamric, 2009).

Regarding the two nurses that experienced a crescendo effect, the root causes that contributed to their experience of moral distress involved end-of-life care. Interestingly, based on these two nurse participants, this was not the first morally distressing event these nurses had encountered. Both of these nurses had more than a year of SICU experience and both described frequent occurrence of morally distressing events, events 
that occurred on a weekly, sometimes daily basis. It was difficult to determine the intensity of the morally distressing patient care experiences that led to unresolved moral residue and the subsequent crescendo effect. The intensity of the morally distressing patient care experience was exemplified by the nurse participants' vivid accounts whereupon they readily recalled specific names, dates, times, procedures, and events in emotionally gripping detail; thus, these rich descriptions provide evidence for how intensely bothersome these morally distressing experiences were for the SICU nurse participants.

The nurse participants in this study described moral distress and moral residue that violated and continued to violate their professional values and duties. Previous research demonstrated the negative consequences of moral distress through the symptoms characterized by frustration, guilt, physical symptoms, and anxiety related to repressed and unaddressed violations of core values and duties (Austin et al., 2005; Gutierrez, 2005). Interestingly, these nurse participants were also psychologically and emotionally distressed by their moral distress, which was evident in that they were able to recall specific dates, times, patients, names, and faces in their stories. In difficult clinical situations, researchers are warned to be alert for strong emotional reactions because this may be a potential indicator of the crescendo effect (Hamric \& Epstein, 2009).

All of the nurse participants in this study exhibited a great deal of psychological distress, particularly the two nurses who had actually experienced a crescendo effect from patient care experiences related to end-of-life care. To the best of this author's knowledge the crescendo effect is a particular novel aspect of this study-documentation of the crescendo effect in the scientific literature, which acknowledges the experience and will 
provide more of a focus on future research aimed at addressing moral residue and the crescendo effect. According to Epstein and Hamric (2009), moral distress is not only morally distressing due to the ethical components of the experience, but it is psychologically distressing. But Epstein and Hamric (2009) appropriately clarify that psychological distress describes how an individual responds emotionally to clinical situations, but does not guarantee a violation of core values and duties. For example, Epstein and Hamric (2009) described several examples of psychological distress including: situations of short-staffing, clinical situations where a healthcare provider must prioritize care, or patient care events where the patient's condition deteriorated and the family could not be reached. Importantly, Epstein and Hamric (2009) noted that these clinical situations did not challenge or violate core values, but instead, manifested as psychological distress. However, in order to be classified as morally distressing, one's core moral values, and professional and personal duties must be violated (Epstein \& Hamric, 2009).

Arguably, nurse participants in this study cited short-staffing as a major root cause of moral distress, because not having enough nurses in the SICU during nightshift prevented adequate and safe care, particularly for new, less experienced nurses. Thus, short-staffing itself may not be correctly classified as morally distressing as Epstein and Hamric (2009) importantly pointed out. However, results of this study indicated that nurse participants revealed that short-staffing on nightshift in the SICU in particular led to a violation of nurses' core values and duties due to unethical care that was allowed to persist as a result of not having enough staff. Furthermore, short-staffing contributed to many nurse participants in this study experiencing moral residue related to care, because 
they described vivid, traumatic events where they had to work short-staffed and it impacted the quality of care provided on the unit. Furthermore, these nurse participants assumed they would not be able to provide care during their nightshift on subsequent nightshifts, because working with four nurses on a 10-bed unit became the norm. This corroborates what Epstein and Hamric (2009) noted about citing evidence for the presence of moral residue, such as changes in personal or professional roles that are subsequently consistent with the loss of moral integrity.

Nurse participants in this study survived moral distress in the SICU environment through self-preservation. However, this period of time that Jameton (1984) coined as the initial distress of moral distress is the period of time when stress seemed to be most magnified for the nurse participants, which occurred as the event is unfolding. Interestingly, the nurse participants re-told clinical situations that challenged their core values and duties. These clinical challenges included nursing management problems, short staffing, lack of resources, working with incompetent RNs lacking in SICU experience, and the demands of additional charting and other unit-related responsibilities. Regarding the demands of additional charting and other unit-related responsibilities, the nurse participants felt were preventing them from providing their best quality of patient care. The nurses' perceptions were that these feelings and events were left unresolved in the nurse participants' minds, just as they were when the nurse participants initially had these experiences. Furthermore, it is evident that what Jameton coined (1984) as reactive distress — or moral residue — is the unresolved feelings that manifest as the personal and professional roles changes that Hamric and Epstein (2009) warned are indicative of loss of moral integrity, which nurse participants in this study exhibited. For example, nurse 
participants described a variety of coping, but some nurses felt that they did not try their best anymore-like when they initially started working in the SICU. Thus, the repeated exposure to patient care situations that are morally distressing resulted in nurses not trying their best.

Interestingly, what constitutes a root cause of moral distress has been parsed out into three different major root causes from previous literature. This study confirmed the root causes of moral distress explored in the creation of the Moral Distress Scale-Revised (MDS-R) by Hamric, Borchers, and Epstein (2012) and extended the acknowledgment of moral distress through communication, which was therapeutic and further addressed root causes. For example, Hamric et al. (2012) created the Moral Distress Scale-Revised (MDS-R) in an effort to more accurately capture the quantitative frequency and intensity of moral distress among multiple healthcare professionals across multiple settings. Thus, the MDS-R was not created just for critical care nurses, but rather to capture a broader audience of nurses and other healthcare professionals who are potentially suffering from unresolved moral distress across diverse healthcare settings (i.e., ICU and non-ICU) (Hamric et al., 2012). From this instrument development and validation, Hamric et al. (2012) identified three root causes of moral distress that have been identified in the subsequent literature: a) clinical situations; b) internal constraints; and c) external constraints that are characterized by negative feelings, powerlessness, conflicting loyalties, and uncertainty (Dudzinski, 2016; Hamric et al., 2012; McCarthy \& Gastmans, 2015; Russell, 2012; Savel \& Munro, 2015).

This study supported the three root causes of moral distress (i.e., clinical situations, internal constraints, and external constraints) identified by Hamric et al. 
(2012). For example, this study identified similar root causes for clinical situations, such as primarily encompassing issues related to unnecessary or futile treatment. However, this study extended this pool of potential root causes by identifying nurses that find positivity in their experience of moral distress. For example, some nurses saw their experiences with patients near the end-of-life as an opportunity to do their best in an environment that does not always support their ability to operate at maximum ethical sufficiency in considering values and duties to the profession of nursing. Moreover, this study supported the perceived powerlessness and self-doubt accompanied with violations to one's core values and duties as a nurse. However, this study extended this internal constraint by identifying the lack of communication and de-briefing that is occurring and, thus, contributing to increased experiences of moral distress and moral residue. Finally, this study supported a primary external constraint noted by Hamric et al. (2012), which is the inadequate communication among team members. In contrast, in this study in the SICU, inadequate communication included interactions with the patient, family, other healthcare team members, management, and physicians. Thus, this study extended the root causes identified by Hamric et al. (2012) by specifically identifying who the interactions were taking place between.

This method of qualitative descriptive research revealed root causes of moral distress, such as, clinical situations stemming from lack of resources and staff. Through the nurse participant's identification of a moral issue (i.e., unable to provide quality care the patient is paying for due to short-staffing on the SICU nightshift), in this particular case it was having a shortage of competent nurses to help with life-saving, trauma patient care. Thus, due to this lack of nurses, the underlying moral issue experienced by some 
nightshift nurse participants was the inability to provide quality care to all patients due to lack of staff. This lack of sufficient staffing led to some patients being ignored creating negative experiences and a sense of powerlessness for the nurse participants. This is similar to previous research that revealed the keen responsibility to patient care that is a part of the feeling of moral distress, in addition, concern for the patient's sense of wellbeing, but a concurrent and overwhelming perception of powerlessness to change the outcome (Dudzinski, 2016; Hamric et al., 2012). Some nurse participants described feeling powerless in this study in the SICU. Others compartmentalized their feelings. Furthermore, nurse participants noted frustration and emotion without not being able to "do their best" for the patient all the time.

Thus, the root causes of moral distress identified by this study are a result of clinical situations and have been primarily described by critical care nurses and include, but are not limited to, providing aggressive patient care in situations of futility—or a poor prognosis/predicted outcome, being a part of care that is not in the patient's best interests, and working with incompetent care providers (Hamric et al., 2012). Similarly, this study found that working with patients who have a poor prognosis and also incompetent care providers in the SICU influence how nurse participants perceive quality patient care and also their own ability to provide care that is in accordance with their personal values and duties. Specifically, nurse participants expressed frustration in working with new residents and having to inform the resident of the patient's condition, because of their lack of experience. The nurse participants expressed that this is the resident's responsibility. 
In addition, nurses in this study also noted that some physicians use their position of power to their advantage and coerce patients, families, and also nurses into agreeing with their pre-determined plan of care. Moreover, this study found that often interactions with families and other healthcare professionals, in particular physicians and new residents, impacted nurse participants' perceptions of the patient's outcome in cases where further treatment was deemed to be contributing to patient suffering. In contrast, this study also extended the consideration of futile and/or non-beneficial aggressive treatment near the end-of-life, particularly in cases where the outcome is uncertain. Nurse participants were less likely to quickly write-off a patient's potential recovery based on their experience in the SICU. Many nurse participants felt that even in these dire clinical situations where the outcome is uncertain, it is easy to give-up on the patient's recovery and only focus on how care is not beneficial.

However, nurse participants in this study, even those without a lot of SICU experience, still noted the importance of not giving up hope and maintaining positivity in the clinical. Thus, this is a new root cause of moral distress discovered by this studywhen nurses are unable to maintain positivity about the patient's outcome, then perhaps this leads to other members of the healthcare team "giving up" on the patient's potential for recovery. This was a new addition to the root causes of moral distress discovered by this study, because the source was not necessarily a negative experience, but a positive aspect of moral distress was identified by the nurse participants in doing their best everyday at work and also — not "writing off" a patient just based on a current poor prognosis. Thus, in contrast to Hamric et al.'s (2012) identification of various clinical situations that contribute to moral distress, it was discovered from this study that nurses 
maintain positivity and optimism, while also working to provide the best possible patient care. Alternatively, moral distress is a negative phenomenon as demonstrated by previous research (Epstein \& Hamric, 2009); however, this study demonstrated that despite this negative phenomenon—nurses maintain positivity and optimism about prognoses to keep moving forward and also work to provide the best possible care to patients that they treat like their own family members.

Root causes of moral distress that are a result of internal constraints include personal characteristics of the healthcare professional. Internal constraints of the healthcare professional include experiencing feelings such as perceived powerlessness or lack of knowledge of alternative treatment plans (Hamric et al., 2012). Thus, internal constraints are factors that result in root causes of moral distress that are inherent in the individual. Alternatively, this study found that internal constraints also include positive characteristics such as perseverance and empowerment, because of the recognized violation of one's moral values and duties. For example, this study found that nurse participants were already empowered, knowledgeable, and resourceful, because they were able to articulate why they felt morally distressed and specific coping methods they used to manage the moral residue. Therefore, internal constraints may also include personal characteristics beyond powerlessness and frustration, but also feelings of empowerment and perseverance for being able to recognize moral wrongs that occur at the bedside - even if they were unable to effectuate any change related to the particular clinical situation's outcome. Thus, moral residue ensued in these cases, but it did not stop nurse participants from working hard and providing the best possible care within their means. 
Finally, external constraints refer to the healthcare organization or system in which the nurse operates writ large (Hamric et al., 2012). Interestingly, this study supported the inclusion of the healthcare organizations as an external constraint. For example, within healthcare organizations or healthcare systems, power hierarchies exists, boards of administrative employees that make decisions about care provided at the bedside - in which they are far removed from practice, as well as institutional policies that conflict with care needs, such as compromising care due to pressures to reduce costs as just an example (Hamric et al., 2012). In contrast, external constraints identified in this study were more specific and focused on administration and management's decisions to delegate work—-such as extra checklists and also peer chart audits—-that bedside-level providers felt robbed them of the time necessary to provide their best quality care to the patient — which caused a significant amount of moral distress among nurse participants.

Nurse participants were able to describe numerous morally distressing clinical situations during all interviews that were specific to management. In particular, nurse participants noted lack of support from management and administration in staffing needs and also resources needed to provide quality patient care. Each of the 21 participants was able to recall at least one instance in their SICU experience that violated their core values and duties. The majority of nurse participants recalled numerous morally distressing clinical events—even nurses who had only worked for only a year or two described frequent occurrences. Thus, due to the volume of experiences, the themes and process of moral distress reveal that these nurse participants were experiencing the stain of moral residue. Therefore, moral distress may be understood as an acute reaction to a clinical situation that is perceived to be unethical. According to Epstein and Hamric (2009), 
moral residue is defined by the lingering feelings one experiences after a morally problematic clinical situation has passed.

Moral residue is the stain left on one's psyche from this initial encounter of moral distress. This study hypothesized that the more morally distressing clinical situations a nurse encounters, then it may be inferred that this nurse has unresolved moral distress in the staining form of moral residue. Interestingly, this study confirmed the presence of the moral residue - the nurse participants were not resolving their multiple experiences of moral distress that were accruing without incident.

According to Epstein and Hamric (2009), the crescendo effect model depicts how moral distress and moral residue, although conceptually different, are interrelated, because a buildup of moral residue appears to be dependent upon repeated experiences of moral distress. This study corroborated this finding, particularly when a nurse from one SICU described being burned out from working with other nurses who do not care for their patients to the best of their abilities and multiple end-of-life experiences where the nurse felt like she was torturing the patients. In addition, Epstein and Hamric (2009) noted that researchers of moral distress need to be alert to strong emotional reactions among nurses in difficult cases as this may indicate a violation of moral integrity, moral residue, and a developing crescendo effect. The nurse participant previously mentioned who was experiencing burnout displayed strong emotional and psychological distress about her experiences — which were morally distressing — and recalled specific times, clinical situations, dates, names, and events in great detail. The finding of alert and specific recall corroborates what Epstein and Hamric (2009) described as evidence for the presence of the crescendo effect. 
The crescendo effect was evident in the two nurse participants who could not take it anymore. Moreover, the piling up of unresolved morally distressing experiences was evidence of moral residue among the nurse participants. The crescendo effect was of interest in SICU nurses, particularly because previous research of SICU healthcare professionals (i.e., nurses, physicians, and other ancillary staff), when compared to MICU healthcare professionals, revealed that many SICU healthcare professionals did not report constructive coping behaviors (Bruce, Miller, \& Zimmerman, 2015). According to Bruce et al. (2015), these findings were perhaps attributed to the ICU unit culture and differences in professional backgrounds, rather than gender differences. It is unclear how Bruce et al. (2015) may completely discount gender differences, particularly since no male nurses were described in the three case studies selected in their study. In addition, this current study ensured that males were sampled in order to explore gender differences as a potential possibility. From this study, it remains unclear how moral residue and the eventual crescendo effect specifically develops for each individual, but it is clear that over time moral distress and moral residue result and are detrimental to SICU nurses' personal and professional roles.

It is still unclear whether unaddressed moral distress - or initial distress - is something that slowly accumulates over time as reactive distress or moral residue and then develops into a crescendo (e.g., similar to a linear stacking effect where the circumstances effectively compound on one another), or rather the sheer incredulousness and negativity from a single experience of moral distress by experienced or novice SICU nurses to recognize the futility of their ability to effectuate change for quality patient care. 
Interestingly, the results of this study revealed that all of the nurse participants perceived and experienced the signs and symptoms of moral distress. However, not every nurse participant progressed to the final stage of the process - moral crescendo. Of the 21 nurses interviewed for this study, only eight nurses (38\%) remain working at the bedside in SICU at this time. This represents a significant level of attrition and is not costeffective for a healthcare organization to experience this much attrition in an SICU environment. The healthcare organization has the responsibility to nursing staff to focus on the nurses' well-being and retention at the bedside through the provision of adequate resources. Nurse participants in this study noted that the administration and management "do not care" what they experience or "go through" as bedside nurses.

Interestingly, the nurse participants revealed that the SICU environment magnified their experience of stress. As a result, these nurses had to use selfperseveration to not only continue caring for their complex critically ill patients in the SICU, but also to protect their own well-being, because the organization did not provide adequate resources and advocate for nurses to stay at the bedside. Nurse participants candidly and genuinely displayed the inner-workings of their perspective. During the data gathering process of this study, issues in patient care were discussed that created emotions, stress, and memories in the nurse participants. Respect was maintained and shown in the set and setting in order to allow for a professional discussion.

A question left in this researcher's mind is at what point in each nurse participant's story is the process of moral distress more likely to develop moral residue that is damaging enough to eventually lead to a moral crescendo? Rather, how may healthcare organizations prevent this process from being generated by advocating for 
nurses' well-being the same way nurses care for patients? In particular, within the context of the SICU in a Level-1 trauma and teaching facility, it is important to prevent distressing events that lead to moral crescendo or intervene before the crescendo occurs, before the morally distressing experiences accumulate to the point that the stain of moral residue is indelible and inevitable, as exemplified by two nurses who were admitted for in-patient psychiatric treatment.

The perspective of each nurse participant's story is within a context of moral distress. This moral distress impacted the nurse participants as well as other individuals in the SICU environment. Importantly, each nurse participant had something positive to say about nursing. In a story of moral distress this positivity is important because it describes the perseverance inherent already within each SICU nurse. This was an unexpected finding because so many nurse participants were self-aware about why they liked nursing. Interestingly, "Doing your best" was identified as a central tenet of nursing care because it was achieved from a perspective of patient advocacy through caring, kindness, and compassion in each nurse participant's nursing care provided during every shift. Interestingly, not everyone progressed to a crescendo effect, but the name implies the building up of moral residue, although whether the development of moral residue occurs in a linear or circular manner remains unclear.

Clearly, all the SICU nurses identified a nursing care experience that was morally distressing. This event was either enough by itself or in context with other similar experiences to make these nurses question the ethics of their practice and their own personal views of right and wrong. But, the issue of nurses developing moral distress and everlasting moral residue moving on from the SICU environment highlights an 
individual-level problem among nurses, as well as a systemic- and unit-level problem in the healthcare organization and SICU, respectively. The purpose of this forthcoming discussion is to discuss the significance of each of the specific aims for this study - as they pertain to the identified themes, sub-themes, and process of moral distress, moral residue, and moral crescendo. Finally, the results of this study will be compared and contrasted with the current literature.

\section{Process of Moral Distress, Moral Residue, and the Crescendo Effect}

The process of moral distress was revealed by the nurse participants in this study, particularly how it applies to prior theories of moral crescendo, stress, and coping although this finding was unexpected. However, witnessing the process unfold in the data across the 21 SICU nurse participants was informative, descriptive, and humbling due to the nurse participants' vivid recollections, emotional processing that was still occurring from prior morally distressing patient care experiences, and the unexpected findings. Operating within this process are stress responses and subsequent coping methods used to manage morally distressing patient care situations that become psychologically and physically staining, creating moral residue. In this study, moral crescendo was evident in several nurse participants. To the best of this author's knowledge, there is a paucity of data in the nursing literature that specifically addresses perceptions and experiences related to moral crescendo.

Moreover, these experiences describe a rarely captured phenomenon pertaining to the identified process of moral distress, moral residue, and moral crescendo among bedside SICU nurses. Thus, these nurses' perceptions and experiences are invaluable for addressing system-level issues within the organization and creating policy change. 
According to Epstein and Hamric (2009), repeated experiences of moral distress are indicative of poor communication, inadequate collaboration, and perceived powerlessness from hierarchical organizational structures. Furthermore, Epstein and Hamric (2009) noted that the experience of moral distress among other healthcare professionals, not just nurses, supports an understanding of the systemic and ongoing nature of moral distress in healthcare organizations. Poignantly, Epstein and Hamric (2009) revealed that the crescendo effect is experienced and emanates from the structures where care is provided - healthcare organizations. Thus, this supports the need for a multifaceted approach to addressing moral distress and moral residue in the hospital: at the individual, unit, and organizational system-levels.

To the best of this author's knowledge, unique and novel to this study is the pursuit of this study's primary Aim 1: exploration of the process of moral distress, progressing to the unresolved stain of moral residue, and an eventual moral crescendo across 21 SICU nurse participant interviews. Furthermore, there is a dearth of studies that explicitly explore moral residue as a phenomenon (Epstein \& Hamric, 2009). Among nurse participants in this study, moral residue was present as evident in their vivid accounts of multiple patient care situations that violated their core values and duties as nurses - and continued to remain as unresolved feelings that were readily recalled.

Nurse participants in this study were hungry to talk about clinical situations that violated their core values and duties. Importantly, values, morality, and integrity were defined for the nurse participants in this study using lay terms. In fact, citing the common morality (Beauchamp \& Childress, 2009) refers to the norms of right and wrong in human conduct and are so universal that they naturally form a stable social compact. 
Nurse participants were able to understand a clear violation of their values and duties as professional nurses due to a perceived violation of their moral code in the clinical setting. This moral code violation is, in fact, manifested contextually around end-of-life issues, treatment courses, and the ethical dimensions of practice. Nurses are bound by a professional code of ethics to advocate for the safety and well-being of their patients. According to the ANA (2015) Code of Ethics for Nurses with Interpretive Statements, nurses are responsible for their moral environment and for respectful interactions, peer support, and identification of problems, which includes ethical investigation. Nurses, clinicians, educators, administrators, and researchers must consider the scope of moral distress through interventions that target individual, unit/team, and organization/system levels where moral distress occurs (Hamric, 2014). This comprehensive approach will provide nurses with the knowledge, tools, and resources to address moral distress.

Creating substantive change in practice requires acknowledging and addressing root causes of moral distress at an organizational and administrative level due to the pervasiveness of the phenomenon (Thomas \& McCullough, 2017). According to Sabin (2017), addressing the issue of moral distress should improve organizations. The American Association of Critical-Care Nurses (AACN) published a policy statement online that called for nursing programs to address and alleviate negative consequences of moral distress while also creating a healthy work environment (AACN, 2008). Creation of a healthy work environment is synonymous with an ethical workplace where nurses feel comfortable identifying and discussing ethical issues. Fostering an ethical climate/healthy workplace environment is consistent with the social determinants of health (Mason et al., 2016), the Triple Aim of a value-based healthcare system, the 
Affordable Care Act (ACA), the Institute of Medicine's (IOM) (2011) future of nursing goals, the ANA Code of Ethics for Nurses (2015), and the International Council of Nurses (ICN) (2011) Code of Ethics for Nurses. A revised combined policy statement of moral distress from professional organizations, such as the ANA and American Association of Critical Care Nurses (AACN), is necessary because influential bodies and professional organizations may influence administrative and legislative stakeholders to promote and implement comprehensive moral distress policy that impacts the organization's ethical work environments. Policy with influential support from credible organizations empowers hospitals to adopt change aimed at the individual/patient, unit/team, and institutional cultures.

Ethical issues and conversations are of paramount importance in questions of quality patient care, particularly as limited healthcare resources in hospitals and unlimited healthcare needs among patients converge on an already over-burdened nationwide healthcare system (Fleck, 2009). The need to communicate with nurses about ethical issues with respect to quality patient care was demonstrated by the results of this study. Interestingly, numerous nurse participants were moved to tears from recounting past morally distressing traumatic experiences, which had developed into a stain of moral residue due to the frequent number of patient care situations that were discussed at length. Thus, during the interviews nurse participants gushed forth with emotion, feelings, and vivid accounts, and would even apologize for their emotion.

The researcher reinforced the need to the nurse participants to not apologize for crying, but rather supporting their emotions which were indicative of their caring for the patient. According to Epstein et al. (2016), nursing ethics and moral distress consultants, 
relief was the most common response from healthcare professionals after they acknowledge their moral distress. For example, they noted in conversations with healthcare professionals that admitted moral distress were relieved that the powerlessness, responsibility, and guilt they felt about numerous patient care situations was not a sign of internal weakness or cowardice, but signs of caring, competence, and advocacy (Epstein et al., 2016).

Epstein and Hamric (2009) developed a theory of moral distress, moral residue, and the crescendo effect. This seminal article served as a keystone article for this study's literature review, because it posited the development of the crescendo effect, which describes the interrelationship between moral distress and moral residue (Epstein \& Hamric, 2009). According to Epstein and Hamric (2009), moral distress occurs when there is a perceived violation of a nurse's core moral values and professional duties, while also experiencing a feeling of being constrained from selecting the ethically appropriate course of action.

Nurse participants in this study confirmed the important designation of the troubling aspects of moral distress - the violation of a moral component. Nurse participants in this study experienced numerous morally distressing clinical situations that had progressed to moral residue, because their feelings were unresolved. At the core of all these situations was a moral issue. In particular, the SICU nurse participants' morally distressing experiences primarily related to end-of-life care and care of patients with poor prognoses that were receiving either too aggressive, or in some cases, too minimal care. Nurse participants' morally distressing experiences are also considered to be serious enough to be classified as moral residue, because they have progressed to the stage of 
being unresolved and in some cases manifest in professional changes in the nurse, such as burnout as identified in the case of one charge nurse participant. Furthermore, numerous personal changes were noted and may be classified as coping behaviors on a continuum of problem- and emotion-focused coping (Lazarus \& Folkman, 1984).

The antecedents and consequences of moral distress and moral residue in adult SICU nurses and the influence on perceived quality of care provided a clear view of the underlying process of moral distress, moral residue, and the crescendo effect. The identified themes, when taken together, illustrate a process of moral distress building into moral residue, and culminating in the climax of a moral crescendo. The first theme represents the process and development of an eventual crescendo. Theme 1 highlights the complexity of the SICU patients and the stress inherent in their care. In addition, Theme 2 highlights patient advocacy and positive nursing within a context of working around healthcare professionals who are perhaps higher in status, such as physicians. Unfortunately, some physicians leveraged this power differential to their advantage. Theme 3 highlighted the second part of the model for the process of moral distress, in which the SICU environment magnifies stress, thus the nurses have to focus on survival among this morally distressing environment of unethical problems. Importantly, as the themes of moral distress were revealed, it is necessary to recall the formula for the process identified in the data:

Nurses' patient care experience + SICU environment $=$ Moral distress (single event) $\rightarrow$

Moral residue (left unaddressed, accumulates) $\rightarrow$ Moral crescendo (quit, burnout, PTSD) 
For ease of interpretation, simple models of moral distress were developed to represent the complex, nuanced clinical situations in the SICU environment that led to negative experiences of moral distress for multiple nurse participants. Thus, bracketed within this equation above are the following assumptions to the proof:

\section{Complexity of SICU patients and stressful environment $=$ This SICU environment} creates stress for the nurse and the nurse has an initial response [positive, negative, or perhaps even neutral at the time that events are unfolding].

2. Complexity of providing nursing care for SICU patients $=$ STRESS accumulates over time as initial responses are not resolved, creating moral residue that stains the SICU nurses' experiences and perceptions.

3. Complexity of the SICU environment and inability to establish an outlet over time $=$ Moral Crescendo. It is noteworthy that this moral crescendo occurred only in a few cases in this study. For the most part the nurses were hopeful because they had other "outlets" for stress as they were in school and not "burned out". However, other SICU nurses were more isolated in their anxiety, except for the companionship of fellow coworkers who understood the moral distress of the SICU environment.

The goal of this research was to explore individual SICU nurse participants' perceptions and experiences with moral distress, moral residue, perceptions of quality patient care and stress and coping. Subsequently, these five themes illustrated the process of moral distress, moral residue, and moral crescendo. Conceptualized within each patient care scenario is a root cause of moral distress, which is subsequently connected, or rather part of a broader process of why that unethical act is being allowed to occur in a system. 
The process leads back to a system-level issue that if resolved, then more quality approaches to patient care may be achieved.

The interviews revealed five themes that illustrate the process about the development of moral distress and moral residue in the SICU setting: 1) some aspect of patient care creates a morally distressing scenario; 2) the SICU environment where the event takes place has numerous influences that are part of the process leading to the nurse's experience of moral distress; 3) moral distress is perceived and experienced through some specific clinical experience; 4) left unresolved, moral distress remains and seethes underneath the surface of the nurse's psyche through the stain of moral residue - meaning that the nurse may likely experience future, similarly morally distressing scenarios and recalls that there are still unanswered questions to arriving at a solution. Thus, they are prone to depression, anxiety, stress, drinking, drug use, exercise, and being emotionally upset about the way situations at work are handled; and 5) eventually a moral crescendo occurs where the clinical situation at that particular moment is too much or the bombardment of circumstances has resulted in a call to action on the part of the experiencing nurse. This final step is the coup de grâce that is the result of unaddressed moral distress. It is still unclear whether unaddressed moral distress, or initial distress, is something that slowly accumulates over time as reactive distress (or moral residue) and then develops into a crescendo (e.g., much like a linear stacking effect where the circumstances effectively compound on one another), or whether the sheer incredulousness and negativity from a single experience of moral distress is enough to let an experienced or novice nurse recognize the futility of their ability to effectuate change for the patient's quality care. 
Exploring the antecedents and consequences of moral distress and moral residue among SICU nurses and the influence on perceived quality of care is important because there may be a link between how SICU nurses feel over time with repeated exposures to morally distressing patient care situations and whether or not these experiences impact the nursing care they subsequently provide for complex and vulnerable SICU patients. According to Hiler (2018), in a study of critical care nurses, nurses who perceived positive quality of care actually reported lower levels of moral distress. Thus, it makes sense to foster open communication and transparency when addressing moral distress and moral residue at the bedside because it creates an environment to collaborate. This study identified that among SICU nurses, there is a connection between experiences of moral distress and the impact on perceived quality of care. For example, several nurse participants felt that even though they were troubled by past experiences of moral distress where they could not change the outcome for the patient, they felt that by concentrating on caring and "doing their best" they were able to manage the complexity of SICU patients and the stress involved. For example, Theme 1 - Concentrated Caring in High Stress Environment - "Doing Your Best" was identified because despite the complexity of SICU patients and the inherent stress involved with caring for them, SICU nurses maintained strength and resolve - and continued to work in the SICU, rather than leaving.

It is important to examine how the SICU nurses were able to stay working in the SICU environment. Specifically, the following sub-themes address this resolve, because even when involved in patient care scenarios where nurses could not completely provide the patient with the care they wanted, they still understood their professional roles and responsibilities. For example, the following sub-themes encompass Theme 1: "doing your 
best" for the patient, caring, communicating with families of patients near the end-of-life, and suffering and turmoil. Similarly, Epstein (2008) found, from her dissertation research regarding the lived experience of nurses and physicians after infant deaths in the neonatal ICU, that a main theme was being able to create the best possible experience for the family. This study in the SICU supported this finding by discovering that nurses just wanted to do their best. Whether it be their way of compartmentalization or coping, doing their best provided them with the impetus to keep caring at the bedside.

Interestingly, communicating with families was both a positive for some nurse participants, and created moral distress for others, because they did not feel supported by physicians, or administration and management with their ability to develop an ethical treatment plan. Interestingly, the CERN project found that if open communication was fostered during the acknowledgment of ethical issues then a positive impact was noted in improving the quality of patient care and mitigating nurses' experience of moral distress (Robinson et al., 2014). In this SICU study, often issues arose with families wanting to continue treatment despite the patient's poor prognosis. Also, there were instances where the healthcare team was continuing treatment and the nurses and family of the patient disagreed, thus moral distress ensued. Similarly, Piers et al. (2012) found among nursing homes and acute geriatric units that futile treatment at the end-of-life was associated with higher levels of moral distress. Furthermore, issues of futile treatment were considered inadequate if unjustifiable life support measures were used, unneeded procedures and tests, and incompetent healthcare professionals were working (Piers et al., 2012).

Shared understanding and mutual respect are necessary for integrity-preserving moral compromise, particularly during ethics consultations which organizations ought to 
promote for nurses, especially when they reach out and use these resources in order to encourage understanding and mutual respect in the resolution of ethical issues (Webster \& Baylis, 2000). According to Reddy et al. (2015), patient and family input are vital in determining goals of care at the end-of-life. Situations of futile treatment may be avoided if patient and family goals are acknowledged, discussed, and provided consideration throughout goals of care discussion - the goal being to attenuate instances of futile treatment (Reddy et al., 2015). Moreover, some physicians impeded the process due to multiple teams presenting families with disparate sources of information that made determining treatment goals at the end-of-life difficult. Furthermore, as a result of this lack of consensus, SICU nurse participants felt that the patients were undergoing unnecessary suffering and in turmoil about these conditions that were allowed - and even enabled - to persist.

Interestingly, Mobley et al. (2007) discovered that moral distress in critical care nurses related to futile treatment has been related to nurses greater than 33 years of age, having more than four years of experience in the ICU, and having more than four years of nursing experience. Thus, the perception of futile treatment is higher the more experience the nurse has; however, this study in the SICU demonstrated that young and experienced nurses both exhibited signs of moral distress related to end-of-life care. Furthermore, nurses do not have a clearly defined role as far as their participation in decision-making at the end-of-life, particularly during end-of-life family meetings were goals of care are discussed (Flannery et al., 2016; Hamric et al., 2012; Robinson, 2010). However, since nurses have more contact with the patient than any other healthcare professional, it only makes sense to include their participation, but it also is understandable why nurses may 
experience moral distress because of their lack of involvement in these discussions, but concurrent involvement in every aspect of the patient's care (Flannery et al., 2016; Hamric et al., 2012; Robinson, 2010).

\section{Coping with the Stress of Moral Distress and Moral Residue}

Building upon Epstein's dissertation research (Epstein, 2007), Epstein and Hamric (2009) hypothesized that moral residue may eventually crescendo and become unmanageable and may manifest professionally as conscientious objection, emotional and psychological distancing from the ethical dimensions of practice, burnout, and/or withdrawal from the nursing profession. Similarly, a study on life-sustaining treatments found that nurses and physicians felt that they acted against their conscience when providing care to dying patients (Solomon et al., 1993). According to Vacoe et al. (2012), the moral distress that some nurses experience may be a signal for the turnover, burnout, and resignation experienced on a nursing unit. Interestingly, Vahey et al. (2014) revealed that if patients were cared for on units where nurses are staffed appropriately, supported by management, and have friendly interactions with physicians and other nurses, then the patients are more likely to be satisfied with their care. Furthermore, Vahey et al. (2014) noted that nurses noted lower levels of burnout as a result and thus levels of nursing burnout were impacted by patient satisfaction. This evidence demonstrates the close relationship between patient care and nursing satisfaction, particularly as it relates to nurses actually being able to do their job effectively with enough resources. This SICU study supported these results because nurse participants felt that they were able to do their job to the best of their ability when they worked with a great group of nurses that 
facilitated teamwork. Furthermore, when the SICU was staffed appropriately, the nurse participants felt that they were able to meet all their patient and family care needs.

It remains unclear how the crescendo effect evolves over time and with the intensity and frequency of morally distressing root causes. This study was not able to determine a time frame, because nurses were not interviewed when the situation occurred. Rather, the majority of nurse participants were recounting past experiences. Thus, based on the results of this study, it is clear that the crescendo effect does occur. Nurses may cope with moral distress by using problem- and emotion-focused coping (Lazarus \& Folkman, 1984).

In this study it was found that nurse participants practiced on a continuum of problem- and emotion-focused coping with respect to moral distress. Because no two people cope with distress in the same way, it was important to explore SICU nurses' coping abilities with moral distress because there are constructive (e.g., journaling, exercise, communicating with a work peer) and maladaptive (e.g., excessive alcohol consumption, smoking cigarettes, illicit drug usage) coping behaviors to reconcile nurses' experiences of powerlessness associated with moral distress.

Additionally, nurse participants' problem- and emotion-focused coping with moral distress and moral residue was explored. For example, nurse participants were asked to describe their professional and personal coping methods and behaviors related to moral distress and subsequent moral residue in the SICU to determine if they are temporally, contextually, and/or situationally-specific to particular root causes of moral distress. According to previous research, the keen responsibility of the moral distress experience depends on temporal, contextual, and situation-specific factors (Dudzinski, 
2016; Epstein \& Hamric, 2009; Hamric, 2014; Hamric et al., 2012). For example, according to Webster and Baylis (2000), when nurses adapt their values and change their duties based on repeated experiences of moral distress then this results in desensitization to wrongdoing, which in a healthcare context-where the margin for error is almost nonexistent—represents a lack of quality patient care. This study revealed that context is perhaps the most important factor due to the sheer detail that each nurse participant was able to provide. Interestingly, it is still unknown about the effect of the timing of morally distressing events, primarily because this study was not able to verify empirically at what point in the nurse participants' SICU tenure did the most morally distressing event occur and when the subsequent coping occurred as these initial distresses stained with moral residue. To the best of this author's knowledge, this study is unique in that it captured all three concepts - moral distress, moral residue, and the crescendo effect in the adult nurse SICU context.

Nurse participants' professional and personal coping methods and behaviors related to moral distress and subsequent moral residue in the SICU were explored. However, it still remains difficult to determine how the frequency and intensity of the morally distressing event impacts the SICU nurses' experience. According to JohnsonCoyle et al. (2016), nurses and nurse practitioners with more experience in the cardiovascular surgical ICU had greater levels of moral distress. Furthermore, these nurses and nurse practitioners also felt that the level of disturbance, or intensity, impacted their perceptions of moral distress, more than the frequency of the events occurring (Johnson-Coyle et al., 2016). In addition, Dekeyser and Berkovitz (2012), round that lower levels of quality nursing care, nursing skill, and being able to meet patient's needs 
was related to higher frequencies of moral distress, but interestingly, these feelings were not found to impact quality of care. Similarly, Hiler (2018), found that the intensity of moral distress does not relate to perceptions of quality of care-only the frequency of occurrences. Thus, the academic literature requires clarification. This study noted that nurse participants with less than a year of experience were morally distressed. Thus, it is a combination of the frequency of occurrences and the absolute intensity of each of those experiences as they are disturbing. This study was not able to empirically demonstrate which aspect of moral distress was more troubling - the intensity or the frequency, only that it was a pervasive problem across the board for all SICU nurses and was causing them to seek other jobs and go back to school.

For example, it is clear that the majority of nurses were experiencing moral residue due to the multiple, frequent, traumatic vivid clinical situation examples they were able to acknowledge. Moreover, it is clear, from the two nurse participants who experienced the crescendo effect, that prior experiences of moral distress that have stained into the taint of moral residue are also indicative of future experiences of moral distress. Meaning that if a nurse has experienced moral distress in the SICU environment in the past, then morally distressing patient care situations will likely occur in the future-provided the context in a Level-1 SICU. Thus, coping becomes an important focus as SICU nurses remain at the bedside. According to Henrich et al. (2017), nurses managed the negativity of moral distress with several coping techniques that included venting, debriefing, and discussing the experiences with colleagues. Importantly, Henrich et al. (2017) discovered that some healthcare professionals coped with their moral distress by becoming less invested in their work environment, thus they were able to 
distance themselves from their morally distressing experiences. This study in the SICU demonstrated that this a marker of moral residue, and a precursor to the crescendo effect. These nurses have to cope in order to come back to work the next shift and do it all over again.

Nurse participants in this study were asked about their individual coping. Most often nurse participants would hangout and communicate with co-workers from the SICU, because nurse participants felt comfortable sharing sensitive and stressful information with these co-workers. The nurse participants said that nurses they worked with on the SICU were the only ones who understood what they were going througheven more so than significant others at home, who did not work in healthcare.

Interestingly, Theme 4 identified by all of the SICU nurse participants, ways of individual coping with the stress of the stain of moral residue. Importantly, the psychological stress these nurse participants were experiencing was a result of the violation to their professional core values and duties. Thus, they had to cope to survive in order to deal with the impact on their own personal moral integrity and values. For example, this study explored nurse participants' individual styles of coping and ways of surviving to violation of their core moral integrity, values, and duties as a healthcare provider. Furthermore, no judgment was made regarding the nurse participants responses, thus, no method was undertaken to classify particular coping styles and maladaptive or constructive, as Bruce et al. (2015) parsed out in their study. Instead, the focus of this study was to explore how nurses individually coped with the moral distress and moral residue of their clinical work environment in the SICU. Importantly, the majority of SICU nurses compartmentalized in order to cope. 
Previous research on morally distressing experiences is common and compares appropriately with this study. First, it must be communicated that Choe et al. (2015) did not distinguish critical care providers by specialty ICU such as medical versus surgical ICU. Alternatively, according to Bruce et al. (2015), many healthcare professions in the SICU did not report constructive coping mechanisms to moral distress. Additionally, Bruce et al. (2015) noted that research has yet to document maladaptive responses to moral distress and how they evolve (Bruce et al., 2015). Thus, this SICU study solely focused on the SICU environment. Interestingly, Choe et al.'s (2015) phenomenological study that explored critical care nurses experience of moral distress did not distinguish among ICU specialties. Thus, this may be a limitation as interventions are sought to improve empowerment and actionable change towards addressing moral distress in the SICU environment. For example, fourteen critical care nurses were interviewed in-depth, which is beneficial and informative as noted in this study. According to Choe et al. (2015), short staffing is compounded by increased turnover related to ethical suffering. Moreover, Choe et al. (2015) revealed that this shortage of staffing may subsequently lead to added moral conflicts as part of a vicious cycle that also decreases patient satisfaction.

In this study, in-depth interviews with 21 SICU nurses provided rich, descriptive accounts of moral distress and highlighted the context in which those patient care scenarios "played out" - in the SICU, which Choe et al.'s (2015) study did not distinguish between particular ICUs. This lack of distinction is important because Bruce et al. (2015) noted that many healthcare professions in the SICU did not report constructive coping mechanisms to moral distress. In fact, nurses in the SICU 
demonstrated more maladaptive coping mechanisms. Thus, this is an important environment to study given the traumatic clinical situations that inundate this context. However, Choe et al.'s (2015) qualitative exploration of critical care nurses' moral distress is important because even though it did not differentiate between ICU specialties, participants experienced burnout, high turnover, and decreased quality of care. Importantly, these findings occur widely in ICU nurses.

Thus, the SICU environment was an important sample to study and parse out from other ICUs and the critical care setting in general, due to the lack of constructive coping as previous research demonstrated (Bruce et al., 2015) and from previous research that demonstrated critical care nurses in general experience high burnout, high turnover, and decreased quality of care (Choe et al., 2015). However, this study explicitly focused on SICU nurses' moral distress and coping extending a study by Bruce et al. (2015) who found that SICU healthcare professionals, unlike medical ICU healthcare professionals, were less likely to report constructive coping behaviors like venting, mentoring, and building team cohesion. Similarly, nurse participants in this study noted that their quality of care diminished as unresolved, morally distressing events developed into moral residue, due to lack of time to process emotionally and cognitively the numerous traumatic clinical situations they encountered on a daily basis.

Compartmentalization for these nurses included drinking, eating, and sleeping, but for others compartmentalization meant not ruminating on courses of action they could not change. For example, even though aggressive treatment at the end-of-life in a patient with a poor prognosis was morally distressing at the time, in order to come back to work and continue working, these nurse participants found the importance of distraction and 
healthy behaviors such as reading, exercise, communicating about their distress, spending time with family, cooking, and/or just trying not to solve every problem on a moral level, but trying to deal with events as they occur- due to the fast-paced nature of the Level-I SICU environment.

Nurse participants in Lievrouw et al.'s (2016) study had a tendency to either internalize or externalize their experiences of moral distress and focused on rational or experiential moral distress elements. This prior research informed this study as similar coping characteristics were described by adult SICU nurses, such as being thorough, autonomous, compromising, and intuitive. Alternatively, Lievrouw et al.'s (2016) focus was on individual coping styles, but the authors noted that moral distress seems linked to broader organizational contexts, which this study corroborated as well as others in their work about moral distress being conveyed as an inherent systems-level problem that also includes units/teams, and individuals (Epstein \& Hamric, 2009; Hamric, 2014; Hamric et al., 2012).

The scope of moral distress is vast and wide in healthcare. However, it remained important in the design of this qualitative descriptive study to be reasonable and wellconsidered in one's sampling, data collection, data analysis, and re-presentation techniques in order to obtain from the SICU nurse participants straight and mostly unadorned (i.e., minimal theorization, transformation, or spin — thus, theoretical sensitivity) answers to questions of special relevance to moral distress, moral residue, stress, and coping in the SICU environment-in particular, of importance to healthcare practitioners and policy makers, which is why addressing and acknowledging moral distress and moral residue is so vitally important (Sandelowski, 2000; Sandelowski, 
2010). The goal of this study was not to lead nurse participants to answers, but rather let the data speak for itself and convey meaning through the nurse participants' perceptions and experiences.

Lievrouw et al. (2016) created a theoretical model of moral distress coping strategies based on the results of their study. According to Lievrouw et al. (2016), their theoretical model posited visually on various axes, for example, the four dominant ways of coping with moral distress: a) thoroughness (let's do it right); b) autonomy (let's do it now); c) compromise (let's do it harmoniously); and d) intuition (let's do it intuitively). The horizontal $\mathrm{x}$-axis depicted a continuum of coping strategies from a tendency to internalize towards the left, to a tendency to externalize as the axis moves towards the right. The vertical y-axis also represents a continuum of coping strategies with a tendency to focus on rational elements at the top and towards the bottom of the axis is a tendency to focus on experiential elements. Thus, the researchers categorized participants' styles of coping along the theorized model. Specifically, Lievrouw et al. (2016) found that physicians primarily reported rational ways of coping with moral distress; however, nurses tended to focus on feelings and experiences. Interesting, in Lievrouw et al.'s (2016) study, nurses and new physicians did not always feel confident asserting moral beliefs about the immorality of patient care. Thus, these researchers argued that the nurses and new physicians chose a more rational way of coping by granting autonomy to their superiors, while never actually using their preferred coping style because they turfed the issue to their superior. Arguably, Lievrouw et al. (2016) stated that these participants may not even use their preferred style of coping until later in their career when they gain more experience and comfort with doing so, or perhaps work in a different setting, 
resulting in different support preferences. Lievrouw et al. (2016) focused on the coping methods in gastroenterology and medical oncology. In addition, Lievrouw et al. (2016) included physicians and nurses. In comparison, this study interviewed 21 nurse participants and focused entirely on the SICU environment. Thus, the goal was not to create a theory or even uncover a process of moral distress, moral residue, and the crescendo effect. Instead, the goal was to explore SICU nurses' perceptions and experiences with care that violated their professional values and duties as a nurse. Not only did individual coping methods of each individual SICU nurse receive attention, but also how they compartmentalized their distressing experiences. Unlike, Lievrouw et al.'s (2016) documentation of various coping styles, this particular study aimed to understand how nurses survived and compartmentalized the moral atrocities that occurred daily in practice in the SICU — and what kept them coming back. Thus, this study focused on the individual SICU nurses and each of their stories and how they fit with the broader context and uncovered process of moral distress, moral residue, and the crescendo effect.

The goal of this study was to explore the perceptions and experiences with moral distress, moral residue, perceptions of quality patient care, and stress and coping of adult SICU nurses. This study used research to construct a theoretical model of moral distress, stress and coping, moral residue, and perceptions of quality patient care that was developed prior to conducting the study. However, the study's results illustrated the themes and uncovered the process of moral distress, moral residue, and the crescendo effect based upon nurse participants' own words.

This theoretical model is not born out of the results of the study similar to Lievrouw et al.'s (2016) study. Rather, the model was created to explain a phenomenon 
that already has been documented in the scientific literature - moral distress and moral residue. Instead, the themes of these interviews uncovered an unexpected finding - the process of moral distress, moral residue, and the crescendo effect. The underlying process of moral distress, moral residue, and moral crescendo confirms the model that Epstein and Hamric (2009) created, but also extends the knowledge by uncovering that many smart, caring, and effective SICU nurses are currently managing numerous, traumatic stains of moral residue. Uncovering this finding is necessary to acknowledging and addressing the SICU nurses' moral distress.

Each nurse reacts differently, thus exploring the phenomenon across multiple SICUs was warranted. Unlike previous research, this study found that nurses who experienced moral distress, moral residue, and the crescendo effect were not overly emotional or merely caught-up in their feelings. Instead, this study found that nurses were rational, intelligent, competent, caring, and intentional in their practice. To say that physicians respond more rationally than nurses is inaccurate and insulting to their nursing practice. According to Lievrouw et al. (2016), physicians preferred a rational coping style, while nurses focused on their emotions and feelings (Lievrouw et al., 2016). This is consistent with Bruce et al. (2015) identifying that physicians focused on science, while nurses focused on humanistic elements. In contrast, this study's results diverge from these findings, because it was found that nurses, while they do focus on feelings, emotions, and the holistic aspects of care - they are also rational, competent, and educated in evidence-based research and practice. Thus, it seems counter-productive to dichotomize coping as previous research has suggested. 
From this study, the task of categorizing coping methods was difficult. For example, to say whether or not particular methods of coping are problem- or emotionfocused coping requires that context about the clinical situation they are describing be known. Additionally, some familiarity with the individual is required in order to assess and evaluate how they are responding in the described scenario versus baseline interactions - where moral violations to core values and duties are absent. For example, if nurses become emotionally invested, then it this is classified as emotion-focused coping. However, if this type of coping is done in order to maintain a human connection to the patient and thus provide care that is moral, then in accordance with Lazarus and Folkman (1984), it may be difficult to tease out which is problem- and emotion-focused. For example, a nurse experiencing moral distress became more emotionally invested in a particular clinical situation, but if this was done in order to provide care that is compassionate, then the nurse has used this integration of emotion into care to solve the problem of assisting the patient respond to treatments-which was more of the rational and objective part of focused-coping — or rather problem/goal-oriented focus. Thus, the emotion-focused coping may be a way of solving problems more effectively and thus it is difficult to tease out the two types of coping as Lazarus and Folkman (1984) noted regarding a blending of the two styles in moments such as warfare or high stress. Lievrouw et al. (2016) noted that physicians and nurses changed their ways of managing moral distress depending on personal or work-related experiences, and the perceived team culture.

This study explored adult SICU nurses and found that nurses who had more SICU experience were not more likely to notice violations to their core values and duties, 
because both experienced and inexperienced nurse participants were able to recognize these violations during interviews. Rather, experienced nurses seemed to compartmentalize the morally distressing aspects of their job more efficiently, thus the experienced nurses had appreciable stains of moral residue. They were able to recount particular events, names, dates, and did so rationally and competently—while also being emotional and feeling for the patient's well-being. However, when these nurse participants opened up about their perceptions and experiences, their responses were similar to less experienced nurses, however, they simply had a better handle on compartmentalizing and moving forward each day. These results do not discount that some other ways of managing moral distress may not be dependent on the profession, but also the type of clinical situation and the context in which it occurs. Alternatively, Johnson-Coyle et al. (2016) suggested that cardiovascular surgical ICU nurses with more experience exhibited higher levels of moral distress. Furthermore, these more experienced nurses' perceptions of moral distress were impacted by the level of disturbance, rather than how many of the clinical situations actually occurred (JohnsonCoyle et al., 2016). Thus, previous research suggests that experience and type of disturbance plays a role. However, this study did not distinguish whether or not the type of clinical situation versus the frequency of said situation impacted moral distress perceptions differently. Instead, this study explored the violation of core duties and values as a necessary characteristic of moral distress, which subsequently evolved into the unresolved emotions and feelings that occur via the compounding impact of moral residue. 
This study focused on coping in order to identify how SICU nurse participants were managing their perceptions and experiences of immorality while providing care at the bedside. According to Bruce et al. (2015), their study revealed key sources of moral distress among diverse ICU team members and how this moral distress is managed in the current team-based model. Moreover, Bruce et al. (2015) found that unit-based and intrateam dynamics play a significant role in moral distress. For example, Bruce et al. (2015) determined that if physicians become more detached as a complex patient care case progresses and the nurses become emotionally invested, then the healthcare professionals may not be able to communicate effectively and make appropriate decisions, because they are not seeking the same goals. Interestingly, this study found that a part of team dynamics that is most frustrating for SICU nurses is communicating with families, patients, and other healthcare team members, particularly physicians. The issues discussed from these interviews revealed that nurses get emotionally invested in their patients, but they are just as likely to be rational, objective, and even in some cases aloof. Thus, it is a mischaracterization and counterproductive to dichotomize physicians and nurses in the literature. According to Bruce et al.'s (2015) study, constructive moral distress coping behaviors were more prominent in the medical ICU, although many healthcare professionals in the SICU did not report constructive behaviors. In contrast, this study uncovered part of the reason why the lack of reported constructive behaviors was reported among SICU nurses - the uncovered and damaging process of moral distress, moral residue, and the crescendo effect.

The results of this study indicated that moral distress is a result of the high-stress environment in which SICU nurses practice. For example, Bruce et al. (2015) found that 
one of the greatest contributors of moral distress was intra-team dynamics. Alternatively, the greatest contributors of moral distress in this study centered around end-of-life issues. Although nurse participants in this study did confirm that communicating with physicians was morally distressing, but not because it was difficult to communicate among the different teams as in Bruce et al.’s (2015).

Rather, the nurse participants in this study found that physicians used their power as leverage and made the nurse feel incompetent. Additionally, physicians were often incompetent and did not communicate their intentions (e.g., such as making ventilator changes and not communicating the changes to the respiratory therapist or nurse in charge of the patient), or the physicians were not up-front and honest with the family about the goals of care and used this to pursue a goals of care agenda that was separate from what the family desired. This latter result of the study corroborates Bruce et al.'s (2015) study where clinicians were not forthcoming with patients and families when there were disparate clinical opinions. Interestingly, this study also found that multiple specialty teams of physicians caring for a trauma patient was difficult, and at times morally distressing, for nurses because they were unable to focus on one overarching plan of care to communicate with the family and often they felt that the physicians were coercing families into making quick decisions.

In this study, the underlying process of moral distress, moral residue, and moral crescendo was revealed among SICU nurses, because of the SICU environment, the complexity of patients treated, the ensuing stress associated with end-of life decisions, and the lack of support and resources from management and administration. Thus, the perfect storm exists where adaptive coping behaviors must be used in order for the SICU 
nurses to survive in the SICU environment. This corroborates prior research and theoretical work by Epstein and Hamric (2009) through their moral crescendo theory. For example, through repeated experiences of moral distress, and subsequent compiling of moral residue, the process of moral distress evolves over time leading to a crescendo that is experienced by the SICU nurses as they cope with the immorality of patient care that becomes so overwhelming that it is difficult for the nurses to function as healthcare providers.

Bruce et al.’s (2015) research demonstrated that SICU healthcare professionals employed fewer constructive coping methods, than medical ICU healthcare professionals. For example, SICU healthcare professionals were more likely to report maladaptive coping behaviors such as pas-de-deux, fighting, and desensitization, while MICU healthcare professionals were more likely to report constructive coping behaviors with managing their sources of moral distress such as venting, mentoring, and building team cohesion (Bruce et al., 2015). According to Bruce et al. (2015), maladaptive behaviors used to manage moral distress by medical and surgical ICU nurses, physicians, and other ancillary staff include: (a) pas-de-deux - which involved communication regarding a complex navigation and resolution of multiple sources of information from multiple healthcare team members in order to resolve multiple opinions (e.g., it is considered negative because it resolves opinions, while also incorporating all perspectives at the same time, which does nothing to effectuate any understanding of the clinical situation); (b) communication that focused on superficial conversations with family and communication that used conditional statements like "we're not sure" with family members in goals of care conversations; (c) fighting with other healthcare team members 
through confrontation; and (d) desensitization - when the healthcare professionals withdraw or detach completely from practice because of repeatedly feeling like they are unable to protect their professional integrity.

Interestingly, in this study, many nurse participants discussed ways of building team cohesion among other SICU nurses such as holding food pitch-ins and helping each other through teamwork and communicating or socializing with each other and going-out for drinks. SICU nurse participants in this study did not mention fighting. However, they did feel desensitized similar to colleagues in Bruce et al.'s (2015) study, because nurse participants felt that they did not currently provide patient care like they did when they first started — which many nurse participants who were experiencing moral residue also reported, resonating with the sentiment of becoming jaded.

The SICU environment was the focus of the study because it was unclear if the culture created an environment in which the lack of constructive coping persisted as a result, or the background of the SICU nurses, the nature and type of complex patients they treat, or some other unknown reason influenced their coping ability. Interestingly, it was determined that the underlying process of moral distress, moral residue, and moral crescendo is a combination of the SICU environment, the types of complex patients, the subsequent stress that ensues, and the lack of support and resources from management and administration. In contrast, Bruce et al. (2015) did not explore moral residue and how the stain of the residue influences the experience of moral distress over time, which this study rectified by exploring through individual interviews of SICU nurses working in two different SICUs in one trauma facility. For example, because the nurse participants were emotional in their accounts and subsequently recalled specific facts, times, and dates of 
multiple morally distressing clinical situations as they unfolded, SICU nurses provided direct evidence of moral residue. According to Bruce et al. (2015), maladaptive coping with moral distress included pas-de-deux - fighting and withdrawing - while constructive coping included methods such as venting, mentoring networks, and building team cohesion.

Similar to Bruce et al.'s (2015) study, SICU nurse participants in this study identified numerous approaches to decrease moral distress before becoming withdrawn from the negative ethical dimensions of their practice. For example, nurse participants in this study compartmentalized and survived. Although some nurses participants were angered by other nurses who seem to "just punch the clock". Thus, the nurse participants in this study were morally distressed, and stained with moral residue, but they still became flustered when they encountered co-workers not doing their best for the patient. Interestingly, some maladaptive coping discovered in this study included drug usage, alcohol consumption, consuming tobacco products, eating to excess, sleeping to excess, and also simply "signing-off" and choosing not to deal with the traumatic events they were facing everyday. Thus, the nurse participants were responding to the environment by coping and surviving. Still other nurse participants focused on exercise, increasing their knowledge by either reading or going back to graduate school, communicating about their distress to friends and family, or simply talking about the issues with a trusted SICU nurse co-worker.

Moreover, Lievrouw et al. (2016) studied coping with moral distress in the oncology setting and found that four dominant ways of coping emerged: thoroughness, autonomy, compromised, and intuition. Their findings revealed that physicians and 
nurses had a tendency to internalize or externalize moral distress and had a propensity to focus on rational or experiential elements (Lievrouw et al., 2016). Interestingly, this study found that nurse participants also internalize or externalize their moral distress.

It is easy to say one internalizes or one externalizes their thoughts and emotions. However, the nurse participants in this study seemed to be fighting two battles: a) working in a traumatic SICU hospital environment where cognitive and emotional processing about the care provided is not acknowledged, addressed, or encouraged; and b) going home and maintaining a quality of life separate from work. While coping may be different across different hospital settings and units, it is important to identify particular coping habits among nurses and other healthcare professionals who deal with moral issues on a daily basis in their work environments. Nurse participants in this study worked hard in the SICU. Nurse participants in this study were hard on themselves due to explaining that their behavior is a result of "just the job". However, part of the interview process was to uncover that the nurse participants' coping behaviors was a result of violations to their core values and duties as nurses providing care. Thus, it is easier to view coping in this context as less of a personal failure and more a response to ethical wrongs perpetuating from a healthcare system.

Other coping strategies Lievrouw et al. (2016) revealed included: changing a patient assignment, taking a break from the ICU and working in another area, journaling, and drinking alcohol after work. Nurses in this study would communicate with charge nurses in order to arrive at a patient assignment that they felt was safe and achievable for 12-hour shift. Furthermore, some nurse participants in this study confronted charge nurses with issues that were resolved through a patient assignment change. Thus, the 
methods of coping in this study were consistent with previous literature. Moreover, many nurse participants described using substances to relax and forget the stress of work.

Although a full-time schedule of three, twelve hour shifts a week seemed appealing to the nurse participants; however, they complained that it was too difficult to pick up extra shifts to make money, because of the toll it took on them. It is important to understand the symptoms of moral distress because these symptoms provide insight into how damaging the phenomenon is to SICU nurses who experience its negative effects. In this study, SICU nurse participants named coping strategies in the SICU environment that coincided with communication. For example, nurse participants in this study enjoyed communicating with friends, family, and significant others about work initially when they first started the job. However, as time progressed, they found more benefit and satisfaction in going out with friends from the SICU, partying with friends from the SICU, and discussing difficult clinical situations with their fellow peers from the SICU. The nurse participants cited understanding and unity among colleagues as the major reason for experiencing positivity and being able to carry-on everyday with efforts to do their best, and the "right thing" regarding patient care. Thus, a major way to cope with moral distress in the professional SICU environment is through intentional communication that is deliberate and transparent.

Nurse participants in this study communicated in a variety of ways. For example, nurse participants communicated with each other frequently and found camaraderie in communication, because they were able to work through morally distressing clinical situations together. Furthermore, important to this process was debriefing. For example, nurse participants discussed going to grab a beer with a co-worker after a particularly 
traumatic day at work. This is a form of therapy and de-briefing that needs to be honed in on, because it is a valuable method of therapeutic communication for these nurses discovered in this study. Alternatively, nurses also found communication morally distressing, such as when physicians used their power differential and status as leverage in discussions with nurses and families. Thus, communication could be therapeutic among other nurses, but morally distressing across disciplines, which corroborates intrateam dynamics leading to moral distress as Lievrouw et al. (2016) contended.

This study explored SICU nurses' perceptions and coping abilities in the midst of moral distress, moral residue, and moral crescendo in the SICU environment. Interestingly, Henrich et al. (2017) found from 10 focus groups with 56 participants (nurses and healthcare professionals) in 13 ICUs in the Vancouver, British Columbia, Canada, that coping with emotions through venting, debriefing, and discussing the morally distressing situation with compassionate colleagues were successful methods to manage the negative emotional responses to moral distress. Furthermore, Henrich et al. (2017) revealed that nurses and other healthcare providers frequently maintained that it was best to address the cause of the morally distressing critical event. Similarly, this is critical information that this study addressed because identifying the original root cause of moral distress was the moral issue that precipitated the morally distressing situation and why that particular instance was morally wrong.

According to Henrich et al. (2017), focus groups revealed that staff who experience moral distress primarily feel frustrated. Furthermore, Henrich et al. (2017) revealed that healthcare professionals in their study coped with their moral distress by talking to supportive colleagues who provide emotional support, and gain an alleviation 
of their emotional turmoil from venting. Interestingly, this study confirmed these results because nurse participants gained a tremendous amount of therapeutic relief from communicating with supportive fellow SICU nurses. Similarly, nurse participants were frustrated in this study. However, nurse participants' frustration was extended into a deep-seated trauma and emotion that manifested as a stain of moral residue among all of these vibrant nurse participants. For example, it was evident that these SICU nurses were repressing unprocessed feelings, emotions, and severe violations to their core values and duties as a nurse.

An unexpected finding of this study is the amount of positivity that the SICU nurse participants described with respect to nursing to maintain optimism and cope with the severity of complex conditions and morally distressing situations they encountered at work in the SICU environment. For example, similar to Henrich et al.'s (2017), nurse participants in this study were able to identify the cause of the morally distressing event and felt that it was best to address that root cause. Yet in this study, SICU nurse participants tried to address the cause but were unsuccessful because there was a lack of support and resources from administration and management. In contrast, Lievrouw's et al. (2016) study primarily assessed nurses' coping in an oncology setting, which encompasses a different environment. The SICU environment is a key factor in the SICU nurse participants' experiences of moral distress and moral residue due to the complexity of the patient population with traumatic and acute injuries. Furthermore, this SICU environment creates a context where families must decide whether or not to treat loved ones who have had numerous acute traumatic injuries due to accidents that often involve young otherwise healthy people, rather than chronic conditions. Thus, the uniqueness of 
this current study is the focus on SICU nurses providing care for patients in a Level-I trauma SICU environment. Thus, this Level-I trauma environment provides a unique insight into the coping and behavior of SICU nurses, particularly as they try to provide the best possible quality and ethical patient care as they face multiple morally distressing situations.

Interestingly, this study explicitly explored moral distress and moral residue among nurse participants as it was classified according to their survival and coping with the repeated violation of their core values and duties. For example, participants described feeling stressed at work and anxious about morally distressing events, or rather events that would occur out of their control and they would subsequently feel horrible and carry enormous amounts of guilt, because they felt moral wrongs occurred as a result of violations to their values and duties as a nurse. According to Epstein and Hamric (2009), moral residue is difficult to characterize because it does not occur in consistent patterns or time frames and has also not been studied explicitly. Epstein and Hamric (2009) reported that personal (e.g., anxiety, depression) and professional changes (e.g., avoiding particular patients, burnout) are consistent with a loss of one's moral integrity, which is evidence for the stain of moral residue. This study identified and corroborated these findings. In addition, this study demonstrated the completion of the process, which was the unexpected and novel finding of this study - illustration of the process of moral distress, developing into unresolved feelings and perceptions through moral residue, and final completion through two individuals experiencing a crescendo effect.

\section{Exploring Moral Distress, Moral Residue, and the Crescendo Effect}


The purpose of this study was to explore the perceptions and experiences of moral distress and moral residue in adult SICU nurses. Exploring the perceptions and experiences of moral distress and moral residue in adult SICU nurses is clinically significant, because the negative consequences affect SICU nurses' ability to practice due to damage to their personal moral integrity and values. Nurse participants described damage in a context of not being able to do what they know is right for the patient, thus they felt that the patient was not getting the care they deserved or would want. SICU nurses described experiences of morally distressing patient care situations that occurred frequently, and influenced their perceptions of the quality of their patient care.

Nurse participants were able to recount multiple experiences in the SICU that were morally distressing because they perceived that patients did not get the care they deserved and the nurse participants were unable to influence the outcome - thus, moral residue developed over time and left the nurse participants with unresolved past experiences. The nurses' experiences of moral distress highlight the significance of this study.

However, several charge nurse participants' described their past experiences of moral distress and harnessed those feelings of negativity and powerlessness to advocate for the patient. For example, charge nurse participants discussed several patient care scenarios where they described interactions where the physicians "rounded" on the patients and determined goals of care and treatment courses. During one of these interactions, the patient did not feel that the attending physician was respecting his treatment decisions. Thus, the nurse participant stood up for the patient in-front of the entire trauma team of physicians. 
Specific Aim 1 was addressed through the identified process of moral distress, moral residue, and moral crescendo. To the best of this researcher's knowledge, this is the first study to capture the process of moral distress compounding into moral residue and subsequently resulting in the crescendo effect among adult SICU nurses in a Level-1 trauma center. Thus, this novel study has been illustrative of the SICU environment's struggle to maintain nurses' presence at the bedside, particularly in such a caustic and stressful environment. In light of the five themes identified in this study, this process illustrates not only how nurses become morally distressed, but also highlights the steps nurses take to mitigate the stress and feelings of inadequacy from these patient care encounters. Thus, this identified process is vitally important in describing the initial morally distressing patient care event. More importantly, it illustrates that moral residue and moral crescendo may be forthcoming; however, there are steps each nurse participant took on their own volition to mitigate the spread of powerlessness. According to Dudinski (2016), the experience of moral distress is marked by concern for the patient's well-being while also an accompanying and burdening sense of powerlessness. Moreover, Hamric et al. (2012) notes that moral distress is a negative experience and manifests as powerlessness, anger, and frustration. Thus, research supports that some of the essential attributes of the moral distress experience are marked by negative feelings, powerlessness, conflicting loyalties, and uncertainty (Dudzinski, 2016; Hamric et al., 2012; McCarthy \& Gastmans, 2015; Russell, 2012; Savel \& Munro, 2015).

To understand the moral distress among nurses in the SICU environment, data must be aggregated across interviews in order to comprehend why SICU nursing may be toxic to nurses' mental well-being over time, unless steps are taken to cope in a healthy 
manner. In the SICU environment, nurse participants noted numerous patient care situations that resulted in damage to their thinking about how patient care "ought to be". Due to these hallmark experiences of moral distress, moral residue, and moral crescendo - this phenomenon represented a situation that SICU nurses had to combat individually, unfortunately without the support of administration or management. Thus, an unexpected finding is the sheer fortitude and empowerment of these SICU nurse participants to "do the right thing" for their patients. This is a basic definition of moral distress that is supported by research from the coinage of the original term over 30 years ago. For example, according to Andrew Jameton (1984), moral distress is when one knows the right thing to do, but institutional constraints make it nearly impossible to pursue the right course of action. Similarly, the tendency to experience moral distress reflects the moral values and personal moral integrity of the nurse, because these characteristics reflect that the nurse knows the right thing to do and has clarity about their own personal values (Dudzinski, 2016).

Even though nurse participants encountered frequent morally distressing patient care experiences, the nurse participants were still able to identify why the patient care situation was morally or ethically wrong. Importantly, this highlights the interesting characteristic about moral distress research and what sets it apart from other phenomenon such as compassion fatigue, burnout, and PTSD, which are not the result of a moral component not being fulfilled (Epstein \& Hamric, 2009). Thus, what sets morally distressing experiences apart from other concepts is the presence of a moral wrong in the context of patient care that prevents the nurse from doing the right thing for the patient, even though they know what should be done. Thus, the corrective action is known, but 
unable to be delivered by the nurse due to circumstances beyond their control, such as the physician's exercising of a power differential over the nurse, or a family member that wants to keep treating their loved one - even though the prognosis is poor. Thus, according to Epstein and Hamric (2009), the hallmark of moral distress is that the nurse recognizes and is unable to effectuate change around the patient's care - unable to do the right thing, because they are prevented from doing representing a violation of their core values and duties.

Interestingly, the above description seems dismal and bleak for SICU nurses. However, in contrast, the SICU nurse participants in this study were positive, caring, and the interviews highlighted numerous instances of patient advocacy because that is how these strong-willed SICU nurse participants were able to come back to work every day: through survival, coping, and self-preservation. Subsequently, the five identified themes throughout the data illustrate the broader process of moral distress, moral residue, and moral crescendo. Once this hidden process was uncovered, it was clear that the nurse participants were not at fault - they were not to blame. Instead, the SICU nurses were operating in an organization that did not support their perceptions and experiences of moral distress at the organizational, unit, or individual level. Similarly, Hamric (2014) conducted a case study of moral distress regarding a neonate and the family that was not able to visit frequently, or even be partners in their child's care, due to the prolonged hospitalization. They exhibited numerous instances of moral distress, feelings of powerlessness to stop futile and aggressive care of the neonate among the NICU staff. Interestingly, from this case study, Hamric (2014) noted that a combined approach to 
addressing moral distress and moral residue is warranted, one that combats moral distress at the individual/nurse, team/unit, and organization/system-levels.

Based on these findings, a hospital organizational-wide moral distress consultation service (MDCS) was implemented to acknowledge and address moral distress (Hamric \& Epstein, 2017). Hamric and Epstein (2017) found that the need for institutional efforts to address moral distress was a result of high levels of moral distress reported among two nursing units. According to Hamric and Epstein (2017), their moral distress consultation service initially focused on education and creating a safe space for ethical conversations, but noted that attendance at monthly sessions was labile and there was minimal evidence of institutional impact. Thus, Hamric and Epstein (2017) confirmed the need to acknowledge and address moral distress similar to this study. However, it is unclear from an organizational perspective what would be the best way to address and acknowledge moral distress and moral residue in the practice setting. The process of moral distress development calls into question the moral integrity of nursing practice because the nurse is not able to do the right thing for the patient either through action or inaction, which is not a fault of the nurse. Thus, this study confirmed the significance of moral distress research. The SICU nurses are operating in a system that treats them as expendable care providers.

Theme 1 - Concentrated Caring in High Stress Environment_- "Doing Your Best". Concentrated caring was identified as the first theme, because nurses evaluated the quality of their patient care as "doing their best" for the patient. Thus, the primary subtheme subsumed within theme one is "doing your best" for the patient. Furthermore, in regards to the sub-theme in Theme 1, it was noted from numerous nurse participants that communication with families at the end-of-life was a problem for physicians. From the 
interviews with nurse participants, it was discovered that physicians often obfuscated the true prognosis of the patient in an effort to get the family to agree to the treatment plan that they developed — absent the nurse's input. Hence nurses noted manipulation using multiple teams to communicate with family that was confusing and did not allow them to make an informed decision because specialty physician teams only take responsibility for their specialty and do not readily have general goals of care conversations with the patient and/or families.

The SICU nurse participants in this study felt that in doing their best for the patient, they were able to care for them and communicate with the patients' families when they were near the end-of-life. With respect to communication with families of patients near the end-of-life, nurse participants in this study also felt that physicians "pushed an agenda". Morally distressing experiences were evident in numerous nurse participants, and even occupied a sub-theme in Theme 1: communicating with families of patients near the end-of-life. Pushing an agenda by physicians in the form of communication in order to achieve what the healthcare team wanted for the patient, made the nurse participants feel ignored and disregarded what the families wanted for their loved one. Similarly, according to Woods et al. (2015), the situations that most frequently caused moral distress were (a) providing less than optimal care due to management decisions; (b) witnessing patient care quality suffer due to lack of provider continuity; and (c) working with other healthcare workers who are less competent. Furthermore, the most morally distressing experiences were a result of: (a) working with others who are unsafe or incompetent; (b) witnessing poor quality of care due to poor communication; and (c) seeing patients suffer to due to lack of provider continuity. 
Similarly, Choe et al. (2015) also noted that nurse participants expressed ambivalence towards unnecessary medical treatment, because nurse participants felt that it would be better for older patients with a poor prognosis to spend their final moments at home rather than in the critical care unit further prolonging their life unnecessarily. Likewise, Choe et al. (2015) noted that morally distressing patient care situations primarily occurred in end-of-life situations. In contrast, the suffering of the patient was highlighted more by the nurse participants in this study, particularly as they struggled with the inner turmoil from their experience of moral distress as they realized the patient was not getting what they needed and they were powerless to effectuate change.

In this study, nurse participants with less SICU experience felt guilty about interactions with family members. Similarly, an error of judgment or some personal failing reflects the internal constraints of moral distress, such as lack of courage or experience, which leads to feelings of guilt (Dudzinski, 2016). For example, the greatest sense of moral distress for some SICU nurses were interactions with family members when they were fairly new and did not have enough experience to inform the family appropriately. Thus, this lack of ability to assuage family's fears and facilitate their decision-making was morally distressing for nurse participants with less SICU experience.

Similarly, Theme 3: dilemmas resulting from nurses' limited autonomy in treatments was identified by Choe et al. (2015) because nurse participants had limited autonomy in decision-making when they disagreed either with physicians and/or family regarding treatment decisions. Furthermore, Choe et al. (2015) revealed that physicians provide patients and families with incomplete and inaccurate information and nurses felt 
helpless as a result. Thus, findings from this study supports Choe's et al. (2015) results in that physicians do not always provide the best form of communication to patients and their families, thus impacting quality patient care. Similarly, according to Epstein et al. (2016), by acknowledging morally distressing experiences, relief has been the most common response and is not a sign of innate weakness, or cowardice. Instead, according to Epstein et al. (2016) acknowledging moral distress on the part of the provider is a sign of caring, competence, and commitment to patient advocacy. The complexity of SICU patients and the stress involved in their care, created a high stress environment for the nurses.

Nurse participants in this study demonstrated ambivalence toward prioritizing tasks over human dignity similar to nurses as described by Choe et al. (2015), when nurse participants described feeling uncomfortable when work-related processes prevented them from advocating for patients' autonomy or respecting their human dignity. The authors cited processing of a do-not-resuscitate order and preemptively preparing death paperwork, before the patient died, merely to expedite the process. Interestingly, nurses took issue with this because they felt like they were not respecting human dignity.

Additionally, according to Johnson-Coyle et al. (2016), the ICU is a complex work environment where multiple healthcare professionals collaborate, communicate, and educate interprofessionally and also among chronically and acutely ill patients. However, the nurse participants still did their best to provide care for patients, and communicate with families of patients near the end-of-life, too. Importantly, nurses did identify morally distressing clinical situations, and the subsequent suffering the patients endured, and the inner turmoil the nurse felt as a result. Similarly, one of the themes 
identified by Choe et al. (2015) in their phenomenological study of moral distress among ICU nurses found that a main theme was suffering resulting from a lack of ethical sensitivity. The first theme identified in this study highlighted the first part of the process of moral distress, which is the complexity and severity of SICU patients and the stress involved. The patient was the focus of nurse participants despite the maelstrom of stressful events in the SICU environment. Similarly, prior research confirms that moral distress is a phenomenon impacting SICU nurses. For example, Whitehead et al., (2015) determined that ICU nurses, compared to non-ICU nurses, had higher levels of moral distress than physicians and other indirect patient care providers.

Theme 2 - Advocating for Patients \& "Doing the Right Thing". Patient advocacy highlighted the nurse participants' moral distress stemming from issues concerning patient care, especially when they believed that they could not do what was right for the patient. Moreover, the physician-nurse power differential was identified as a source of moral distress for nurses when physicians manipulated families in their decision-making through communication and decision-making. Similarly, Epstein and Hamric (2009) noted that healthcare systems harbor hierarchies, such as a physician giving orders to a nurse to continue care in which they perceive is torturous. Thus, this exploitation of the nurse-physician relationship is a power differential that contributes to moral distress and moral residue and subsequently damages the nurse personally and professionally (Epstein \& Hamric, 2009).

In this study, Theme 2 revealed a sub-theme of physician-nurse power differentials. For example, numerous nurse participants noted the power that physicians hold within healthcare and explained that particular physicians used this power to coerce 
patients, families, and nurses in conversations about goals and plans of care. Similarly, Choe et al. (2015) revealed conflicts with physicians and critical care nurses felt that they worked with physicians who were indifferent to professional ethics and often were not truthful to patients. Similarly, in this study nurse participants were distressed when they witnessed dishonesty and a lack of responsibility in the physicians. For example, SICU nurse participants in Theme 2 - advocating for patients and "doing the right thing" noted the physician-nurse power differential sub-theme. This sub-theme was characterized by physicians who use their status and position in the healthcare context as leverage to intimidate nurses and coerce families into decisions that they may not readily make on their own in the absence of the physician's perspective. Thus, it was morally distressing for nurse participants in this study to encounter physicians who would selfishly promote an agenda with goals of care and not allow the patients and/or families to make a decision that was personal and thought-out.

According to Manara et al. (2014), the best solution for moral distress is one that considers the decision-making power of all those involved in the clinical situation (i.e., patient, family, nurse, and other healthcare professionals), while also being aware of the values and duties involved, because they are often varied and diverse. The major subtheme encapsulated within Theme 2 was positive nursing - "doing the right thing". It was surprising to find so many nurses that were morally distressed, but also maintained a positive outlook and were able to pinpoint the violations to their moral values accurately in each interview. The positive nursing aspect represented a dual meaning with nurse participants maintaining a hopeful, positive outlook in spite of their moral distress. Similarly, the CERN project espoused to maintain open communication with staff about 
ethical issues and they noted a positive impact in improving the quality of patient's care and alleviation of the moral distress experienced by the nurses (Robinson et al., 2014). Moreover, positive nursing was about doing the right thing for the patient, even if it was not a popular idea among other healthcare professionals.

Themes 1 and 2 of this study identified the core values and duties of a nurse that allow them the ability to recognize moral distress. For example, Themes 1 and 2 focused on doing one's best and also doing the right thing for patients in order to be an appropriate advocate, which is a part of a nurses' core values and duties. Similarly, the central theme of morally distressing experiences is that they violate one's core values and duties as a nurse caring for sick and vulnerable patients (Epstein \& Hamric, 2009). However, nurse participants maintained positivity and were able to name clinical situations as violations to moral values due to their empowerment as survivors of the trauma. In contrast, Choe et al.'s (2015) identified themes do not focus on these positive aspects, but instead highlight the negative aspects of moral distress for the nurses.

Theme 3 - Surviving Moral Distress in the SICU Environment-Magnifying Stress \& Self-Preservation represents the magnification of stress for the nurse in the SICU environment over time, thus the nurses have to use self-preservation to survive the numerous sources of moral distress: nursing management problems, short-staffing, lack of resources, incompetent RNs lacking SICU experience, and the demands of additional charting and other responsibilities that nurse participants felt should fall under the responsibility of management — not bedside nurses who were responsible for patient care. Interestingly, previous research confirms this negativity. According to Choe et al. (2015) their first theme was ambivalence towards treatment and care. This closely resembles 
moral residue identified in this study, because unresolved feelings that occurred repeatedly (i.e., moral residue) caused nurse participants to sacrifice their quality of care. For example, nurse participants in this study reported that they did not work as hard as an SICU nurse compared to when they first started. Interestingly, Choe's et al.'s (2015) Theme 1 was composed of the following sub-themes: ambivalence towards prioritizing tasks over human dignity, ambivalence towards unnecessary medical treatments, and ambivalence towards the compulsory application of restraints.

Nurse participants in this study perceived that administration and management did not care or support them with resources as bedside level nurses. For example, nurse participants cited short staffing as a major concern to illustrate this lack of advocacy for staff on the part of management and administration. Repeated examples were recounted of nightshift nurses working with only four nurses instead of the standard of five nurses when 10 critically ill patients needed care. Similarly, Choe et al. (2015) noted another major morally distressing situation among critical care nurses was the inability to provide comprehensive care due to short staffing and this made the nurse participants feel powerlessness and resentful towards the hospital.

Nurse participants discussed situations where SICU nurses had to take three patients, particularly when they just started in SICU and were new nurses, or were pulled from SICU to staff other ICUs that were short-staffed, or were assigned as relief charge nurse positions for which they were not ready or prepared. Thus, short staffing was blamed on management and administration for not advocating for staff through provision of resources. This caused severe conflict and insurmountable moral distress among the nurse participants because not only did they feel like they could not provide adequate 
care to their patients, but they felt powerless to stop the vicious cycle of unethical behavior on the part of management and administration. Importantly, Epstein and Hamric (2009) clarified that morally distressing experiences occur because poor unit/team performance prevents interdisciplinary communication and collaboration, and healthcare organizations lack policies and leadership that acknowledge and validate moral distress and moral residue among nurses.

Moral distress, with respect to the SICU environment, magnifies the nurse participants' stress and suffering, requiring the nurse participants to have to resort to selfpreservation in order to survive. Providing end-of-life care to patients may be the most morally distressing event for nurses, because of the potential for disagreements (TaylorFord, 2013). Highest levels of moral distress were reported in situations related to aggressive, non-beneficial, futile treatment at the end-of-life (Wilson et al., 2013). According to St Ledger et al. (2013), the main goal of ICU treatment "is to help patients survive acute threats to their lives” (p. 1870). As this SICU study demonstrated, nurse participants feel differently about this prior statement, for example, nurse participants in this study cited aggressive treatment at the end-of-life as a major contributor to their moral distress and violations to their core values and duties. Similarly, difficult decisions about de-escalating care or moving to more palliative measures must be had (St Ledger et al., 2013). Particularly, the SICU may perpetuate a moral gray zone as a result of advanced healthcare technologies and treatments that postpone life without accounting for any quality of life as this study demonstrated.

For example, this SICU study noted problems with nursing management not being supportive of bedside SICU nurses, staffing was always short, and resources were 
lacking. Subsequently, a major sub-theme within Theme 3 was nursing management problems, short-staffing, and lack of resources, because these attributes named by the nurse participants were more likely to contribute to moral distress and subsequent moral residue if left unaddressed. It was also noted that staff worked with nurses considered incompetent because they lacked SICU experience, and it took time for them to learn their role. Interestingly, the most common root cause or source of moral distress and moral residue were clinical situations related to end-of-life care. For example, nurse participants cited instances ranging from too aggressive or too minimal care.

Comparably, Epstein and Hurst (2017) noted that nurses may experience moral distress related to following the family's wishes to continue treatment not in the patient's best interests, poor communication, lack of continuity of care, short-staffing, working with incompetent and/or unsafe providers, and providing sub-optimal care because of pressure to reduce costs by management. Similarly, Epstein's (2008) dissertation research noted that all instances of moral distress in her study were related to clinical situations of prolonged, aggressive treatment at the end-of-life that was perceived to be futile.

Interestingly, Theme 3 in this study encompassed concerns about management. In Theme 3 of this study, nursing management problems were highlighted along with shortstaffing, and lack of resources. Interestingly, it was found that nursing management and administration did not advocate for the nurse participants in this study. For example, nurse participants felt that administration, management, and stakeholders away from the bedside were making decisions about how care should be provided at the beside.

Similarly, Choe et al. (2015) identified in their study, that misguided, administrated policy morally distressed the nurse participants. For example, nurses were 
morally distressed when the financial concerns of the hospital trumped the respect for patient care, human life, and human rights (Choe et al., 2015). Similarly, the future goal is for organizations to support the foundation for the development of research interventions to create safe and reflective moral spaces that concentrate on the ethical dimensions of practice (Hamric \& Wocial, 2016). In particular, charge nurse participants noted numerous clinical situations that were the results of administration and management not staffing the SICU units adequately and efforts to save money and put more responsibility on nurses at the bedside - rather than administration. In this study, nurse participants were frustrated and morally distressed by the fact that they did not feel supported by management, administration, the nursing director, or the SICU nursing manager.

In addition, Robinson and Stinson (2016) used a phenomenological qualitative design to explore eight nurses' experiences with moral distress. A major theme identified by the researchers was asking God for forgiveness, because nurses expressed regret for care they felt "forced" to provide, thus these nurses wanted to be forgiven. Thus, Theme 3 in this study highlighted the major morally distressing issues nurse participants experienced and not surprisingly guilt was prevalent. Similarly, previous research demonstrated that moral distress is characterized by frustration, guilt, physical symptoms (e.g., fatigue, lack of sleep, lack of appetite, diarrhea, vomiting), and anxiety related to repressed or violated moral values and beliefs (Austin et al., 2005; Gutierrez, 2005). Central to the experience of moral distress is the violation to one's core values and duties. Thus, it was particularly important that previous research noted that nurses felt "forced" to provide treatment. Similarly, this study's nurse participants demonstrated a 
corroboration of the power differential that leads to action or inaction on the part of the nurse as they are witnessing ethical violations unfold at the bedside.

Theme 4 - The Stain of Moral Residue-Surviving \& Coping with the Impact on Integrity \& Values revealed that nurse participants who experience numerous morally distressing clinical situations eventually develop moral residue. Theme 4 - the stain of moral residue - surviving and coping with the impact on integrity and values sub-theme: nurses "just punching clock" supports Theme 2 from Choe et al. (2015). The second theme identified by Choe et al. (2015) was suffering resulting from a lack of ethical sensitivity, which applied to nurses who did not follow the proper nursing standards of care, and moral distress occurred when they witnessed fellow nurses not working to the best of their ability by being ethically insensitive, lacking a caring attitude, and trying to cover-up unethical behavior.

For example, in this study SICU nurse participants bemoaned other nurses who did not "care as much as they did" and this made them wonder why they tried so hard to care for patients when peers were not providing their best patient care. This led to moral distress in SICU nurse participants because they felt that they were alone in trying to provide the best possible care to the patient, questioning why they should try as hard when those around them did not appear to care at all. Furthermore, this study found that nurse participants tried to help fellow nurses in certain patient care situations that were morally distressing, but since they were only observing the situation unfold, and not actually taking care of the patient, they were unable to effectuate any change - even when they made suggestions to the nurse based on their observations. 
Moral residue develops as a result of unresolved feelings and emotions associated with numerous past morally distressing experiences. Interestingly, this theme was negative, because many nurse participants were frustrated and felt guilty about their morally distressing experiences. According to Choe et al. (2015), nurses noted ambivalence towards the compulsory application of restraints to patients. This is similar to the identification of moral residue in this study, because it is characterized by unresolved feelings that could be considered as ambivalence. In contrast, this study did not find any moral distress among the 21 nurse participants regarding the application of restraints on SICU patients. However, Choe et al. (2015) noted that critical care nurses felt guilt about applying restraints to patients all the time because it robbed them of human dignity, although they felt that during necessary procedures such as intubation or bronchoscopy, it is necessary to have restraints on the patient to prevent interference with lines or procedures. Thus, even though nurse participants in this study did not identify restraints as an issue, they still identified moral residue and the ambivalence they felt about patient care, providing evidence for the damaging impact of moral distress and moral residue.

The experience of moral distress leaves its mark on the victim - in this case, SICU nurses. This theme represented the development of moral residue. The major subtheme here is surviving and coping with the impact on personal moral integrity and values. The nurse participants were frustrated as they experienced moral distress with multiple patients and this developed into moral residue due to the unresolved feelings regarding the clinical situations that were morally distressing. In contrast, Henrich et al. (2017) noted that some healthcare professionals were able to distance themselves from 
morally distressing experiences at work by becoming less invested. This is the opposite of quality patient care. Moreover, Henrich et al. (2017) noted that the ICU culture prohibited the sharing and discussing of emotions, thus staff repressed and hid their morally distressing experiences in order to prevent appearing weak.

Similarly, according to Hamric and Blackhall (2007), nurses' perceptions of moral distressing situations were influenced primarily by the frequency of those events. Moreover, nurses, unlike physicians, cannot leave stressful situations but are instead obligated to stay on the unit with their patients, thus this increased contact to the clinical situation may contribute to perceptions of the frequency of morally distressing situations (Hamric \& Blackhall, 2007). Many nurse participants did not compromise their patient care, but identified particular instances where care was compromised which led to moral distress. Therefore, these SICU nurses were surviving and coping with moral distress, as they perceived threats to their personal moral integrity and values. Moreover, many nurses used the method of compartmentalization to function effectively. Finally, other nurses, and healthcare professionals, were described as "just punching the clock" and not focusing on how they are able to do their best for the patient.

Theme 5 - Moral Crescendo's Coup De Grâce - Suffering \& Wounded - “Cannot Take It Anymore" contained a major sub-theme, which was post-traumatic stress disorder creating an environment where the nurse participant was ready to leave their job. The complexity of the SICU environment and the nurse participants' inability to establish an outlet for their stress over time may lead to a moral crescendo when moral residue has built-up to the point of being ignored and the nurse "cannot take it anymore". According to Epstein and Hamric (2009), hypothesized negative consequences of morally 
distressing experiences include burnout, withdrawal and detachment from the ethical dimensions of practice, conscientious objection, quitting a job, or quitting nursing. Similarly, according to Hamric and Blackhall (2007), 45\% of ICU nurses ( $n=90)$ reported that they had left or had considered leaving a nursing position due to moral distress. Thus, moral crescendo's deathblow to the mentally and psychologically suffering and wounded nurse is manifested as being "burned out" and/or developing PTSD.

The crescendo effect presupposes that morally distressing events occur repeatedly. Epstein and Hamric (2009) revealed several reasons for developing nursing policy at the administrative-level that would work to acknowledge and address moral distress. First, nurses' moral distress is indicative of larger healthcare practice problems, such as poor team communication, non-existent team and patient-family collaboration, and perceived powerlessness from extant power differentials (Epstein \& Hamric, 2009). Second, ethics consultation services must pay particular attention to the crescendo effect so nurses experiencing emotional reactions are not blamed (Epstein \& Hamric, 2009). However, it was important not to blame nurse participants for this experience, but rather support them. The SICU nurses' morally distressing experiences indicated that moral issues underpin their perceptions that something is wrong. Third, moral distress affects direct and indirect healthcare professionals, which is further evidence this phenomenon extends to the unit/team, and organization/system levels, hence comprehensive multidisciplinary interventions are required (Epstein \& Hamric, 2009).

According to Robinson et al. (2014), the Clinical Ethics Residency for Nurses (CERN) project demonstrated qualitative evidence for acknowledging and addressing 
nurses' moral distress, because they revealed a positive effect on patient care as evidence by the shared narratives provided by study participants. Similarly, Choe et al. (2015) used Giorgi's phenomenological approach to explore perceptions of morally distressed ICU nurses $(N=14)$ caring for adult patients identifying five themes: (a) ambivalence towards treatment and care, (b) suffering resulting from a lack of ethical sensitivity, (c) dilemmas resulting from nurses' limited autonomy in treatments, (d) conflicts with physicians, and (e) conflicts with institutional policy.

The future goal is to provide the foundation for the development of research interventions to create safe and reflective moral spaces that concentrate on the ethical dimensions of practice (Hamric \& Wocial, 2016). This study supports the idea from Hamric and Epstein (2017) that unit- and institutional/organizational-buy-in is necessary in order to support systemic level change in addressing moral distress. Hamric and Epstein (2017) developed a moral distress consultation service aimed at addressing moral distress through an institutional mechanism. Moreover, they discovered the following five themes from 23 interview consultations with healthcare professionals aimed at developing and evaluating a consultation service as a viable option in addressing moral distress: acknowledgement of staff concerns; staff empowerment; staff engagement; improved team collaboration; and unit- or organizational-level change. For example, Hamric and Epstein (2017) created a hospital-wide Moral Distress Consult Service that acknowledged and validated healthcare professionals' moral distress. Recommendations for addressing moral distress were discussed and a meeting summary was provided to the manager. If warranted, the case was sent to the ethics committee. Policy should focus on 
services available to nurses and healthcare professionals that recognize, validate, and mitigate their moral distress.

Hamric and Epstein's (2017) goal with the moral distress consultation service was not to completely remove moral distress in a particular clinical situation. The service was aimed at empowering healthcare professionals to use the resources available to them in order to reach an appropriate resolution. The findings of this study support the idea of Hamric and Epstein (2017) that moral distress will not be completely eradicated by future research.

Creating substantive change in practice requires acknowledging and addressing root causes of moral distress (Thomas \& McCullough, 2017). According to Sabin (2017), moral distress ought to be used for improving organizations. The AACN published a policy statement online that called for nursing programs to address and alleviate negative consequences of moral distress while also creating a healthy work environment (AACN, 2008). Creation of a healthy work environment is synonymous with an ethical workplace where nurses feel comfortable identifying and discussing ethical issues. Fostering an ethical climate/healthy workplace environment is consistent with the social determinants of health (Mason et al., 2016), the Triple Aim of a value-based healthcare system, the Affordable Care Act (ACA), the Institute of Medicine's (IOM) (2011) future of nursing goals, the ANA Code of Ethics for Nurses (2015), and the International Council of Nurses (ICN) (2011) Code of Ethics for Nurses. A revised combined policy statement of moral distress from professional organizations, such as the ANA and American Association of Critical Care Nurses (AACN), is necessary because influential bodies and professional organizations may influence administrative and legislative stakeholders to 
promote and implement comprehensive moral distress policy that impacts the organization's ethical work environments. Policy with influential support from credible organizations empower hospitals to adopt change aimed at the individual/patient, unit/team, and institutional cultures.

\section{Clinical Situations}

Numerous clinical situations created moral distress and moral residue among SICU nurses in this study. This extends previous research by Hamric et al. (2012) in their development and validation of the Moral Distress Scale-Revised (MDS-R) instrument found that the major root causes of moral distress are the result of clinical situations, internal constraints, and external constraints. For example, as described by Hamric et al. (2012) and corroborated by this study, some clinical situations that caused moral distress in the nurse participants included: providing unnecessary / futile treatment, prolonging the dying process through aggressive treatment, inadequate informed consent, working with caregivers who are not as competent as care requires, lack of consensus on a potential treatment plan, lack of continuity of care, conflicting duties, inappropriate use of resources, providing care that is not in the best interests of the patient, inadequate pain relief, providing false hope to patients and families, hastening the dying process, lack of truth-telling, and disregard for patient wishes. All of these clinical situations that were identified as root causes of moral distress used in the conceptual and operational development of the MDS-R (Hamric et al., 2012) and were corroborated by this study.

\section{External Constraints}


Specific external constraints were identified by Hamric et al. (2012) and supported by this study, including: inadequate communication among team members, differing perspectives among healthcare team members, inadequate staffing and turnover, lack of administrative support, policies and priorities that conflict with patient care, following family wishes of patient care for fear of litigation, tolerance of disruptive and abusive behavior, compromising care due to pressures to reduce costs, healthcare hierarchies, lack of collegial relationships, nurses lack of involvement in decisionmaking, and compromised care due to insurance pressure or fear of litigation. External constraints are important because they typically describe root causes of moral distress that are out of the nurses' control.

\section{Internal Constraints}

Nurse participants were motivated by internal constraints in their experience of moral distress. For example, some nurse participants were not able to effectuate change because they felt like they did not know enough, or have enough SICU experience. Other nurse participants felt powerless and thus were unable to make a difference in the patient's care or outcomes as the morally distressing event was unfolding. Thus, this study corroborates prior research regarding the feelings and emotions the individual experiencing the moral distress encounters personally including: perceived powerlessness, inability to identify ethical issues, lack of understanding regarding the full situation, self-doubt, lack of knowledge of alternative treatment plans, increased moral sensitivity, lack of assertiveness, and socialization to follow others (Epstein \& Hamric, 2009; Hamric et al., 2012; Hamric, 2014). This study extended the internal constraint concept by pinpointing those who experienced a moral crescendo. 
In this study, moral issues that created moral distress and moral residue, or repeated instances of moral distress that are left unresolved and remain as a stain to one's own personal moral integrity and values, included communication with families of patients near the end-of-life, physician-nurse power differentials that resulted in the physician using power and status to coerce the sequence of events in a clinical encounter to correspond with their agenda. Interestingly, this study corroborates the MDS-R's classification of root causes of moral distress by noting some common sources: providing unnecessary/futile treatment, inappropriate use of resources, inadequate communication among team members, lack of administrative support, and hierarchies within healthcare/differing interprofessional perspectives among nurses and physicians regarding goals and plans of care (Hamric et a., 2012). For example, nurse participants noted physicians who were not completely truthful with families, or were manipulating the circumstances through controlling the flow of information to patients, their families, and even the nurses providing their care. Furthermore, other moral issues that caused moral distress and moral residue in the SICU nurses included nursing management problems, short staffing, lack of resources and supplies needed to provide the best possible care for their patients. Moreover, other moral issues include incompetent nurses lacking in SICU experience, who did not feel confident to provide care for complex patients. Additionally, the demands of additional charting and responsibilities in the SICU cannot be understated, because nurse participants felt that they had tremendous demands of charting. With the additional responsibility of peer chart auditing where nurses had to evaluate two different peers' documentation was morally distressing to the SICU nurse participants because they felt like the time needed to dedicate to these peer 
chart audits was robbing patients of needed care, and they believed this was management's responsibility.

Due to short staffing of other healthcare professionals such as physical therapists and occupational therapists, nurse participants were morally distressed by the additional responsibility of also getting their complex patients up and out of bed with limited staff. Evidence suggests that early mobility of patients in the ICU, particularly among patients on ventilators, results in better functional outcomes upon hospital discharge, a shorter duration of ICU delirium, and more ventilator-free days compared to standard care (Schweickert et al., 2009). Interestingly, nurse participants noted that they were not able to comply with these evidence-based guidelines — such as early mobility — due to lack of staffing, as previous research by Schweickert et al. (2009) has suggested in their randomized controlled trial. Thus, the nurse participants were morally distressed about being unable to provide quality patient care to their patients according to the evidencebased guidelines due to a lack of staffing.

This is a prime example of the importance of bedside SICU nurses' complaints of moral distress, because they indicate broader, systemic problems and insufficiencies in the healthcare organization. For example, Fleck (2009) highlights the stress our current healthcare system is under through limited healthcare resources available to assess and evaluate unlimited healthcare needs.

This study supports the issue identified by Hamric and Epstein (2017) that unitand institutional/organizational-buy-in are necessary in order to support the systemic level change in addressing moral distress with adequate resources. Hamric and Epstein (2017) developed an important resource to combat moral distress, namely, a moral 
distress consultation service aimed at addressing moral distress through an institutional mechanism. Moreover, they discovered the following five themes from 23 interview consultations with healthcare professionals aimed at developing and evaluating a consultation service as a viable option in addressing moral distress: acknowledgement of staff concerns; staff empowerment; staff engagement; improved team collaboration; and unit- or organizational-level change. For example, Hamric and Epstein (2017) created a hospital-wide Moral Distress Consult Service that acknowledged and validated healthcare professionals' moral distress. Recommendations for addressing moral distress were discussed and a meeting summary was provided to the manager. If warranted, the case was sent to the ethics committee. Policy should focus on services available to nurses and healthcare professionals that recognize, validate, and mitigate their moral distress. Furthermore, Hamric and Epstein's (2017) goal with the moral distress consultation service was not to completely remove moral distress in a particular clinical situation. The service was aimed at empowering healthcare professionals to use the resources available to them in order to reach an appropriate resolution. This study supports the idea of Hamric and Epstein (2017) that moral distress will not be completely eradicated by future research.

Nursing participants specifically identified the moral issue - inability to provide quality nursing care to their patients due to lack of staffing. Furthermore, the nurse participants identified why the lack of staffing was a problem: management and administration. In addition, nurse participants noted that even when they tried to address their moral distress by going to upper management, these issues were only resolved temporarily. Instead, they felt that they did not have the support of nursing management 
and administration, which caused the majority of nurse participants to feel powerless and inadequate to effectuate any meaningful change around these moral issues. These findings in this study are similar to those of Epstein and Hamric (2009) who found that the lack of support of nursing management and administration was morally distressing, particularly the lack of an organizational response to even acknowledge the existence of moral distress among staff (Hamric, 2014).

\section{Moral Residue}

Moral residue was identified as an important problem by Epstein and Hamric (2009); however, a there is a dearth of scientific research on moral residue. Moral distress leads to compromised personal moral integrity and moral residue. Moral residue occurs when a nurse's moral distress is unaddressed and the moral and emotional baggage remains (Epstein \& Hamric, 2009). According to Webster and Baylis (2000), moral residue is defined as, "that which each of us carries with us from those times in our lives when in the face of moral distress, we have seriously compromised ourselves or allowed ourselves to be compromised" (p. 218). Moral residue is the lingering experience created from numerous unresolved morally distressing events, which accumulate, pile up, and lead to further distress (Epstein \& Hamric, 2009). This experience is painful because it threatens and betrays deeply held and cherished beliefs and values, thus the experience of moral residue is lasting and powerfully concentrated in one's thoughts (Webster \& Baylis, 2000).

Evidence of moral residue was demonstrated by numerous nurse participants in this study. This study corroborated these findings by identifying moral residue when SICU nurse participants were able to recount multiple morally distressing events over 
their nursing career that were never resolved or processed. This was evident in the interview process, because the nurse participants were frank and open about their powerlessness, as if they were resigned to this fate, and were just trying to function and provide the best possible care for their patients in an organizational structure that they are knowingly aware does not support them.

Moral distress and moral residue impacted nurses' perceived quality of care for adult patients in the SICU in several ways. Hamric et al. (2012) identified three categories of root causes of moral distress in the literature, which informed their development of the MDS-R instrument. Similar clinical situations and internal constraints that were identified by Hamric et al. (2012) as root causes of moral distress also contributed to the moral issues identified by participants in this study. Clinical situations included physician-nurse power differentials, communicating with families of patients near the end-of-life, nursing management problems, short-staffing, lack of resources, incompetent nurses lacking in SICU experience, and the demands of additional charting and responsibilities. This study identified a positive aspect of moral distress and moral residue — many nurses even though severely morally distressed and suffering from the stain of moral residue still managed to do their best and try to do the right thing everyday. Even the nurse participants who voiced that they do not care as much as when they first started working in SICU, still felt guilty about this progression of their feelings about bedside nursing.

Moral distress is a challenge to core obligations and values (Epstein \& Hamric, 2009), including challenges, threats, and violations to personal and professional moral integrity (Thomas \& McCullough, 2015). The core nature of the nurse's values and 
integrity are called into question as a result of internal constraints (e.g., lack of courage, inexperience) and external constraints (e.g., orders, policies), which are perpetuated by broader healthcare systems at the unit/team and organizational levels (Dudzinski, 2016; Hamric, 2014; Thomas \& McCullough, 2015; Varcoe, Pauly, Webster, \& Storch, 2012). In addition, this study revealed that moral distress is a process where subsequent moral residue develops and will eventually crescendo. This study supported the findings from previous literature that moral distress leads to burnout and withdrawal from the ethical dimensions of nursing practice. In the case of withdrawing from the ethical dimensions of practice, many nurse participants compartmentalized. However, in the context of this study, it is evident that compartmentalization used by SICU nurse participants in this study was a necessary response for surviving and coping with morally distressing events that developed into the ever-lasting stain of moral residue, especially in an environment where nurse participants voiced they are not supported by administration, management, and sometimes other physicians and/or nurses. Although this study focused on individual SICU nurses, qualitative interviews illuminated unit- and system-wide problems in the continuum of care that influenced individual SICU nurses' perceptions and experiences of moral distress and moral residue, particularly as they applied to the frequency and intensity of experiences. For example, SICU nurse participants noted that clinical situations that resulted in a perception and experience of moral distress were often frequent and intense. These moral distress experiences were frequent, because SICU nurse participants readily recalled multiple events in detail, and intense because of the "mark" that moral residue left on the individual noted through the vivid imagery SICU nurses used to describe their experiences. 
Theme 2 - Advocating for patients and "doing the right thing" was identified because although some nurse participants' quality of care was impacted negatively by their experiences of moral distress, the majority of nurse participants in this study were positive about their quality of patient care. This positivity is an unexpected finding not identified by prior research, to the best of this author's knowledge. The first sub-theme described the physician-nurse power differential. This sub-theme was negative, because even experienced, intelligent nurses were more passive in the presence of physicians, and because physicians were viewed as authority figures and thus garnered respect. Another sub-theme was patient advocacy. In contrast, other nurse participants stood up and advocated for patients regardless of whether it was another physician, nurse, or other healthcare professional - these particular nurses would advocate for their patient, in front of anyone. SICU nurses are under a tremendous amount of stress in the SICU environment due to the frequent, multiple occurrences of moral distress. Thus, that some SICU nurses are still able to be patient advocates and focus on positive nursing - "doing the right thing" (final sub-theme), is a testament to the SICU nurses' character, resolve, and commitment to the patient. In contrast, it was unexpected that positive nursing "doing the right thing" would be identified in this study of moral distress because as Epstein et al. (2016) noted: dilution of the concept is noted when other concepts are included. However, the nurse participants in this study conveyed this positive perspective when pressed about their experiences - they remained positive and remained at the bedside in the SICU.

Interestingly, some nurses felt that their experiences in the SICU made them want to seek more knowledge and cover all their bases so that they would not make a mistake. 
Arguably, these nurse participants were empowered and strengthened by their experiences of moral distress because it made them better patient advocates because they were more meticulous and hyper-vigilant for fear of getting in a "comfort zone" and making an error. Other nurses felt that the quality of their care was negatively impacted by their moral distress because they were "burned out" which corroborates prior research. (Epstein \& Hamric, 2009; Hamric, 2014; Hamric et al., 2012). Nurses' voices are important because they spend the most time with patients at the end-of-life than any other healthcare professional, thus if nurses feel negatively about a course of treatment then it must be acknowledged, particularly in the critical care adult environment where the margin for error is small (Whitehead et al., 2015). Furthermore, as part of the moral distress experience, patients' quality of care is a primary concern as documented widely in research (Dudzinski, 2016; Epstein \& Hamric, 2009; Hamric, 2014; Hamric et al., 2012; Webster \& Baylis, 2000; Whitehead et al., 2015).

Interestingly, Whitehead et al. (2015) noted that lack of continuity of care and poor team communication are common sources of moral distress indicating that strategies to improve communication and teamwork are an important focus of intervention research. Additionally, this study found that quality patient care was negatively impacted by experiences of moral distress because "doing the right thing" for the patient could not be achieved from the SICU nurse's perspective. Thus, issues of communication were at the forefront, particularly in the context of end-of-life circumstances in this particular study.

Moral distress will never be completely eradicated (Hamric, 2014) due to technological advancements that prolong life and make quality of care determinations difficult. Rather, it was naturally assumed among the nurse participants that moral 
distress was just a characteristic of the job as nurses in the SICU environment, because of the nature of complex patient care and they way incapacitated patients may be allowed to linger indefinitely because of the patient's poor prognosis. As technology continues to advance, healthcare ethics must evolve and keep pace with the gray areas of healthcare practice through a constant exploration of the morality of individual actions and consequences. Thus, this study provides evidence of SICU nurses suffering in the SICU environment. However, if the environment in which moral distress occurs is changed and structures that perpetuate moral distress are challenged, then it is important to identify the immorality of patient care situations at the center of healthcare delivery when considering the ethical dimensions of practice (Epstein \& Hamric, 2009) associated with providing quality care for ailing, vulnerable patients in the SICU.

\section{Influence on Professional Practice}

From this study, moral distress and moral residue influence professional practice standards, personal moral integrity, and values among SICU nurses by preventing them from "doing the right thing" in a patient care situation. Moral distress is a challenge to core obligations and professional values as Epstein \& Hamric (2009) point out in their theoretical paper on the moral crescendo effect, including challenges, threats, and violations to personal and professional moral integrity (Thomas \& McCullough, 2015). Interestingly, Thomas and McCullough (2015) in their philosophical taxonomy of moral distress identified the challenges, threats, and violations to personal and professional moral integrity. In this study, only the impact on personal moral integrity and values was explored because it was unclear how the experience of moral distress impacts SICU nurses short- and long-term. 
The core nature of the nurse's professional values and integrity are called into question as a result of internal constraints (e.g., lack of courage, inexperience) and external constraints (e.g., physician orders, organizational policies), which are perpetuated by broader healthcare systems at the unit/team and organizational levels (Dudzinski, 2016; Hamric, 2014; Thomas \& McCullough, 2015; Varcoe, Pauly, Webster, $\&$ Storch, 2012). Although this study focused on individual SICU nurses, these qualitative interviews illuminated unit and organizational system-wide problems in the continuum of care that influenced the individual nurses' perceptions and experiences of moral distress and moral residue, particularly as they apply to the frequency and intensity of morally distressing experiences. For example, SICU nurse participants when asked about situations where they were not able to "do the right thing" for the patient responded quickly with multiple, vivid examples of patient care situations, providing evidence for moral residue and the subsequent impact on personal moral integrity and values.

According to Theme 4 - The Stain of Moral Residue-Surviving and Coping with the Impact on Professional Integrity and Values, various impacts were noted on the SICU nurse participants' integrity and values in this study. For example, the first sub-theme was surviving and coping with the impact on personal moral integrity and values. Thus, nurse participants were noted to have a strong sense of self-preservation because they remained working in an SICU environment that was stressful, stress-inducing, and immoral due to situations of moral distress that were allowed to persist and occur unchecked. Numerous nurse participants noted positive and negative coping, but the key issue was the necessity of adaptive coping in order to function in this SICU environment. Interestingly, a common finding in this study is that all SICU nurses seemed to practice 
some form of compartmentalization - meaning, they repressed the truly horrible, unethical events in an effort to keep "doing their best" and providing quality patient care to support positive patient outcomes. Thus, even though all processes were failing around them, SICU nurses still made an effort to be able to go to work and provide patient care and remain positive about nursing practice. 


\section{Conclusions}

Exploration of SICU nurses' perceptions and experiences in this study identified moral issues that are of primary concern to SICU nurses: (a) end-of-life issues that cause inner turmoil within the nurse as a result of the patient's suffering; (b) physician-nurse power differential; (c) nursing management problems, short staffing, and lack of resources; (d) incompetent nurses with lack of SICU experience; and (e) the demands of additional charting and responsibilities. The central problem this study investigated was identification of the root causes of moral distress by exploring nurses' perceptions and experiences of moral distress and moral residue in the adult SICU environment and the linkage between moral distress, moral residue, coping, and perceived quality of patient care.

Moral distress and moral residue negatively impacted nurses, because of the violation of their core values and duties as a nurse. This has implications for quality of patient care, and the main obligation of nurses - serving as patient advocates. Because individual nurses cope differently with stress and exhibit different ways of managing their moral distress and moral residue, this study highlights the urgency of exploring how these diverse manifestations of moral distress influence not only their personal wellbeing, but also their perceived quality of patient care. Interestingly, it was learned from this study that there was a lack of consideration for the bedside SICU nurses' well-being, particularly from a management and administrative perspective. Moreover, nurse 
participants cited multiple examples where patient care was potentially impacted by the nurse participants' experiences of moral distress and subsequent moral residue, thus necessitating the need to further understand and address moral distress and moral residue in the SICU environment before crescendo develops and creates burnout in competent, caring professional nurses at the bedside.

Moreover, five themes of moral distress were identified in the SICU nurse participant interviews of this study: (A) Theme 1 - Concentrated Caring in High Stress Environment—-Doing Your Best" comprised of the following sub-themes: (a) doing your best for the patient; (b) caring; (c) communicating with families of patients near the end-of-life; and (d) suffering and inner turmoil; (B) Theme 2 - Advocating for Patients \& "Doing the Right Thing" comprised of the following sub-themes: (a) physician-nurse power differential; (b) patient advocacy; and (c) positive nursing - "doing the right thing"; (C) Theme 3 - Surviving Moral Distress in the SICU Environment-Magnifying Stress \& Self-Preservation with the following sub-themes: (a) nursing management problems, short staffing, and lack of resources; (b) incompetent RNs - lack of SICU experience; (c) demands of additional charting and responsibilities; (D) Theme 4 - The Stain of Moral Residue - Surviving \& Coping with the Impact on Integrity \& Values, with following sub-themes: (a) surviving and coping with the impact on personal moral integrity and values; (b) compartmentalization; and (c) nurses "just punching clock"; and (E) Theme 5-Moral Crescendo's Coup De Grâce—Suffering and Wounded-“Cannot take it anymore" with two sub-themes: (a) burnout; and (b) cannot take it anymore ready to leave. 
These five themes illustrated the process of moral distress, moral residue, and the crescendo effect among the SICU nurses. This process describes how moral distress develops and then the subsequent stain of moral residue that ensues and eventually leads to the coup de grâce, crescendo. The SICU environment is comprised of complex patients that are stressful to take care of because the margin of error is small. The process of moral distress is comprised of multiple patient care experiences within the SICU environment, which are marked by morally distressing events. If the morally distressing patient care experience is the key and occurs frequently, then the stain of moral residue results, because when these morally distressing events go unaddressed, accumulate over time, and the nurses' feelings that are attached with these events go unresolved, the crescendo effect results.

Caring in a high stress environment meant that nurse participants did their best. Thus, the complexity of the SICU patients along with the concurrent stresses the nurse participants experienced as a result illustrated this Theme 1. Advocating for patients and doing the right thing encapsulated Theme 2 . Nurses recognized the negativity of moral distress through physicians who leveraged their power differential to coerce families, patients, and nurses in goals of care discussions. However, the nurse participants remained patient advocates and did their best to always do the right thing for their patients. Interestingly, these first two themes reflect the positivity that was a surprising discovery in this study, particularly among conversations of moral distress and moral residue. The expectation was that the interviews would be negative and gloomy. Yet, nurse participants showed a great deal of self-preservation and survival. Interestingly, the remaining three themes highlight this specific impact on the individual. 
Theme 3 was about surviving moral distress in the SICU environment, which was magnified by psychological stress and also the needs to self-preserve one's own identity. For example, the SICU environment magnifies stress, thus self-perseveration is a necessary coping skill. Furthermore, the nurse participants highlighted numerous individual-level sources of moral distress: nursing management problems, short staffing, lack of resources, working with incompetent nurses lacking in SICU experience, and the demands of additional charting and responsibilities from management. The nurse participants were impacted negatively by these root causes of moral distress and the overwhelming impact is frustrating and feelings of powerlessness to make change.

Theme 4 illustrated the stain of moral residue for the individual. This was another surprising finding in the study - the fact that so many SICU nurse participants suffered from moral residue, which are essentially unresolved feelings associated with a former morally distressing clinical situation. Theme 4 highlights the survival skills that are required by the SICU nurse to cope with the impact to one's personal moral integrity and values through violations of core values and duties as a nurse. Experienced nurse participants learned to compartmentalize their thoughts and emotions about events they felt frustrated and powerlessness to change. In addition, the nurse participants were still attuned to other nurses who did not care as much and were simply "just punching the clock". Thus, the nurse participants' situational awareness was high. Furthermore, these same nurse participants were able to assess and evaluate other healthcare disciplines within the same organization, for example, stating the shortage of physical therapy and occupational therapy services on the weekend was frustrating for many nurse participants. Finally, Theme 5 represents the crescendo effect. The final theme represents 
the nurse participant who "cannot take it anymore". Thus, the outcome on the individual is one of burnout and being unable to handle the clinical environment.

Addressing moral distress and moral residue has clinical significance, because the negative consequences affect SICU nurses' ability to practice and influence the quality of patient care. The interviews conducted from this study highlighted the experiences of SICU nurses that were morally distressing and influenced the quality of their nursing care and their ability to continue to practice. This research demonstrated that moral distress is a significant problem for nurses and the profession of nursing. In particular, this problem is illustrated via the process of moral distress, moral residue, and moral crescendo among SICU nurses. The results of this study are significant because they demonstrate the pervasive impact of moral distress and moral residue among nurses in the high stress SICU environment. The significance of addressing moral distress is not only to make nursing practice more ethical, leading to improved patient outcomes and quality of care, but also to promote nurse well-being, retention, and better prepare nurses with strategies for coping with moral issues in the SICU environment.

This study is significant because it explored moral distress, moral residue, stress and coping, and perceptions of quality patient care among adult SICU nurses in two SICUs. Moreover, to the best of this author's knowledge, this is the only study to document the process of moral distress, moral residue, and the crescendo effect. For example, moral residue is difficult to categorize and a rarely explicitly studied topic in the research literature. However, this study found that nurse participants suffer greatly from moral residue. Their unresolved feelings and emotions about violations to their core values and duties have in fact become a part of their everyday practice in the SICU 
environment, because morally distressing clinical situations are commonplace in a Level1 trauma, academic facility. A major contribution of this study is the extension of moral distress research by identifying not only the key processes of the development of moral distress, but also of moral residue and the crescendo effect as each of these three concepts apply to nurses in the SICU environment.

This study explored the perceptions and experiences of moral distress and moral residue among individual adult SICU nurses to understand how the phenomenon first manifested and was subsequently managed. This is particularly important because this sample of SICU nurses demonstrated a lack of constructive coping with moral distress. Interestingly, this study focused on how moral distress was addressed professionally and personally. However, the focus was on the nurse participants' responses to morally distressing unethical behavior in the SICU environment, not whether (or not) their coping was maladaptive or negative. As a result of this study and the methods employed, it is clear that nurses' experiences of moral distress are a symptom of moral and ethical violations of core values and duties occurring at the bedside in the SICU environment.

\section{Limitations}

A major limitation of this research is the timing of individual interviews. It was difficult to conduct the interviews to allow adequate time between interviews, because once word spread around the SICUs that interviews were being conducted, the researcher was contacted by other nurses so frequently that it was difficult to manage the volume of responses in light of also trying to manage a journal, transcription of audio recordings, memo of notes, and further details to aid in the research process, such as recognizing themes and drawing connections among the data. However, the researcher transcribed 
each individual interview once the interviewing process was completed. Transcribing the interviews from beginning to end in this manner facilitated data analysis. In retrospect, data collection was facilitated by the eagerness of the nurse participants to discuss their clinical perceptions and experiences. However, it was difficult to adhere to the predetermined plan—such as transcribing each interview immediately afterwards and beginning the memoing process concurrently. This also may be a limitation of the researcher's novice approach to qualitative research and interviewing.

Another limitation was asking a nurse participant how they would respond in a hypothetical circumstance and not receiving a truly genuine answer, because the context and circumstances could be different. Thus, responses to hypothetical questions ought to be viewed with some healthy skepticism because some questions asked may have represented scenarios the nurse participant may have not actually encountered. Thus, it would be more difficult for a relatively new SICU nurse to respond to a hypothetical clinical situation they had never encountered and asked what would it take for them to leave an organization over a threat to their personal and professional integrity and values. A final limitation of this qualitative study is that the results are specific to one academic health center with Level-1 trauma SICUs. The results are not transferable to other healthcare organizations either inside or outside the United States.

\section{Implications}

A potential moral critique of healthcare systems and hospital organizations is that the burnout and turnover of nurses indirectly transfers into poor quality of care for patients. The findings of this study have important implications for nursing practice, education, research, and policy. From this study, it is evident that moral distress 
transcends the nurse's bedside experience in the SICU environment. Researchers, educators, administrators, and clinicians will be able to identify, recognize, and develop interventions to alleviate the negative impact of moral distress and moral residue on nurses. By acknowledging and addressing moral distress and moral residue, a crescendo effect may be prevented. This acknowledgement will perhaps reconcile repeated exposures to unresolved violations to core values and professional duties. Thereby, and

more importantly, this reconciliation has the potential to improve the well-being of nurses in the process and also promote quality of care for the patient in the SICU. Nurse's wellbeing is improved, due to the acknowledgement of violations to core values and professional duties in patient care. Better understanding of how the concept of moral distress and moral residue manifests in nurses in the SICU environment is essential for promoting successful coping and management.

The process of moral distress, moral residue, and the crescendo effect uncovered from this study necessitates further theoretical development, because coping with the stress from repeated violations to one's core values and professional duties is only able to keep nurses at the bedside for a finite period of time. Timing is an aspect of moral distress that is perhaps minimally investigated because seminal challenging clinical situations may impact nurse participants differently and require different coping mechanisms or ways of acknowledging and addressing the experience for the individual as this study demonstrated.

\section{Implications for Nursing Practice}

Moral distress is a negative phenomenon and understanding root causes is important because it is the moral issues that underpin why the nurse experiences distress 
in the SICU environment. For example, one nurse participant in this study said she was burned out and wondered why she should try so hard to provide excellent quality care when those around her did not. These manifestations may provide evidence for the crescendo effect. Moral distress has implications for nursing practice, because this is not just an individual/patient problem. Nurses and other healthcare professionals within units/teams and organizations/systems are accountable to each other and must acknowledge moral distress to prevent the damaging development of consequences from a sustained crescendo effect. In this study, a crescendo effect was evident in two nurse participants hospitalized for mental health treatment due to stress in the SICU environment. In particular, poor quality of patient care, resulting from repeated morally distressing experiences that occur in practice, negatively impact nurses and other healthcare professionals as they cope with moral distress and manage their perceptions and experiences.

\section{Implications for Nursing Policy}

This study's findings will be used to develop unit policy in the SICU addressing and mitigating occurrences of moral distress and moral residue because a common goal is to promote nurses' well-being and to advocate for quality patient care. For example, this study demonstrated that nurses are somewhat withdrawn from the ethical dimensions of their practice through compartmentalization. However, this is a survival mechanism and self-preservation technique in light of the experiences of nurses in the Level-1 trauma care SICU environment. This study revealed evidence for the mandatory participation of hospital organizational leadership, administrators, unit managers, and charge nurses in providing SICU nurses and other direct and indirect healthcare professionals within an 
organization and team/unit with the knowledge, tools, and resources to promote interdisciplinary education, communication, and collaboration to acknowledge and address moral distress, moral residue, and the consequences of participating in moral issues at the bedside. Thus, in order to account for the wellness of bedside nurses working in the SICU, it is necessary for all levels of leadership and administration within the healthcare organization to work together to communicate how to provide better ethical quality patient care.

This study examined nurses' coping abilities and their perceptions and experiences of moral distress, moral residue, and the impact on quality patient care in the SICU environment. The goal was to delineate causes of moral distress and their origins to prevent blaming nurses for their moral distress and to use their distress constructively to acknowledge and address moral issues at the patient's bedside. It is important for SICU patient care units to encourage open and deliberate de-briefing sessions from particularly stressful morally distressing cases. Ways of addressing the negative experience of moral distress, such as through "safe spaces" and de-briefings, may enhance the nurses' wellbeing and the patient's quality of care, but also guides conversations persistently towards reconciling the ethical dimensions of practice with clinical practice in the SICU environment on an ongoing basis.

\section{Implications for Nursing Education}

The implications for nursing education from this study recommend a discussion of ethical issues embedded in morally distressing root causes in nursing undergraduate curriculum. By discussing nursing ethics case studies that incorporate morally distressing scenarios into simulation, nursing educators may begin a conversation with 
undergraduate nursing students about how to communicate with patients, families, and other healthcare professionals regarding the intricacies of complex healthcare and ethical issues, as well as the importance of patient advocacy.

\section{Implications for Nursing Research}

Currently, there are no instruments designed to measure moral residue, and the development of an instrument will be of critical importance in the future, particularly provided the current public health coronavirus (COVID-19) pandemic. The ability to expeditiously and objectively pinpoint an empirical cutpoint number may be helpful in identifying moral distress and moral residue more readily. Ethical concerns in clinical situations, particularly in the SICU environment, are only going to increase in the future. Moral distress is inherent to healthcare as this study demonstrated. The goal is not to focus on eradicating moral distress. Based on the results of this study, it is evident that moral distress and moral residue occur in the SICU environment and the phenomenon needs to be acknowledged and addressed.

Future research is needed to better understand the phenomenon identified in this study - the process of moral distress, moral residue, and the crescendo effect. Thus, the goal of future research will not be necessarily to document the existence of moral distress, because there is overwhelming evidence in the academic literature for its existence. Additionally, the process of moral distress, moral residue, and the crescendo effect identified in this study provides evidence that discontent with the current model of providing care in the SICU environment emanates from the healthcare system itself, particularly provided the complaints related to administration/ management. 
The goal of future research is to focus on a unique aspect of human interaction discovered by conducting this study—talking about moral distress. The goal of this study was not to serve as therapy. However, one of the most interesting aspects of this study was the nurse participants' eagerness to discuss their experiences with moral distress. At the end of each of the interviews, nurse participants expressed that they had never talked about their experiences so candidly before. In particular, nurse participants noted that by communicating about their violations to their core values and professional duties they noted a feeling of clarity just from communicating their unresolved feelings, emotions, and stresses. Perhaps, this is because the researcher was well acquainted with nurses from both SICUs. Regardless, future research is warranted in this particular SICU environment and will be conducted, particularly related to conversations with nurses and addressing and acknowledging moral distress.

The goal of future research is to plan a longitudinal study that follows new graduate nurses from local schools of nursing and their inception in the SICU environment. The idea is to capture their perceptions and experiences from the moment they are hired in the SICU setting. They will be subsequently interviewed at several predetermined times and at several discrete time points, for example, during their hiring phase, six months after working on the unit, and then a year after working in the SICU. The goal will be two-fold: a) to demonstrate that an effective method of communicating with nurses about violations to their core values and duties may be the necessary groundwork needed to be helpful in resolving moral issues collaboratively and interprofessionally at the bedside and also subsequently empowering nurses to act in the name of quality patient care; and b) to explore the development of moral distress in new 
graduate nurses in order to better understand how to acknowledge and act on it — for example, what younger generations of nurses value and find necessary to provide quality patient care.

Future research will explore if there is a linear progression or perhaps some other mechanism of the development of moral distress and moral residue. The goal is to collaborate interprofessionally by working across disciplines and settings in order to promote buy-in from management that moral distress is a real phenomenon. This study demonstrated the need for a method that acknowledges moral distress through communication - and interviews provide a method to begin discussing violations to core values and duties in the SICU setting. Exploration of SICU nurses' perceptions and experiences in this study was accomplished through a qualitative descriptive approach and provides the groundwork for a future research trajectory that will seek to address the crescendo effect by developing interventions aimed at preventing unresolved moral distress and moral residue among SICU nurses through communication.

\section{Implications of Ignoring Moral Distress}

The major implication of ignoring moral distress completely disregards the moral issues that underpin the experience of SICU nurses. If nurses are experiencing and perceiving moral distress and emotional trauma at work in specific clinical situations in the SICU environment, then an investigation is warranted. For example, SICU nurses experienced moral distress providing care for particular types of patients (acute versus chronic conditions), age (young versus old), traumatic accident versus suicide or complex chronic healthcare conditions. By acknowledging these morally distressing experiences, ethical investigation into how and why they occurred will help to validate the experiences 
for the nurse, but also determine the underlying moral issue that enabled the experience of negativity and powerlessness by SICU nurses.

Importantly, there are implications for not addressing moral distress within a healthcare organization. Moral distress and moral residue affects the nurse mentally, physically, and emotionally - how it is managed or ignored at the unit/team and organization/system level is essential to understand. Moral residue is dependent upon unresolved, morally distressing events that linger, and as these events pile up, moral residue may manifest professionally in nurses as conscientious objection or withdrawal from the profession of nursing. It is unclear how many morally distressing events are needed to create a crescendo effect, because what is considered morally right and wrong may be different for individual nurses and informed by one's personal values, upbringing, and political, spiritual, and religious views. Furthermore, understanding how nurses manage the psychological stress of being complicit in immoral actions in the clinical environment is essential. If unhealthy coping mechanisms are employed to cope with moral distress, this will lead to unhealthy consequences and further burnout, withdrawal, turnover, and resignation from the nursing profession. Furthermore, even if nurses use healthy coping mechanisms performing their professional obligations and duties day-today, the clinical consequences remain, such as the unaddressed moral issue that initiated the experience of moral distress and the impact on the patient's quality of care. Thus, advocating for a healthy ethical workplace climate where these moral issues are addressed before they spiral out of control is critical. To that end, exploring the root causes of moral distress for critical care nurses in the SICU and how they are addressed is essential. 
If moral distress goes unaddressed, this could be due to several hypothesized reasons: (1) the nurse may not want to "rock the boat" and feels that it is just the nature of the healthcare culture; (2) feelings of moral distress may not occur in the nurse if the nurse believes that it is "just a job" that has no moral component; (3) the nurse is morally distraught, but does not have the education, language, self-assuredness, or self-confidence to speak-up, because the nurse feels that it is neither their duty or that their opinion does not matter - that is, the nurse may not know how to professionally manage the immoral wrongs in practice because they are not supported by management; and (4) the nurse is so burned out and jaded by clinical practice that the nurse intends to leave nursing; thus, addressing moral distress is the least of their concerns - the nurse either conscientiously objects or quits nursing to protect against further moral distress and harm. The significance of this study cannot be understated, because all coping must be viewed as a response to dealing with moral issues that occur in practice. In this way, all coping is protective coping — even though it may be harmful or maladaptive, because it is assumed that SICU nurses are coping just to handle the stressful nature of their practice.

Research questions to be addressed include the following: How do nurses in particular cope with the psychological stress of knowing they are complicit in moral wrongs at the bedside? How does moral distress impact nurses' home and professional life? Do they go home and drink alcohol, go back to school, do drugs, exercise, spend time with their family, play video games, work overtime to pay for things to allow them to forget, do they quit that job and go to another nursing job in another department, or do they simply leave nursing altogether? Why are the ethical dimensions of practice being ignored? Why don't ICUs have a standing policy on addressing ethical concerns at the 
bedside? Is a nightshift nurse able to consult an ethical consultant or ethics committee during the shift when a moral wrong is occurring? Are de-briefings offered as part of the general flow of unit activities, such as unit-based council meetings that employ outlets for ethical discussions?

It is important to understand these individual coping mechanisms for stress management, because healthcare units/teams and organizations/systems need to acknowledge, identify, and address experiences of moral distress and moral residue. This will enable practice that is ethical in nature by always considering what is in the patient's best interests, how to advocate for their quality of life, and integrate those concerns into an ethical/clinical model of healthcare delivery. This action will provide nurses with the resilience not only to address ethical problems in the workplace, but also cope with morally distressing issues, because clinical practice requires caring for people in dire circumstances. However, if nurses are able to provide quality care, regardless of the outcome, in a way that allows them to have their moral and ethical concerns about the dimensions of practice addressed so as to advocate for their patients, then the patients' best interests and quality of life will be assured. In this way, nursing practice and the quality of care will be just, equitable, and beneficent. 


\section{REFERENCES}

Ameri, M., Safavibayatneed, Z., \& Kavousi, A. (2016). Moral distress of oncology nurses and morally distressing situations in oncology units. Australian Journal Of Advanced Nursing, 33(3), 6-12.

American Association of Critical-Care Nurses (AACN). (2008). Public policy position statement: Moral distress [Online PDF]. Retrieved on 2016, November 11, from https://www.aacn.org/ /media/aacn-website/nursing-excellence/healthy-workenvironment/moral_distress.pdf?la=en

American Association of Critical-Care Nurses (AACN). (n.d.). The 4A's to rise above moral distress [Online PDF]. Retrieved on 2018, July, 28, from https://www.emergingrnleader.com/wpcontent/uploads/2012/06/4As_to_Rise_Above_Moral_Distress.pdf

American Nurses Association (ANA). (2015). Code of ethics for nurses with interpretative statements [Online PDF]. Retrieved on 2017, March 31 from http://nursingworld.org/DocumentVault/Ethics-1/Code-of-Ethics-for-Nurses.html

Angus, D. C., Barnato, A. E., Linde-Zwirble, W. T., Weissfeld, L. A., Watson, S. R., Rickert, T., Rubenfeld, G. D. (2004). Use of intensive care at the end of life in the United States: An epidemiologic study. Critical Care Medicine, 32(3), 638-643.

Austin, W., Lemermeyer, G., Goldberg, L., Bergum, V., \& Johnson, M. S. (2005). Moral distress in healthcare practice: The situation of nurses. HEC Forum, 17(1), 33-48. 
Beauchamp, T. L., \& Childress, J. F. (2009). Principles of biomedical ethics $\left(7^{\text {th }}\right.$ ed.). New York, New York: Oxford University Press.

Berlinger, N. (2016). When policy produces moral distress: Reclaiming conscience. Hastings Center Report, 46(2), 32-34. doi:10.1002/hast.547

Browning, A. M. (2013). Moral distress and psychological empowerment in critical care nurses caring for adults at end of life. American Journal of Critical Care, 22(2), 143-152. doi: http://dx.doi.org/10.4037/ajcc2013437

Bruce, C. R., Miller, S. M., \& Zimmerman, J. L. (2015). A qualitative study exploring moral distress in the ICU team: The importance of unit functionality and intrateam dynamics. Critical Care Medicine, 43(4), 823-831. doi:10.1097/CCM.0000000000000822

Campbell, S. M., Ulrich, C. M., \& Grady, C. (2016). A broader understanding of moral distress. American Journal Of Bioethics, 16(12), 2-9. doi:10.1080/15265161.2016.1239782

Carse, A. L. (1991). The voice of care: Implications for bioethical education. The Journal of Medicine and Philosophy, 16, 5-28.

Cherniss, C. (1980). Professional burnout in human service organizations. New York, NY: Praeger.

Choe, K., Kang, Y., \& Park, Y. (2015). Moral distress in critical care nurses: a phenomenological study. Journal of Advanced Nursing, 71(7), 1684-1693. doi:10.1111/jan.12638

Corley, M. (2002). Nurse moral distress: A proposed theory and research agenda. Nursing Ethics, 9(6), 636-650. doi:10.1191/0969733002ne557oa 
Corley, M., Elswick, R., Gorman, M., \& Clor, T. (2001). Development and evaluation of a moral distress scale. Journal of Advanced Nursing, 33(2), 250-256. doi: j.13652648.2001.01658.x10.1046/j.1365-2648.2001.01658.x

Corley, M. C. (1995). Moral distress of critical care nurses. American Journal of Critical Care, 4(4), 280-285.

Dartmouth Atlas of Health Care. (2018). End-of-life care [Website]. Retrieved on 2018, February 6, from http://www.dartmouthatlas.org/keyissues/issue.aspx?con=2944 Dartmouth Atlas of Health Care. (2018). Percent of deaths associated with ICU admission [Website]. Retrieved on 2018, February 6, from http://www.dartmouthatlas.org/data/table.aspx?ind=14

Davion, V. (2006). Health care in the United States: Evil intentions and collective responsibility. Midwest Studies in Philosophy, 30(1), 325-327.doi: $10.1111 / \mathrm{j} .1475-4975.2006 .00143 . \mathrm{x}$

Davis, S., Schrader, V., \& Belcheir, M. J. (2012). Influencers of ethical beliefs and the impact on moral distress and conscientious objection. Nursing Ethics, 19(6), 738749. doi: $10.1177 / 0969733011423409$

DeKeyser, Ganz, F., \& Berkovitz, K. (2012). Surgical nurses' perceptions of ethical dilemmas, moral distress and quality of care. Journal Of Advanced Nursing, 68(7), 1516-1525. doi:10.1111/j.1365-2648.2011.05897.x

Dudzinski, D. M. (2016). Navigating moral distress using the moral distress map. Journal of Medical Ethics, 42(5), 321-324. doi: 10.1136/medethics-2015-103156 
Earle, V. (2010). Phenomenology as research method or substantive metaphysics? An overview of phenomenology's uses in nursing. Nursing Philosophy, 11(4), 286296. doi: 10.1111/j.1466-769X.2010.00458.x

Epstein, E. (2007, January). End-of-life experiences of parents, nurses and physicians in the newborn intensive care unit. Retrieved from ProQuest Digital Dissertations. (AAT 3286595)

Epstein, E. (2008). End-of-life experiences of nurses and physicians in the newborn intensive care unit. Journal of Perinatology, 28(11), 771-778. doi:10.1038/jp.2008.96

Epstein, E., \& Hamric, A. (2009). Moral distress, moral residue, and the crescendo effect. Journal Of Clinical Ethics, 20(4), 330-342.

Epstein, E. G., Hurst, A. R., Mahanes, D., Marshall, M. F., \& Hamric, A. B. (2016). Is broader better?. American Journal Of Bioethics, 16(12), 15-17. doi:10.1080/15265161.2016.1242669

Epstein, E. G., \& Hurst, A. R. (2017). Looking at the positive side of moral distress: Why it's a problem. The Journal of Clinical Ethics, 28(1), 37-41.

Flannery, L., Ramjan, L. M., \& Peters, K. (2016). End-of-life decisions in the intensive care unit (ICU) - Exploring the experiences of ICU nurses and doctors - A critical literature review. Australian Critical Care, 29(2), 97-103. doi:10.1016/j.aucc.2015.07.004

Fleck, L. M. (2009). Just caring. New York: Oxford University Press.

Glaser, B. G. (1978). Theoretical sensitivity: Advances in the methodology of grounded theory. Mill Valley, California: The Sociology Press. 
Gutierrez, K. M. (2005). Critical care nurses' perceptions of and responses to moral distress. Dimensions of Critical Care Nursing, 24(5), 229-241.

Hamric, A. B. (2014). A case study of moral distress. Journal of Hospice \& Palliative Nursing, 16(8), 457-463. doi: 10.1097/NJH.0000000000000104

Hamric, A. B. (2012). Empirical research on moral distress: Issues, challenges, and opportunities. HEC Forum, 24(1), 39-49. doi: https://doiorg.echo.louisville.edu/10.1007/s10730-012-9177-x

Hamric, A. B., \& Blackhall, L. (2007). Nurse-physician perspectives on the care of dying patients in intensive care units: collaboration, moral distress, and ethical climate. Critical Care Medicine, 35(2), 422-429.

Hamric, A. B., Borchers, C. T., \& Epstein, E. G. (2012). Development and testing of an instrument to measure moral distress in healthcare professionals. AJOB Primary Research, 3(2), 1-9. doi: 10.1080/21507716.2011.652337

Hamric, A. B., \& Epstein, E. G. (2017). A health system-wide moral distress consultation service: Development and Evaluation. HEC Forum, 29(2), 127-143. doi: $10.1007 / \mathrm{s} 10730-016-9315-\mathrm{y}$

Hamric, A. B., \& Wocial, L. D. (2016). Institutional ethics resources: Creating moral spaces. Nurses at the Table: Nursing, Ethics, and Health Policy, special report, Hastings Center Report 46(5), S22-S27. doi: 10.1002/hast.627

Hanna, D. R. (2004). Moral distress: The state of the science. Research and Theory for Nursing Practice, 18(1), 73-93.

Henrich, N. J., Dodek, P. M., Gladstone, E., Alden, L., Keenan, S. P., Reynolds, S., \& Rodney, P. (2017). Consequences of moral distress in the intensive care unit: A 
qualitative study. American Journal Of Critical Care, 26(4), e48-e57. doi:10.4037/ajcc2017786

Hiler, C. A. (2018). Predictors of moral distress in a US sample of critical care nurses. American Journal Of Critical Care, 27(1), 59-66. doi:10.4037/ajcc2018968

Huynh, T. N., Kleerup, E. C., Raj, P. P., \& Wenger, N. S. (2014). The opportunity cost of futile treatment in the ICU*. Critical Care Medicine, 42(9), 1977-1982. doi:10.1097/CCM.0000000000000402

Huynh, T. N., Kleerup, E. C., Wiley, J. F., Savitsky, T. D., Guse, D., Garber, B. J., \& Wenger, N. S. (2013). The frequency and cost of treatment perceived to be futile in critical care. JAMA Internal Medicine, 173(20), 1887-1894 8p. doi:10.1001/jamainternmed.2013.10261

Institute of Medicine (IOM). (2011). The future of nursing: Leading change, advancing health. Washington, D.C.: National Academies Press.

International Council of Nurses (ICN). (2012). The ICN code of ethics for nurses [Online PDF]. Retrieved on 2016, October 7, from http://www.icn.ch/images/stories/documents/about/icncode_english.pdf

Jameton, A. (1984). Nursing practice: The ethical issues. Englewood Cliffs, NJ: PrenticeHall.

Jameton, A. (1993). Dilemmas of moral distress: Moral responsibility and nursing practice. AWHONN's Clinical Issues in Perinatal \& Women's Health Nursing, $4(4), 542-551$. 
Johnson-Coyle, L., Opgenorth, D., Bellows, M., Dhaliwal, J., Richardson-Carr, S., \& Bagshaw, S. M. (2016). Moral distress and burnout among cardiovascular surgery intensive care unit healthcare professionals: A prospective cross-sectional survey. Canadian Journal Of Critical Care Nursing, 27(4), 27-36.

Jonsen, A. R. (1991). Of balloons and bicycles; or, the relationship between ethical theory and practical judgment. The Hastings Center Report, 21(5), 14-16. doi: $10.2307 / 3562885$

Kälvemark, S., Höglund, A. T., Hansson, M. G., Westerholm, P., \& Arnetz, B. (2004). Living with conflicts-ethical dilemmas and moral distress in the health care system. Social Science Medicine, 58(6), 1075-1084.

Kantymir, L., \& McLeod, C. (2014). Justification for conscience exemptions in health care. Bioethics, 28(1), 16-23. doi: 10.1111/bioe.12055

Krippendorff, K. (2013). Content analysis: An introduction to its methodology ( $\left.3^{\text {rd }} \mathrm{ed}.\right)$. Thousand Oaks, California: SAGE Publications, Inc.

Lachman, V. D. (2016). Ethics, law, and policy. Moral resilience: Managing and preventing moral distress and moral residue. MEDSURG Nursing, 25(2), 121-124.

Lazarus, R. S., \& Folkman, S. (1984). Stress, appraisal and coping. New York, NY: Springer.

Leiter, M. P., \& Harvie, P. (1998). The correspondence of patient satisfaction and nurse burnout. Social Science \& Medicine, 47(10), 1611-1617.

Lievrouw, A., Vanheule, S., Deveugele, M., De Vos, M., Pattyn, P., Belle, V., \& Benoit, D. D. (2016). Coping with moral distress in oncology practice: Nurse and 
physician strategies. Oncology Nursing Forum, 43(4), 505-512.

doi:10.1188/16.ONF.505-512

Lincoln, Y. S., \& Guba, E. G. (1985). Establishing trustworthiness. In Y. S. Lincoln \& E. G. Guba, Naturalistic inquiry. Beverly Hills, CA: Sage Publications. pp. 289-331.

Lützén, K., Cronqvist, A., Magnusson, A., \& Andersson, L. (2003). Moral stress:

synthesis of a concept. Nursing Ethics, 10(3), 312-322.

doi:10.1191/0969733003ne608oa

Manara, D. F., Villa, G., \& Moranda, D. (2014). In search of salience: Phenomenological analysis of moral distress. Nursing Philosophy, 15(3), 171-182. doi:10.1111/nup.12048

Mason, D., Gardner, D., Outlaw, F. H. \& O'Grady, E. (2016). Policy and politics in nursing and health care ( $7^{\text {th }}$ ed.). St. Louis, MO: Saunders.

McAndrew, N. S., Leske, J. S., \& Garcia, A. (2011). Influence of moral distress on the professional practice environment during prognostic conflict in critical care. Journal Of Trauma Nursing, 18(4), 221-230. doi:10.1097/JTN.0b013e31823a4a12

McCarthy, J., \& Deady, R. (2008). Moral distress reconsidered. Nursing Ethics, 15(2), 254-262. doi: 10.1177/0969733007086023

McCarthy, J., \& Gastmans, C. (2015). Moral distress: A review of the argument-based nursing ethics literature. Nursing Ethics, 22(1), 131-152. doi: http://dx.doi.org.echo.louisville.edu/10.1177/0969733014557139 
Miles, M. B., Huberman, A. M., \& Saldaña, J. (2014). Qualitative data analysis: A methods sourcebook ( $3^{\text {rd }}$ ed.). Thousand Oaks, California: SAGE Publications, Inc.

Mitton, C., Peacock, S., Storch, J., Smith, N., \& Cornelissen, E. (2010). Moral distress among healthcare managers: Conditions, consequences and potential responses. Healthcare Policy, 6(2), 99-112.

Mobley, M., Rady, M., Verheijde, J., Patel, B., \& Larson, J. (2007). The relationship between moral distress and perception of futile care in the critical care unit. Intensive \& Critical Care Nursing, 23(5), 256-263.

Munhall, P. L. (2012). Nursing research: A qualitative perspective (5 $5^{\text {th }}$ ed.). Sudbury, Massachusetts: Jones and Bartlett Publishers.

Neville, T. H., Wiley, J. F., Yamamoto, M. C., Flitcraft, M., Anderson, B., Curtis, J. R., \& Wenger, N. S. (2015). Concordance of nurses and physicians on whether critical care patients are receiving futile treatment. American Journal Of Critical Care, 24(5), 403-411. doi:10.4037/ajcc2015476

Niederman, M., \& Berger, J. (2010). The delivery of futile care is harmful to other patients. Critical Care Medicine, 38, S518-S522. doi:10.1097/CCM.0b013e3181f1cba5

O’Connell, C. B. (2015). Gender and the experience of moral distress in critical care nurses. Nursing Ethics, 22(1), 32-42. doi:10.1177/0969733013513216

Oh, Y., \& Gastmans, C. (2015). Moral distress experienced by nurses: A quantitative literature review. Nursing Ethics, 22(1), 15-31. doi:10.1177/0969733013502803 
Olson, L. (1998). Hospital nurses' perceptions of the ethical climate of their work setting. Image: Journal Of Nursing Scholarship, 30(4), 345-349.

Patton, M. Q. (2015). Qualitative research \& evaluation methods (4 ${ }^{\text {th }}$ ed.). Thousand Oaks, California: SAGE Publications.

Pauly, B., Varcoe, C., Storch, J., \& Newton, L. (2009). Registered nurses' perceptions of moral distress and ethical climate. Nursing Ethics, 16(5), 561-573. doi:10.1177/0969733009106649

Piers, R. D., Van den Eynde, M., Steeman, E., Vlerick, P., Benoit, D. D., \& Van Den Noortgate, N.J. (2012). End-of-life care of the geriatric patient and nurses' moral distress. Journal Of The American Medical Directors Association, 13(1), 80.e780.e13. doi:10.1016/j.jamda.2010.12.014

Reddy, D. S., O'Horo, J. C., Hoskote, S. S., \& Moua, T. (2015). Judgment of futile care in the ICU. Critical Care Medicine, 43(5), e151-e151 1p. doi:10.1097/CCM.0000000000000874

Richards, L., \& Morse, J.M. (2013). Read me first for a user's guide to qualitative methods $\left(3^{\text {rd }}\right.$ ed.). Thousand Oaks CA: Sage Publications.

Robinson, E. M., Lee, S. M., Zollfrank, A., Jurchak, M., Frost, D., \& Grace, P. (2014). Enhancing moral agency: Clinical ethics residency for nurses. Hastings Center Report, 44(5), 12-20. doi:10.1002/hast.353

Robinson, R. (2010). Registered nurses and moral distress. Dimensions Of Critical Care Nursing, 29(5), 197-202. doi:10.1097/DCC.0b013e3181e6c344 
Robinson, R., \& Stinson, C. K. (2016). Moral Distress: A qualitative study of emergency nurses. Dimensions Of Critical Care Nursing, 35(4), 235-240. doi:10.1097/DCC.0000000000000185

Rochefort, C., \& Clarke, S. (2010). Nurses' work environments, care rationing, job outcomes, and quality of care on neonatal units. Journal Of Advanced Nursing, 66(10), 2213-2224. doi:10.1111/j.1365-2648.2010.05376.x

Rodgers, B. (2005). Developing nursing knowledge: Philosophical traditions and influence. New York: Lippincott Williams \& Wilkins.

Rodgers, B. L., \& Cowles, K. V. (1993). The qualitative research audit trail: A complex collection of documentation. Research in Nursing \& Health, 16(3), 219-226.

Rushton, C. H. (2016). Ethics in critical care. Moral resilience: A capacity for navigating moral distress in critical care. AACN Advanced Critical Care, 27(1), 111-119. doi:10.4037/aacnacc2016275

Rushton, C. H., Batcheller, J., Schroeder, K., \& Donohue, P. (2015). Burnout and resilience among nurses practicing in high-intensity settings. American Journal of Critical Care, 24(5), 412-421. doi:10.4037/ajcc2015291

Rushton, C. H., Kaszniak, A. W., \& Halifax, J. S. (2013). A framework for understanding moral distress among palliative care clinicians. Journal Of Palliative Medicine, 16(9), 1074-1079. doi:10.1089/jpm.2012.0490

Russell, A. C. (2012). Moral distress in neuroscience nursing: An evolutionary concept analysis. Journal of Neuroscience Nursing, 44(1), 15-26. doi: http://dx.doi.org/10.1097/JNN.0b013e31823ae4cb 
Sabin, J. E. (2017). Using moral distress for organizational improvement. The Journal of Clinical Ethics, 28(1), 33-36.

Sandelowski, M. (1986). The problem of rigor in qualitative research. ANS. Advances in Nursing Science, 8(3), 27-37.

Sandelowski, M. (1995). Focus on qualitative methods. Sample size in qualitative research. Research in Nursing \& Health, 18(2), 179-183. doi: 10.1002/nur.4770180211

Sandelowski, M. (2000). Focus on research methods. Whatever happened to qualitative description?. Research in Nursing and Health, 23(4), 334-340.

Sandelowski, M. (2010). What's in a name? Qualitative description revisited. Research in Nursing \& Health, 33(1), 77-84. doi: 10.1002/nur.20362

Sauerland, J., Marotta, K., Peinemann, M. A., Berndt, A., \& Robichaux, C. (2014). Assessing and addressing moral distress and ethical climate, part 1. Dimensions Of Critical Care Nursing, 33(4), 234-245. doi:10.1097/DCC.0000000000000050

Savel, R. H., \& Munro, C. L. (2015). Moral distress, moral courage. American Journal of Critical Care, 24(4), 276-278. doi: http://dx.doi.org.echo.louisville.edu/10.4037/ajcc2015738

Schneiderman, L. J., Jecker, N. S., \& Jonsen, A. R. (1990). Medical futility: Its meaning and ethical implications. Annals of Internal Medicine, 112(12): 949-954.

Schweickert, W. D., Pohlman, M. C., Pohlman, A. S., Nigos, C., Pawlik, A. J., Esbrook, C. L., Spears, L., Miller, M., Franczyk, M., Deprizio, D., Schmidt, G. A., Bowman, A., Barr, R., McCallister, K. E., Hall, J. B., \& Kress, J. P. (2009). Early physical and occupational therapy in mechanically ventilated, critically ill 
patients: A randomised controlled trial. Lancet, 373(9678), 1874-1882. doi:

10.1016/S0140-6736(09)60658-9

Shoorideh, F. A., Ashktorab, T., Yaghmaei, F., \& Alavi Majd, H. (2015). Relationship between ICU nurses' moral distress with burnout and anticipated turnover. Nursing Ethics, 22(1), 64-76. doi:10.1177/0969733014534874

Solomon, M. Z., O’Donnell, L., Jennings, B., Guilfoy, V., Wolf, S. M., Nolan, K., . . . Donnelley, S. (1993). Decisions near the end of life: Professional views on lifesustaining treatments. American Journal of Public Health, 83(1), 14-23. doi: http://dx.doi.org/10.2105/AJPH.83.1.14

Spenceley, S., Witcher, C. G., Hagen, B., Hall, B., \& Kardolus-Wilson, A. (2017). Sources of moral distress for nursing staff providing care to residents with dementia. Dementia (14713012), 16(7), 815-834.

doi: $10.1177 / 1471301215618108$

St Ledger, U., Begley, A., Reid, J., Prior, L., McAuley, D., \& Blackwood, B. (2013). Moral distress in end-of-life care in the intensive care unit. Journal Of Advanced Nursing, 69(8), 1869-1880. doi:10.1111/jan.12053

Taylor-Ford, R. L. (2013). Moral distress in end-of-life care: Promoting ethical standards of executive nursing practice. Nurse Leader, 11(3), 51-54. doi:10.1016/j.mnl.2013.01.005

Thomas, T. A., \& McCullough, L. B. (2015). A philosophical taxonomy of ethically significant moral distress. The Journal of Medicine and Philosophy, 40(1), 102120. doi: 0.1093/jmp/jhu048 
Thomas, T. A., \& McCullough, L. B. (2017). Focus more on causes and less on symptoms of moral distress. The Journal of Clinical Ethics, 28(1), 30-32.

Vahey, D. C., Aiken, L. H., Sloane, D. M., Clarke, S. P., \& Vargas, D. (2004). Nurse burnout and patient satisfaction. Med Care, 42(2), doi: 10.1097/01.mlr.0000109126.50398.5a

Varcoe, C., Pauly, B., Webster, G., \& Storch, J. (2012). Moral distress: Tensions as springboards for action. HEC Forum, 24(1), 51-62. doi: 10.1007/s10730-0129180-2

Waldrop, D. P., \& Meeker, M. A. (2012). Communication and advanced care planning in palliative and end-of-life care. Nursing Outlook, 60(6), 365-369. doi: 10.1016/j.outlook.2012.08.012

Weaver, K., Morse, J., \& Mitcham, C. (2008). Ethical sensitivity in professional practice: Concept analysis. Journal of Advanced Nursing, 62(5), 607-618.

Weber, E. (2016). Moral distress, workplace health, and intrinsic harm. Bioethics, 30(4), 244-250. doi:10.1111/bioe.12181

Webster G. C, \& Baylis F. E. (2000). Moral residue. In S. B. Rubin \& L. Zoloth (Eds.), Margin of error: The ethics of mistakes in the practice of medicine (217-230). Haggerstown: University Publishing Group.

Whitehead, P. B., Herbertson, R. K., Hamric, A. B., Epstein, E. G., \& Fisher, J. M. (2015). Moral distress among healthcare professionals: Report of an institutionwide survey. Journal of Nursing Scholarship, 47(2), 117-125. doi:10.1111/jnu.12115 
Wilkinson, J. (1989). Moral distress: A labor and delivery nurse's experience. JOGNN: Journal Of Obstetric, Gynecologic \& Neonatal Nursing, 18(6), 513-519. doi:10.1111/j.1552-6909.1989.tb00503.x

Wilkinson, J. M. (1987). Moral distress in nursing practice: Experience and effect. Nursing Forum, 23(1), 16-29. doi: http://dx.doi.org.echo.louisville.edu/10.1111/j.1744-6198.1987.tb00794.x

Wilson, M. A., Goettemoeller, D. M., Bevan, N. A., \& McCord, J. M. (2013). Moral distress: Levels, coping and preferred interventions in critical care and transitional care nurses. Journal Of Clinical Nursing, 22(9/10), 1455-1466. doi:10.1111/jocn.12128

Woods, M., Rodgers, V., Towers, A., \& La Grow, S. (2015). Researching moral distress among New Zealand nurses: A national survey. Nursing Ethics, 22(1), 117-130. doi:10.1177/0969733014542679 


\section{APPENDIX A}

Model of the Moral Residue Crescendo Effect

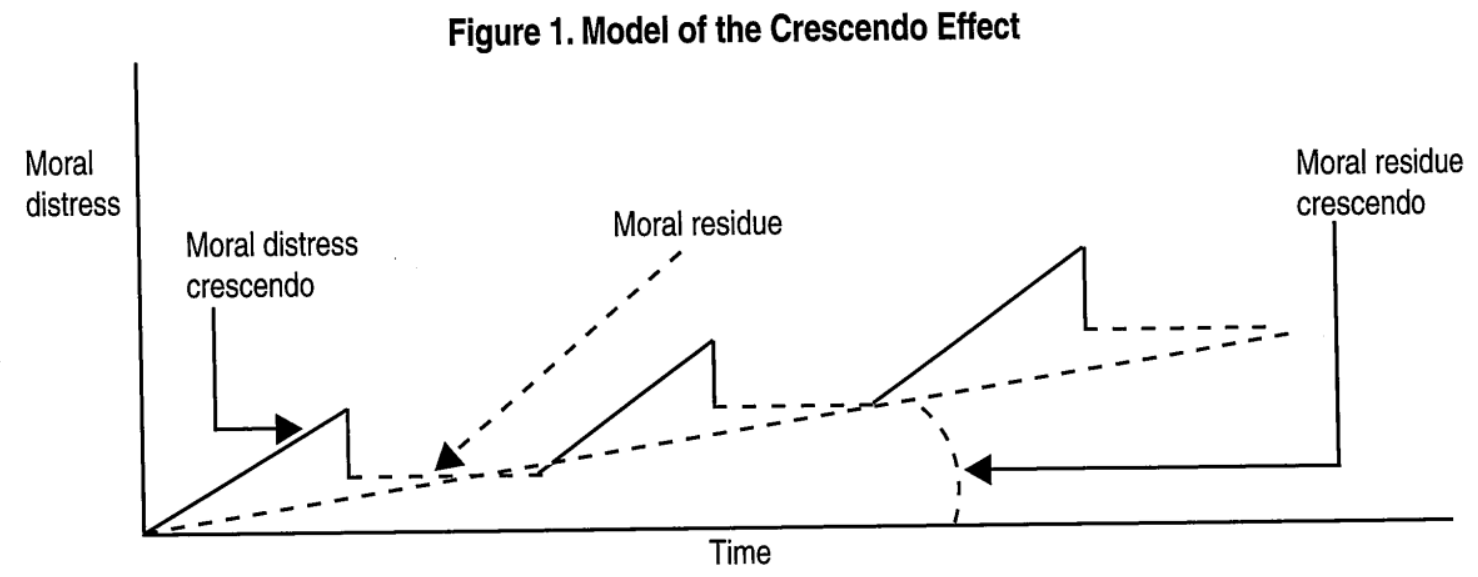

Solid lines indicate moral distress; dotted lines indicate moral residue.

Figure 1. Moral distress and moral residue crescendo effect. Reprinted from "Moral

Distress, Moral Residue, and the Crescendo Effect," by E. G. Epstein and A. B. Hamric, 2009, The Journal of Clinical Ethics, 20(4), p. 333. Copyright 2009 by The Journal of Clinical Ethics. 


\section{APPENDIX B}

\section{Demographic Questionnaire}

Please describe yourself by marking one answer for each of the following questions.

1. Age: (years)

How old are you:

2. Sex:

[ ] Female

[] Male

[] Transgender

3. Race

[ ] Caucasian

[ ] Black or African American

[] American Indian

[] Alaskan

[ ] Asian

[ ] Pacific Islander

4. Ethnicity

[ ] Hispanic

[ ] Non-Hispanic

5. Highest nursing degree completed:
[ ] Diploma
[] Associate
[] Bachelors
[ ] Masters
[ ] DNP
[] $\mathrm{PhD}$

6. Average number of hours worked per week on your primary nursing unit: hours 
7. Number of years you have been employed as an RN:

years months

8. Number of years employed in current specialty/ICU: years months

9. Who from the hospital do you socialize with?

a. Who from outside the hospital do you socialize with?

b. How do you relax after work? 


\section{APPENDIX C}

\section{Interview Guide}

Introduction: Hello, my name is Adam Booth and I have been an SICU nurse for the past nine years.

Currently I am a PhD candidate at the University of Louisville School of Nursing and am conducting my research study to explore ICU nurses' perceptions of patient care situations that might have been distressing to you. For example, you may feel like something is morally wrong and the patient is not getting the care they deserve or would want, that the patient is receiving care that may potentially harm him or her. Many times clinical situations are confusing and may conflict with your own views of what is morally right and wrong, or with the views of the patient and family, or with those of physicians and other healthcare team members.

I am interested in exploring your perceptions and experiences about these moral gray areas associated with clinical practice in the SICU and your views about why these issues become a problem, how these issues affect the quality of your patient care, and how you deal with these stresses.

Thank you for taking time out of your busy schedule to talk to me. I plan to interview 10 to 20 SICU nurses about their perceptions and experiences about these moral gray areas of clinical practice. Please be as honest as possible. Your responses will be anonymous and confidential and will not be shared with administration or management.

Verbatim audio recordings of the interviews will not have identifying information to maintain confidentiality. Confidentiality will be protected using de-identified notes, memos, and transcriptions throughout the research process. A Certificate of Confidentiality $(\mathrm{CoC})$ from

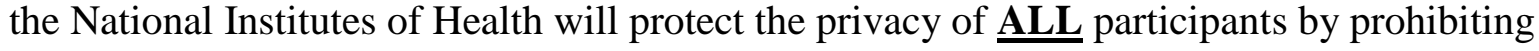
disclosure of identifiable, sensitive research information to anyone not connected to the research. The $\mathrm{CoC}$ will prevent any of the participants from being identified by hospital administration, clinical directors, clinical managers, charge nurses, and other co-workers.

\section{Questions:}

1. Tell me about a time in your nursing experience in the SICU when you perceived something was morally wrong: for example a patient was not getting care such that it might potentially harm the patient. Have you experienced a situation like this?

What about the clinical experiences made you perceive it as wrong? 
a. How did you know it was "wrong"?

b. Describe how this situation shaped your perceptions.

c. Describe what was stressful about this experience.

d. Describe how you dealt with these stresses at work.

e. Describe how you dealt with these stresses at home.

f. Describe how your experiences with the stressful situation effected your perceptions of the quality of your patient care?

a. How did that make you feel? [PROMPT]

b. What did you think about this? [PROMPT]

g. Did these experiences ever make you want to leave your job and/or nursing?

a. Tell me more about this. [PROMPT]

2. Describe any clinical experiences that you have experienced in the SICU in which you knew the care that needed to be provided or resources that were needed, but you could not provide either.

a. How did these situations make you feel?

b. Describe any situations that made you perceive frustration or powerlessness with caregiving in SICU.

c. Describe what was most intense and bothersome about these experiences in SICU.

d. How frequently do these situations happen in SICU?

3. Have you ever felt that your work in the SICU was unbearable?

a. How would you describe your experiences with these situations?

b. What makes coming to work harder?

4. Is there an accumulation of multiple stressful situations that cross some personal/professional red line for you?

a. How do you know you have crossed the red line?

b. What do you do if the red line is repeatedly crossed?

c. How are your values and personal moral integrity impacted?

d. How is your care impacted?

5. How did you cope with these clinical experiences? 
a. Who do you talk to about your clinical experiences?

b. Use of team meetings? [PROMPT]

c. Ethics committee? [PROMPT]

d. Policy change? [PROMPT]

e. Any other resources that may have been used to resolve the experience? [PROMPT]

6. Are there any other comments you want to share?

Thank you for sharing your experiences with me.

Your perspectives will be helpful in designing future interventions to help other SICU nurses. 


\title{
CURRICULUM VITA
}

\author{
Adam T. Booth, PhD, BSN, RN \\ Indiana University Southeast School of \\ Nursing \\ Indiana University Southeast \\ Telephone: (502) 439-1861
}

Email: bootha@iu.edu

\section{Education}

2014-2020

University of Louisville, Louisville,

Kentucky

2015-2016

University of Louisville, Louisville,

Kentucky

(Health Professions

Education)

2010

Indiana University Southeast, New

Albany, Indiana

BSN (Nursing)

\section{Licenses and Certifications}

2010 - Present

Registered Nurse, Kentucky

1124164

2010 - Present

Advanced Cardiac Life Support and Basic

Life Support Certifications

\section{Professional Positions}

2018-Present

August 2014-July 2018
Visiting Assistant Nursing Professor

Graduate Research Assistant
School of Nursing, Indiana University Southeast, New

Albany, Indiana School of Nursing, University of Louisville, Louisville, Kentucky 
SICU, University of Louisville Hospital,

2013-2014

Staff Nurse Louisville, Kentucky Pre- and Post Operative Care, Norton Healthcare Pavilion, Louisville, Kentucky

\section{Scholarship: Funding}

2019 Mosaic Faculty Fellow

Role and effort: A one-year commitment to transforming teaching and contributing to the development of Indiana University's learning spaces Funding agency: Indiana University

Total costs: $\$ 1,000$

Funding period: September 2019 - Spring 2020

2018 Fall Online Teaching Cohort by the Institute for Learning and Teaching Excellence (ILTE)

Role and effort: Development of a Foundations of Bioethics course with an ILTE consultant

Funding agency: Indiana University Southeast

Total costs: $\$ 1,000$

Funding period: Fall 2018 semester

Simulation Immersion and Mastery (SIM) Institute

Role and effort: Attended four-day intensive after receiving funding

Funding agency: Indiana University Southeast

Total costs: $\$ 2,000$

Funding period: 8/6/2018 - 8/9/2018

Changes in End-of-Life Education for Critical Care Healthcare

Professionals

Role and effort: Presenter

Funding agency: Ruth Craddock Award

Total costs: $\$ 600$

Funding period: 2/24/2016 - 2/28/2016

Changes in End-of-Life Education for Critical Care Healthcare

Professionals

Role and effort: Presenter

Funding agency: Graduate Student Council

Total costs: $\$ 350$

Funding period: $2 / 24 / 2016-2 / 28 / 2016$ 
PhD Jonas Nurse Leader Scholar

Role and effort: Recipient of the Jonas Nurse Scholar Program

Scholarship

Funding agency: Jonas Center for Nursing and Veterans Healthcare and

American Association of Colleges of Nursing

Total costs: $\$ 20,000$

Funding period: Fall 2016 - Spring 2018

Nurses' Experiences Caring for Patients at the End-of-Life in the ICU

Role and effort: Presenter

Funding agency: School of Nursing - Nursing Graduate Student Award

Research!Louisville 2016

Total costs: $\$ 400$

Funding period: October 12, 2016

\section{Scholarship: Publications}

Booth, A.T., \& Lehna, C. (2016). Advanced directives and advanced care planning for healthcare professionals. Kentucky Nurse, 64(2), 7-10.

\section{Scholarship: Presentations}

\section{Local, State, and Regional Presentations (as APA citations, in chronological order)}

Booth, A., \& Lehna, C. (2015, October 27). Critical care healthcare professionals provide direction for end-of-life education. Norton Healthcare's Research!Louisville Nursing Symposium and Virtual Poster Fair. Louisville, KY.

Booth, A., \& Lehna, C. (2015, October 28). Critical care healthcare professionals provide direction for end-of-life education. Kosair Charities Clinical and Translational Research Building's Research!Louisville Poster Session Research Showcase. Louisville, KY.

Booth, A., \& Lehna, C. (2016, February 26). Changes in end-of-life education for critical care healthcare professionals. Southern Nursing Research Society. Williamsburg, VA.

Booth, A. (2016, April 1). Moral distress. Graduate Student Regional Research Conference Three-Minute Thesis Competition. Louisville, KY.

Booth, A., \& Lehna, C. (2016, April 1). Changes in end-of-life education for critical care healthcare professionals. Graduate Student Regional Research 
Conference Community Engagement Symposium Paper Presentation. Louisville, KY.

Booth, A., \& Christian, B.J. (2016, April 2). Moral distress: A Concept analysis. Graduate Student Regional Research Conference Poster Session. Louisville, KY.

Booth, A., \& Lehna, C. (2016, April 2). Changes in end-of-life education for critical care healthcare professionals. Graduate Student Regional Research Conference Podium Paper Session. Louisville, KY.

Booth, A., \& Christian, B.J. (2016, October 7). Moral distress: A Concept analysis. American Society for Bioethics and Humanities. Washington, D.C.

Booth, A., \& Shawler, C. (2016, October 10). Nurses' experiences caring for patients at the end-of-life in the ICU. Baptist Health Louisville's

Research!Louisville: Supporting Nursing Practice Through Research, Evidence-Based Practice and Quality Improvement. Louisville, KY.

Booth, A., \& Shawler, C. (2016, October 12). Nurses' experiences caring for patients at the end-of-life in the ICU. Kosair Charities Clinical and Translational Research Building's Research!Louisville Poster Session Research Showcase. Louisville, KY.

Booth, A., \& Shawler, C. (2017, February 25). Nurses' experiences caring for patients at the end-of-life in the ICU. Southern Nursing Research Society. Dallas, TX.

Booth, A., \& Kolers, A. (2017, September 13). Moral distress: An indicator of immoral practice. Kosair Charities Clinical and Translational Research Building's Research!Louisville Poster Session Research Showcase. Louisville, KY.

\section{Scholarship Activities}

April 8, 2015

November 16, 2015

March 7, 2016
Guest Speaker at Undergraduate Research Scholar Program Meeting, University of Louisville, Louisville, Kentucky Guest Speaker at University of Louisville School of Nursing PhD/DNP Information Session, University of Louisville, Louisville, Kentucky Presentation of "Changes in End-of-Life Education for Critical Care Healthcare Professionals" research findings at University of Louisville School of 
Nursing Research Assembly, University of Louisville, Louisville, Kentucky

April 11, 2016

Guest Speaker at Undergraduate Research Scholar Program Meeting, University of Louisville, Louisville, Kentucky

October 18, 2016

Guest Speaker at University of Louisville School of Nursing PhD/DNP Information Session, University of Louisville, Louisville, Kentucky

January 2015 - Current

Editor-in-Chief of University of Louisville School of Nursing Newsletter, University of Louisville, Louisville,

October 2015 - Current Kentucky

Research and Scholarship Committee

Graduate Student Representative for the University of Louisville School of

February 26, 2018 Nursing, University of Louisville, Louisville, Kentucky

Guest Speaker at the University of Louisville Spring Research Assembly: Discussed Moral Distress Research

February 27, 2018

March 6, 2018

July $23-27,2018$

Interest and Jonas Nurse Leader Scholarship, University of Louisville, Louisville, Kentucky

Guest Lecturer for a course in the Bioethics and Medical Humanities Department at the University of Louisville from 4:00p.m. to 6:00p.m.: Current Controversies in Bioethics-Discussed Moral Distress Research Interest, University of Louisville, Louisville, Kentucky

Guest Speaker at University of Louisville School of Nursing PhD/DNP Information Session, University of Louisville, Louisville, Kentucky 
Accepted to participate in Summer 2018

STEM Graduate Teaching Assistant

Academy, sponsored by the School of

Interdisciplinary and Graduate Studies

and led by University of Louisville faculty

and staff. Discussed the following:

Introduction to STEM Pedagogy and

Inclusive Teaching; Active Learning in

STEM Disciplines; Science of Learning;

Teaching Methods in STEM Disciplines;

and Micro-Teaching for and Providing

Feedback to your Peers. University of

Louisville, Louisville, Kentucky

\section{Teaching: Publications}

Booth, A.T., \& Lehna, C. (2016). Advanced directives and advanced care planning for healthcare professionals. Kentucky Nurse, 64(2), 7-10.

\section{Service: Committees}

Indiana University Southeast Budgetary Affairs Committee Fall 2019

Indiana University Southeast Faculty Senate

Fall 2019

Indiana University Southeast Learning Spaces Committee

Summer 2019

Baptist Floyd Evidence Based Practice Steering Committee Fall 2018

Indiana University Southeast Learning Spaces Committee Summer 2019

American Association of Critical-Care Nurses 12706185

American Nurses Association 03134463

Kentucky Nurses Association

03134463

American Society for Bioethics and Humanities 00095803 
National League for Nursing 576127 